\title{
Fracture system characterisation and implications for fluid flow in volcanic and metamorphic rocks
}

\author{
By Cécile Massiot
}

\author{
A thesis \\ submitted to Victoria University of Wellington \\ in fulfilment of the requirements for the degree of \\ Doctor of Philosophy in Geophysics.
}

Victoria University Wellington

2017 

"Reality is frequently inaccurate."

Douglas Adams

"L'existence d'une grammaire anglaise est une question sur laquelle il est permis d'émettre des doutes"

Salvador De Madariaga 



\section{Abstract}

Fluid flow pathways in volcanic and metamorphic rocks are dominantly controlled by fracture systems. Although fracture systems are critical for developing reservoirs in an economical and sustainable way, and for understanding processes that cause earthquakes, they are often poorly constrained. This thesis studies the geometry of fracture systems, the factors influencing their geometries, and their possible impacts on permeability in three contrasting settings: an outcropping andesite lava flow of the Ruapehu volcano; the andesite-hosted Rotokawa geothermal reservoir; and the Alpine Fault hangingwall metamorphosed schists. We use datasets from a combination of cores, acoustic borehole televiewer (BHTV) logs, outcrop scanlines, and terrestrial laser scanner (TLS) point clouds, which span multiple scales of observation.

Fracture geometries are studied in a young ( $\sim 6$ ka-old) blocky andesitic lava flow on Ruapehu volcano, as a representative example of weakly-altered andesitic lava flows emplaced over gentle topography in the absence of glaciers. Fractures were formed during cooling and emplacement of the lava flow. Fractures are automatically detected from the 3-D TLS point cloud of an outcrop area of $\sim 3090 \mathrm{~m}^{2}$ using a plane detection algorithm, and calibrated with manual scanlines and high-resolution panoramic photographs. Column-forming fractures dominate the fracture system, are either subhorizontal or sub-vertical (i.e., sub-parallel or sub-perpendicular to the brecciated margins) without mean strike orientation, and have an exponential length distribution. Sub-horizontal, clustered platy fractures sub-parallel to the flow direction arrest or deflect column-forming fractures. Areal and volumetric fracture intensity analyses reveal a $\sim 0.5 \%$ connected fracture volume which, although seemingly small, promotes fluid flow due to the planarity and connectivity of the system. Autobreccias are partially connected to column-forming fractures, and may promote lateral flow or form barriers depending on the extent of post-cooling alteration and mineralisation. Discrete fracture network models generated with the measured geometrical parameters are in agreement with the observed highly connected fracture system.

Fractures in the andesite-hosted Rotokawa Geothermal Field are described in cores and BHTV logs. Fractures interpreted on BHTV logs are separated into sets of similar orientation using quantifiable clustering algorithms. Fracture thickness and spacing probability distributions are estimated from maximum likelihood estimations applied to truncated distributions, taking sampling biases into consideration. Spacing of the predominant sub-vertical NE-SW-striking fracture set, and subordinate NW-SE-striking 
fracture set, are best approximated by log-normal distributions and interpreted to be controlled by stratifications within the lava flow sequence. By contrast, spacing of other subordinate fracture sets, either dipping $60^{\circ}$ and striking NE-SW, or steeply dipping and striking N-S, are best approximated by power-law distributions and interpreted to be fault-controlled. Fracture thicknesses in both cores and BHTV logs are approximated by a single power-law distribution, which reflects heterogeneous pathways observed at reservoir scale. Previously reported $\sim 5 \mu \mathrm{m}$-thick fractures studied in thin section do not follow this power-law distribution and have an isotropic orientation, which suggests a change of controls on fracture density and orientation from thermal stresses at thin-section scale, to tectonic and lithological at core and BHTV log scales. However, fractures occupy $\sim 5 \%$ of the rock mass at the three scales of observations, suggesting a self-similar behaviour of fracture volumes in 3-D.

In contrast to the Ruapehu and Rotokawa reservoir studies, scientific drilling in 2014 of the DFDP-2B borehole offered a unique opportunity to investigate the foliation and fractures along a $630 \mathrm{~m}$-long borehole section in metamorphic rocks in the hangingwall of the Alpine Fault. BHTV log interpretation reveals a constant foliation and foliation-parallel fracture orientation $\left(60^{\circ} / 145^{\circ}\right.$; dip magnitude/dip direction) similar to nearby outcrops and parallel to the regional strike of the Alpine Fault. This foliation orientation may reflect the orientation of the Alpine Fault at $\sim 1 \mathrm{~km}$ depth. In addition, sub-vertical fractures striking NW-SE above $\sim 500 \mathrm{~m}$, and sub-horizontal fractures between $\sim 500-820 \mathrm{~m}$ below ground, are interpreted as exhumation-related joints and inherited hydrofractures respectively. Finally, we recognise metre-thick fault zones similar to those identified from BHTV logs and cores in the nearby DFDP-1B borehole. The three fracture set orientations, and observed fault zones, promote high hydraulic connectivity in the Alpine Fault hangingwall, which fosters fluid flow.

This thesis helps quantify the geometrical parameters of fractures and their associated uncertainties in non-sedimentary settings, which are required to constrain numerical models and unravel fluid flow pathways in heterogeneous rocks. We identified lithological, tectonic and thermal controls on fracture geometries, which can constrain conditions and processes by which these fractures formed, and improve the prediction of fracture system architecture away from sparse borehole observations. The results of this thesis are relevant to similar lithological and tectonic settings elsewhere where observations are scarce. This study has also yielded an essential fracture dataset for better understanding of the structural and hydrological conditions at depth near the Alpine Fault prior to a large earthquake. 


\section{Acknowledgments}

This $\mathrm{PhD}$ has been a long journey, with a lot of people to thank. First are my three supervisors: Dave, we have had a long productive and enthusiastic time together, which I know will continue through space and time. Andy was always ready to listen to me, and his long experience in cracks strengthened all interpretations. John continuously brought me new perspectives, not the least by bringing me into the DFDP team, and hammering that my fonts were too small. I plead guilty of any harassment complaint you would lodge, and I am amazed by how much time you have given me. I still have a lot to learn from you, and will do my best to keep the links solid - though less intensively, be reassured! Comments from the three examiners (Nicholas Davatzes, Julie Rowland and Martha Savage), also improved this thesis.

GNS Science has a lot to do with this project: bringing me to New Zealand to work with the "top DOGS" (Go team!), giving me opportunities, helping me to navigate the system, and generally exposing me to data and research which makes $3 / 4$ of this PhD. More pragmatically, I am grateful to GNS for awarding me the Sarah Beanland Memorial Scholarship, which supported me financially and logistically beyond the scholarship, with enough freedom to make this project my own. The TLS study would simply not have been possible without Pilar Villamor. I enjoyed the sciency (or not) discussions with the DOGs, BITs and Supermodelers, and I'm looking forward coming back fully into the team(s). The WING group already empowered me for the great battle of gender equity in an industry I care for - more to come.

Thanks also to the Department of Conservation, Ngati Rangi and Ngati Hikairo iwi, and Ruapehu Ski Lifts for access to the outcrop. I enjoyed my encounters with Ngati Rangi - I will keep learning about the values of your people. Thanks are extended to Golder Associates for availability of the Fracman software. I am grateful to Mercury New Zealand and the Rotokawa Joint Venture who provided the geothermal data, and discussions on the reservoir behaviour.

VUW hosted me, cared for me (thanks to all the staff!), taught me valuable skills to be a better researcher (Lizzie Towl in particular), funded trips to conferences and exposed me to a variety of topics: Geophysics and Geosciences, but also Antarctic research, climate change or architecture. I learned a lot about wiggles with the geophysics group. Thanks to Euan Smith and our neighbour Richard Arnold for making stats accessible.

Joining the DFDP science team has been fabulous, whatever I said about the joys 
of being stuck at Whataroa being devoured by sandflies. That was my second scientific drilling, and I can't wait for the next one. Mai-Linh: you are simply the most enthusiastic and efficient person I've ever met, and a role model. The Wellcad parties were intense but productive and fun, and a few days work with you equals weeks on my own. Bernard, with his jokes of questionable taste, pickyness and scepticism, improved my work and writing significantly, and it was also great to share tricks with another BHTV geek. Others from the team (Rupert, Ray, Tim, Doug, Tamara, Jack, Lucie, Jamie and others) made the drilling enjoyable and a big learning experience.

Amongst other collaborators, I will name: Garth Archibald (GNS), David GarciaSelles at University of Barcelona, Paul Siratovich, Marlène Villeneuve and Jonathan Davidson at University of Canterbury, Chris Conway, Tim Little, Jack Williams, the Gocad group, BRGM, Geosciences Rennes (finally made it into my home town university!); I'm looking forward more collaborations and trips.

On the lighter side, but also critical, are my officemates: the first ones, Calum, Emily and Jesse, abandoned me recently but also proved it's all possible; and the more recent ones, Kostas and Dominic, provided singing and 'mericanness. With the rest of the students, they filled this time with laughter, support and beverages, and it was nice to have another "arrogant" French around (Laura-May) to get excited about real bread. Thanks Jesse and Dom for counting cracks with me. The VUWTC is the best bunch of crazies I know of. There, I found people to indulge in sillyness, tramping which pushed my limits, and good spirits. Kerry (I don't thank you for mispronouncing my name), Maaarc and Meeeredith give the best hugs. Âchim told me when I needed it that it was worth the efforts to use MLE; he also supported me through this endeavour, and questioned my ideas about fitness levels. Tacos has been my breath of air every Tuesday. Apparently I'm going to be quite involved in this club from now on, and I know it's going to be fun (all types included). Kyle taught me how to kiwi, and proved it's worth following dreams. In a strange way, thanks to -Max- and Alex who instilled the PhD idea. Thanks Philipp for the TLS suggestion on a trip up Ruapehu. Smiiix to Léa, Ludi and Yohan - we have very different lives, but we still connect. Special thanks to the Geool crew (Johan, Anne-so, Elo, Theo and co); we shall catch-up somewhere some time, and it will be fun. Wellington is a cool and motivating place to live in, which also kept me vaguely fit with this damn hill twice a day.

Thanks to my family; they kept me grounded on what the other $99 \%$ of people care about.

Finally, thanks Kieran. You make me happy everyday. That's a rare gift. Thanks also for organising my holidays, distracting me, feeding me, pushing my limits, believing in me, hiding chocolate, proof-reading, cooking the best tramping food, putting up with me (especially these past few months); I plan to redeem myself a bit, though not as far as enjoying cricket! 


\section{Contents}

Abstract

Acknowledgments

List of Figures $\quad$ xi

$\begin{array}{ll}\text { List of Tables } & \text { xv }\end{array}$

1 Introduction 1

1.1 Motivations . . . . . . . . . . . . . . . . . . . . . . . . 1

1.2 Aims of this Study . . . . . . . . . . . . . . . . . . 3

1.3 Fracture Systems: Processes, Description by Scaling Laws, and Modelling 4

1.3.1 Controls on Fracture System Generation and Permeability . . . 4

1.3.2 Statistical Description of Fracture Sets: Tools to Describe Fracture Geometries and Effects on Permeability . . . . . . . . . . 6

1.3.3 Fracture Models . . . . . . . . . . . . . . . . . . . . . . . . . 9 9

1.4 Geological Settings of the Three Case Studies . . . . . . . . . . . . . . 10

1.4 .1 New Zealand Plate Boundary . . . . . . . . . . . . . . . . . . 11

1.4 .2 Ruapehu Volcano . . . . . . . . . . . . . . . . . . . . . . . . . . 11

1.4 .3 Rotokawa Geothermal Field . . . . . . . . . . . . . . . . . . . . 11

1.4.4 Central Alpine Fault, Deep Fault Drilling Project (DFDP) . . . 13

1.5 Data . . . . . . . . . . . . . . . . . . . . . . 14

$1.5 .1 \quad$ Outcrop Datasets $\ldots \ldots \ldots$. . . . . . . . . . . . . . . 14

1.5 .2 Borehole Datasets . . . . . . . . . . . . . . . . . . . . 16

1.6 Thesis Content . . . . . . . . . . . . . . . . . . . . . . . . . . 17

2 Quantitative Geometric Description of Fracture Systems in an Andesite Lava Flow Using Terrestrial Laser Scanner Data 21

2.1 Introduction . . . . . . . . . . . . . . . . . . . . . . . 22

2.2 Geological Setting . . . . . . . . . . . . . . . . . . . . . 24

$2.2 .1 \quad$ Ruapehu volcano, Central North Island of New Zealand . . . . . 24

$2.2 .2 \quad$ Description of the Happy Valley Outcrop . . . . . . . . . . . . . 24

2.3 TLS Processing, Fracture Processing and Fracture Analysis . . . . . . . 26

$2.3 .1 \quad$ Data Acquisition . . . . . . . . . . . . . . . . . 26 
$2.3 .2 \quad$ Fracture delineation From the TLS Point Cloud . . . . . . . . . 28

2.3 .3 Sampling Bias . . . . . . . . . . . . . . . . . . . . . . . . . . . . 28

2.3 .4 Fracture Analysis Method . . . . . . . . . . . . . . . . . 30

2.4 Results: Fracture Geometries in the Happy Valley Outcrop . . . . . . . 33

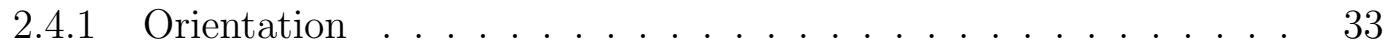

2.4 .2 Connectivity (Scanline) and Length (TLS and Scanline) . . . . 35

2.4.3 Variability of Fracture Area Between Breccia and Blocky Facies 38

$2.4 .4 \quad$ Linear Fracture Intensity: $\mathrm{P}_{10} \ldots \ldots \ldots$

2.4.5 Areal $\left(\mathrm{P}_{21}\right)$ and Volumetric $\left(\mathrm{P}_{32}\right)$ Intensity, and Fracture Porosity $\left(\mathrm{P}_{33}\right) \ldots \ldots \ldots \ldots \ldots \ldots$

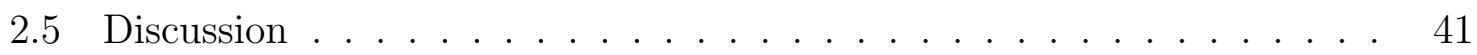

2.5.1 Applicability of TLS for Fracture Characterisation in Volcanic Rocks ....................... . . 41

2.5 .2 Column-forming Fracture Orientation and Size . . . . . . . . . . 42

2.5 .3 Column-forming Fracture Intensity . . . . . . . . . . . . . . . 43

2.5.4 Controls on the Geometry of Column-forming Fractures. . . . . 44

2.5 .5 Upscaling Fracture Geometries to Volcano scale . . . . . . . . . 45

2.5.6 Potential Effects of the Fracture System on Reservoir Permeability 46

2.5.7 Fracture System Modelling for Happy Valley-type Lava Flows . 47

2.6 Conclusion . . . . . . . . . . . . . . . . . . . . . . . . . . . . . . . . . 50

\begin{tabular}{ll}
\hline Appendices & 52
\end{tabular}

2. A $\quad$ TLS Processing Workflow . . . . . . . . . . . . . . . . . . . . . 52

2.B Fracture Radius Parameters . . . . . . . . . . . . . . . . . . . 54

3 Statistical Methods of Fracture Characterisation Based on Acoustic Borehole Televiewer Log Analysis 55

3.1 Abstract . . . . . . . . . . . . . . . . . . . . . 55

3.2 Introduction . . . . . . . . . . . . . . . . . . . . . . . 55

3.3 Data . . . . . . . . . . . . . . . . . . . . . 57

3.4 Fracture Set Orientation Clustering . . . . . . . . . . . . . . . . . 58

$3.4 .1 \quad$ Cluster Analysis Methods . . . . . . . . . . . . . . . . . . 58

$3.4 .2 \quad$ Fracture Set Delineation Example . . . . . . . . . . . . . . . . 61

3.5 Probability Distribution Analysis of Fracture Attributes . . . . . . . . . 63

3.5.1 Sampling Biases . . . . . . . . . . . . . . . . . . . 63

3.5.2 Effects of BHTV Log Quality . . . . . . . . . . . . . . . 65

3.5.3 Maximum Likelihood Estimation Using Truncated Distributions 65

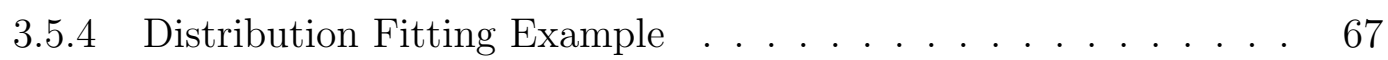

3.5.5 How Many Fractures Are Enough? . . . . . . . . . . . . . . . . 69

3.6 Discussion . . . . . . . . . . . . . . . . . . . . . . . . 71 
3.6.1 Sampling Effects Considerations for the Statistical Analysis of Fractures Interpreted in BHTV Logs . . . . . . . . . . . 71

3.6 .2 Uncertainties, and Multiple "Best" Solutions . . . . . . . . . . . 72

3.6 .3 Multivariate Analysis . . . . . . . . . . . . . . . . . . 73

3.7 Conclusion . . . . . . . . . . . . . . . . . . . . . . . . . 74

\begin{tabular}{ll}
\hline Appendices & 75
\end{tabular}

3.A Evaluation of the Number of Orientation Clusters for Each Borehole . . $\quad 75$

3.B Probability Distribution Formulas . . . . . . . . . . . . . . . . . 76

4 Tectonic, Lithologic and Thermal Controls on Fracture System Geometries in an Andesitic High-Temperature Geothermal Field $\quad 77$

4.1 Abstract . . . . . . . . . . . . . . . . . . . . . . . 77

4.2 Introduction . . . . . . . . . . . . . . . . . . . . . . . . 78

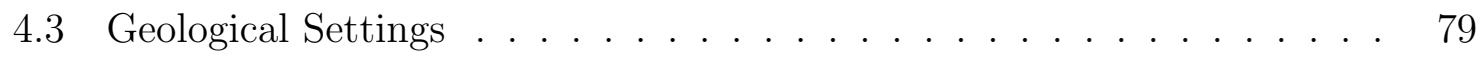

4.4 Data $\ldots \ldots \ldots \ldots \ldots$. . . . . . . . . . . . . . . . . . . . . . . 81

4.4 .1 Cores . . . . . . . . . . . . . . . . . . . . . . . . . . . . . . 81

$4.4 .2 \quad$ BHTV Logs . . . . . . . . . . . . . . . . . . . . 82

4.5 Method . . . . . . . . . . . . . . . . . . . . . . 85

$4.5 .1 \quad$ Fracture Processing . . . . . . . . . . . . . . . . . 85

4.5 .2 Distribution Fitting . . . . . . . . . . . . . . . . . . . . . 85

4.5 .3 Density Calculations in Cores and BHTV Logs. . . . . . . . . . 86

4.6 Results . . . . . . . . . . . . . . . . . . . . . . . . . . . . . . 87

4.6 .1 Orientation . . . . . . . . . . . . . . . . . . . . 87

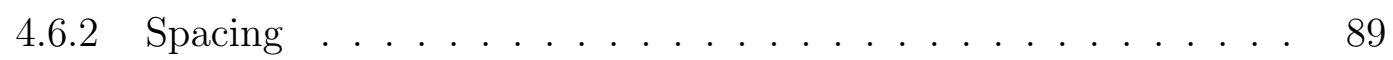

4.6 .3 Thickness . . . . . . . . . . . . . . . . . . . . . . . . 91

4.6 .4 Density . . . . . . . . . . . . . . . . . . . . . 92

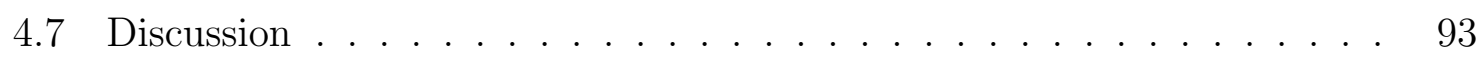

4.7 .1 Spacing and Orientation . . . . . . . . . . . . . 93

4.7 .2 Thickness . . . . . . . . . . . . . . . . . . . . . . . . . . . . . 95

4.7 .3 1-D and 3-D Fracture Abundances . . . . . . . . . . . . . . . 96

$4.7 .4 \quad$ Effect of Lithology and Alteration on Fluid Pathways . . . . . . 98

4.7 .5 Potential Controls on Structural Permeability . . . . . . . . . . 98

4.7 .6 Modelling Rotokawa Fracture Systems at Reservoir Scale . . . . 99

4.8 Conclusion $\ldots \ldots \ldots \ldots$. . . . . . . . . . . . . . . . . . . . . . . . . . . . . . . . . 100

\begin{tabular}{ll}
\hline Appendices & 102
\end{tabular}

4. A Results of Probability Distribution Analysis . . . . . . . . . . . . . . . 102 
5 The Alpine Fault Hangingwall Viewed From Within: Structural Analysis of Acoustic Image Logs in the DFDP-2B Borehole, New Zealand 105 5.1 Abstract . . . . . . . . . . . . . . . . . . . . . . . . . . . . . 105

5.2 Introduction . . . . . . . . . . . . . . . . . . 106

5.3 Geological settings $\ldots \ldots \ldots$. . . . . . . . . . . . . . . . . . . 108

5.4 Borehole Televiewer (BHTV) Log Processing and Analysis Methods . . 109

$5.4 .1 \quad$ BHTV Logging and Processing . . . . . . . . . . . . . . . . 109

$5.4 .2 \quad$ BHTV Log Quality . . . . . . . . . . . . . . . . . . . . . 110

5.4 .3 Characterisation of Planar Features . . . . . . . . . . . . . . . . 112

$5.4 .4 \quad$ Acoustic Image Facies Delineation . . . . . . . . . . . . . . . . 113

5.5 Results . . . . . . . . . . . . . . . . . . . . . . . 114

5.5 .1 Planar Feature Orientation . . . . . . . . . . . . . . . . . . . 114

$5.5 .2 \quad$ Planar Feature Density . . . . . . . . . . . . . . . . . . . . . 114

5.5.3 Relationships Between Planar Feature Appearance, Morphology and Orientation . . . . . . . . . . . . . 116

5.5 .4 Planar Feature Thickness . . . . . . . . . . . . . . . . . 116

5.5.5 Low Amplitude Zones and Acoustic Image Facies . . . . . . . . 117

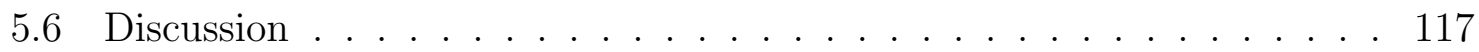

5.6.1 Feature Set 1 (FS1): Foliation Planes and Foliation-parallel Fractures . . . . . . . . . . . . . . . . . 117

5.6.2 Foliation Orientation: Implications for the Alpine Fault Geometry 119

5.6.3 FS2 and FS3: Joints Formed During Exhumation, and Inherited Hydrofractures . . . . . . . . . . . . . . . . . . . 121

5.6.4 Possible Subsidiary Fault Zones in the Alpine Fault Hangingwall 123

5.6 .5 Interpretation of the Acoustic Image Facies. . . . . . . . . . . . 124

5.6 .6 Perspectives: Implications for Permeability . . . . . . . . . . . . 125

5.7 Conclusion $\ldots \ldots \ldots \ldots \ldots$. . . . . . . . . . . . . . . . . . . . . 127

\begin{tabular}{lr}
\hline Appendices & 128
\end{tabular}

5.A BHTV Log Acquisition and Processing . . . . . . . . . . . . . . . . . . 128

5.B Calculations of Borehole Diameter (Caliper) . . . . . . . . . . . . . . . 128

5.C Impact of Drilling and Logging on BHTV Log Quality . . . . . . . . . 131

$\begin{array}{lll}6 & \text { Synthesis } & 135\end{array}$

6.1 Main contributions . . . . . . . . . . . . . . . . . . . . . . . . 135

$6.1 .1 \quad$ Key Results of Each Chapter . . . . . . . . . . . . . . . . . 135

6.1 .2 Key Contributions to the Research Questions . . . . . . . . . . 137

6.2 Implications for Future Research . . . . . . . . . . . . . . . . . . . 143

6.2.1 Role of Past Geological History on Current Fracture System Geometry . . . . . . . . . . . . . . . . . . . . . . 143 
6.2.2 From Observed Geometry to Connectivity, Stress and Fluid Flow 145 $6.2 .3 \quad$ Finding Constraints on Permeability . . . . . . . . . . . . . . . 148

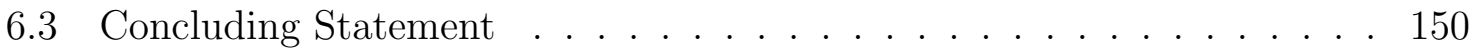

\begin{tabular}{ll}
\hline Appendices & 151
\end{tabular}

\begin{tabular}{|l|l|}
\hline A Physical Properties of Ruapehu Volcano Samples & 152
\end{tabular}

A.1 Methods . . . . . . . . . . . . . . . . . . . . . . 152

A.1.1 Density and Connected Porosity Measurements . . . . . . . . . 152

A.1.2 Characterisation of Elastic Wave Velocities and Dynamic Elastic Moduli . . . . . . . . . . . . . . . . . 153

A.1.3 Uniaxial Compressive Strength Testing and Static Elastic Moduli Calculations .................... 153

A.2 Results . . . . . . . . . . . . . . . . . . . . . . . . . . . . . . . 154 


\section{List of Figures}

$1.1 \quad$ Fracture and fault structure in layered rocks $\ldots \ldots \ldots \ldots$

$1.2 \quad$ Schematic fracture and fluid flow models in layered jointed rocks and fault damage zones $\ldots \ldots \ldots \ldots$. . . . . . . . . . . . . . . . 7

$1.3 \quad$ Effect of variable fracture spacing and aperture on heat extraction . . . 8

1.4 New Zealand's main tectonic features and TVZ's main geothermal systems 12

1.5 Scale and dimensionality of sampling methods used in this thesis. . . . 14

1.6 Data used in this thesis . . . . . . . . . . . . . . . . . 15

2.1 Location of the "Happy Valley" lava flow outcrop. . . . . . . . . . . 25

2.2 Detection limits of the TLS and factors influencing the column-forming fracture propagation $\ldots \ldots \ldots \ldots \ldots \ldots \ldots \ldots \ldots$

$2.3 \quad$ Flowchart of the TLS data processing and fracture analysis . . . . . . 29

2.4 Schematics of fracture measurements on the manual scanline, TLSprocessed fractures, and trace maps . . . . . . . . . . . . . 30

2.5 Fracture delineation of the TLS dataset: high-resolution panoramic photograph with manual scanline, TLS point cloud, and delineated fracture orientation and area . . . . . . . . . . . . . . . . . . . 31

2.6 Empirical probability density function of dip magnitude and length for the scanline and TLS-processed datasets $\ldots \ldots \ldots \ldots . \ldots 35$

2.7 Orientation of column-forming fractures in relation to outcrop orientation 36

2.8 CCDF of fracture length for fractures measured on the scanline and TLS-processed dataset $\ldots \ldots \ldots \ldots$. . . . . . . . . . . . 37

2.9 Variation of fracture area between the blocky and upper brecciated zones 39

2.10 Empirical PDF of linear fracture density $\left(\mathrm{P}_{10}\right)$ along a series of virtual scanlines . . . . . . . . . . . . . . . . . . . . . . . . . . 39

2.11 Measured $\mathrm{P}_{21}$ (areal intensity) and estimated $\mathrm{P}_{32}$ (volumetric intensity) and $\mathrm{P}_{33}$ (fracture porosity) parameters $\ldots \ldots \ldots \ldots \ldots$

2.12 Interpretive diagram of the Happy Valley lava flow outcrop . . . . . . . 44

2.13 Examples of DFN models $\ldots \ldots \ldots \ldots \ldots$

2.A.1Fracture processing of the TLS point cloud . . . . . . . . 53

$3.1 \quad$ Example of BHTV $\log \ldots \ldots \ldots \ldots \ldots \ldots$

3.2 Evaluation of the optimal number of cluster. . . . . . . . . 60

3.3 Example of Fisher, K-means, fuzzy K-means and agglomerative clustering 62 
3.4 Sampling bias limits for fracture thickness and spacing . . . . . . . . . 64

3.5 Impact of BHTV $\log$ quality on the data to fit . . . . . . . . . . . . . 66

3.6 Spacing and thickness distribution fitting . . . . . . . . . . . . . . 68

3.7 Number of fitted distributions satisfying the AIC and SBC conditions as a function of the input number of data points and scale range . . . . 69

3.8 Fitting of a spacing dataset within a series of truncated limit intervals . $\quad 70$

3.9 Q-Q plots of fitted distributions . . . . . . . . . . . . . . . . . 73

3.A.1Evaluation of the optimal number of cluster for all boreholes . . . . . . 75

$4.1 \quad$ Schematic fracture and fluid flow models in layered jointed rocks and fault damage zones . . . . . . . . . . . . . . . . . . . . . . . . . . 79

4.2 Location map of the Rotokawa Geothermal Field . . . . . . . . . . . . 80

4.3 Lithologies, alteration and fracturation observed in cores from the Rotokawa Geothermal Field . . . . . . . . . . . . . . . . . . . . . . . . . . 82

4.4 Example of BHTV $\log$ and fracture measurements . . . . . . . . . . . . 84

4.5 Fracture set orientations in the Rotokawa andesites . . . . . . . . . . 88

4.6 Selected examples of spacing distribution fits . . . . . . . . . . . . . . . 90

$4.7 \quad$ Fracture thickness distribution in BHTV logs and cores . . . . . . . . . 92

4.8 Fracture density in cores . . . . . . . . . . . . . . . . . . . . . . . . . . 93

$4.9 \quad$ Multiscale $\mathrm{P}_{10}$ and $\mathrm{P}_{33} \ldots \ldots \ldots \ldots \ldots$

$5.1 \quad$ Map with DFDP-2 and DFDP-1 boreholes, Alpine Fault and nearby townships locations . . . . . . . . . . . . . . . . . 107

5.2 Schematic cross-section along the Whataroa Valley . . . . . . . . . . . 109

5.3 Composite of BHTV log interpretation and other wireline logs used in this study . . . . . . . . . . . . . . . . . . . . . . 111

5.4 Feature morphology and descriptors . . . . . . . . . . . . . . . . 112

5.5 Orientation of features identified in the BHTV logs in DFDP-1B and DFDP-2B boreholes . . . . . . . . . . . . . . . . . . . . . . 115

5.6 Appearance of acoustic image facies and low amplitude zones . . . . . . 118

$5.7 \quad$ Photographs of foliated Alpine Schists, with fractures and veins interpreted to be similar to those in the BHTV log . . . . . . . . . . . . 120

5.8 BHTV log of the 710-750 m interval surrounding a major static temperature gradient change . . . . . . . . . . . . . . . . . . . . . 124

5.9 Examples of permeable fractures identified by temperature changes . . 126

5.B.1Example of caliper calculation from BHTV log . . . . . . . . . . . . . 130

5.C.1Impact of drill bit type on BHTV $\log$ appearance . . . . . . . . . . . . 132

6.1 Possible evolution of volcanic rocks through depth and time . . . . . . 140

6.2 Effect of fractures on flow . . . . . . . . . . . . . . . . . 146

6.3 Effects of fracture orientation on stress and fluid flow under extension . 148 
A.1 Crossplots of physical properties of Ruapehu samples . . . . . . . . . . 155

A.2 $\quad$ Stress-strain behaviour and UCS of Ruapehu samples . . . . . . . . . . 156 


\section{List of Tables}

2.1 Geometrical parameters of column-forming fractures measured from the manual scanline and TLS-processed datasets, and parameters used to model DFN models . . . . . . . . . . . . . . . . . . . . . . . . . 34

2.B.1Parameters of the probability distribution fitted to the fracture radius . 54

3.B.1Probability density function formulas of distributions used in this thesis 76

$4.1 \quad$ Fracture density in core, BHTV logs and thin-section . . . . . . . . . . 83

4.A.1Results of probability distribution analysis of fracture thickness . . . . 103

4.A.2Results of probability distribution analysis of fracture spacing . . . . . 104

5.1 Appearance of the planar features . . . . . . . . . . . . . . . . 113

5.2 Morphology of the planar features . . . . . . . . . . . . . . . . . . . . . 113

5.3 Number and orientation of planar features for each fracture set, characterised by their appearance, morphology and planarity . . . . . . . . . 114

5.A.1List of BHTV logs used in this study . . . . . . . . . . . . . . . . . 129

A.1 Physical property measurements of samples from Happy Valley lava flow and Meads Wall dyke . . . . . . . . . . . . . . . . . . . 155 


\section{Introduction}

\subsection{Motivations}

This thesis is motivated by two key aspects: 1) There is sufficient geothermal energy beneath $\sim 2.3 \%$ of New Zealand's land area to provide electricity for the entire country, with low greenhouse gas emissions and impacts on the landscape (Bignall, 2010; Ministry of Business Innovation and Employment, 2016); 2) The Alpine Fault, a plate bounding structure of New Zealand, is late its interseismic cycle and forecast to rupture in a large earthquake in the near future, which may cause significant damage Berryman et al., 2012, Nicol et al., 2016). Although these two aspects may seem unrelated, they are both linked to fluid flow in fractured rock masses. Fractures ${ }^{1}$ strongly influence the flow of fluids in the crust, modifying the hydrological and stress conditions on active faults, which can beneficially increase permeability Barton et al., 1995; Townend and Zoback, 2000; Barton et al., 2013) and facilitate the economic development of geothermal resources (Grant, 2016), but can also promote earthquake generation (Sibson (1992); Hickman et al. (1995) and references therein; Zoback et al. (2007); Sutherland et al. (2007)).

Advances in unravelling the controls that fractures exert on reservoir permeability have risen in recent years due to the increased development of high-temperature fractured geothermal reservoirs $($ Bertani, 2016) and enhanced geothermal systems (Breede et al., 2013); hydrocarbon extraction from fractured carbonates, low-porosity sedimentary and volcanic rocks (Feng, 2008; U.S. Energy Information Administration, 2014); storage of unwanted $\mathrm{CO}_{2}$ (Pollyea and Fairley, 2012) and radioactive waste (Chen et al. 2001); and effects of fluids on both natural and man-made seismicity (Ellsworth, 2013). However, correlations between fracture system geometrical attributes (such as orientation, aperture and length) and permeability in rock masses are not always clear (Nemčok et al. 2004, McNamara et al., 2015). While fracture systems have been extensively studied in sedimentary and plutonic rocks (e.g., Barton et al., 1995), few studies have been devoted to volcanic and metamorphic rock masses.

In this thesis, we aim to quantify the geometry of fracture systems and its potential impact on permeability in three settings, which each have wider applications: an andesitic lava flow on an active volcano, an andesite-hosted geothermal reservoir, and the

\footnotetext{
${ }^{1}$ In this thesis, fractures include joints with dilation only, faults with shear displacement, and veins which have mineral infill and accommodate shear and/or dilation.
} 
metamorphic hangingwall of the Alpine Fault. In volcanic rocks, these applications include assessment and mitigation of volcanic hazards (Voight, 2000), engineering (Koca et al., 2011) and quarrying of volcanoes; geothermal resource utilisation (Bertani, 2016; Lund and Boyd, 2016) and $\mathrm{CO}_{2}$ sequestration (Pollyea et al., 2014) for sub-surface volcanic rocks; and mining of epithermal deposits after exhumation (Christie et al., 2007). Andesite-hosted geothermal reservoirs are common worldwide, and are currently in operation or being explored in several countries including Indonesia, Nicaragua and Chile (Lahsen et al., 2015, and references therein). Similarly, characterising fracture systems in metamorphic rocks near the Alpine Fault has practical applications in earthquake hazard evaluation (Townend et al., 2009; Berryman et al., 2012) and possible geothermal use due to the high thermal gradient (Sutherland et al., 2015, submitted).

Fracture systems in the vicinity of large faults such as the Alpine Fault in the South Island of New Zealand have been extensively studied (e.g., Sibson, 1992, Caine et al., 1996; Williams et al., 2016) but local, site-specific studies are still necessary to delineate fluid flow pathways. Similarly, permeability in fracture-controlled geothermal reservoirs is strongly affected by large faults and their damage zones (Arnórsson, 1995; Nemčok et al., 2007; Benato et al., 2016; McNamara et al., 2016a). Faults can act as conduits or barriers (Caine et al., 1996; Rowland and Sibson, 2004), and can also have different permeability depending on the tectonic regimes they were formed under, as shown in the Rhine Graben (France; Dezayes et al., 2015). Drilling of the DFDP-2B borehole in 2014 offers the first opportunity to study the fracture system in the hangingwall of the Alpine Fault at depth, and thus to complement detailed fracture studies conducted on outcrops (e.g., Gillam et al., 2013), and indirect seismological observations (e.g., Boese et al. 2012). DFDP-2B drilling also provided data on rarely studied fracture systems in foliated, metamorphic rocks. Because of the superposition of fault-related deformation onto the schist lithology, borehole data provides a "snapshot" of the fracture system at an intermediate stage of exhumation, and may reveal a combination of features specific to the original schist lithology as well as fault-related and exhumation-related structures.

Geothermal reservoirs hosted in volcanic formations have a complex fracture history, starting with fractures and breccias formed during lava emplacement (e.g., DeGraff and Aydin, 1987; Spörli and Rowland, 2006), with a spatial variability at the scale of the entire volcano due to the variety of volcanic products. After burial, fractures are associated with the development of faults and their damage zones (e.g., Johri et al., 2014), due to contemporary tectonics, or resulting from reactivation of inherited basement faults Seebeck et al. 2014a; McNamara et al., 2015). Fluid circulations during the active life of a geothermal system may deposit ore minerals Krupp and Seward, 1987; Cox, 2016, Simmons et al. 2016) and develop into epithermal deposits after exhumation, such as those found in Coromandel, New Zealand Brathwaite et al., 2001. Christie et al., 2007). Geothermal systems are themselves transient features which can 
result from successive magmatic pulses (Chambefort et al., 2014; Milicich et al., 2016), or become extinct (Kissling et al., 2015a). Above-ground exposures, either of volcanoes or epithermal deposits, thus represent two possible end-members in the history of volcanic-hosted geothermal systems. While remnants of joints, flow-banding and autobreccias formed during the emplacement of volcanic rocks are observed in places in epithermal deposits (Brathwaite et al., 2001; Zuquim and Rowland, 2013), it is not clear why this inheritance is not always preserved, nor how processes occurring during the geothermal stage conserve or obliterate the original volcanic features. Refining the links between fractures in volcanic formations, volcanic-hosted geothermal systems and volcanic-hosted epithermal systems is thus important for understanding how geothermal systems evolve temporally. This also has practical consequences for managing geothermal resources hosted in volcanic rocks.

\subsection{Aims of this Study}

This thesis focuses on the evaluation of geometries of fracture systems, the processes which control these geometries, and the potential effects of fracture geometry on permeability. This thesis also discusses avenues for modelling such fracture systems. The main research question addressed in this thesis is:

What is the architecture of the fracture system in non-sedimentary layered fractured rock masses conducting hydrothermal fluids? To date, the majority of fracture system geometrical analysis has been dedicated to sedimentary settings, rather than volcanic and metamorphic settings. This question will be addressed through three case studies: (1) an outcropping andesite lava flow, (2) an andesitehosted high-temperature geothermal field, and (3) the metamorphic hangingwall of the Alpine Fault. We examine fracture geometries in outcropping andesite lava flows on the flanks of the Ruapehu volcano (Chapter 2). These geometries are used as a reference for the fracture system of the andesite-hosted Rotokawa geothermal reservoir prior to burial, hydrothermal alteration, and tectonic activity. Fracture geometries in the present-day Rotokawa geothermal reservoir are directly studied from borehole data (Chapter 4). Finally, we study fracture geometries from BHTV log observations in the metamorphic hangingwall of the Alpine Fault in the context of larger scale outcrop observations (Chapter 5), which provides additional information on fracture systems in foliated rocks near a large active fault.

In addition, three key underlying research questions guide the analysis throughout the thesis:

1. How can we improve the analyses of fracture geometries? This work utilises a number of datasets, including terrestrial laser scanner (TLS) point clouds, 
outcrop scanlines, borehole acoustic televiewer (BHTV) logs, and cores. We assess and address sampling biases specific to each dataset. To complement the traditional and largely subjective fracture orientation analysis from stereonets, we use clustering algorithms to provide objective classifications of fracture sets (Chapter 3). We use the maximum likelihood estimation method applied to truncated distributions to estimate the forms and parameters of probability distributions of fracture attributes, which can then be used as inputs into fracture models. In addition, we advance the TLS fracture analysis workflows to automatically extract and analyse fracture geometries from an entire outcrop (Chapter 2). Finally, we combine different datasets at various scales to strengthen our interpretation in hydraulic and geomechanical terms.

2. What controls the fracture network organisation in fractured rock masses? An underlying theme of this thesis is the evaluation of possible lithological (such as rock type, layering and alteration), tectonic stress, and hydrothermal (involving thermal stresses and precipitation/dissolution of minerals) controls on the geometry of the fracture system and associated permeability.

3. How can we model fracture systems in these rock masses? A practical aspect of this thesis involves providing geologically-informed probability distributions of fracture geometrical parameters that can constrain kilometre-scale fracture and fluid flow models. Given the analysed fracture geometries, we suggest relevant modelling techniques and propose methods to mitigate uncertainties.

\subsection{Fracture Systems: Processes, Description by Scaling Laws, and Modelling}

A fracture system results from the interaction of complex processes affecting discontinuities. In this section, we describe the dominant processes affecting the geometry of fracture systems and the scaling laws which result from these processes and can summarise complex observations. We also present some techniques that have been used to model fracture networks.

\subsubsection{Controls on Fracture System Generation and Perme- ability}

The geometry of fracture systems is controlled by several types of processes:

1. The development of fractures is affected by the rheology and heterogeneity of the host rock type (e.g., limestone or andesite lava; Ladeira and Price, 1981; Aydin and Degraff, 1988; Mc Caffrey et al., 2003), including possible layering (e.g., 
bedding or foliation; Schöpfer et al., 2011; Misra et al., 2015) and petrophysical properties such as tensile strength, porosity and tortuosity Zoback, 2007; Heap and Kennedy, 2016).

2. Tectonic effects, either current or past, cause faulting and preferential opening of fractures (Barton et al. 1995, Rowland and Sibson, 2004; Dezayes et al., 2015).

3. Weathering, diagenesis and hydrothermal alteration can result in compaction, pervasive alteration, and a decrease or increase in porosity (matrix and fracture) depending on mineral precipitation or dissolution (Custodio, 2007), which is intense in hydrothermal systems Sonney and Mountain, 2013, McNamara et al., 2016b).

4. Fluid pressure increases bring fractures closer to failure (Hickman et al. (1995) and references therein; Barton et al. (1995); Cox (1995); Sibson (1996)).

5. Thermal stress effects, which can be negligible in many systems, can be significant in geothermal reservoirs, and active fault zones with high rates of heat and fluid advection such as the Alpine Fault (Siratovich et al., 2014, 2015; Sutherland et al. 2015).

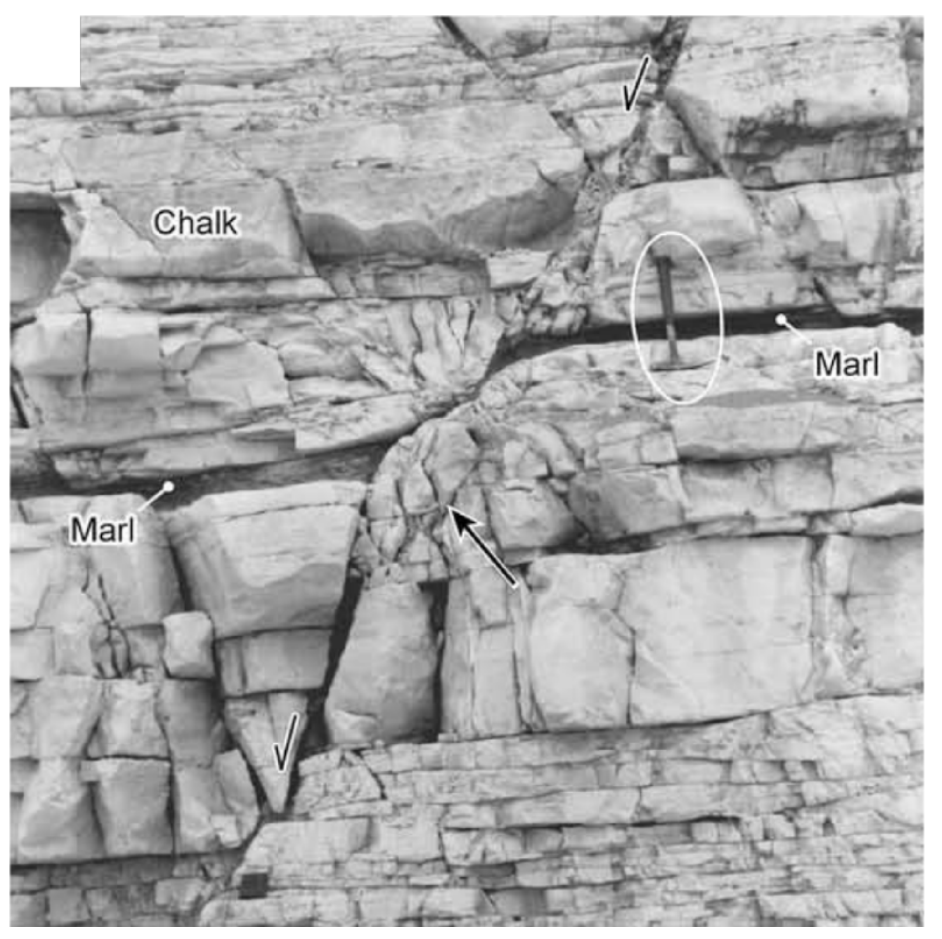

Figure 1.1: Outcrop photograph of a segmented fault step across a thin marl unit, with intense fracturing adjacent to a fault bend (arrow). The chalk layers, which are more competent than the marl layer, are transected by bed-normal, stratabound fractures (from Childs et al., 2009). 
The effects of layering on fracture systems (process 1) are well documented in sedimentary rocks (e.g., Childs et al., 2009) and influence flow pathways. For example, fault segmentation can occur at the interface between layers of different competency (Figure 1.1), with increased permeability potential in the intensely fractured fault bends. In addition, pervasive fracture networks, including stratabound bed-normal fractures in layered sedimentary rocks (Figure 1.1), can produce significant fluid storage volumes, or flow when connectivity is sufficient (Bour and Davy, 1998; Agosta et al., 2010). In the hangingwall of the Alpine Fault, the penetrative foliation and fracture systems exert a significant control on fluid flow (Upton and Craw, 2014); however, observations are nearly exclusively made at the ground surface (e.g., Gillam et al., 2013), and conditions at depth remain unexplored. Volcanic rocks are also layered at different scales, resulting from flow-banding within lava flows, autobreccias intercalated between individual lava flows, and heterogeneous deposits from pyroclastic eruptions Aydin and Degraff, 1988; Conway et al., 2016). The effects of these heterogeneities on the geometry of fracture systems and resulting permeability are not well understood in volcanic-hosted reservoirs and metamorphic rocks.

Fluids are intimately linked to processes $2-5$, in particular in relation to faulting (see review by Hickman et al., 1995; Townend and Zoback, 2000, Zoback et al., 2007), as demonstrated by earthquakes caused by fluid injection ("fracking"; Evans, 2005, Ellsworth, 2013) or post-earthquake groundwater level changes (Cox et al., 2012). In these cases, increases in fluid pressure within a given regional tectonic stress field decrease fault stability, by decreasing the effective normal stress on the fault plane (e.g., Zoback, 2007). On the other hand, structural and mineralogical textures indicate that episodic fracturing and brecciation are followed by cementation and crack healing (Williams et al., 2016), leading to cycles of permeability enhancement and reduction accompanied by episodic fluid flow along faults (e.g., Cox, 1999). The cementation of fractures can generate impermeable barriers and compartmentalise reservoirs Rowland and Sibson, 2004) as observed in the Rotokawa geothermal reservoir Hernandez et al., 2015) which can episodically be ruptured ("fault-valve system"; Sibson, 1992). A careful description of the geometry of the fracture and vein network is thus a precursor to the characterisation of fluid flow in fracture rock masses in a given stress field, and with time-dependent pore pressure and thermal stress conditions.

\subsubsection{Statistical Description of Fracture Sets: Tools to De- scribe Fracture Geometries and Effects on Permeability}

To constrain the location of fluid flow pathways and associated permeability, fracture systems need to be evaluated in three dimensions (3-D) and at the various scales at which they occur Bonnet et al., 2001; Berkowitz, 2002). Fracture systems commonly comprise several fracture sets which can result from different geological events, and have 
a)

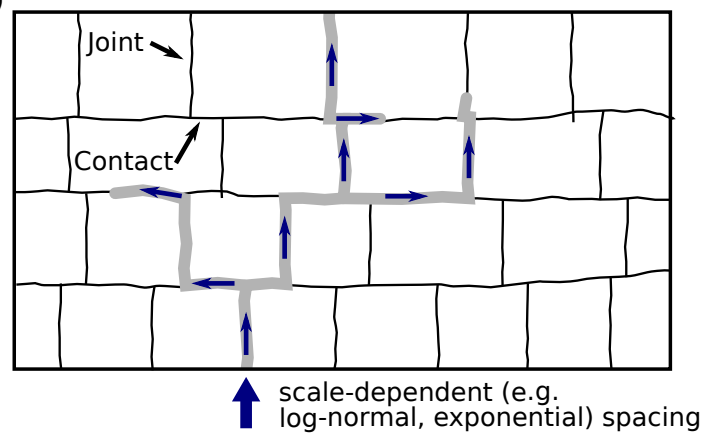

b)

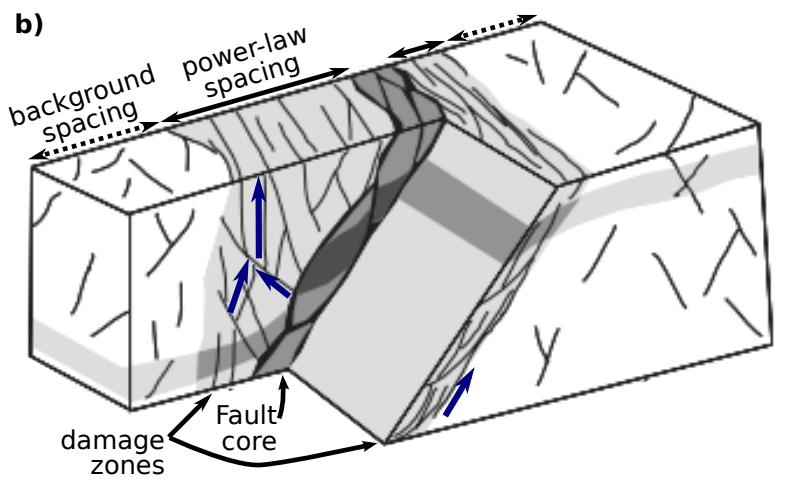

Figure 1.2: Schematic fracture and fluid flow models. a) In layered jointed rocks, flow can occur along joints and contacts between layers; the layer thickness and ratio of layer tensile to interface shear strength (Schöpfer et al., 2011) are characteristic scales that affect the joint spacing (after Gudmundsson et al., 2002). b) In fault damage zones, fracture spacing is clustered near faults (commonly power-law), and a background spacing (power-law or scale-dependent) prevails away from the fault zone (after Manzocchi et al., 2010).

different impacts on permeability (Priest, 1993, Dezayes et al., 2015). Each fracture set can be described in terms of its orientation, and the probability distribution of fracture attributes: aperture (for open fractures), thickness (for veins), spacing, density and length (Priest, 1993). Fracture attributes have been shown to variously follow power-law, exponential, gamma, log-normal and power-exponential distributions (see Bonnet et al., 2001; Torabi and Berg, 2011, and references therein). The probability distribution form of fracture attributes varies as a function of the processes that control fracture generation and propagation (Bonnet et al., 2001; André-Mayer and Sausse, 2007). In layered systems, stratabound joint spacing is usually best approximated by regular, log-normal, gamma, or exponential distributions, with characteristic sizes linked to the layer thickness and ratio of the layer tensile to interface shear strength (Gillespie et al., 1993; Rives et al., 1992; Schöpfer et al., 2011). Fluids tend to flow along these joints and contacts between layers (Figure 1.2a). In contrast, the spacing of fractures close to faults, and length of non-stratabound fractures are commonly well approximated by power-law distributions which are scale-independent and argued to be intrinsic to fracture growth mechanisms (Figure 1.2b; see Bonnet et al. (2001) for review; McCaffrey et al. (2003); Davy et al. (2006, 2013); Johri et al. (2014)), although this may partially reflect sampling bias and the mixing of different fracture sets (Gillespie et al., 1993; Nicol et al., 1996).

In carbonate layered systems, both scale-dependent and scale-independent fracture spacing have been observed for different fracture sets in the same outcrop, and associated with layering and faulting, respectively (Agosta et al., 2010). The fracture sets of different scaling were also associated with different permeability roles: storage for the fracture sets of the layered host rock, and support for fluid migration for the fractures 


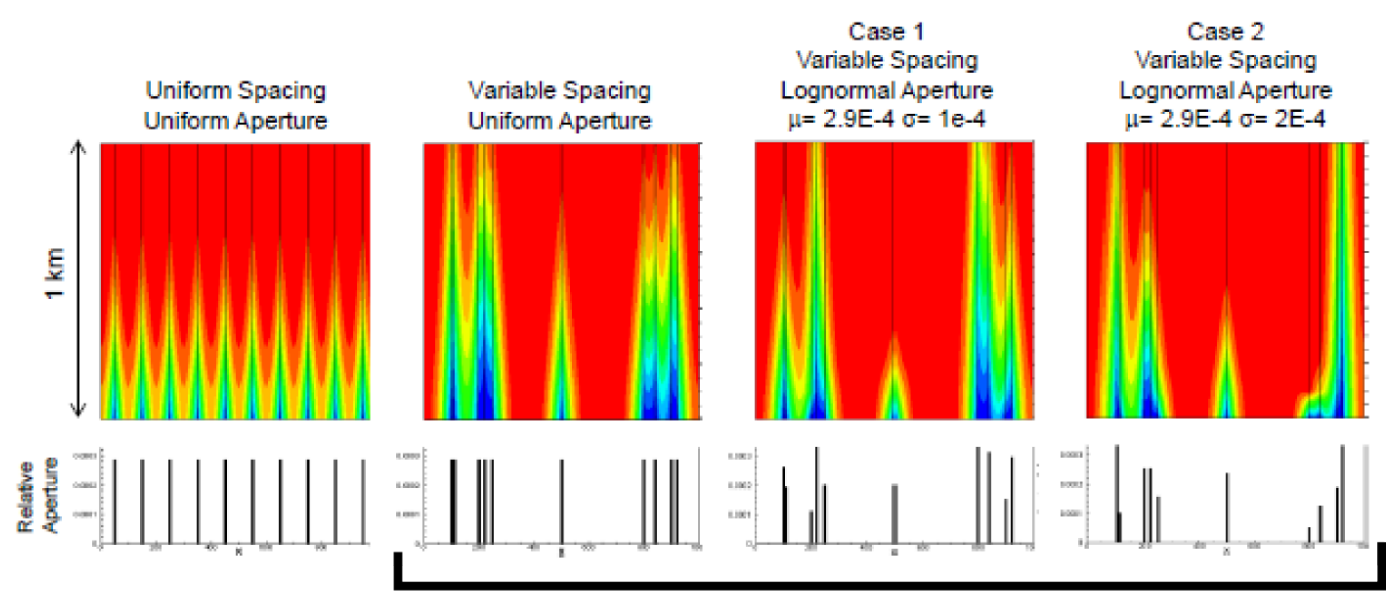

Non-uniform distributions: faster thermal breakthrough

Figure 1.3: Visualisation of temperature distributions for parallel fracture cases with initial rock temperature of $200^{\circ} \mathrm{C}$ and inlet water temperature of $75^{\circ} \mathrm{C}$, for variable fracture spacing and aperture (Doe et al. 2014$)$. The bar chart at the bottom of each block has the location and aperture of the fracture immediately above.

of the fault-damage zones (Agosta et al., 2010). The type of scaling law also directly affects the fluid flow patterns and heat extraction efficiency, as presented by numerical fluid and heat flow simulations performed for enhanced geothermal systems evaluations (Figure 1.3, Doe et al. 2014). Indeed, clustering of fracture locations and presence of wide aperture fractures channelise flow and focus heat exchanges to specific regions of the rock mass, rather than homogeneously sweeping the whole domain. Non-uniform spacing and aperture distributions result in a quicker thermal breakthrough compared to a uniform spacing and aperture. Thus, power-law spacing distribution would result in a higher degree of channelisation than exponential (which are usually less clustered) or log-normal (which have a minimum spacing threshold) distributions.

The statistical representation of fracture systems is thus critical for revealing the controls on the fracture system generation, and for numerically modeling fracture system geometries. The probability distribution of fracture geometrical attributes must be studied for different fracture sets (Priest, 1993), as each set may have been formed under different processes, and may affect permeability differently Agosta et al. 2010. Dezayes et al., 2015). Measurements obtained from borehole image logs (as used in this thesis) are subject to specific sampling biases which need to be taken into account Rider, 1996; Massiot et al., 2015) and have not to date been fully described, either for the delineation of fracture sets, or the evaluation of the scaling laws.

The classic method for delineating fracture sets is to plot fracture orientations on hemispherical projections (stereonets) and contour the projected poles to fracture planes by their areal density, yielding a "Fisher" density. "Kamb contouring" is another classic stereonet contouring method which measures the deviation from a random distribution $(K a m b, 1959)$. Kamb's method is less influenced by the sample size than 
Fisher's Vollmer, 1995). Manually delineating fracture sets using contours is an intuitive and straightforward method to apply, thanks to stereonet software packages. However, the delineation of fracture sets from these contours is subjective and may vary between data analysts. Cluster analysis of fracture orientation provides transparent means of separating fracture orientation data into sets, as proposed by Shanley and Mahtab (1976). Agglomerative, K-means, and fuzzy K-means clustering have been used to analyse orientation data derived from manual scanline measurements, predominantly for engineering purposes (e.g., Hammah and Curran, 1998, 1999, Zhou and Maerz, 2002, Tokhmechi et al., 2011; Li et al., 2014b), but not fully explored for borehole log-derived datasets.

The probability distribution form of fracture attributes is commonly estimated by least-square regressions and comparison of the coefficients of correlation. In particular, power-law exponents are commonly estimated using linear regression to the cumulative density function (CDF) of observations in doubly logarithmic axes ("log-log" plots; see McCaffrey et al. (2003) for example). However, this method is not mathematically robust, and does not unambiguously demonstrate that the observations are power-law (Clauset et al. 2009). The maximum likelihood estimation (MLE) method is preferred to least-square fitting for estimating which probability distribution and associated parameters best fit a set of data (Laslett, 1982, Villaescusa and Brown, 1992, Clauset et al., 2009). The MLE method estimates values for the parameters of a given distribution that maximises a likelihood function, i.e. finds the parameter set that makes the observed data most likely. While MLE methods are commonly used by statisticians, they are more rarely used in geoscientific applications.

\subsubsection{Fracture Models}

Numerical methods developed to represent fracture system geometries and associated preferential flow pathways at reservoir scale can be grouped in three main categories (Neuman, 2005):

1. Discrete Fracture Network (DFN) models where fracture planes are explicitly represented (Dershowitz and Einstein, 1988; de Dreuzy et al., 2013)

2. Continuum models with equivalent permeability associated to a representative element volume (e.g. Pollyea et al., 2014). A refinement of such method includes dual-porosity models where matrix and fractures are modelled as two different elements, and can represent the exchanges of fluid, heat or chemical species between the fracture and matrix elements (e.g., Pruess, 1992)

3. A combination of the two previous methods with hybrid models of a non-uniform continuum containing a small number of discrete dominant features (e.g., Lee et al., 2001; Hernandez et al., 2015) 
In this work, we will focus on DFN models, and discuss other relevant modeling strategies in Chapter 6. DFNs represent a fractured rock mass explicitly by combining an ensemble of statistical laws describing the fracture size, orientation, transmissivity and density, in a stochastic framework (e.g., Davy et al., 2006). The probability density function form and parameters of the fracture geometrical properties are determined from field observations, typically on outcrops, wells and tunnels de Dreuzy et al., 2013, and references therein). Common assumptions of fracture length and density rely on the assumptions that object properties are power-law, yielding a fractal system, i.e. without intrinsic length scale (e.g., Bour and Davy, 1997). Fractal systems can thus accommodate the occurrence of fractures at all scales. In the case of layered formations, such power-law distributions do not necessarily represent well these systems, an issue which has not been extensively studied for volcanic and metamorphic rocks.

Validating numerical fracture models relies on the observations of fracture geometries and reservoir-scale permeability data. Probability distributions of fracture aperture and linear density can be obtained at depth from borehole data, including cores and wireline logs (Barton and Zoback, 1992, Genter and Traineau, 1996). To varying degrees, borehole data can constrain in-situ stress, and petrophysical and hydrological conditions at depth, which control fracture geometries and associated fluid flow Evans et al., 2005; Zoback, 2007, and references therein), but are limited to the vicinity of the borehole and are typically sparse. Conversely, distributions of length and fracture intensity (combining density and length) rely mostly on analogue outcrop studies (Priest, 1993; Mauldon et al., 2001; Torabi and Berg, 2011) and stereological calculations Wang, 2005). However, the volumetric fracture density (equivalent to a fracture porosity if fractures are open), critical for constraining fluid flow numerical models, is the most difficult parameter to evaluate given the lack of true 3-D outcrop observations and the limited resolution of geophysical surveys. The combination of outcrop analogue and borehole data is thus a typical strategy to generate geologically-informed fracture models, which can then be constrained by production data (e.g., Hodgetts, 2013). Such combined studies are rare in volcanic and metamorphic rock settings.

\subsection{Geological Settings of the Three Case Studies}

In this thesis, quantitative analysis and description of fracture systems has been conducted across the New Zealand plate boundary zone (Figure 1.4), primarily using data from the Ruapehu volcano, Rotokawa Geothermal Field, and the metamorphic hangingwall of the central Alpine Fault. Results from the Ruapehu volcano provide information on the initial state of the rock mass prior to burial and faulting of the andesitic formations hosting the Rotokawa Geothermal Field. Findings from the metamorphic hangingwall focus on fault- and foliation-related fractures, and complement 
the results from the Rotokawa Geothermal Field in a differently layered host rock.

\subsubsection{New Zealand Plate Boundary}

New Zealand is located at the convergent boundary between the Pacific and Australian plates. The south-westward motion of the Pacific plate relative to the Australian plate is accommodated differently throughout New Zealand, from the west-dipping Hikurangi subduction margin off the east coast of the North Island (Reyners et al., 2006), to the oblique continental collision in the South Island principally accommodated along the Alpine Fault, a $460 \mathrm{~km}$-long southeast-dipping transform fault (Figure 1.4 Norris and Cooper, 2001). Offshore to the southwest of the South Island, the Australian plate subducts eastwards beneath the Pacific plate along the Puysegur margin.

In the North Island, extension occurs within the Taupo Volcanic Zone (TVZ) at $\sim 7$ to $15 \mathrm{~mm} / \mathrm{yr}$ Darby et al., 2000; Wallace et al., 2004), and is associated with high heat flow (average heat flux of $700 \mathrm{~mW} / \mathrm{m}^{2}$; Bibby et al., 1995), active volcanism (Wilson et al. 1995, and geothermal systems (Wilson and Rowland, 2016, and references therein). The TVZ is $\sim 350 \mathrm{~km}$ long and up to $\sim 60 \mathrm{~km}$ wide, and extends from Ruapehu volcano to the Bay of Plenty (Figure 1.4). A series of NE-SW-trending normal faults in the central TVZ define the Taupo Rift (Wallace et al., 2004; Nicol et al., 2007). These normal faults are laterally discontinuous and typically dip 60-80 within the shallow crust ( $\sim 3 \mathrm{~km} ;$ Rowland and Sibson, 2001; Villamor and Berryman, 2001, 2006; Rowland et al., 2010, Seebeck et al., 2010).

\subsubsection{Ruapehu Volcano}

Ruapehu is New Zealand's largest active volcano, located at the southern end of the TVZ, $\sim 100 \mathrm{~km}$ south of the Rotokawa Geothermal Field (Figure 1.4; Gamble et al., 2003; Conway et al., 2016). This volcano has been built via a series of intense constructional events separated by periods of erosion, sector collapse and low-level activity (Hackett and Houghton, 1989; Price et al., 2012). Ruapehu lavas are mostly weakly vesicular (5\% average), strongly porphyritic andesites (35-55\% phenocrysts), accompanied by rare basaltic andesites, dacites and basalts (Price et al., 2012; Conway et al., 2016).

\subsubsection{Rotokawa Geothermal Field}

The Rotokawa Geothermal Field, located $10 \mathrm{~km}$ north of Taupo (Figure 1.4), is a high-temperature geothermal reservoir with a $174 \mathrm{MW}$ installed electricity production capacity (Hernandez et al. 2015$)$. The Rotokawa deep reservoir is hosted in >1.89 Myrold (Eastwood et al., 2013; Chambefort et al., 2014) 800-2100 m-thick andesite lavas and breccias (Browne et al. 1992; McNamara et al., 2016a). Hydrothermally-altered 


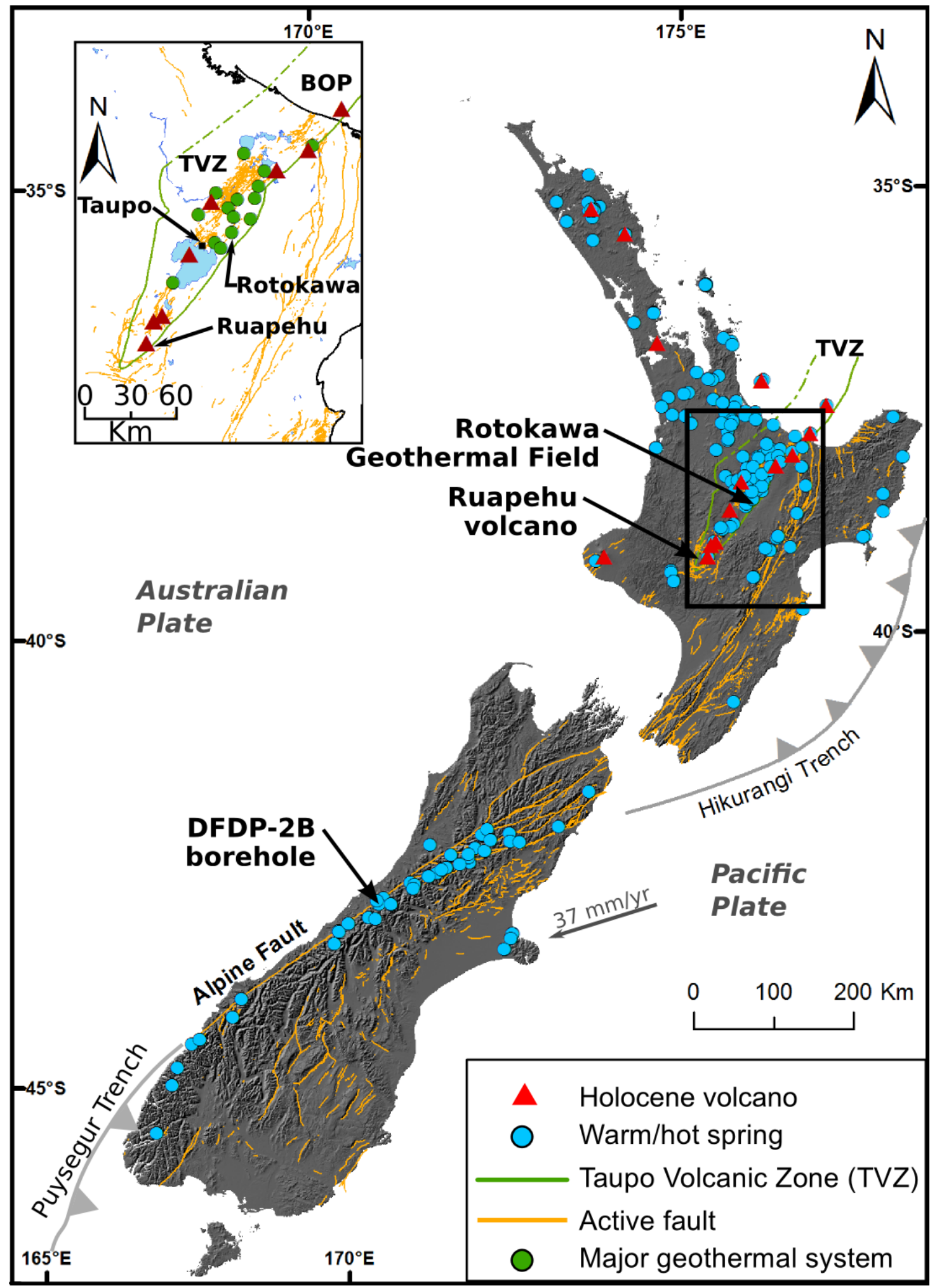

Figure 1.4: Locations of the three sites studied in this thesis: Rotokawa Geothermal Field, Ruapehu volcano and DFDP-2B drillhole in the Whataroa Valley. The map also displays New Zealand plate boundaries, the distribution of thermal springs (Mongillo and Clelland, 1984; Reyes et al., 2010), active volcanoes, mapped active faults in New Zealand (http://data.gns.cri.nz/af/), and the relative plate motion (DeMets et al., 1994). Inset: major high-temperature systems that have been delineated by geophysics and/or drilling. TVZ: Taupo Volcanic Zone; BOP: Bay of Plenty. 
andesite lavas are porphyritic with 51-62 wt. \% $\mathrm{SiO}_{2}$ Andersen, 2011; Siratovich et al., 2015). In the Rotokawa deep reservoir, fluid flow is mostly controlled by fractures, and the permeability distribution is heterogeneous and compartmentalised (Hernandez et al. 2015; McNamara et al., 2015, 2016a). As in many of the geothermal fields in the TVZ, sustainably maximising the energy production from the Rotokawa Geothermal Field requires an understanding of fluid flow, and how it is affected by fractures.

Textures and mineralogy of the andesite lavas and breccias at the Rotokawa and nearby Ngatamariki Geothermal Fields are consistent with them being products of a complex composite volcano such as Ruapehu, with the main edifice forming the Rotokawa Andesite (Chambefort et al., 2014; McNamara et al., 2016a). Andesite flows at Ruapehu are thus chemically and morphologically appropriate analogues for the Rotokawa andesites prior to their burial and deformation during rifting.

\subsubsection{Central Alpine Fault, Deep Fault Drilling Project (DFDP)}

The Alpine Fault, able to generate large (moment magnitude $(\mathrm{Mw})>7$ ), and possibly great $(\mathrm{Mw}>8)$ earthquakes, is a dextral transpressive fault late in its interseismic cycle (Berryman et al. 2012). The geometry, rheology and hydrological conditions of the Alpine Fault are being studied as part of the Deep Fault Drilling Project (DFDP) by drilling boreholes, defining lithological and petrophysical properties of the rock, and installing permanent geophysical equipment (Townend et al., 2009). Two boreholes (100 and $152 \mathrm{~m}$ deep) drilled at Gaunt Creek (central Southern Alps) during the first phase of the DFDP programme sampled fault rocks within $70 \mathrm{~m}$ of the Alpine Fault (Sutherland et al., 2012). Lithological (Toy et al., 2015), structural (Williams et al., 2016), physical (Boulton et al., 2012), and hydraulic (Carpenter et al., 2014) properties measured from cores revealed an intensely fractured damage zone and impermeable fault core, while wireline logging analysis and hydraulic measurements provided details of the structure and petrophysical properties of a geochemically distinct alteration zone (Sutherland et al., 2012; Townend et al., 2013).

As part of the second phase of the DFDP project, the DFDP-2B borehole was drilled in late 2014 down to $818 \mathrm{~m}$ below the ground surface in the Whataroa valley, central Southern Alps, in the hangingwall of the Alpine Fault (Figure 1.4 Sutherland et al., 2015). An extensive wireline logging programme offers the first opportunity to study the sub-surface structural and petrophysical characteristics of the hangingwall of the Alpine Fault, along a $630 \mathrm{~m}$-long section of the borehole increasingly close to the Alpine Fault with depth. The bedrock sampled by the geophysical borehole logs is composed predominantly of ductilely sheared mylonite-series lithologies exhumed from deep within the Alpine Fault zone. The protolith is interpreted as amphibolite facies metasediments classified as part of the Aspiring Lithologic Association of the Torlesse Composite Terrane. 


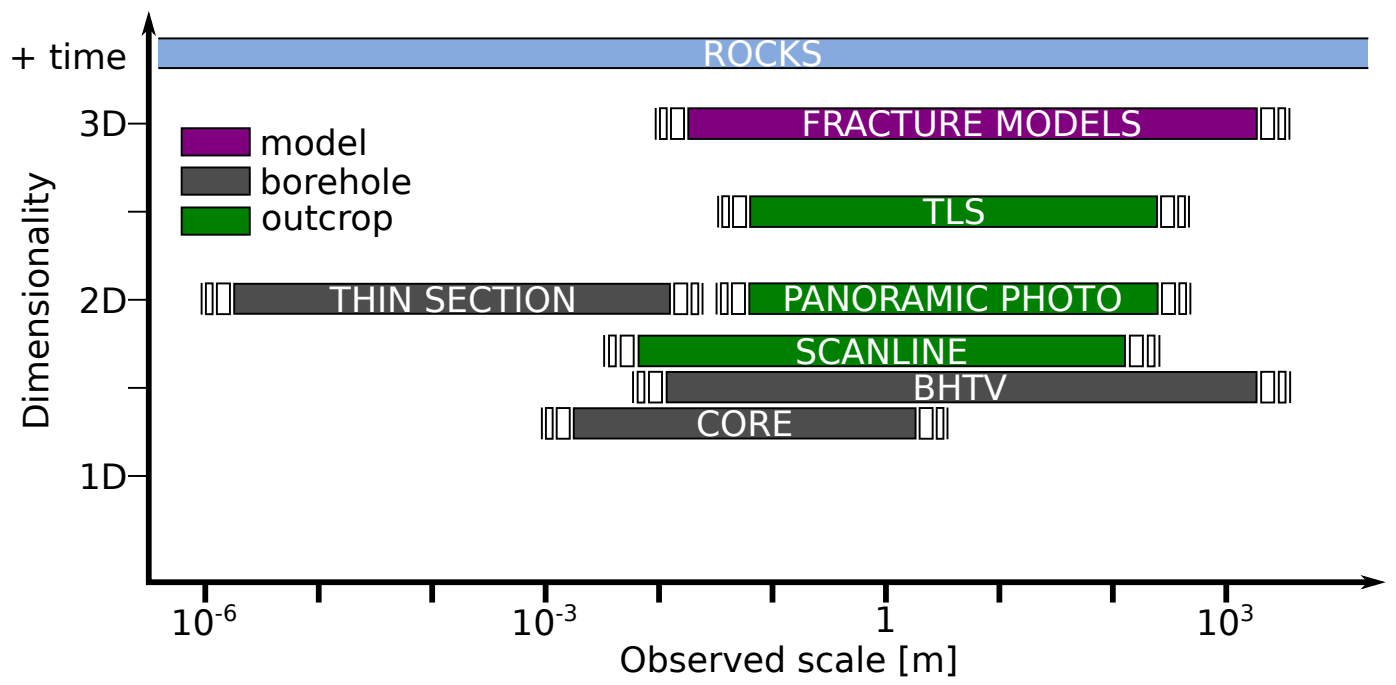

Figure 1.5: Scale, dimensionality and type (outcrop/borehole/model) of sampling methods used in this thesis. The left-hand side of each box represent the minimum scale of observed fractures, while the right-hand scale relates to the maximum size of outcrop, borehole or model. Fractures occur naturally in rocks at all scales, and vary spatially and temporally. TLS: terrestrial laser scanner; BHTV: acoustic borehole televiewer log; thin section observed by optical microscopy.

\subsection{Data}

Several types of data covering various scales and dimensions (D) are described in this thesis (Figures 1.5 and 1.6). Outcrop data comprise manual scanlines, measured along a 1-D line but with fracture length measurements away from the scanline (thus 1.5-D); 2-D panoramic photographs; and TLS data which is principally 2-D but captures 3-D variations of outcrop faces. In addition, petrophysical data measured on Ruapehu rock samples are presented in Appendix A. Borehole data comprise 2-D thin-sections (using findings reported by Siratovich et al., 2014), cores, and BHTV logs. BHTV logs are principally 1-D but are extended to 1.5-D at scales smaller than the borehole diameter because of the cylindrical sampling. Individual datasets are affected by their own sampling biases, which are addressed specifically in each case. The geometrical parameters derived from the combination of datasets can be employed to constrain 3-D fracture models (Figure 1.6 $\mathrm{g}$ ), which can then be used to model fluid pathways spanning $10^{-2}-10^{3}-\mathrm{m}$ scales Chilès, 2005; Kissling et al., 2015b; Aprilina et al., 2015).

\subsubsection{Outcrop Datasets}

Scanline measurements are a classical method of evaluating fracture attributes from outcrops Priest, 1993). Fracture location (providing spacing and density measurements), orientation, length and connectivity with nearby fractures are measured manually along a tape measure (Figure 1.6a). These detailed measurements are invaluable 
a) MANUAL SCANLINE, PANORAMIC PHOTOGRAPH
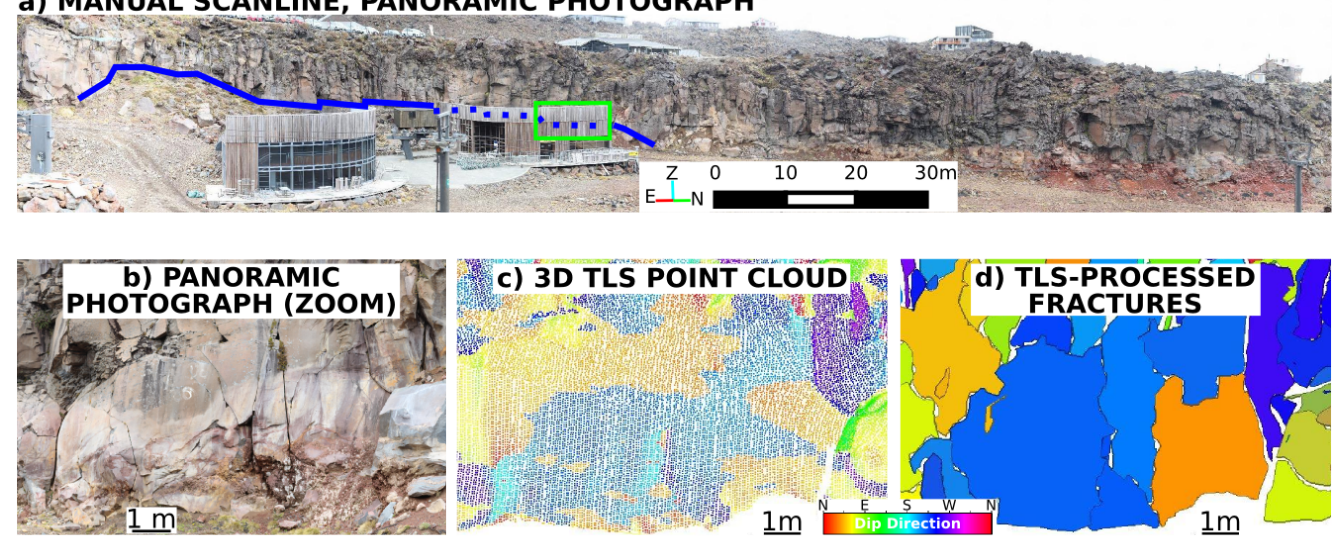

e) CORES

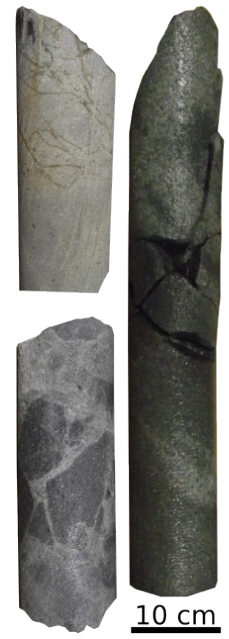

f) BHTV LOGS

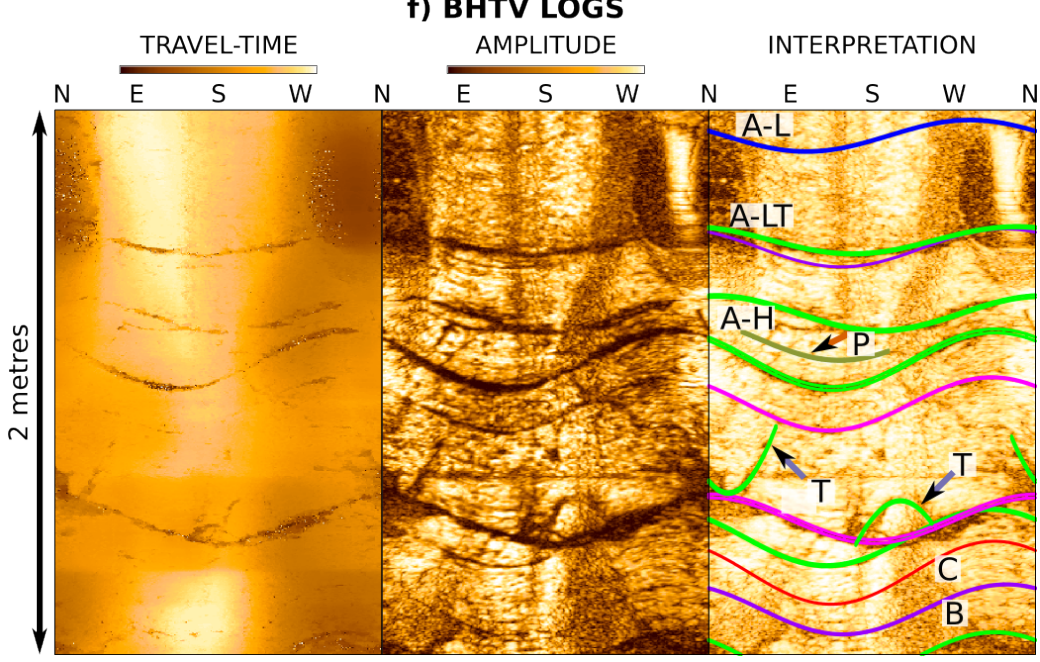

g) FRACTURE MODELS

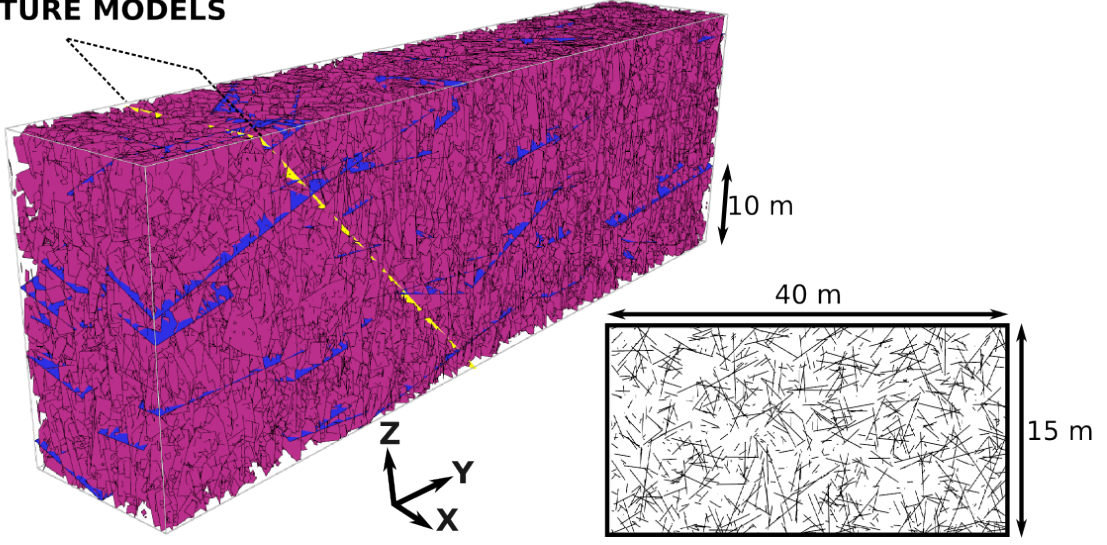

Figure 1.6: Data used in this thesis. a) Outcrop data: manual scanline (blue line) and high-resolution panoramic photograph. TLS dataset was acquired over the entire displayed outcrop (Chapter 2). b-d are enlargements of the area behind the building outlined by the green square. b) High-resolution panoramic photograph (taken from a different angle from a)). c) 3-D TLS point cloud, coloured by the local dip direction. d) Fracture planes fitted to the TLS point cloud. e) Cores from the Rotokawa Geothermal Field (Chapter 4). f) BHTV log travel-time and amplitude unwrapped images, and fracture interpretation. Different line and arrow colours relate to different morphological characteristics (Chapter 5). g) Discrete Fracture Network (DFN) model of a lava-flow-scale model (Chapter 2). A trace map crossing the DFN (yellow and dotted lines) samples traces of fractures intersecting the virtual plane. 
for gaining insights into the fracture development processes, and calibrating other fracture measurements. However, scanlines are 1.5-D at best, time-consuming to acquire, and limited by the accessibility of the rock face.

TLS is a technique increasingly used in geosciences (Abellan et al., 2016), and generates dense 3-D point clouds (usually millions of points) by measuring the return time of a laser pulse emitted at small angular increments (Figure 1.6c; Pfeifer and Briese, 2007; Buckley et al., 2008). High-resolution panoramic photographs provide visual details in addition to the shape of the outcrop captured by the TLS point cloud (Figure 1.6 a-b). TLS datasets allow the delineation of fracture geometries over large outcrops when fractures form faces with 3-D relief (Figure 1.6c-d). Fracture analysis from TLS datasets has been used for slope stability studies (Deparis et al., 2008), fracture density characterisation in sedimentary and plutonic formations (Jones et al., 2008; Rotevatn et al. 2009; Pearce et al., 2011), input for DFN models (Wilson et al., 2011) and reservoir simulations (Enge et al., 2007; Fabuel-Perez et al., 2010), particularly in the hydrocarbon industry (e.g., Hodgetts, 2013, and references therein). The large fracture datasets obtained from TLS sometimes contradict empirical relationships defined from scanlines alone, and can be more appropriate for reservoir analogue studies Pearce et al. 2011). TLS has been applied to volcanological settings in order to monitor eruptions (James et al., 2009, Massey et al., 2010; Slatcher et al., 2015), reconstruct volcanic facies in 3-D (Nelson et al., 2011; Geyer et al., 2015), characterise lava flow roughness (Mazzarini et al., 2008), delineate units of a lava flow sequence (Nelson et al., 2015), and evaluate dyke swarm geometries (Ni et al., 2016). The characterisation of fracture networks in volcanic rocks from TLS has been used to evaluate slope stability in rhyolitic ignimbrite Agliardi et al. 2013) and as an analogue for basalt lava-flowhosted reservoirs in $\mathrm{CO}_{2}$ sequestration projects (Pollyea and Fairley, 2012). To date, however, TLS has not been used to study fracture system geometries in andesite lavas.

\subsubsection{Borehole Datasets}

Cores provide material for geological, geochemical, petrophysical and structural studies of subsurface rocks (e.g., Genter and Traineau, 1996, Chambefort et al., 2014), on hand-size specimens or thin sections (Figure 1.6p). However, cores are rarely oriented and usually do not provide fracture orientation data. In addition, cores are typically sparsely acquired after the initial geothermal field exploration phase, and recovery rates can be variable, especially in fractured reservoirs. The location and depth of core sampling is motivated by geological questions, but also by drilling schedules and economic considerations, and often focuses on reservoir rocks. All of these constraints typically limit cores to 1-10- m-long "spot" cores distributed within a large rock volume (e.g., Chambefort et al., 2014).

BHTV logs provide oriented images of the inside of borehole walls along sections 
up to several kilometres in length, using ultrasonic signals emitted and received by the logging tool that reflect off the borehole wall (Zemanek et al., 1970, Poppelreiter et al. 2010, and references therein). The return-time of the acoustic signal provides an estimate of the borehole diameter in all directions, assuming an appropriate speed of sound in the borehole fluid (Wagner and Kretzschmar, 2007, Massiot et al., 2015). In addition, the amplitude of the returned signal relates to the acoustic properties of the borehole wall, dominated by the borehole wall roughness and mineral composition (Davatzes and Hickman, 2010). Fractures intersected by the boreholes that have sufficient acoustic impedance contrast with the host rock can be detected on BHTV logs, together with their orientation and apparent thickness (Figure 1.6 $)$ ). The resulting fracture sampling along the borehole is intermediate between a continuous, oriented cylindrical core, and an outcrop scanline.

While BHTV logs have lower resolution than cores, ( 10-50 mm versus 1-50 mmthick fractures, respectively), they provide continuous measurements along the borehole which are crucial in the absence (e.g., for DFDP-2B) or scarcity (e.g., for the Rotokawa Geothermal Field) of cores.

\subsection{Thesis Content}

Each of the chapters in this thesis represent self-contained studies. They were prepared as manuscripts for journal publication (excluding this chapter and a synthesis chapter), contain an abstract and introduction and, as such, can be read in isolation. Preparing the chapters as a series of papers results in some duplication within the thesis, particularly in the beginning of each chapter.

The research described in this thesis has been conducted by me (Cécile Massiot), unless stated otherwise. However, as each chapter is in an advanced stage of preparation for publication, they have been written in the first person plural ("we"), as is common practice for publications involving more than one author. In particular, Christopher E. Conway and Timothy A. Little contributed insights into the geological contexts of the Ruapehu volcano (Chapter 2) and Alpine Fault (Chapter 5), respectively. Chapter 5 also benefited from collaborations during and since DFDP-2B drilling. The first person plural writing style also reflects the fact that my supervisors have provided guidance and advice throughout the project.

This thesis is separated into the following chapters:

Chapter 2: Quantitative Geometric Description of Fracture Systems in an Andesite Lava Flow Using Terrestrial Laser Scanner Data

This chapter is a study of fracture geometries in a young $(\sim 6 \mathrm{ka}$, Conway et al., 2016), outcropping andesitic lava flow on the flanks of the Ruapehu volcano. The chap- 
ter incorporates outcrop measurements (TLS, calibrated with scanline and panoramic photographs), fracture analysis, and DFN fracture model generation. Fractures are extracted from the shape of the TLS point cloud using the recently developed Facets plugin of the CloudCompare software (Dewez et al., 2016, CloudCompare, 2016). Fracture analysis reveals a highly connected fracture system within the blocky interior of the lava flow, partially connected to heterogeneous breccias. DFN models generated from the measured fracture orientations, length and areal densities yield a highly connected fracture system similar to outcrop observations. This outcrop study provides information on the initial fracture system of the Rotokawa andesite reservoir studied in Chapters 3 and 4 , prior to its burial, faulting and hydrothermal alteration history. In

addition, the results are applicable to shallow groundwater reservoir evaluation $\overline{C u s-}$ todio, 2007) and volcanic hazards (Houghton et al., 1987; Voight, 2000).

This chapter is in the final stages of preparation for publication in the Journal of Volcanology and Geothermal Research.

\section{Chapter 3: Statistical Methods of Fracture Characterisation Based on Acoustic Borehole Televiewer Log Analysis}

Chapter 3 details methods for analysing fracture attributes (orientation, thickness and spacing) measured on BHTV logs. These methods include clustering of fracture orientations, and maximum likelihood estimations of fracture thickness and spacing applied to truncated distributions. Statistical criteria rank a selection of probability distributions by how well they describe the scaling properties of fracture attributes. This method is also applicable to other fracture attributes such as length or throw. This chapter uses BHTV log data acquired at the Rotokawa Geothermal Field, which are further analysed in Chapter 4. Analysis includes consideration of sampling bias, a strategy to accommodate the varying BHTV log quality, and an estimation of the number of data points required to unequivocally select a single probability distribution form representing the dataset amongst the tested selection of models.

\section{Chapter 4: Tectonic, Lithologic and Thermal Controls on Fracture System Geometries in an Andesitic High-Temperature Geothermal Field}

Chapter 4 presents the results of the statistical analysis of the orientation, thickness, spacing and volumetric densities of fractures in the Rotokawa andesite geothermal reservoir. Fracture analysis at three scales (BHTV logs, cores, and thin section measurements, the latter previously reported by Siratovich et al., 2014), suggest that fracture location and density are controlled by lithologies such as possible stratification within the andesite sequence, and faulting. By contrast, fracture thickness and volumetric density display scale-independent behaviours at core- and BHTV-log-scales which may extend to larger structures, with orientations largely controlled by tectonic stresses. We assess the potential roles of stress and connectivity on fracture permeabil- 
ity, and propose avenues for developing reservoir-scale fracture models, which would help refine controls on permeability in this reservoir.

Chapters 3 and 4 are in the final stages of preparation for publication in the Journal of Geophysical Research.

\section{Chapter 5: The Alpine Fault Hangingwall Viewed From Within: Structural Analysis of Acoustic Image Logs in the DFDP-2B Borehole, New Zealand}

This chapter evaluates foliation and fracture orientations and morphologies in the metamorphic Alpine Fault hangingwall, using BHTV log interpretation of the DFDP-2B borehole. We interpret the foliation planes and fractures identified on BHTV logs in the context of existing outcrop studies of the Alpine Fault hangingwall, and discuss the implications of this fracture system for potential fluid flow pathways.

This chapter is part of a series of studies arising from the DFDP-2B drilling that took place late in 2014, and is in the final stages of preparation for publication in the Geological Society of America Bulletin. Results of this chapter are currently being used in several studies aimed at characterising fluid flow circulations near the Alpine Fault, and implications on stress and thermal aspects which may control earthquake nucleation and propagation.

\section{Chapter 6: Synthesis}

The final chapter summarises the key results of the thesis, and provides practical avenues to develop geologically-informed fracture models in lava sequences, and near the Alpine Fault. Suggestions for further research on what controls fracture permeability are also outlined.

Several appendices are included in the thesis, in either print or electronic form.

\section{Appendix A: Petrophysical Tests of Samples From Iwikau Village, Ruapehu Volcano}

Density, porosity, Vp, Vs and unconfined compressive strength (UCS) were measured at the University of Canterbury laboratory on 13 core plugs from rock samples at the Happy Valley lava flow (studied in Chapter 2) and nearby Meads Wall dyke, Ruapehu volcano. The mechanical anisotropy shown by the UCS test results of the Happy Valley provides a mechanistic explanation of the fracture development in the lava flow, which can be used to interpret rock masses that do not have the benefit of such detailed fracture mapping. These tests will be integrated into a publication in preparation for the New Zealand Journal of Geology and Geophysics aimed at refining the mechanical controls on fracture propagation and connectivity in andesite rocks. 


\section{Electronic appendices}

Two electronic appendices containing the results of BHTV log interpretation in the Rotokawa Geothermal Field (Chapter 4) and the DFDP-2B borehole (Chapter 5) are included on a CD. 


\section{Quantitative Geometric Description of Fracture Systems in an Andesite Lava Flow Using Terrestrial Laser Scanner Data}

\section{Abstract}

Permeability hosted in andesitic lava flows is dominantly controlled by fracture systems, with geometries which are often poorly constrained. This chapter explores the fracture system geometry of an andesitic lava flow formed during its emplacement and cooling over gentle paleo-topography, on the active Ruapehu volcano, New Zealand. The fracture system comprises column-forming and platy fractures within the blocky interior of the lava flow, bounded by autobreccias partially observed at the base and top of the outcrop. We use a terrestrial laser scanner (TLS) dataset to extract columnforming fractures directly from the shape of the point cloud over an outcrop area of $\sim 3090 \mathrm{~m}^{2}$. Fracture processing is validated using manual scanlines and high-resolution panoramic photographs. Column-forming fractures are either steeply or gently dipping with no preferred strike orientation. Geometric analysis of fractures derived from the TLS, in combination with virtual scanlines and trace maps, reveals that: (1) steeply dipping column-forming fracture lengths follow a scale-dependent exponential or lognormal distribution rather than a scale-independent power-law; (2) fracture intensities (combining density and size) vary throughout the blocky zone but have similar mean values up and along the outcrop; and (3) the areal fracture intensity is higher in the autobreccia than in the blocky zone. The inter-connected fracture network has a connected porosity of $\sim 0.5 \%$ that would promote fluid flow vertically and laterally within the blocky zone, and is partially connected to the autobreccias. Depending on burial and alteration history, autobreccias may act either as lateral permeability connections, or as barriers in reservoirs. A discrete fracture network model generated from these geometrical parameters yields a highly connected fracture network, consistent with outcrop observations. 


\subsection{Introduction}

Numerous geothermal, groundwater, $\mathrm{CO}_{2}$ storage, radioactive storage, and more recently hydrocarbons reservoirs are hosted in lava flows Custodio, 2007; Feng, 2008; Bertani, 2016). In these reservoirs, fluid flow is inferred to be controlled by fractures and faults, but the identification and geometric description of permeable fracture networks often remains problematic Arnórsson, 1995; Chen et al., 2001; Nemčok et al. 2007).

Fracture morphologies in lava flows have been extensively studied in basalts (e.g., DeGraff and Aydin, 1987; Schaefer and Kattenhorn, 2004; Hetényi et al., 2011; VyeBrown et al., 2013) and less frequently in andesites (Doyuran et al., 1993; Deng et al., 1995; Mc Caffrey et al., 2003; Spörli and Rowland, 2006; Conway et al., 2015). Lava flows commonly have massive and fractured interiors, surrounded by upper and lower autobreccias caused by mechanical breakage (Bonnichsen and Kauffman, 1987). The most prominent fracture types in the flow interior are column-forming joints, generated by the thermal stresses associated with cooling of the lava flow from the margins to the interior by contact with water or atmosphere (DeGraff and Aydin, 1987, 1993; Lore et al. 2000). Column-forming fractures propagate orthogonal to the maximum thermal gradient (DeGraff and Aydin, 1987, Lore et al., 2001). The cooling front is approximately elliptical, which causes the column-forming fractures to be orthogonal to the edges of the flow. The commonly observed increase in the spacing of those fractures towards the interior of the flow reflects a slower cooling rate Schaefer and Kattenhorn, 2004).

Fracture systems in lava flow-hosted reservoirs result from the initial emplacement of the lava as well as subsequent interaction between tectonic, thermal, and hydrothermal processes (Fournier, 1998; Gudmundsson et al., 2001, Nemčok et al., 2004; Hetényi et al., 2011). The Rotokawa geothermal reservoir located in the Taupo Volcanic Zone (TVZ), New Zealand, is an example of one such andesite-hosted geothermal reservoir, in which the hydrothermally altered andesites have a low porosity (4.4-16.3\%) and high strength (unconfined compressive strength -UCS- of 70-211 MPa), and fluid flow is structurally controlled (Siratovich et al., 2014; McNamara et al., 2015). Although the importance of fractures for fluid flow in this geothermal field is widely accepted, the fractures themselves are poorly understood. Establishing a "baseline" for the fracture distribution formed during the lava flow emplacement is a critical step to improving the targeting of permeable fractures in reservoirs.

Fracture models can help with the management of resources via analysis of preferential flow pathways. Discrete fracture network (DFN) models describe fluid flow processes through rock masses by stochastically generating fractures Dershowitz and Einstein, 1988; de Dreuzy et al., 2013). DFNs rely on the input of fracture geometries 
(mainly orientation, size, frequency and aperture). A number of probability distributions of fracture size (length or radius) and density have been used to generate fracture network models in basaltic and andesitic reservoirs (Doyuran et al., 1993; Deng et al., 1995; Chen et al., 2001; Lee et al., 2010; Pollyea et al., 2014). Fracture geometries depend on multiple environmental and lithological parameters including composition, weathering, cooling rate, morphology, paleo-topography, and crystal size Grossenbacher and McDuffie, 1995; Karpuz and Paşamehmetoğlu, 1997; Custodio, 2007; Kattenhorn and Schaefer, 2008; Hetényi et al., 2011). Among other factors, the higher silica content of andesites (57-63 wt \% $\mathrm{SiO}_{2}$ ) than basalts $\left(45-57 \mathrm{wt} \% \mathrm{SiO}_{2}\right.$ ), associated with different cooling rates, may produce different fracture distributions Forbes et al., 2014). The large range of silica content in andesites may also create variability in the fracture systems between different andesite lava flows.

Probability distributions of fracture geometries are typically estimated from 1-D scanlines in outcrop (Priest, 1993) and boreholes using image logs (e.g., Barton et al., 1995; Massiot et al. 2015), or 2-D circular sampling in outcrops (Mauldon et al. 2001). Terrestrial laser scanner (TLS) datasets, also called terrestrial Lidar, allow a comprehensive fracture analysis of an entire outcrop in 3-D, which is therefore less subject to observation biases than scanlines or window samplings of limited sizes (though other specific biases have to be considered; Lato et al., 2010), and supports more reliable statistics on fracture geometries (Hodgetts, 2013). The large fracture datasets obtained from TLS are sometimes different from empirical relationships defined from scanlines, and TLS data may be more appropriate than scanlines for reservoir analogue studies (Pearce et al. 2011). TLS studies are increasingly applied to geoscientific questions (Abellan et al., 2016), including in volcanic settings (e.g., Massey et al., 2010; Nelson et al., 2011; Geyer et al., 2015; Nelson et al., 2015). Fracture delineation from TLS has been used for slope stability analysis (Deparis et al., 2008), fracture density statistics in sedimentary and plutonic formations (Jones et al., 2008; Rotevatn et al., 2009; Pearce et al., 2011), and input into reservoir simulations Enge et al., 2007; FabuelPerez et al., 2010) or DFN models (Wilson et al., 2011) in the hydrocarbon industry (see Hodgetts, 2013, and references therein). Fracture systems within lava flows have rarely been studied using TLS, focusing on basalt (Pollyea and Fairley, 2012) rather than andesite flows.

Several approaches have been developed to extract fractures and their properties from a TLS point cloud, using various computational strategies and degrees of user interaction. These approaches aim to delineate fracture traces perpendicular to, or planes sub-parallel to exposures. Fracture traces or faces can be delineated manually on photographs before (Nelson et al. 2011 ) or after draping over the point cloud (called digital outcrop model (DOM); Buckley et al., 2008; Hardebol and Bertotti, 2013). The generation of a DOM requires the point cloud to be meshed, which can be a computationally complex task where there is significant outcrop topography. Methods 
for automatically sampling fracture traces have been developed (Umili et al. 2013 ), but the fracture orientations in such cases are not well constrained. Fully- or semiautomatic detection of fracture faces typically depends on the a-priori definition of fracture sets of specific orientations, and operate with or without meshing of the point cloud (e.g., Olariu et al., 2008; García-Sellés et al., 2011; Gigli and Casagli, 2011; Riquelme et al., 2014). The ability of these techniques to detect fractures is limited in the absence of fracture populations of specific orientations, as is the case in lava flows. Pollyea and Fairley (2011) used the higher roughness occurring at the fracture edges to detect fracture densities, but this technique does not provide fracture orientation and size.

In this paper, we aim to provide constraints on DFN models of the fracture system in an andesitic lava flow of the Ruapehu volcano, New Zealand, based on the analysis of a TLS dataset. We test the applicability of the recently developed "Facets" methodology for extracting fractures from a TLS point cloud based solely on the shape of the outcrop (Dewez et al., 2016). We discuss the implications of the fracture geometries obtained from the TLS dataset for the permeability of andesite-hosted reservoirs, using synthetic DFN models.

\subsection{Geological Setting}

\subsubsection{Ruapehu volcano, Central North Island of New Zealand}

Ruapehu is New Zealand's largest active andesitic volcano, located at the southern end of the Taupo Volcanic Zone (TVZ) in the central North Island, New Zealand (Figure 2.1, Hackett and Houghton, 1989; Wilson et al., 1995). Ruapehu may be as old as $340 \mathrm{kyr}$ (Tost and Cronin, 2015), but the exposed edifice was mainly constructed by basaltic-andesite to dacite lava effusion in the last $200 \mathrm{kyr}$ Gamble et al. 2003, Conway et al., 2016). Ruapehu is primarily composed of blocky lava flows and autobreccias, with 54-65 wt. \% $\mathrm{SiO}_{2}$ (Hackett and Houghton, 1989). Porphyritic andesite lavas of the Whakapapa Formation were emplaced between 10 and 4 ka and form much of the upper flanks of the volcano (Conway et al., 2015, 2016). Post-glacial lava flows such as those of the Whakapapa Formation have low height-to-width ratios $(<1: 10)$, are typically $<5 \mathrm{~m}$ thick, and are associated with autobreccias and welded spatter (Conway et al., 2015).

\subsubsection{Description of the Happy Valley Outcrop}

The present study focuses on a lava flow in "Happy Valley" located near the Iwikau Village ski field on the NNW flank of Ruapehu (Figure 2.17). The Happy Valley outcrop is a $\sim 210$ m-long section of a 4.5-21 m-thick, young ( 6 ka; Conway et al., 2016) 

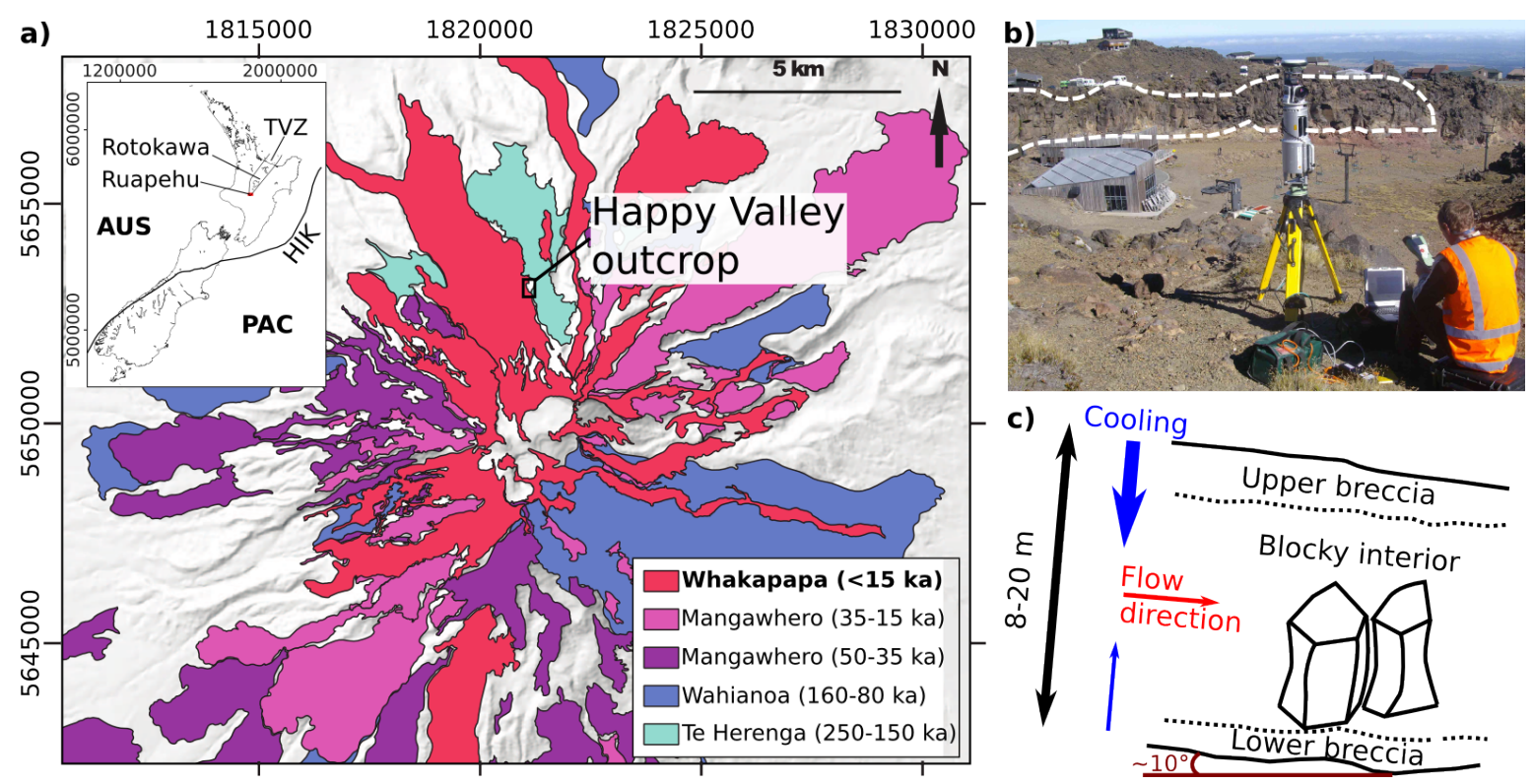

Figure 2.1: Location of the "Happy Valley" lava flow outcrop. a) Simplified geological map of Ruapehu Volcano with major formation distribution and ages (after Conway et al., 2016). The Happy Valley outcrop is shown by the black box. Locations of Ruapehu volcano at the southern tip of the Taupo Volcanic Zone (TVZ) is shown by the inset figure. The Hikurangi Trough (HIK) marks the seafloor expression of westward subduction of the Pacific plate (PAC) beneath the Australian plate (AUS). Extent of the geologic map of Ruapehu is shown by the red dot on inset map. b) TLS acquisition; the outcrop studied is delimited by the dashed line. c) Schematic crosssection of the outcrop, a 8-20 m-thick blocky lava flow emplaced over a gently dipping paleo-slope $\left(\sim 10^{\circ}\right)$. The upper and lower breccias displayed here are observable only in parts of the outcrop. 
lava flow of the Whakapapa Formation with 59 wt. \% $\mathrm{SiO}_{2}$ (Wysoczanski, 1989). The rock face predominantly presents the interior of a blocky andesite lava flow emplaced over low-angle topography (Figures 2.1 b-c, 2.5a). The lava flow is underlain by a lower autobreccia (referred to as "breccia" in the remaining of the paper) which is intermittently observed. The upper breccia is mostly hidden by vegetation or eroded, and a 1-3 m-thick zone is observable in the northern part of the outcrop (Figure 2.9). The results presented in this study are representative of this "simple" configuration and do not take into account fracturing caused by lava-ice interactions (Conway et al., 2015), emplacement over a knob, variations in composition, faulting, or other parameters described by Hetényi et al. (2011).

Fractures studied in this outcrop are joints formed in mode I during the emplacement and cooling of the lava flow at the ground surface (DeGraff and Aydin, 1987; Pollard and Aydin, 1988), and are referred to as "fractures" in the general sense of a discontinuity. Two main types of fractures are observed in the Happy Valley outcrop:

1. Column-forming fractures: most common fractures, which delimit columns of polygonal cross-section roughly perpendicular to the top and base of the lava flow. These fractures are straight to curved (Figure 2.2a), and form blocky breakage.

2. Platy fractures: closely-spaced fractures, spatially clustered, sub-parallel to each other, along which the lava breaks into flaky sheets or smaller platy fragments (mm-cm-thick, 1-100 cm across; Figure 2.2 ) as described by Lescinsky and Fink (2000) and Conway et al. (2015). Platy fractures are generally subhorizontal in the central zones of the flow and moderately to steeply inclined in the upper zones. They form along planes of weakness parallel to aligned crystals, and thin, vesicle-rich layers (Conway et al., 2015).

\subsection{TLS Processing, Fracture Processing and Fracture Analysis}

\subsubsection{Data Acquisition}

TLS devices emit and receive laser pulses at predefined vertical and horizontal angular intervals. The resulting high-density 3-D point cloud is a measurement of the location $(\mathrm{x}, \mathrm{y}, \mathrm{z})$ and backscattered energy of each reflected pulse (Pfeifer and Briese, 2007; Buckley et al., 2008). TLS scans were acquired using the Riegl LMS-Z420I instrument from three positions precisely located by GPS measurements, with one station mounted directly on the TLS and two additional stations located on top of the outcrop. The acquisition of TLS scans from several locations limits blind areas that the laser rays cannot directly access behind the two buildings, and reduces the line-of-sight and 

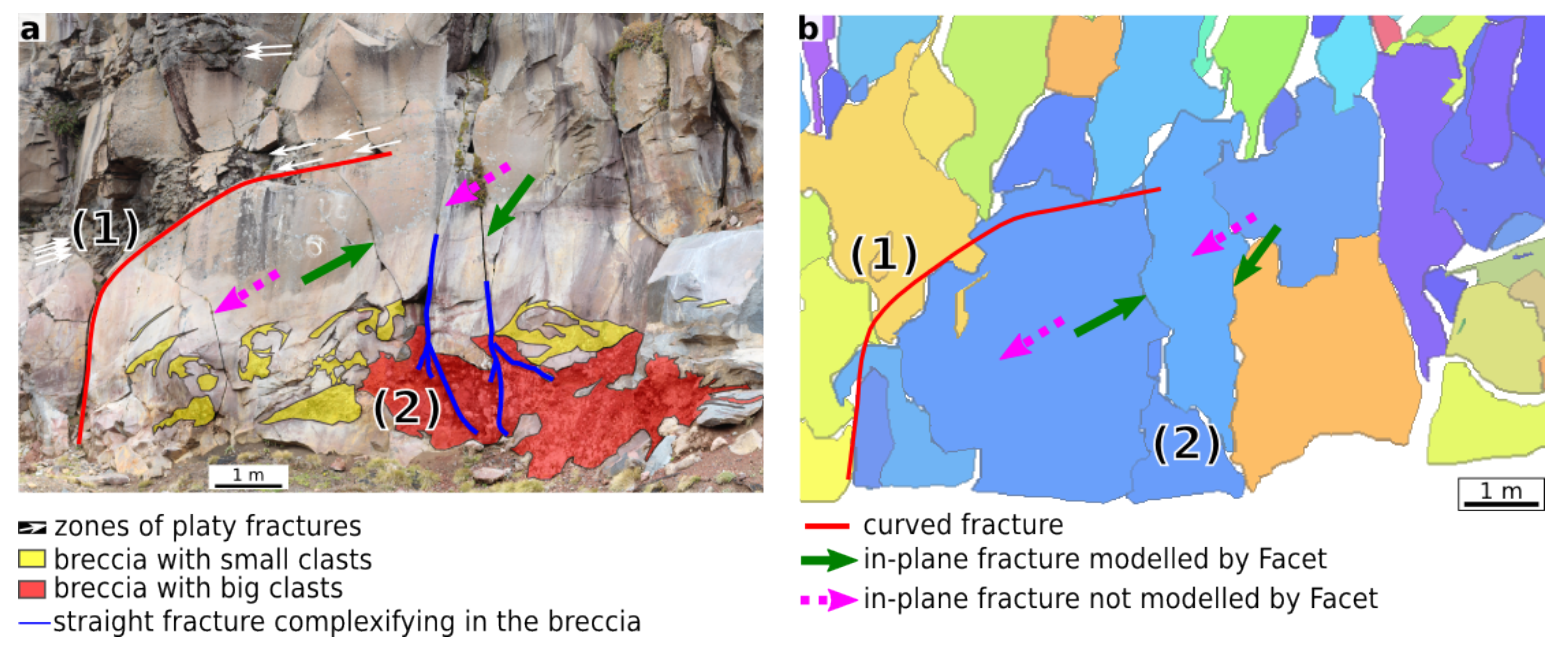

Figure 2.2: Detection limits of the TLS and factors influencing the column-forming fracture propagation. a) Section of the panoramic photograph displayed in Figure 2.5 $\mathrm{a}$. b) Fracture planes processed from the TLS point cloud with the Facets algorithm. Note the fitting of a single plane for the area of dense platy fractures (1); the absence of detection of breccia in the TLS (2); the limited detection of in-plane column-forming fractures (purple arrows); and the bifurcations of fractures at the interface between the blocky interior and the brecciated zones. An enlargeable version of the photograph presented in a) is available at: http: //www. gigapan. com/gigapans/191973.

distance bias (Buckley et al., 2008). TLS point cloud resolution is $\sim 4-5 \mathrm{~cm}$ after processing (Figure 2.3. Appendix 2.A). Georeferenced photographs were also taken from each TLS location. While photographs can be visualised in 3-D after being draped over the TLS point cloud, they lack the resolution to show fine details $(<1 \mathrm{~cm})$.

Three high-resolution panoramic photographs were acquired with a Gigapan system from different angles to cover the entire outcrop studied, providing details at the centimetric to millimetric scale (Gigapan, 2016, Figures 2.2 and 2.5a). As they are not georeferenced, Gigapan photographs cannot be draped over the point cloud, but their higher resolution complements the georeferenced pictures.

The location, length, and terminating relationships of 192 column-forming fractures were manually measured along a $100 \mathrm{~m}$-long scanline near the ground level (Figure 2.5a, Table 2.1). Changes of facies (blocky or breccia) were also noted. The scanline is made at different heights near the base of the outcrop, and with variable trend and plunge, which ensures sampling of fractures at a range of angles to the flow direction. In addition to the scanline per se, the orientation and spacing of two zones of closelyspaced platy fractures intersecting, and located near the scanline, respectively, were measured. While the scanline does not cover the whole area measured by the TLS, it offers detailed insights into the relationships between fractures, and a calibration for the TLS fracture delineation. 


\subsubsection{Fracture delineation From the TLS Point Cloud}

Fractures are extracted from the TLS dataset based solely on the shape of the 3-D point cloud, using the Facets plugin in CloudCompare (Dewez et al., 2016, CloudCompare, 2016), as detailed in Appendix 2.A and Figure 2.3. Fractures are defined by portions of the point cloud that are approximately coplanar (Figure 2.4 a). The resulting fracture dataset contains the geometry of the planes forming the outcrop: the XYZ coordinates on the centre of each plane in the geographic coordinate system, together with the orientation and size (length, height, surface) of the locally fitted plane. Overall, 3403 fractures covering an area of $\sim 3090 \mathrm{~m}^{2}$ were delineated from the TLS point cloud.

Fracture orientations extracted from the TLS, and measured by hand, only differ by a few degrees in both dip magnitude and dip direction (Appendix 2.A), a discrepancy consistent with the accuracy of TLS reported by Sturzenegger and Stead (2009a) and of hand-held compass measurements.

\subsubsection{Sampling Bias}

Both the scanline measurements and the fracture dataset extracted from the TLS are affected by the under-sampling of the smallest fractures and the low probability of intersection of the scarce larger fractures with the outcrop face Pickering et al. 1995 . Priest, 2004).

On the scanline, fractures $<0.05 \mathrm{~m}$-long were not recorded. The length was fully measured when the terminations were $\sim 3.5 \mathrm{~m}$ above, and $0.5-1.5 \mathrm{~m}$ below the scanline (depending on the height of the scanline above ground). The longest fracture $(11.6 \mathrm{~m})$ is sub-horizontal and was not affected by the sampling bias. Fracture length where one or both terminations were beyond the scanline swath are referred to as censored, and are thus longer than the reported measurement (Pickering et al., 1995).

The TLS-processed dataset contains only column-forming fractures. The detection of long fractures by the TLS is only affected by the height of the outcrop and location in line-of-sight (Buckley et al., 2008). The fracture processing extracts planes bounding the point subsets (Figure 2.4 b), which limits the bias related to the measurement of chords to fractures instead of the fracture length itself (f-bias in Priest, 2004). Fractures with length $<0.2 \mathrm{~m}$ were not resolved from the TLS point cloud (Appendix 2.A). The centimetre-scale relief of column-forming fracture's growth increments visible on panoramic photographs is too small to be captured by the TLS (Appendix 2.A. Figure 2.A.1). Similarly, in-plane fractures that do not exhibit any 3-D relief are not resolved (Figures 2.2, 2.A.1). Changes of facies (blocky lava and breccia), are also not detected by the TLS processing (Figure 2.2). The platy fractures are small and non-planar: they are not resolved by the TLS and are fitted collectively by a single plane (Figure 2.2. 


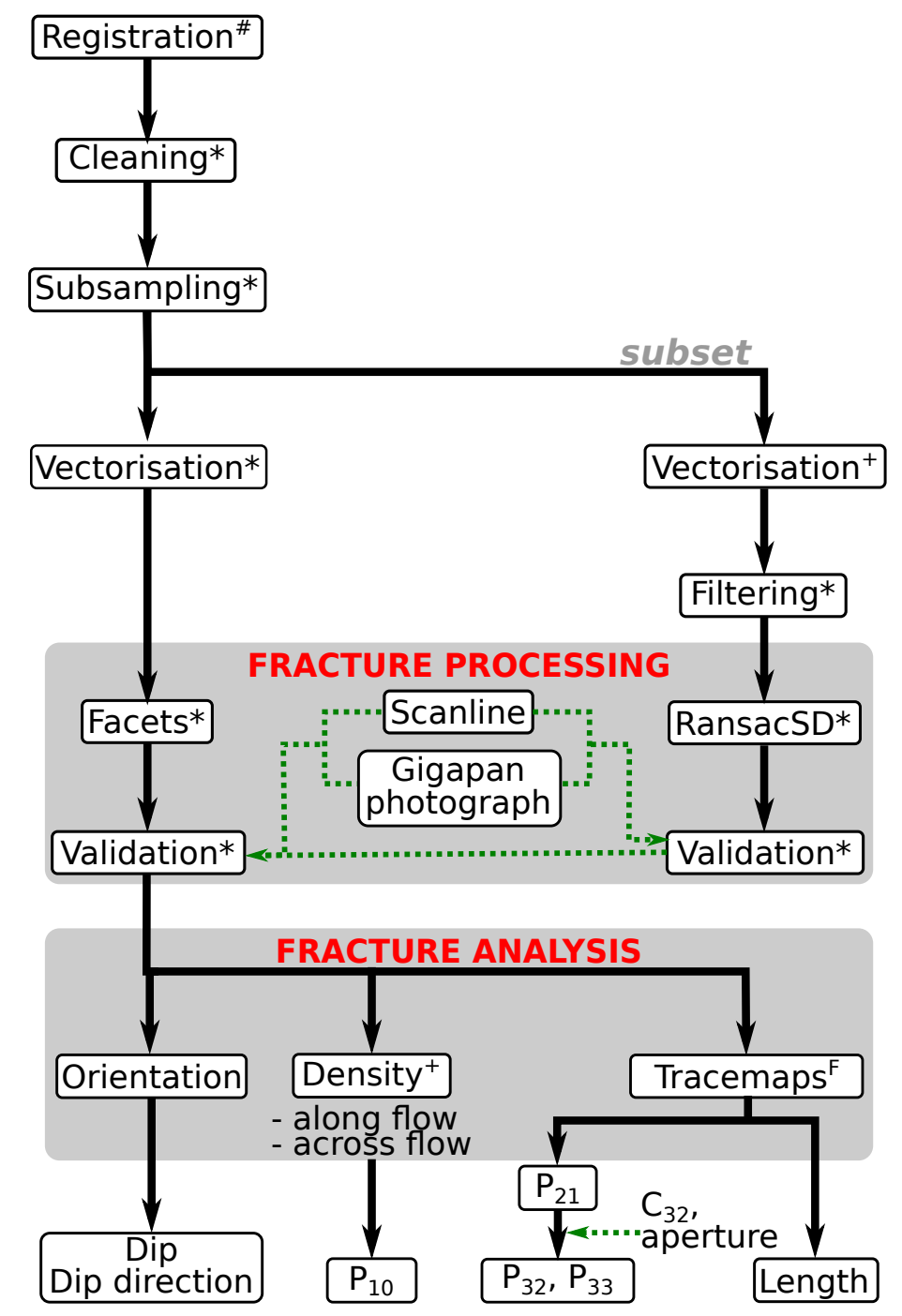

Figure 2.3: Flowchart of the TLS data processing and fracture analysis using a combination of Riegl RiscanPro (\#), SEFL (+), CloudCompare v2.7.0 (*) with the RansacSD (Schnabel et al. , 2007) and Facets plugin (Dewez et al., 2016), and Fracman ( ${ }^{\mathrm{F}}$ ) software. Note that $\mathrm{P}_{i j}$ refers to the dimensionality of the sampling region (i) and the dimensionality of the fracture property $(\mathrm{j})$. 

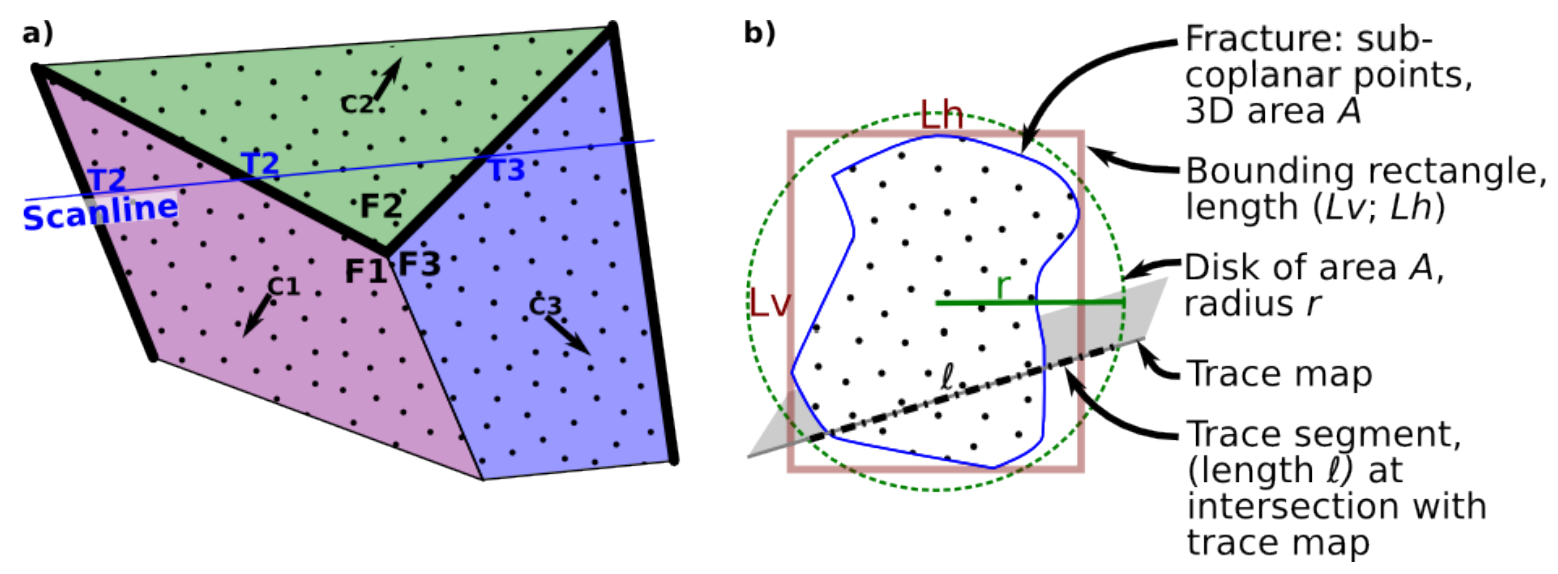

Figure 2.4: Fracture measurements on scanline, TLS-processed fractures, and trace maps. a) Schematic of the fracture sampling of three fractures (F1, F2 and F3): the scanline measures fractures at the intersection between the scanline and the fracture (T1, T2 and T3) while the TLS processing extracts the centre (C1, C2 and C3) of each fracture, the orientation (characterised by the normal to planes, arrows) and the size (3-D area $A$, lengths $L h$ and $L v$ ). b) The bounding rectangle of the point subset of each fracture has length $L h$ and $L_{V}$ in horizontal and vertical directions. The trace length $\ell$ is measured at the intersection between the trace map and the disk of radius $r$ and area $A$.

\subsubsection{Fracture Analysis Method}

The inputs to discrete fracture network (DFN) models are probability distributions of fracture orientations, lengths, intensities (which combine fracture density and size), terminations, and apertures (Berkowitz, 2002). The steps used in this study to analyse fractures are summarised in the lower part of Figure 2.3 .

Fracture orientation is analysed directly from the results of the TLS processing and scanline measurements (Figure 2.5-d). The spatial distribution of fracture area is presented in Figure 2.5e. To assess the variability of fracture density within the outcrop, we evaluate $\mathrm{P}_{10}$ (the number of fractures intersected per unit length of scanline; Dershowitz and Herda, 1992) along a series of virtual scanlines through the TLS-processed dataset at $10 \mathrm{~cm}$ intervals in two orthogonal directions. Sub-horizontal scanlines parallel to the brecciated base (dipping $\sim 10^{\circ}$ ) measure $\mathrm{P}_{10}$ from the base to the top of the lava flow, whereas sub-vertical scanlines perpendicular to the brecciated base (dipping $\sim 84^{\circ}$ ) measure $\mathrm{P}_{10}$ upflow to downflow. The calculations are made with the "Stratigraphy" tool of SEFL software (Laubach et al., 2009; García-Sellés et al., 2011). $\mathrm{P}_{10}$ values are affected by the shape of the outcrop, so we present results for zones which are sufficiently wide, high, and planar, namely zones 2, 4, 6, and 8 illustrated in Figure 2.7. We include in the analysis only those virtual scanlines that (1) are $\geq 10 \mathrm{~m}$ long, (2) intersect $\geq 10$ fractures, and (3) are fully contained within the extent of the outcrop.

The $\mathrm{P}_{32}$ parameter (which expresses the summed area of fracture per volume of rock) is a measure of fracture intensity that combines both fracture density and size. 


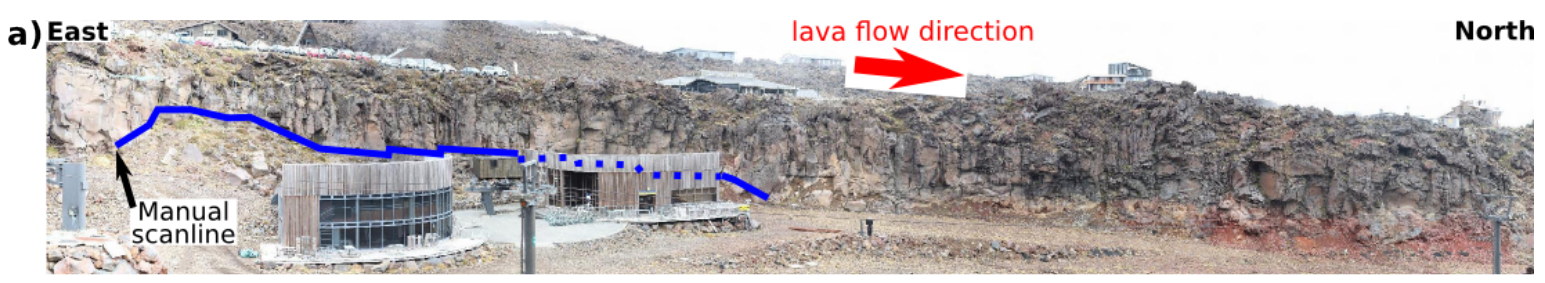

b)
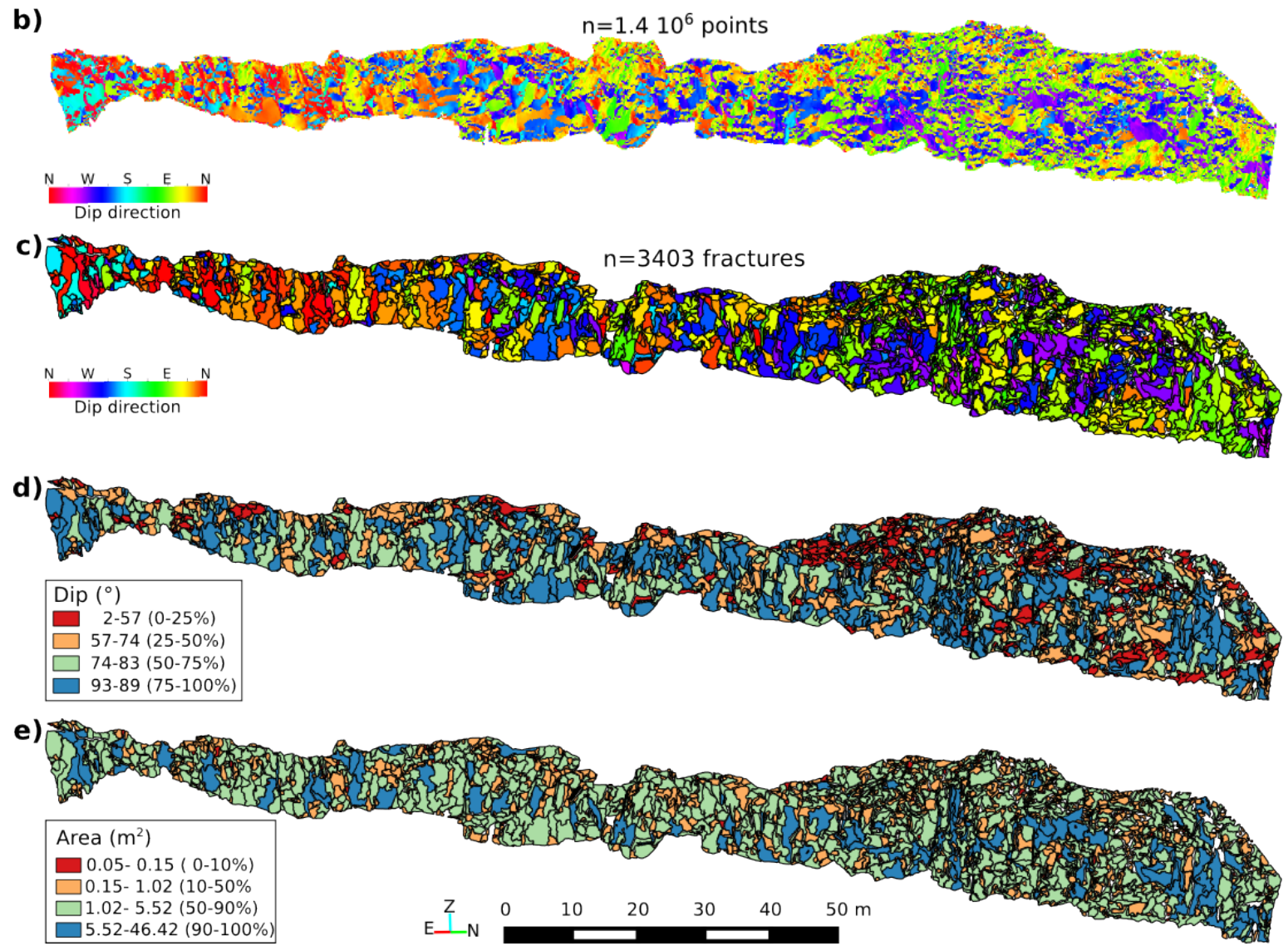

Figure 2.5: Fracture delineation of the Happy Valley terrestrial laser scanner (TLS) point cloud. a) Gigapan panoramic photograph composed of 430 individual photographs with the trace of the manual scanline (solid/dashed blue line) and the lava flow direction. b) TLS point cloud used for the fracture delineation and resampled every $4 \mathrm{~cm}$, coloured by the local dip direction. c) Fractures delineated using the Facets plugin, coloured by dip direction, d) dip magnitude, and e) area. Note the non-regular ranges for the dip magnitude and area scales; the percentage of fractures falling into each category is indicated in brackets. c-e: 3-D fractures projected onto a $N W$-SE striking, vertical plane. Each colour patch represents a single fracture. 
$\mathrm{P}_{32}$ is a favoured intensity measure with which to characterise fracture systems, as it is invariant with respect to the distribution of fracture size and is scale independent; that is, it does not depend on the volume studied or the orientation of the measurements (Dershowitz and Einstein, 1988). $\mathrm{P}_{32}$ cannot be directly measured but can be derived from the $\mathrm{P}_{21}$ parameter (the summed length of fracture traces per area of rock):

$$
P_{21}=L_{f} / A_{t}
$$

where $\mathrm{L}_{f}$ is the total length of fracture traces measured on a surface of area $\mathrm{A}_{t} \cdot \mathrm{P}_{21}$ is classically measured on trace maps, which represent the intersection ("traces") of fractures with an outcrop (Figure 2.4 b; see examples in Figure 2.13b). In this work, $\mathrm{P}_{21}$ is evaluated in two ways: (1) directly using the TLS-processed fractures as the area used to sample fracture traces (although not perfectly planar); and (2) using virtual trace maps in the Fracman software. The first case assumes that TLS-processed fractures are tiled next to each other over a surface best-fitting the outcrop of area $A_{t}$ :

$$
P_{21}=\frac{\sum_{i}^{n} p_{i} / 2}{A_{t}}
$$

with $p_{i}$ being the perimeter of each fracture. The edge of each fracture is shared with a neighbouring fracture, so the total length of the segments is half the summed perimeter of each fracture. $A_{t}$ is measured using Gocad software by fitting a surface to the point cloud.

In the second case, two series of virtual sections orthogonal to the outcrop face are generated, yielding virtual trace maps parallel and orthogonal to the lower breccia (i.e. sub-horizontal and sub-vertical). In this case, the $\mathrm{A}_{t}$ is measured as

$$
A_{t}=l_{\text {scanline }} \cdot W
$$

with $l_{\text {scanline }}$ the length of the polyline formed at the the intersection between the outcrop surface and the trace map plane, and $W$ a constant, fixed at $1 \mathrm{~m}$ based on visual inspections, which takes into account the sampling area of the shape of the outcrop.

Finally, the $\mathrm{P}_{32}$ parameter is proportional to $\mathrm{P}_{21}$ :

$$
P_{32}=C_{32} P_{21}
$$

where $\mathrm{C}_{32}$ is related to the orientation distribution of the fractures (Wang, 2005). When all fractures have the same aperture, $\mathrm{P}_{33}$, the volume of fractures per volume of rock (and thus the fracture porosity when reported as a percentage), is proportional to the fracture aperture a: 


$$
P_{33}=a \cdot P_{32}=a \cdot C_{32} \cdot P_{21}
$$

The probability distribution of fracture lengths is evaluated from the virtual trace maps parallel and orthogonal to the lower breccia. Fracture traces sampled at the intersections of the TLS-processed fractures and the trace maps are "chords" to the actual fracture extent (Figure 2.4b). Fractures are modelled as penny-shaped disks, with a radius $r$ and an aperture a. Probability distributions are fitted to the fracture traces, and then converted to a distribution of fracture radii in Fracman following Zhang et al. (2002). Three distributions commonly found to describe fracture lengths are tested (Bonnet et al. 2001): exponential, log-normal and power-law (Table 2.1). Fitting of these probability distributions to empirical cumulative density functions (CDFs) of fracture segments is evaluated with Kolmogorov-Smirnov (K-S) tests. A good fit is characterised by a low distance D between the estimated and empirical CDFs, and a high significance level. Finally, the fit of the complementary cumulative density function (CCDF; which is 1-CDF) to a linear model in log-linear and log-log plots, provides additional indication of the preferential fit to an exponential or a power-law distribution, respectively.

\subsection{Results: Fracture Geometries in the Happy Valley Outcrop}

\subsubsection{Orientation}

Column-forming fractures have two main orientations: steeply dipping $\left(>55^{\circ}\right)$, and gently dipping $\left(<30^{\circ}\right)$, without a preferred strike orientation (Table 2.1). The scanline measurements reveal these two dip magnitude sets (Figure 2.6a) and the large variety of strike orientations (Figure 2.7b), regardless of the scanline trend which varies along the profile. The ratio of gently to steeply dipping fractures is 1:3, after correction for orientation sampling bias (Table 2.1; Terzaghi, 1965). Platy fractures observed on scanlines are gently dipping $\left(10-25^{\circ}\right)$.

TLS-processed column-forming fractures are steeply dipping and strike sub-parallel to the outcrop, regardless of its orientation (Figure 2.7a), which confirms that there is no preferred strike orientation. Gently-dipping column-forming fractures are not well delineated by TLS processing because they are usually not in direct line-of-sight, and have insufficient 3-D relief (Figure 2.6 a). The overall high density of fractures striking $\mathrm{N}-\mathrm{S}$ relates to the large outcrop area with $\mathrm{a} \sim 174^{\circ}-354^{\circ}$ strike orientation (zones 1 to 4). The varied strike orientation of the Happy Valley outcrop makes it suitable for extracting reliable statistics, similar to making scanlines in several directions as recom- 
Table 2.1: Geometrical parameters of column-forming fractures measured from scanline and TLS-processed datasets, and parameters used to model DFN models (Figure 2.13). Length is presented as minimum/median/maximum values. $n$ : number of fractures per set.

\begin{tabular}{|c|c|c|c|}
\hline \multirow[t]{2}{*}{ Parameter } & \multirow[t]{2}{*}{ Data } & \multicolumn{2}{|c|}{ Column-forming fracture set } \\
\hline & & $\begin{array}{cc}\text { steeply dipping } & \text { intermediate } \\
\left(\text { dip }>55^{\circ}\right) & \left(\text { dip } 30-55^{\circ}\right)\end{array}$ & $\begin{array}{l}\text { gently dipping } \\
\left(\operatorname{dip}<30^{\circ}\right)\end{array}$ \\
\hline \multirow[t]{2}{*}{$\mathrm{n}$} & scanline & $149\left(335^{*}\right)$ & $25\left(107^{*}\right)$ \\
\hline & TLS & 2622 & 171 \\
\hline Orientation & $\mathrm{DFN}^{+}$ & $\begin{array}{l}\mathrm{m}=000^{\circ} / 05^{\circ} \\
\mathrm{sd}=180^{\circ} / 05^{\circ}\end{array}$ & $\begin{array}{l}\mathrm{m}=000^{\circ} / 70^{\circ} \\
\mathrm{sd}=180^{\circ} / 15^{\circ}\end{array}$ \\
\hline \multirow[t]{2}{*}{ Length $[\mathrm{m}]$} & scanline & $0.03 / 0.65 / 11.6$ & \\
\hline & TLS & $0.14 / 1.00 / 9.31$ & \\
\hline \multirow[t]{2}{*}{ Area $\left[\mathrm{m}^{2}\right]$} & TLS & $0.05 / 1.02 / 46.42$ & \\
\hline & TLS & $\operatorname{Ex}\left(\frac{1}{\lambda}=0.5\right)$ & \\
\hline Radius $[\mathrm{m}]$ & $\mathrm{DFN}$ & $\begin{array}{c}\operatorname{Ex}\left(\frac{\hat{1}}{\lambda}=0.5\right) \\
\operatorname{LN}(\bar{y}=0.4 \\
\left.y_{\sigma}=0.75\right) \\
\operatorname{PL}\left(\mathrm{x}_{\min }=0.65\right. \\
\quad \alpha=4)\end{array}$ & $\begin{array}{c}\operatorname{Ex}\left(\frac{1}{\lambda}=0.5\right) \\
\operatorname{LN}(\bar{y}=0.4 \\
\left.y_{\sigma}=0.75\right) \\
\operatorname{PL}\left(\mathrm{x}_{\min }=0.65\right. \\
\quad \alpha=4)\end{array}$ \\
\hline \multirow[t]{2}{*}{$\mathrm{P}_{10}\left[\mathrm{~m}^{-1}\right]$} & scanline & $1.5\left(3.4^{*}\right)$ & $0.2\left(1.1^{*}\right)$ \\
\hline & TLS & 1.2 & \\
\hline $\mathrm{P}_{21}\left[\mathrm{~m}^{-1}\right]$ & TLS & 1.9 & \\
\hline \multirow{2}{*}{$\mathrm{P}_{32}\left[\mathrm{~m}^{-1}\right]$} & TLS & 2.4 & \\
\hline & DFN & $0.65 ; 2.4 ; 6.5$ & $0.065 ; 0.24 ; 0.65$ \\
\hline \multirow{2}{*}{$\begin{array}{c}\text { Termination } \\
\text { probability } \\
{[0-1]}\end{array}$} & scanline & 0.75 & \\
\hline & DFN & 0.75 & \\
\hline Aperture $[\mathrm{m}]$ & DFN & $\begin{array}{c}\text { No }(\mathrm{m}=0.002 \\
\left.x_{\sigma}=0.001\right)\end{array}$ & $\begin{array}{c}\text { No }(\mathrm{m}=0.002 \\
\left.x_{\sigma}=0.001\right)\end{array}$ \\
\hline \multicolumn{4}{|c|}{$\begin{array}{l}{ }^{*} \text { After sampling orientation bias correction }(\text { Terzaghi } 1965) \\
+ \text { Bivariate normal distribution, m=mean pole trend/plunge, sd=standard deviation trend/plunge } \\
\text { probability distributions: Exponential }(\text { Ex }): \quad f(x)=\lambda \exp (-\lambda x) ; \quad \text { Log-normal }(L N): f(x) \\
\frac{1}{x \ln (10) y_{\sigma} \sqrt{2 \pi}} \exp \left[-\frac{1}{2}\left(\frac{\log x-\bar{y}}{y_{\sigma}}\right)^{2}\right] \text {; Power-law }(P L): \quad f(x)=\frac{\alpha-1}{x_{\min }}\left(\frac{x}{x_{\min }}\right)^{-\alpha} ; \text { Normal }(\text { No): } f(x) \\
\exp \left[-(x-m)^{2} / 2 x_{\sigma}^{2}\right] / \sqrt{2 \pi x_{\sigma}^{2}}\end{array}$} \\
\hline
\end{tabular}



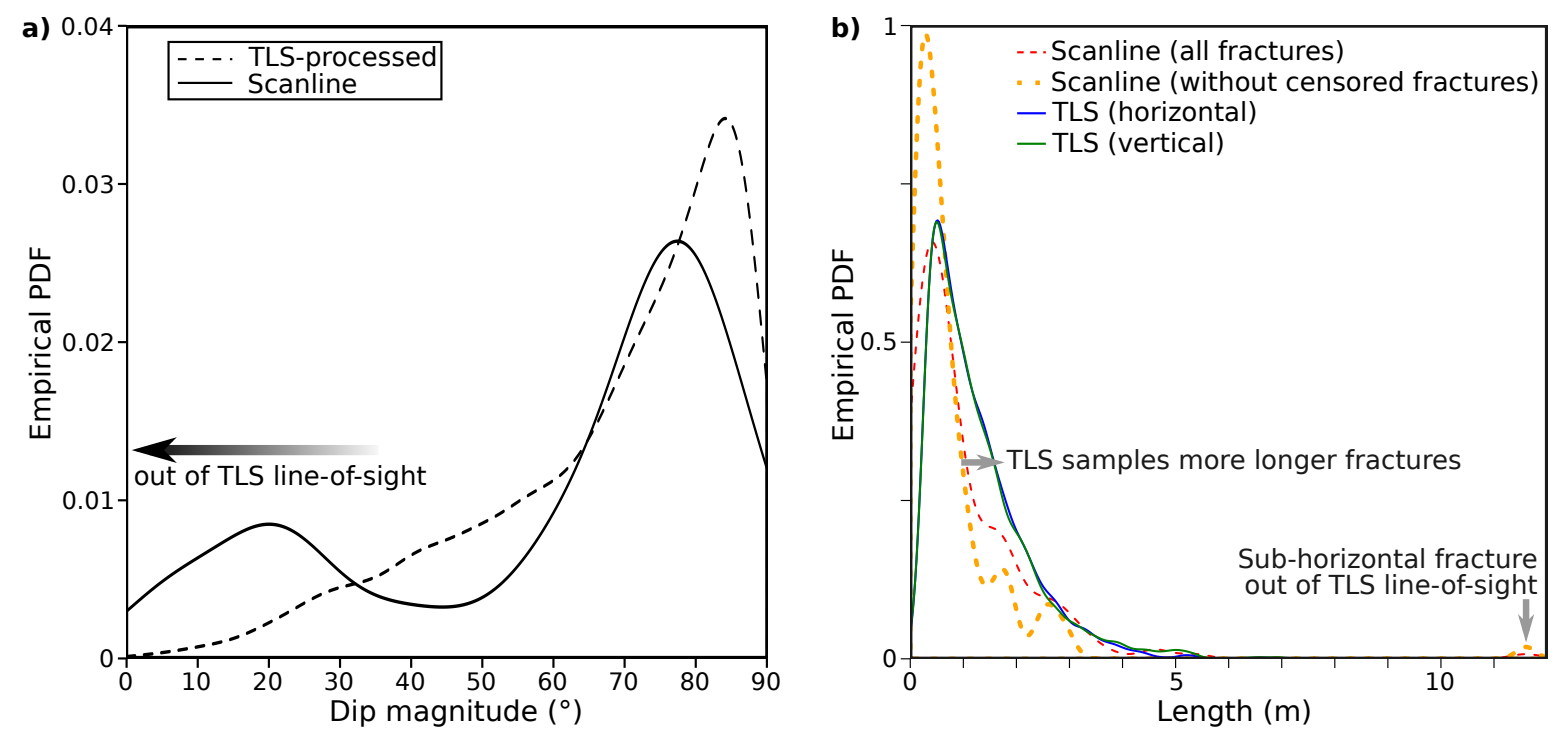

Figure 2.6: a) Empirical probability density function (PDF) of the dip magnitude for the scanline (corrected for Terzaghi sampling bias) and TLS-processed datasets. b) Empirical PDF of fracture length for the scanline (with and without censored fractures) and TLS-processed fractures (measured in horizontal and vertical directions).

mended by Priest (1993). The orientations of the steeply-dipping and gently-dipping column-forming fractures can thus be modelled using bivariate normal distributions (Table 2.1).

\subsubsection{Connectivity (Scanline) and Length (TLS and Scanline)}

The scanline dataset indicates that fractures are highly connected (fracture connectivity is not quantifiable from the TLS-processed dataset). Overall, $60 \%$ of the fractures where both terminations are observed terminate on another fracture at both ends, and an additional $30 \%$ on one end, yielding a termination probability of $75 \%$ (Table 2.1).

Scanline and TLS-processed datasets show a large range of fracture lengths about medians of 0.65 and $1 \mathrm{~m}$, respectively (Table 2.1. Figure 2.6b). The TLS-processed fractures are longer overall than those measured on the scanline due to sampling biases (see Section 2.3.3). The longest fractures measured on the scanline and TLS-processed dataset $(\sim \geq 5 \mathrm{~m})$ are steeply dipping $\left(>70^{\circ}\right)$. Moreover, numerous censored fractures (i.e. reported lengths are minimums) measured on the scanline are steeply dipping. By contrast, a long $(11.6 \mathrm{~m})$ sub-horizontal column-forming fracture was measured on the scanline, which was not detectable by the TLS processing.

Fracture radius follows either a negative exponential or a log-normal distribution. On sub-horizontal virtual trace maps parallel to the lower breccia, including a trace map at the manual scanline location, these two distributions have similar $\mathrm{K}-\mathrm{S}$ test results (with median D of 0.07 and 0.08 , and median significance of 0.99 and 0.92 , for the exponential and log-normal distributions, respectively), while power-law distributions 


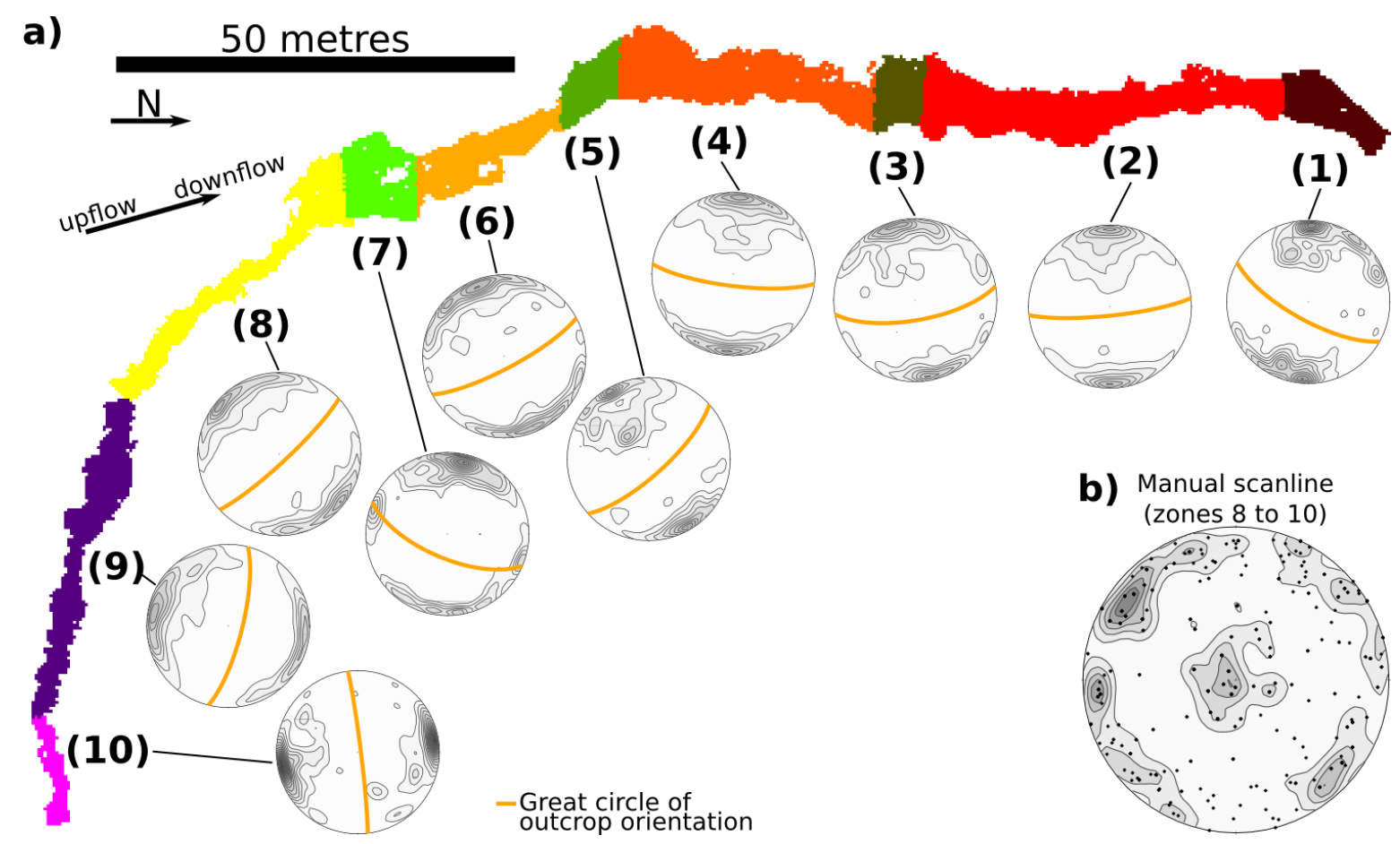

Figure 2.7: Orientation of column-forming fractures in relation to outcrop orientation. a) Plan view of the outcrop subdivided into 10 zones of constant orientation, accompanied by a stereonet of TLS-processed fracture orientations (Fisher density of pole to planes), and approximative lava flow direction. The outcrop orientation is plotted for each zones on the stereonets as a great circle. b) Stereonet with poles to planes sampled on the manual scanline (corrected for the Terzaghi orientation sampling bias). The scanline was measured in zones 8-10. All stereonets are Schmidt lower hemisphere projections, with Fisher density contouring spacing of $1 \%$. 


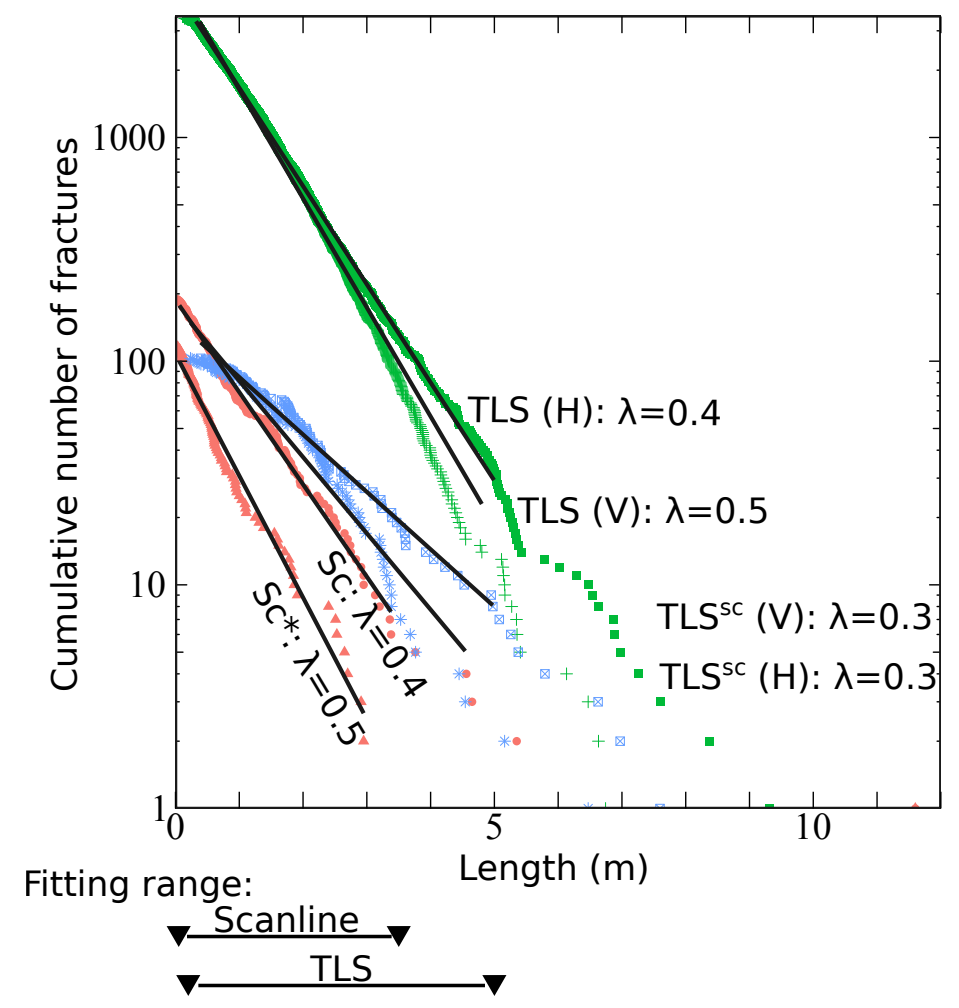

Figure 2.8: Complementary cumulative distribution function (CCDF) of fracture length in log-linear scale for fractures measured on the manual scanline (with $(S c)$ and without censored fractures $\left(S c^{*}\right)$, orange symbols) and processed from the TLS (over the entire outcrop (TLS; green symbols) and in the manual scanline area (TLS $S^{s c}$; blue symbols)) measured in sub-vertical $(V)$ and sub-horizontal $(H)$ orientations. Coefficients $\lambda$ of the linear trends within truncation limits provides the exponential distribution parameter. All adjusted correlation coefficients are $>0.98$, except the horizontal TLS-processed dataset in the scanline area $\left(\mathrm{TLS}^{s c}\right)$ which is 0.94 .

always present poorer fits (median D of 0.15 and median significance of 0.31 ). Subvertical trace maps orthogonal to the lower breccia are under-constrained because of the limited number of fractures intersected along 8-15 m-long trace maps, but also have better fits to negative exponential and log-normal distributions than to power-law distributions. Sturzenegger and Stead (2009b) showed that the lower bound of the lognormal length distributions of TLS-processed fractures differs at different observation scales, and recommended using an exponential distribution. Given the limited range of observations of fracture radius at Happy Valley (1.5 orders of magnitude), we also favour the negative exponential distribution over the log-normal distribution because it has only one parameter, rather than the log-normal distribution which has two. Using an exponential instead of a log-normal distribution in conditions where both fit the data similarly well avoids over-parametrisation.

The exponential parameter $\frac{1}{\lambda}$ of the fracture radius evaluated from sub-horizontal trace maps (hence from fracture radius chords, Figure $2.4 \mathrm{~b}$ ) ranges between $0.4-0.6 \mathrm{~m}$ with a median of $0.5 \mathrm{~m}$ (Table 2.B.1 parameters of the power-law and log-normal 
distribution fits are also included for comparison). There is no clear correlation between variations of probability distribution parameters and the location of the measurement within the lava-flow, either along flow or from base to top.

The preferred exponential distribution of fracture length (and thus radius) is confirmed by the scanline measurements. The complementary cumulative density functions (CCDFs) of fracture length measured on the scanline (with and without censored fractures), TLS-processed dataset, and on the subset of TLS-processed fractures in the manual scanline zone, all show a good fit to an exponential distribution (straight line in a log-linear plot; Figure 2.8). In addition, the exponential coefficients $\lambda$ are similar for all these subsets $\left(0.3-0.5 \mathrm{~m}^{-1}\right)$.

\subsubsection{Variability of Fracture Area Between Breccia and Blocky Facies}

Straight to curved columnar fractures either stop at the massive/breccia interface, or bifurcate in the mechanically weak and heterogeneous breccias (Figure 2.2). There are few exposures of the lower breccia at Happy Valley, but observations suggest that the fracture propagation is less affected by breccias with small clasts than with large clasts.

Fractures in the upper breccia zone, at the northern end of the outcrop, are smaller than in the underlying blocky zone, as revealed by the empirical probability density function (PDF) of fracture area (Figure 2.9c). While the median fracture area is similar in both the blocky and upper breccia zones ( 0.8 and $1.1 \mathrm{~m}^{2}$, respectively), only $4 \%$ of fractures in the breccia exceed $5 \mathrm{~m}^{2}$ in area, versus $15 \%$ in the blocky zone.

\subsubsection{Linear Fracture Intensity: $\mathrm{P}_{10}$}

$\mathrm{P}_{10}$ measured along a series of virtual scanlines intersecting the TLS-processed dataset varies between $0.7-1.7 \mathrm{~m}^{-1}$, with a mean of $1.2 \pm 0.1 \mathrm{~m}^{-1}$ (one standard deviation; Figure 2.10, Table 2.1). Sub-horizontal virtual scanlines show no clear relationship between the $\mathrm{P}_{10}$ value and location within the lava flow either from the base to the top of the blocky part of the outcrop, nor in the breccia intersected in zones 2 and 4 (Figure 2.7). There are no observed lateral variations of $\mathrm{P}_{10}$ between the up-flow and down-flow parts of the outcrop, as seen on sub-vertical virtual scanlines which sample the entire thickness of the outcrop. Steeply-dipping column-forming fractures measured along the manual scanline have a slightly higher $\mathrm{P}_{10}\left(1.5 \mathrm{~m}^{-1}\right)$ than the TLS-processed dataset, which reflects the additional sampling of short fractures. 
a)

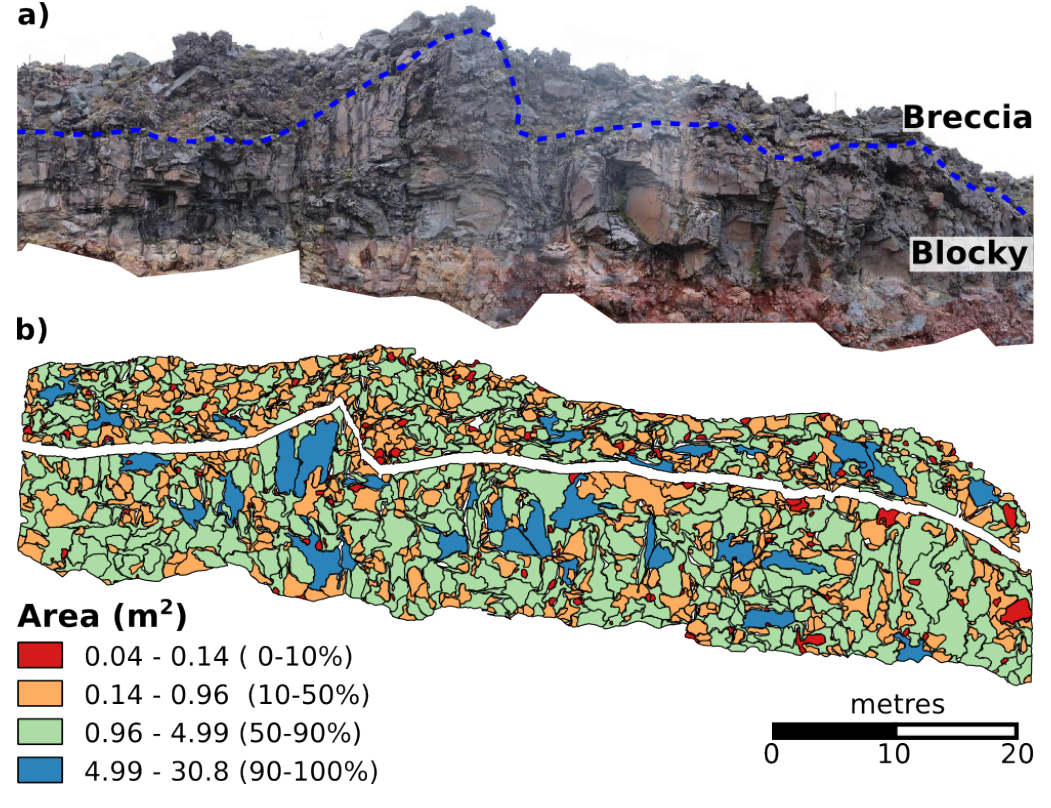

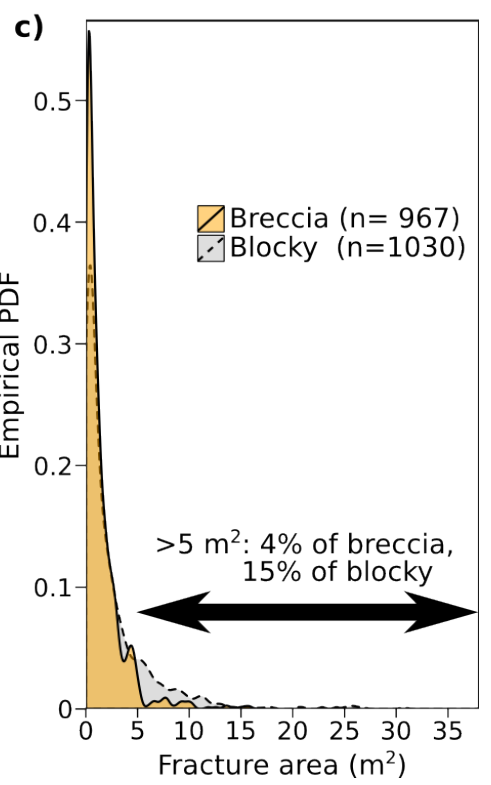

Figure 2.9: Variation of fracture area between the blocky and upper brecciated zones at the northern end of the outcrop. a) Panoramic photograph. b) TLS-processed fractures coloured by fracture area. The brecciated and blocky zones are separated by a white band to help visualisation. c) Empirical PDF of the fracture area in the breccia and blocky zones.

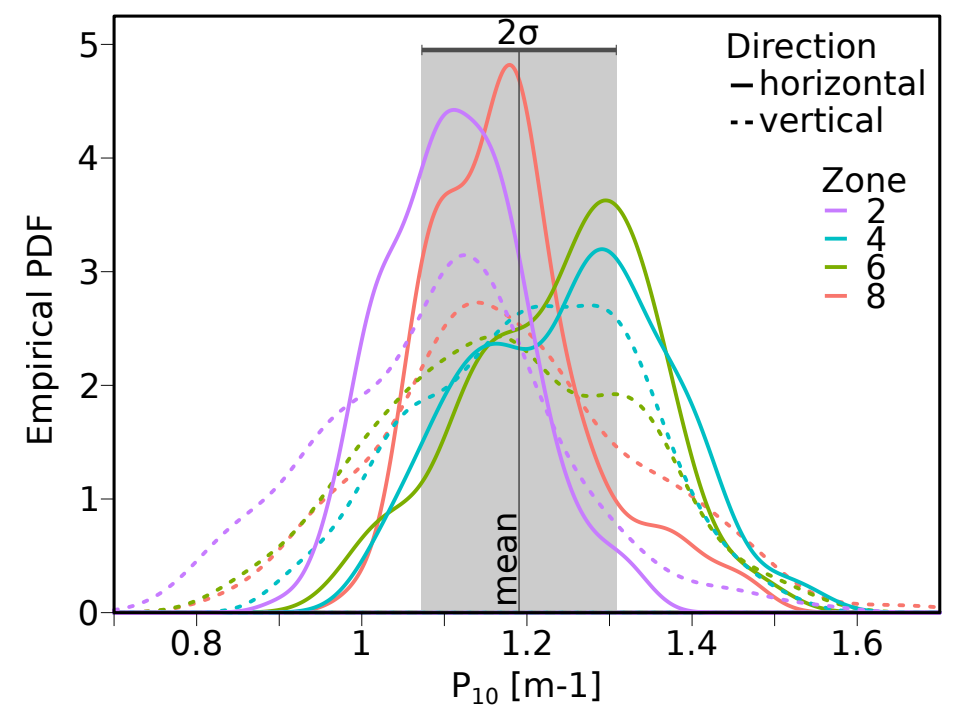

Figure 2.10: Empirical PDF of linear fracture density $\left(\mathrm{P}_{10}\right)$ along a series of virtual scanlines in zones $2 ; 4 ; 6$ and 8 (Figure 2.7), measured every $10 \mathrm{~cm}$ parallel (subhorizontal) and perpendicular (sub-vertical) to the lower breccia. Sub-horizontal scanlines have a mean fracture density of $1.2 \mathrm{~m}^{-1}$ and standard deviation of $0.1 \mathrm{~m}^{-1}(\sigma)$, shaded in grey. 


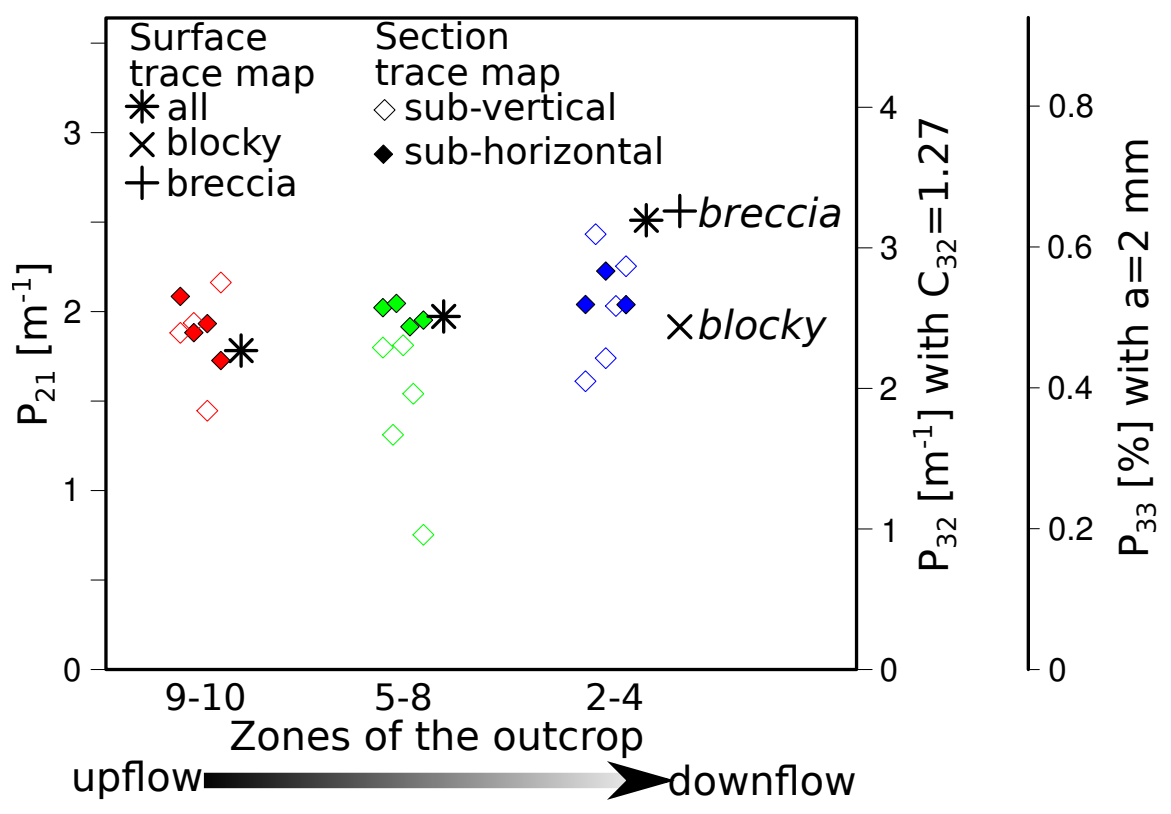

Figure 2.11: Measured $\mathrm{P}_{21}$ (areal intensity) and estimated $\mathrm{P}_{32}$ (volumetric intensity) and $\mathrm{P}_{33}$ (fracture porosity) parameters for each zone of the outcrop. Measurements are made over the entire outcrop surface (Surface trace map; Equation 2.2), and on trace maps perpendicular (sub-vertical) and parallel (sub-horizontal) to the lower breccia (Section trace map; Equation 2.3). $\mathrm{P}_{32}$ and $\mathrm{P}_{33}$ parameters assume $\mathrm{C}_{32}=1.27$ and aperture $=2 \mathrm{~mm}$ (Equations 2.4 and 2.5). Zone numbers refer to Figure 2.7a; zones 2-4 measured on surface trace maps are subdivided by facies (breccia or blocky).

\subsubsection{Areal $\left(\mathrm{P}_{21}\right)$ and Volumetric $\left(\mathrm{P}_{32}\right)$ Intensity, and Fracture Porosity $\left(\mathbf{P}_{33}\right)$}

The $\mathrm{P}_{21}$ of the steeply-dipping column-forming fractures for the blocky zone of this outcrop is estimated to be $\sim 1.8-2.0 \mathrm{~m}^{-1}$ (Table 2.1. Figure 2.11). The $\mathrm{P}_{21}$ value evaluated directly from the surface of the outcrop is $1.8-2.0 \mathrm{~m}^{-1}$ in the blocky parts of the outcrop. $\mathrm{P}_{21}$ in the upper breccia of zones $2-4$ is $2.6 \mathrm{~m}^{-1}$, reflecting smaller fracture sizes than in the blocky region, and hence the higher total fracture edge length. The $\mathrm{P}_{21}$ derived from virtual trace maps parallel to the lower breccia is similar $\left(1.7-2.2 \mathrm{~m}^{-1}\right)$ to those estimated from the surface of the outcrop, and does not exhibit any discernible variation related to the position in the outcrop (Figure 2.11). $\mathrm{P}_{21}$ values derived from sub-vertical trace maps orthogonal to the lower breccia are less constrained than those measured from trace maps parallel to the lower breccia, because of the lower number of intersected fractures, but have a similar range and median of $\mathrm{P}_{21}$ values. The two lowest $\mathrm{P}_{21}$ values, in zones 5 to 8 , are measured in the narrowest part of the outcrop (10 $\mathrm{m}$ high) and are thus the least reliable.

Calculation of the $\mathrm{P}_{33}$ parameter includes the $\mathrm{C}_{32}$ parameter and fracture aperture (Equation 2.5). Calculation of the $\mathrm{C}_{32}$ parameter requires the assumption that fracture sets define clusters with a mean orientation, or that orientation is isotropic 
(Wang, 2005). In the Happy Valley outcrop, fracture strikes lack a mean orientation. Although there are two sets of dip magnitude, we use the $\mathrm{C}_{32}$ value of 1.27 applicable to the isotropic case as an approximation (Wang, 2005). The corresponding $\mathrm{P}_{32}$ is $2.3-2.5 \mathrm{~m}^{-1}$ (Equation 2.4). Sparse scanline measurements show that the fracture aperture is $\sim 2 \mathrm{~mm}$ (aperture is not measurable from the TLS as it is smaller than the point cloud resolution). The resulting $\mathrm{P}_{33}$ value for steeply dipping column-forming fractures is $0.0046-0.005$, equivalent to $\sim 0.5 \%$ fracture porosity (Figure 2.11).

\subsection{Discussion}

\subsubsection{Applicability of TLS for Fracture Characterisation in Volcanic Rocks}

Processing of the TLS point cloud can provide a detailed description of the fracture distribution over a large outcrop, with a short acquisition time (a few hours) and in areas not easily accessible due to outcrop height or other hazards. The TLS technique is limited by the portability of the equipment, including the installation of several temporary GPS stations near the acquisition site. The choice of outcrop is thus key to a successful TLS campaign, from an operational and sampling point of view. Outcrops with variable exposure orientations ensure that multiple fracture sets are sampled, as recommended by Hudson and Priest (1983). Furthermore, an outcrop curving towards the TLS acquisition site, and thus at the same distance to the acquisition site in all directions, is particularly favourable as it maintains a similar accuracy and point density throughout the point cloud.

Shape detection algorithms were successful in extracting fractures from the TLS point cloud in this blocky lava flow, in which fractures can be approximated by a coplanar 3-D set of points. Indeed, the majority of column-forming fractures at Happy Valley are steeply dipping and form 3-D relief in the line-of-sight of the TLS device. In favourable cases, additional TLS acquisitions from a high elevation relative to the outcrop would help in the detection of gently-dipping fractures. However, the delineation of in-plane fractures, and more generally those that lack significant 3-D-relief, is very limited. The similarity of the fracture processing results obtained with two different methods (RansacSD and Facets; Appendix 2.A), with various degrees of user input, further supported by manual scanline measurements, increases our confidence in the resulting fracture dataset and helps to evaluate the sampling biases of each sampling method.

Manual scanline and panoramic pictures are crucial for calibrating and validating fracture processing from the TLS point cloud. Although time-consuming, the manual scanline also yields detailed information on the geometry of fractures not detected by 
the TLS, and provides insights into the processes controlling the organisation of the fracture system. The panoramic pictures were quick to acquire (30-40 min per photograph) and constrain structures not measured by the scanline due to time constraints or inaccessibility.

\subsubsection{Column-forming Fracture Orientation and Size}

Column-forming fractures at Happy Valley are either steeply- or gently-dipping, i.e. subperpendicular or sub-parallel to the lower breccia, without preferred strike orientation. These orientations are similar to outcrop observations in previous studies (DeGraff and Aydin, 1987; Schaefer and Kattenhorn, 2004), and numerical models (Lore et al., 2000, 2001; Kattenhorn and Schaefer, 2008) for basalt lava flows, and result from a cooling front causing thermal stresses propagating inward from the base and the top of the lava flow.

TLS-processed fracture radii (modelled as disks) follow an exponential or a lognormal distribution; the exponential distribution is preferred here as it has only one parameter, and because it provides a good approximation of fracture length in the manual scanline and TLS-processed dataset. The robustness of this analysis is limited by the scale range of observations which spans less than two orders of magnitude. Fracture length measurements from thin sections would extend the range of observations to scales $<1 \mathrm{~cm}$ and strengthen results from outcrop-scale observations, assuming that fractures at both scales result from the same processes.

The exponential fracture length distribution found in the Happy Valley andesite is consistent with the statistical analysis reported on moderately-weathered outcropping andesites (Sari et al., 2010), in the vicinity of mid-oceanic ridges in volcanic lava flows (Carbotte and Macdonald, 1994), and in other non-volcanic rocks (Cruden, 1977; Hudson and Priest, 1979; Kulatilake et al., 1993; Aler et al., 1996; Priest and Hudson, 1981). Exponential length distributions have been related to a growth process controlled by a uniform stress distribution (Dershowitz and Einstein, 1988), where the propagation of fractures is a Poissonian (random) process. In a lava flow, the thermal stress is controlled by the advance of the cooling from the outside to the inside of the lava flow; at a given time, the thermal stress is thus uniform in the direction parallel to the borders of the lava flow, and changing gradually from the border to the inside of the flow. This stress distribution is not uniform but is more gradual than in anisotropic stress distributions controlled by tectonic processes.

Exponential or log-normal fracture length distributions imply that the system has characteristic length scales, either $\lambda$ or $\left(\bar{y}, y_{\sigma}\right)$, respectively (Table 2.1). In lava flows, fracture propagation is limited by the breccias at the boundaries of the flow, and the characteristic scales may thus relate to the dimensions of the lava flow (thickness, width and length). The length of column-forming fractures perpendicular to the lava flow di- 
rection has an upper bound at the thickness of the lava flow $(5-21 \mathrm{~m})$, which is reflected in the $10 \mathrm{~m}$ maximum length measured in the TLS-processed dataset. The length of column-forming fractures is further restricted by gently-dipping platy fractures that arrest or deflect column-forming fractures. Column-forming fractures parallel to the lower breccia tend to be shorter, although one $12 \mathrm{~m}$-long sub-horizontal fracture parallel to flow-banding has been measured on the manual scanline. In sedimentary formations, characteristic scale values have been linked to lithological layering (Odling et al., 1999; Ackermann et al. 2001). Further measurements in lava flows may be required to evaluate the impact of the lava-flow thickness on the fracture length distribution, and assess whether processes controlling fracture length in volcanic rocks are comparable to those operating in layered sedimentary systems (Schöpfer et al., 2011). The chemical composition of the lava, and hence its viscosity and thermal conductivity, as well as the temperature and phase of the cooling fluid (air, water or ice), impact the cooling rate of the lava flow (Lore et al., 2001; Conway et al., 2015) and may thus also control the scaling of fracture length. The scale of the exponential distribution has also been reported to reflect a feedback process during fracture growth (Renshaw and Park, 1997). Field observations Schaefer and Kattenhorn, 2004) and numerical models (Lore et al., 2000, 2001) have shown that neighbouring fractures develop a hierarchy of leading and trailing fractures, which interact with each other, and may cause such feedback process.

\subsubsection{Column-forming Fracture Intensity}

While the orientations and lengths of the column-forming fractures are well constrained from the 1-D and 2-D measurements, the fracture intensity in 3-D is less constrained. $\mathrm{P}_{21}$ and $\mathrm{P}_{32}$ fracture intensity parameters are traditionally measured from trace maps of the intersections between fractures and an outcrop face, i.e. the fracture edges, while TLS processing used here provides the location and geometry of fracture faces. The evaluation of $\mathrm{P}_{21}$ fracture intensity on the outcrop surface, although assumes that fractures are fully tessellated and share edges, is preferred to the virtual section trace maps because it uses the TLS-processed fracture dimensions directly. As the two $\mathrm{P}_{21}$ measurement techniques provide similar result (Figure 2.11), we are confident that the resulting values are representative of the outcrop.

The $2.4-2.5 \mathrm{~m}^{-1}$ fracture intensity $\mathrm{P}_{32}$ values relate only to the steeply-dipping column-forming fractures, as gently-dipping fractures were not sampled by TLS processing. Gently-dipping fractures are fewer and shorter (rarely $>3 \mathrm{~m}$ long, as indicated by the manual scanline) than steeply-dipping ones, so the $\mathrm{P}_{32}$ for gently-dipping fractures is a small fraction of the $\mathrm{P}_{32}$ for steeply-dipping fractures. Therefore, the estimated $\sim 0.5 \%$ fracture porosity for the steeply-dipping column-forming fractures is supplemented by an additional small contribution from the gently-dipping columnforming and platy fractures. 


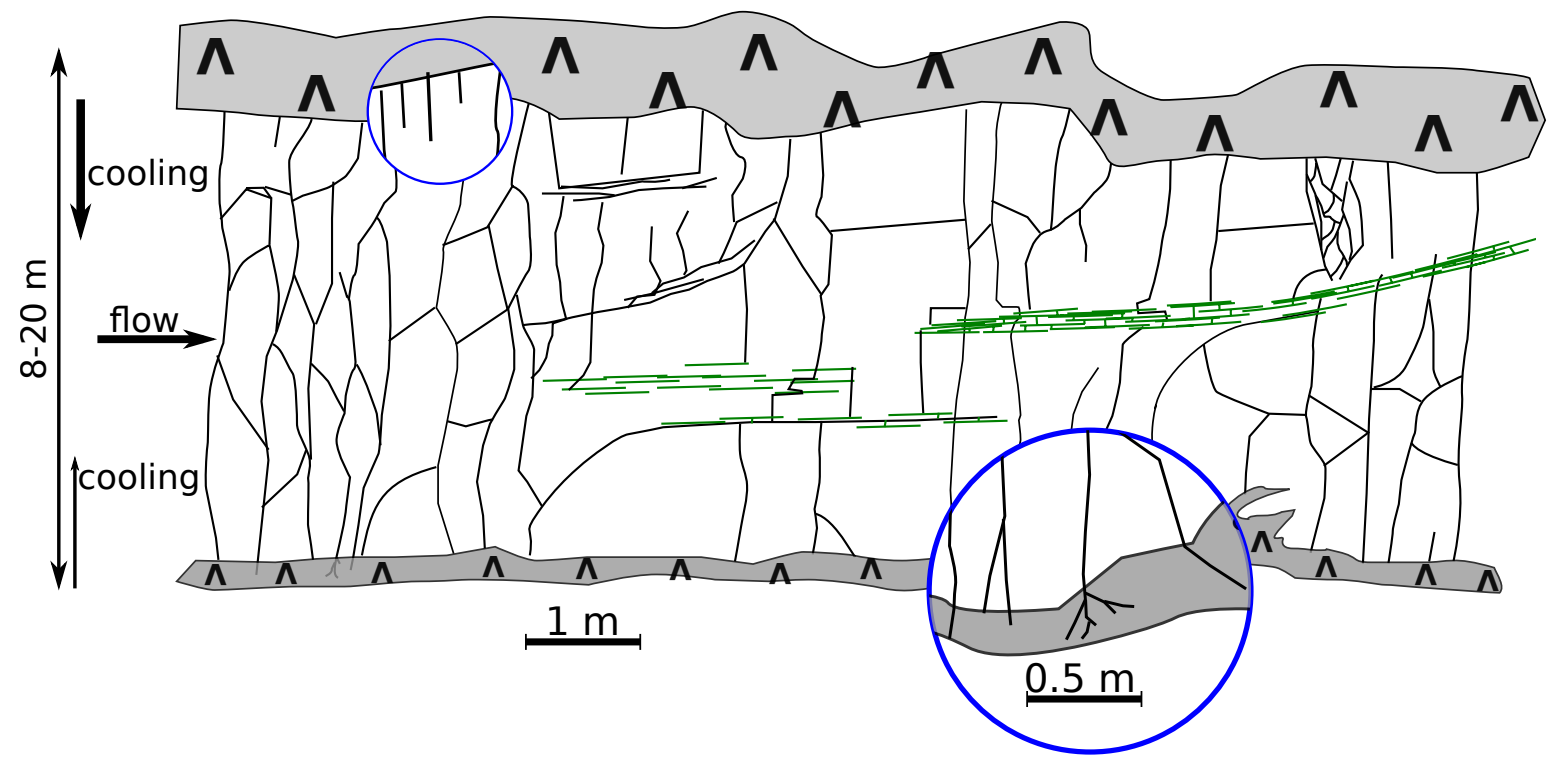

Figure 2.12: Interpretive diagram of the Happy Valley lava flow outcrop. Columnforming fractures are well connected within the blocky zone, with higher $\mathrm{P}_{32}$ for fractures perpendicular to the lower breccia than for those parallel to the flow direction. Metre-scale zones of high fracture density are associated with platy fractures (green lines). The high-porosity and friable breccias (shaded in grey) are partially connected to the blocky flow interior by those column-forming fractures that propagate into the breccia. The circled area are zoomed-in views of the breccia-blocky interface showing the higher density of column-forming fractures (commonly observed in lava flows, but inferred at Happy Valley due to the lack of exposure) and the fracture bifurcations in the breccias.

\subsubsection{Controls on the Geometry of Column-forming Fractures}

An interpretive diagram of the fracture system geometry within the Happy Valley outcrop highlights the high connectivity of column-forming fractures, and the role of platy fractures and breccias on the fracture system (Figure 2.12). Column-forming fractures commonly terminate at, or curve parallel to, the platy fractures, forming blocks that are well connected to each other. Platy fractures thus have a strong impact on the organisation of the fracture system. Even where platy fractures are not fully open and detectable, they may control deflection or arrest of column-forming fractures. Similarly, layering such as flow-banding, and dilatant fractures parallel to the lava flow, may interact with, and result in the termination of, column-forming fractures perpendicular to the lower breccia (Schaefer and Kattenhorn, 2004).

Where observed, the column-forming fractures either stop, or bifurcate at the blocky-breccia interface. This configuration provides connections for flow pathways between the blocky and the breccia sections of the lava flow. The breccia clasts are fitted by fracture planes smaller than the column-forming fractures in the blocky zone. $\mathrm{P}_{10}$ fracture density values do not vary between the blocky and breccia facies (Figure 
$2.10 \mathrm{a})$, however, the breccia zone in the northern part of the outcrop has fewer large fractures $(>5 \mathrm{~m}$ ) than the blocky zone (Figure 2.9p. This difference in fracture size is reflected in the $\mathrm{P}_{21}$ value which is higher in the breccia than in the blocky zone. $\mathrm{P}_{10}$ fracture intensity does not include the fracture size, and the $\mathrm{P}_{21}$ measure is preferred here as the intensity measure.

Fracture density and size vary locally within the Happy Valley outcrop, although these variations are not systematic up or along the exposure (Figure 2.10). Outcrop observations (DeGraff et al., 1989), supported by numerical models of fracture density in basalt lava flows (Lore et al., 2001), indicate that fracture density is typically higher near the margins than in the interior of the flow, due to the faster cooling rate closer to the margins. Similarly, observations from the Meads Wall dyke (400 m from Happy Valley) and similar lava flows located on Ruapehu show that this higher density occurs within $0.1-0.5 \mathrm{~m}$ of the cooling surfaces. This higher fracture density near the margins of breccias is not observed at Happy Valley, which may be due to the limited data available from the lava flow margins.

\subsubsection{Upscaling Fracture Geometries to Volcano scale}

Upscaling fracture geometries from a single lava flow to an entire volcano such as Ruapehu, or to reservoirs hosted in buried composite volcanic edifices such as inferred for the Rotokawa Geothermal Field, is not trivial. The geological structure of composite volcanoes such as Ruapehu and Tongariro is inherently complex due to the polygenetic nature of the magma systems that feed them over tens to hundreds of kyrs Davidson and De Silva, 2000), with different magma that may be tapped for each eruption (e.g., Gamble et al., 2003). Therefore, the flanks of composite volcanoes may comprise sequences of lava flows that each have different compositions and petrogenetic properties from adjacent flows. The style of eruption similarly covers a wide range, from effusion of lava flows to explosive fragmentation and emplacement of volcaniclastic material. The juxtaposition of rocks with different ages, compositions and eruption styles, and hence of fracture system geometries, is further complicated at volcanoes where eruptions have been sourced from spatially distinct vent locations, thus resulting in complex stratigraphic relationships (e.g. Tongariro; Hobden et al., 1996).

While the compositional range of Ruapehu lavas extends from $\sim 54-65$ wt. $\% \mathrm{SiO}_{2}$, andesite lavas with $\sim 58-60$ wt. $\% \mathrm{SiO}_{2}$ such as Iwikau at Happy Valley are the most common eruptive product (Price et al., 2012; Conway et al., 2016). Hackett and Houghton (1989) concluded that sheet lavas and associated autobreccias comprise $\sim 88$ vol. \% of the edifice, although Conway et al. (2016) argues that this figure is likely overestimated based on a new historical reconstruction of Ruapehu. Other volumetrically minor deposits that form the Ruapehu edifice include hyaloclastite breccias, pyroclastic rocks, alluvium, and debris avalanche deposits which likely have distinct 
fracture properties compared to the Happy Valley lava flow.

Due to the interaction between lava and ice, 48-16 ka-old flows of the Mangawhero Formation are thicker $(10-100 \mathrm{~m})$ than those of the Whapakapa Formation (typically $<10 \mathrm{~m}$ ), and characterised by fine-scale cooling fractures on their lateral margins and ubiquitous platy fractures in their interiors (Conway et al. 2015, 2016). Overall, lavas of the Mangawhero and Whakapapa Formations which have been in contact with ice have similar column-forming fracture average length $(1 \mathrm{~m})$, and slightly higher $\mathrm{P}_{10}$ values $\left(3-10 \mathrm{~m}^{-1}\right)$ than those measured at Happy Valley due to higher cooling rates (Conway et al., 2015). The small spacing and short length of platy fractures at Happy Valley is similar to that reported by Conway et al. (2015), but these fractures are less pervasive at Happy Valley than in lavas which cooled in contact with ice.

The Happy Valley flow is part of a Holocene low-volume coherent andesite lava flow that was emplaced on a relatively shallow slope gradient on Ruapehu. As such, it is a representative site for constraining the fracture geometries and properties of Whakapapa Formation lavas, and similar sheet flows and autobreccias that make up the older formations at Ruapehu at mid-low elevations ( $<1600 \mathrm{~m}$, Happy Valley's altitude) where the ice extent was limited.

\subsubsection{Potential Effects of the Fracture System on Reservoir Permeability}

The column-forming fractures observed on lava flow outcrops are at least partially retained during burial and further exhumation, as described in epithermal deposits hosted in andesite and rhyolite lavas in the Coromandel Peninsula, New Zealand (Brathwaite et al. 2001; Zuquim and Rowland, 2013). In these systems, some veins and hydrothermal breccias (that represent paleo-fluid flow pathways) formed along column-forming fractures and flow-banding, indicating that these structures can control permeability.

Breccias at Happy Valley are weak, heterogeneous, and of high matrix porosity. Therefore, breccias affect fracture propagation by forming a mechanical interface, and also have a high permeability potential. The resulting flow pathways in breccias may be better developed laterally, along the borders of the lava flow, than vertically, as these breccias have limited thickness. Such lateral flow pathways controlled by brecciated margins of buried rhyolitic lava flows are suggested at the Tauhara and Ohaaki geothermal fields, TVZ (Milloy and Lim, 2012, Mroczek et al., 2016). Connections between breccias of adjacent lava flows have the potential to further enhance lateral connectivity and permeability at the scale of several lava flows.

Conversely, permeability measurements in boreholes in the basaltic volcanic island of Mayotte (Comoros) indicated that most of the fluid flow occurs within a fractured lava flow interior rather than in the brecciated zone (Lachassagne et al., 2014). This suggests that in some cases, the low strength and cohesion of breccias make them 
more vulnerable than the blocky interior to erosion (while on the surface), compaction, hydrothermal alteration, and other processes that could decrease permeability (Custodio, 2007). Hydrothermal alteration within the andesite-hosted Rotokawa Geothermal Field is generally more intense in the pseudo-breccias and breccias than the massive lavas, presumably due to greater fluid-rock interactions Andersen, 2011) associated with rapid precipitation kinetics and high flow rates. Intense breccia alteration in reservoirs can decrease permeability in different ways: directly via mineral deposition, which decreases porosity and associated permeability (a relationship quantified for the Rotokawa geothermal field by Siratovich et al. 2014 ), or associated with the deformation of highly altered rocks which can result in a decrease of porosity (and hence permeability) even with a decreased strength (Siratovich et al., 2016). Even for weakly or moderately altered breccias, the higher tortuosity of flow pathways caused by the clasts may decrease permeability (Heap et al. 2014) compared to a network of planar column-forming fractures. The entire history of reservoir andesites, from emplacement at the surface to reservoir rock, thus influences permeability which can vary at short length scales (see discussion in Siratovich et al., 2016; Hernandez et al., 2015).

Similar to faults Rowland and Simmons, 2012, breccias may thus also form conduits or barriers to fluid flow. Where breccias decrease permeability, they may compartmentalise reservoirs. Such compartmentalisation is observed in the Rotokawa reservoir (Hernandez et al., 2015), although it is not clear whether the compartmentalisation is related to faults (McNamara et al., 2015; Sherburn et al., 2015); the internal structure of the $1 \mathrm{~km}$-thick series of lavas, pseudo-breccias and breccias; or a combination of both.

\subsubsection{Fracture System Modelling for Happy Valley-type Lava Flows}

Figure 2.13 presents DFN models of the blocky zone at Happy Valley based on three $\mathrm{P}_{33}$ values and the three length distributions considered in this paper (exponential, log-normal and power-law, even if the power-law distribution does not fit the data well; all parameters are listed in Table 2.1). The DFNs are composed of two fracture sets: sub-vertical and sub-horizontal (Figure 2.13a). To illustrate differences between models, a virtual trace map dipping at $45^{\circ}$ sampling both fracture sets displays fracture traces intersecting the plane in each model (Figure 2.13b). Two parameters are used to compare the fracture networks: the volume of fractures intersected by the plane, and the fracture volume connected to the trace map plane as a percentage of the entire fracture network. In a reservoir in which boreholes intersect fractures, the intersected fracture volume would correspond to the volume of fluids directly accessible, while the connected volume would correspond to the volume of fluids potentially recoverable.

Increasing $\mathrm{P}_{33}$ raises the volume of fractures intersected by the trace map, as well 

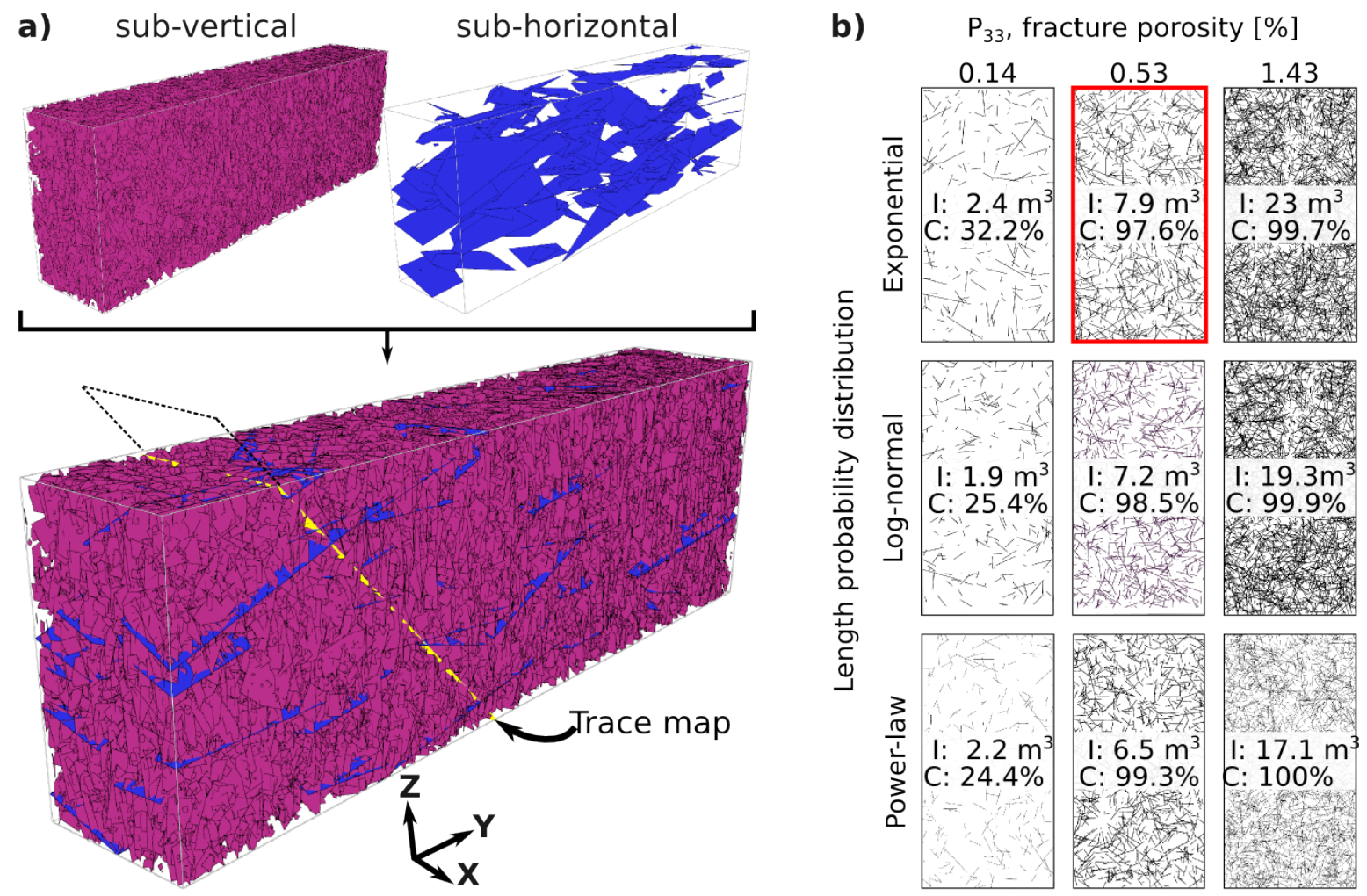

Figure 2.13: Examples of DFN models. a) DFN models are composed of two fracture sets (sub-vertical and sub-horizontal), with a 10:1 ratio of areal intensity $\mathrm{P}_{32}$, in a volume of $36366 \mathrm{~m}^{3}$ (dimensions $15 \times 86 \times 28 \mathrm{~m}$ ). The trace map is a plane dipping $45^{\circ}$ which samples the fractures intersecting the plane. b) Trace maps showing the intersection of the fractures with the trace map plane with various fracture length distributions and fracture porosity $\left(\mathrm{P}_{33}\right)$. I indicates the total volume of fracture intersecting the trace map, and $C$ the fracture volume connected to the trace map as a percentage of the entire fracture network. The model generated with the parameters best characterising the Happy Valley outcrop (exponential length distribution and $0.53 \% \mathrm{P}_{33}$ ) is outlined in red. 
as the level of connectivity: nearly all (>97 vol. \%) fractures are connected to the trace map at $0.53 \%$ porosity and above, which is the $\mathrm{P}_{33}$ value estimated at Happy Valley (Figure 2.13b). The volume of fractures intersected by the trace map plane is smallest with a power-law length distribution for $\mathrm{P}_{33} \geq 0.53 \%$, and highest with an exponential length distribution. The length distribution also affects the level of connectivity, particularly for $\mathrm{P}_{33}$ of $0.14 \%$ where the network is only partially connected.

A total $\mathrm{P}_{33}$ value of $0.53 \%$, together with the other geometrical parameters evaluated in this paper (Table 2.1), allows the fracture system to be almost completely connected (97.6\%; Figure 2.13 b). While there is some uncertainty on the analysis of the three components of the $\mathrm{P}_{33}$ (namely the $\mathrm{P}_{21}, \mathrm{C}_{32}$ and aperture), the fact that the numerical model provides a high level of connectivity similar to outcrop observations increases confidence in the reliability of the estimated parameters. With this level of connectivity, it is interesting to note that the choice of fracture length distribution does not significantly affect the percentage of connectivity; however, it does affect the volume of fractures connected to the trace map. This 'total' $\sim 0.5 \%$ fracture porosity may be considered low compared to the matrix porosity (2-5\%; Appendix A), but the macro-fracture system of column-forming fractures is better connected and less tortuous than the micro-cracks and vesicles network governing matrix porosity, and hence likely exerts greater controls on the bulk permeability.

The most uncertain parameter of these DFN models, though critical to model permeability, is the fracture transmissivity derived from fracture aperture. We have used here a normal aperture distribution with a mean of $2 \mathrm{~mm}$ based on scanline measurements. Measurements of length/aperture ratios on site-specific reservoir analogues may provide better estimations; Gudmundsson et al. (2001) reports a $\sim 400$ ratio value measured on veins in basaltic rocks, whereas power-law relationships between fracture hydraulic aperture and length have also been used (Baghbanan and Jing, 2007). However, the fracture aperture actively contributing to the permeability depends on the roughness of the fracture planes (Ishibashi et al. , 2014). Fracture roughness itself depends on the connected matrix porosity and the resulting tortuosity, as shown by Heap and Kennedy (2016) in experimental measurements made for lava flows of the same formation as the Happy Valley outcrop. In addition, the $i n$-situ stresses in a reservoir would control the fracture aperture, e.g. by closing those fractures with little asperities orthogonal to $\sigma_{1}$ (maximum principal stress; Barton et al., 2013) or keeping open those with a high slip tendency (Townend and Zoback, 2000). For reservoir modelling applications, geometrical fracture parameters evaluated in this work should be adapted to fit available reservoir data. 


\subsection{Conclusion}

The Happy Valley lava flow outcrop studied here is representative of a young, 5-20 mthick lava flow of andesitic composition which flowed over a gently-dipping paleotopography. The acquisition, processing and analysis of the TLS point cloud outlined in this paper are suitable methods with which to study the fracture system of blocky lava flows formed during their emplacement and cooling. The automatic Facets algorithm extracting fracture planes permits a consistent processing of the dataset across a large outcrop, while requiring sufficient user input and calibration from other measurements to ensure its validity. The fracture system is composed of column-forming fractures which form the blocky part of the outcrop, flow-parallel clustered platy fractures which locally increase the fracture density and affect the propagation of column-forming fractures, and breccias at the margins of the lava flow. Column-forming fractures are oriented either sub-parallel or sub-perpendicular to the margins of the lava flow (i.e., sub-horizontal or sub-vertical in this case), without mean strike orientation. The length of steeply-dipping column-forming fractures, which is well sampled by the TLS, is best approximated by a scale-dependent exponential distribution. The areal and volumetric fracture intensities reveal a well-connected system within the blocky region, partially connected to the breccias, with a $\sim 0.5 \%$ fracture porosity. This seemingly low fracture porosity has a significant impact on the permeability within the lava flow due to the high connectivity and planarity of column-forming fractures. High porosity breccias may have a varied influence on permeability in reservoirs, depending on the burial and hydrothermal alteration history; breccia have the potential to promote lateral flow pathways, or to form flow barriers. Discrete fracture network models generated from fracture geometries estimated from the TLS analysis and manual scanline measurements yield a highly connected network, in agreement with outcrop observations.

\section{Acknowledgments}

We thank the Department of Conservation, Ngati Rangi and Ngati Hikairo iwi, and Ruapehu Ski Lifts for access to the outcrop. We are very grateful to P. Villamor for facilitating this study, and to D. Girardeau-Montaut for fruitful discussion and continuous improvement of the CloudCompare open-source software. Thanks are extended to Golder Associates for availability of the Fracman software.

\section{Supplementary data}

The high-resolution panoramic photographs are accessible on GNS Science's virtual media library (https://vml.gns.cri.nz; photographs number 181972-181974). En- 
largeable versions of the four panoramic photographs used in this study are also available at: www.gigapan.com/gigapans/191973; WWW.gigapan.com/gigapans/e1911e3d60bda65464de404762ff36b0; wWw.gigapan.com/gigapans/b7deaec44afe77fab919b671617b19bc and WWW.gigapan.com/gigapans/6fe29b5bc96c857ae365fb646ace5e84. 


\section{Appendix}

\section{A TLS Processing Workflow}

Figure 2.3 presents the processing workflow of the TLS used to extract fracture parameters. The first step consists of merging and aligning ("registering") individual scans using the GPS measurements (Buckley et al. 2008) in the RiScanPro software. At Happy Valley, the merged point cloud contains $>6.3$ million points. Merging of scans was adjusted with a plane patch filter algorithm resulting in a precision of $2 \mathrm{~cm}$. Few blind areas remain in the final dataset. Zones of the point cloud that captured vegetation or buildings were manually deleted in the CloudCompare v.2.7 software CloudCompare, 2016). Finally, the merged scan was sub-sampled to $\sim 1.4$ million points with a $4 \mathrm{~cm}$ regular spacing from which fracture planes were extracted (Figure 2.5b). This point cloud was vectorised, with the calculation on each point of the normal to the best-fitting plane in a local neighbourhood (radius of $0.15 \mathrm{~m}$; Figures $2.5 \mathrm{~b}$ and 2.A.1 b).

The extraction of fractures from the TLS point cloud is performed using the Facets plugin in CloudCompare with the fast marching method (Dewez et al., 2016, http: // Www.cloudcompare.org/doc/wiki/index.php?title=Facets_(plugin)). This method divides the point cloud into small patches of similar sizes, and then fuses those patches to generate "facets" using a fast marching front propagation. The Facets algorithm generates a series of point sets to which polygons are automatically fitted (Figure 2.A.1b). The orientation (dip and dip direction) and size (area, horizontal and vertical length of the bounding rectangle) is calculated for each polygon (Figures $2.4 \mathrm{~b}$ and 2.5c-e).

The choice of parameters and validation of the fracture processing with the Facets plugin is made in three ways. Firstly, the spatial extent and orientation of fractures processed from the Facets plugin are compared to the manual scanline where available; secondly, the spatial extent of the processed fractures are compared to the Gigapan panoramic photographs over the whole outcrop; and thirdly, the delineation of fractures is compared to the results of the RansacSD shape detection algorithm in CloudCompare (Schnabel et al. 2007) on a subset of the outcrop. The RansacSD method involves four steps (Figure 2.3): (1) vectorisation with SEFL (García-Sellés et al., 2011), which calculates the normals to each point and computes local geometrical parameters; (2) filtering of points of low planarity and high collinearity, which correspond to fracture edges in CloudCompare; (3) application of the RansacSD algorithm, looking for planes and spheres, which segments the point cloud into a series of subsets of points; and 


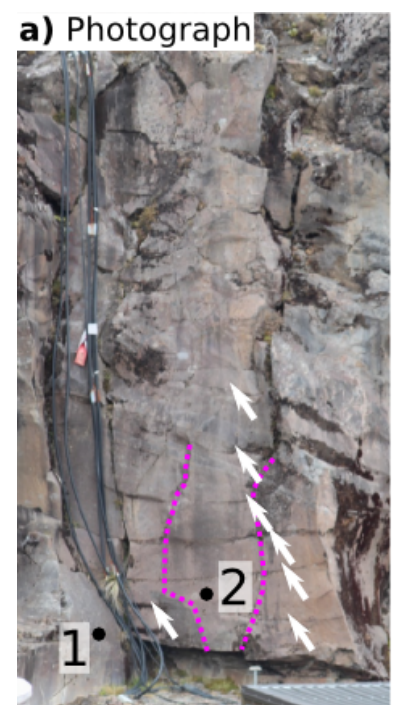

$\underline{1 \mathrm{~m}}$

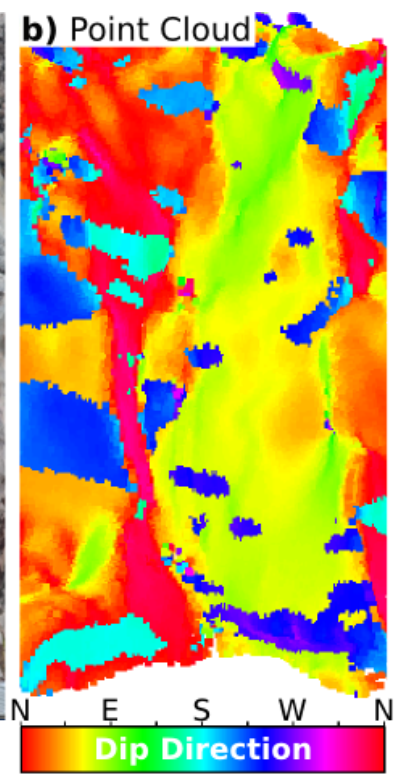

c) RansaçSD

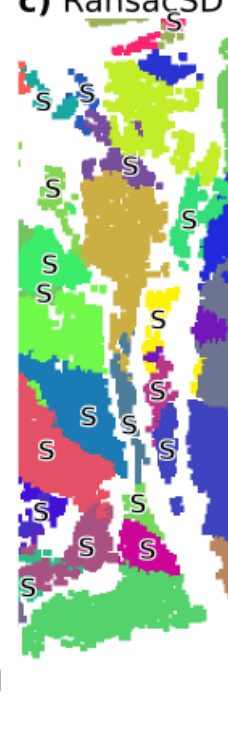

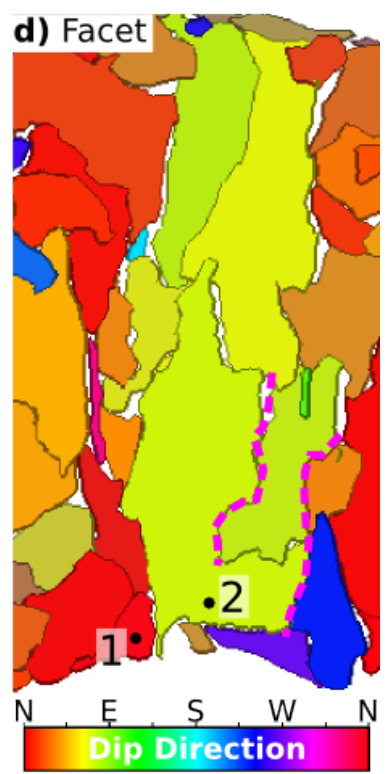

growth increment not detected by Facet

..... in-plane fracture partially detected by Facet

Figure 2.A.1: Appendix 2.A. Fracture processing of the TLS point cloud. a) Section of a Gigapan photograph acquired $100 \mathrm{~m}$ from the outcrop showing a column-forming fracture, with growth increments (white arrows) and in-plane fractures (purple dotted lines). b) TLS point cloud coloured by the local orientation. c) Point subsets separated with RansacSD. Fractures best fitted by a sphere are specified with an (S), others are recognised as planes. c) Planes delineated with Facets; parts of the in-plane fractures are recognised. Fracture " 1 " is oriented $80^{\circ} / 353^{\circ}$ and $80^{\circ} / 359^{\circ}$ on the scanline and TLS-processed datasets, respectively; Fracture " 2 " is oriented $78^{\circ} / 070^{\circ}$ and $82^{\circ} / 069^{\circ}$ on the scanline and TLS-processed datasets, respectively.

(4) post-processing, to manually check each subset, and subdivide them as necessary to match scanline and Gigapan photographs observations. As described by Ferrero et al. (2009), the filtering of fracture edges (steps 1 and 2) was necessary to obtain good results in RansacSD: without filtering, RansacSD generates very large fractures which requires significantly more post-processing, including removal of subsets generated along the fracture edges. The parameters used for the RansacSD step were iteratively selected by comparing the results to the scanline data and the Gigapan panoramas.

The RansacSD method has one major advantage compared to Facets: it detects spheres as well as planes, which allows the detection of curved fractures common in the Happy Valley outcrop. However, the additional steps of filtering fracture edges before running RansacSD, checking and modifying each subset individually, and fitting a plane to each subset to obtain the orientation and size of the fracture, is time-consuming. Conversely, the Facets method does not allow the delineation of curved fractures, but is significantly faster and provides results consistent with results from RansacSD, and 
from scanline and Gigapan photographs observations.

The regular resampling of the point cloud prior to the fracture extraction ensures that processing parameters are constant throughout the point cloud. Based on visual checks of processed fractures, a minimum of 25 points per fracture was imposed during the Facets processing to limit the false detection of noise as fracture planes. This threshold, equivalent to a fracture area of $0.04 \mathrm{~m}^{2}$, is similar to that recommended by Sturzenegger and Stead (2009a), and means that fractures with lengths of $\sim<0.2 \mathrm{~m}$ are not sampled (for a square fracture).

Figure 2.A.1b shows the orientation of the neighbourhood of each point (i.e. after vectorisation) and highlights the variations of dip and dip direction over a single fracture plane. These undulations are fitted by a series of spheres and planes by the RansacSD algorithm (Figure 2.A.1k), and a series of planes by the Facets algorithm (Figure 2.A.1d). Fracture orientations (dip magnitude and direction) of two fractures measured on the manual scanline and using the Facets algorithm only differ by a few degrees in both dip magnitude and direction, a discrepancy consistent with accuracies on TLS reported by Sturzenegger and Stead (2009a) and with errors on compass measurements.

\section{B Fracture Radius Parameters}

Fracture radius parameters estimated from trace map analysis of the TLS-processed dataset are presented in Table 2.B.1.

Table 2.B.1: Parameters (mean and 1 standard deviation) of the probability distribution fitted to the fracture radius from sub-horizontal and sub-vertical trace maps (i.e. parallel and perpendicular to the lower breccia, respectively), and near the manual scanline. n: number of scanline per zone, as defined in Figure 2.7.

\begin{tabular}{c|c|c|c|c|c|c|c}
\hline Direction & Zone & $\mathbf{n}$ & \multicolumn{5}{|c}{ Distribution } \\
\cline { 4 - 8 } & & & Exponential & Log-normal & \multicolumn{2}{c}{ Power-law } \\
\cline { 3 - 8 } & $2-4$ & 3 & $0.5 \pm 0.1$ & $0.8 \pm 0.1$ & $0.4 \pm 0.1$ & $0.7 \pm 0.1$ & $4.0 \pm 0.1$ \\
\hline \multirow{3}{*}{$\begin{array}{c}\text { sub- } \\
\text { horizontal }\end{array}$} & $5-8$ & 4 & $0.5 \pm 0.1$ & $0.7 \pm 0.1$ & $0.4 \pm 0.1$ & $0.6 \pm 0.1$ & $3.9 \pm 0.1$ \\
& $9-10$ & 3 & $0.5 \pm 0.1$ & $0.7 \pm 0.1$ & $0.5 \pm 0.1$ & $0.6 \pm 0.1$ & $3.7 \pm 0.2$ \\
\cline { 3 - 8 } & $2-10$ & 10 & $\mathbf{0 . 5} \pm \mathbf{0 . 1}$ & $\mathbf{0 . 7} \pm \mathbf{0 . 1}$ & $\mathbf{0 . 4} \pm \mathbf{0 . 1}$ & $\mathbf{0 . 6} \pm \mathbf{0 . 1}$ & $\mathbf{3 . 9} \pm \mathbf{0 . 2}$ \\
\hline \multirow{3}{*}{ sub-vertical } & $2-4$ & 5 & $0.5 \pm 0.1$ & $0.8 \pm 0.2$ & $0.4 \pm 0.1$ & $0.7 \pm 0.2$ & $3.9 \pm 0.1$ \\
& $5-8$ & 5 & $0.6 \pm 0.05$ & $0.8 \pm 0.1$ & $0.5 \pm 0.1$ & $0.7 \pm 0.1$ & $3.7 \pm 0.2$ \\
& $9-10$ & 5 & $0.6 \pm 0.1$ & $0.8 \pm 0.1$ & $0.5 \pm 0.1$ & $0.7 \pm 0.1$ & $3.7 \pm 0.2$ \\
\cline { 2 - 8 } & $2-10$ & 15 & $\mathbf{0 . 6} \pm \mathbf{0 . 1}$ & $\mathbf{0 . 8} \pm \mathbf{0 . 2}$ & $\mathbf{0 . 5} \pm \mathbf{0 . 1}$ & $\mathbf{0 . 7} \pm \mathbf{0 . 1}$ & $\mathbf{3 . 8} \pm \mathbf{0 . 2}$ \\
\hline $\begin{array}{c}\text { along } \\
\text { scanline }\end{array}$ & $8-10$ & 1 & 0.5 & 0.8 & 0.4 & 0.7 & 3.8 \\
\hline
\end{tabular}




\section{Statistical Methods of Fracture Characterisation Based on Acoustic Borehole Televiewer Log Analysis}

\subsection{Abstract}

Acoustic Borehole televiewer (BHTV) logs provide measurements of fracture attributes (orientations, thickness, spacing) in boreholes. Orientation, censoring and truncation sampling biases similar to those described for 1-dimensional outcrop scanlines, and other logging or drilling artefacts specific to BHTV logs, can affect the interpretation of fracture attributes from BHTV logs. K-means, fuzzy K-means, and agglomerative clustering methods provide transparent means of separating fracture groups on the basis of their orientation. Fracture spacing is calculated for each of theses fracture sets. Maximum likelihood estimation using truncated distributions permits the fitting of several probability distributions to the fracture attribute datasets within truncation limits, which can then be extrapolated over the entire range where they naturally occur. Akaike Information Criterion (AIC) and Schwartz Bayesian Criterion (SBC) statistical information criteria rank the distributions by how well they fit the data. We demonstrate these attribute analysis methods with a dataset derived from three BHTV logs acquired from the high-temperature Rotokawa Geothermal Field, New Zealand. Even for the spacing analysis spanning three orders of magnitude, several distributions have similar AIC rankings for datasets of up to 300 data points. Varying BHTV log quality reduces the number of input data point, but careful selection of the quality levels where fractures are deemed fully sampled increases the reliability of the analysis. Several clustering configurations and probability distributions can often characterise the data at similar levels of statistical criteria. Thus, several scenarios should be considered when using BHTV log data to constrain numerical fracture models.

\subsection{Introduction}

Defining the geometries of fracture systems in reservoirs requires a robust delineation of individual fracture sets of similar orientation and of their geometrical variability. Each fracture set can result from a specific geological event and/or a different process 
(Bonnet et al., 2001; Dezayes et al., 2015). Different fracture sets can have different geometrical (e.g., size, aperture and density) and mineralogical (infill material and quantity) properties, and thus may have different impacts on the hydrology of the system (Agosta et al. 2010). In addition, numerical models of fracture systems and their associated fluid pathways in reservoirs can be constrained by the assumed probability distributions of aperture, length, and density of each fracture set (e.g., Dershowitz and Einstein, 1988; Bonneau et al., 2013), which thus need to be assessed carefully.

The classical method for delineating fracture sets is to plot fracture orientations on hemispherical projections (stereonets) and contour the projected poles to fracture planes by their areal density, yielding a "Fisher" density. This method is intuitive and straightforward to apply thanks to stereonet software packages. However, the delineation of fracture sets from these contours is subjective and may vary between data analysts. Cluster analysis of fracture orientation provides transparent means of separating fracture orientation data into sets, as proposed by Shanley and Mahtab (1976). Agglomerative, K-means, and fuzzy K-means clustering have been used to analyse orientation data derived from scanline measurements, predominantly for engineering purposes (e.g., Hammah and Curran, 1998, 1999, Zhou and Maerz, 2002; Tokhmechi et al., 2011; Li et al., 2014b).

Acoustic borehole televiewer (BHTV) logs provide an image of the inside of a borehole by measuring the acoustic amplitude and return time of an ultrasonic signal emitted by the logging tool (Zemanek et al., 1970, Poppelreiter et al., 2010). Analysis of these logs yields continuous samples of fractures intersecting the borehole, including their position, orientation, and thickness. In general, BHTV logs cannot discriminate between open and closed fractures (unless the fractures are infilled with high acoustic impedance minerals such as quartz; Milloy et al., 2015), so the fracture aperture and thickness are both referred to without discrimination as "thickness" in this chapter. Image logs, such as BHTV logs, are particularly useful in boreholes where continuous oriented drill-cores are not available, as is commonly the case in hydrocarbon and geothermal reservoirs. Image logs are subject to similar sampling biases to 1dimensional (1-D) outcrop scanlines, as well as biases related to drilling and logging artefacts (e.g., Lofts and Bourke, 1999) that can decrease image quality in places. All these biases need to be evaluated and incorporated into the statistical analysis of fracture geometries.

Fracture attributes (e.g., thickness, spacing, length) have been shown to variously follow power-law, exponential, gamma, log-normal and power-exponential distributions (see Bonnet et al., 2001; Torabi and Berg, 2011, and references therein). Faults commonly exhibit power-law spacings, although this may partially reflect sampling bias and the mixing of different fracture sets (Gillespie et al., 1993; Nicol et al., 1996). Power-law exponents are commonly estimated using linear regression to the cumulative density function (CDF) of observations in doubly logarithmic axes ("log-log" plots; 
see McCaffrey et al. (2003) for example). However, this method is not mathematically robust, and does not unambiguously demonstrate that the observations are power-law (Clauset et al. 2009). The maximum likelihood estimation (MLE) method is preferred to least-square fitting for estimating which probability distribution and associated parameters best fit a set of data (Laslett, 1982; Villaescusa and Brown, 1992; Clauset et al., 2009). The MLE method estimates values for the parameters of a given distribution that maximises a likelihood function, i.e. finds the parameter set that makes the observed data most likely.

This chapter presents methods with which to discriminate between fracture sets of different orientation using clustering algorithms, and to evaluate the probability distribution and parameters of fracture attributes (thickness, spacing) via maximum likelihood estimations. Fractures used as examples are interpreted from three BHTV logs acquired in the high-temperature Rotokawa Geothermal Field, Taupo Volcanic Zone, New Zealand. Our analysis includes consideration of sampling biases in BHTV logs, a strategy for accommodating varying BHTV log quality, and a qualitative estimation of the size and range of dataset to constrain the distribution fitting well.

\subsection{Data}

The fracture dataset utilised in this chapter was derived from three BHTV logs acquired in boreholes RK18L2, RK30L1 and RK32 in the high-temperature Rotokawa Geothermal Field, located in the Taupo Volcanic Zone, New Zealand. These data were described by McNamara et al. (2015) and have been further improved for a more accurate measurement of fracture thickness. The BHTV logs were acquired with the ABI-85, a high-temperature logging tool able to operate at up to $300^{\circ} \mathrm{C}$ Ásmundsson et al., 2014). The boreholes intersect several lithologies; here, we focus on the andesitic formations which form the main part of the deep reservoir McNamara et al. 2016a). Fractures are dominantly steeply-dipping ( $>60^{\circ}$ dip) and striking parallel to the azimuth of maximal horizontal compressive stress $\left(\mathrm{S}_{\mathrm{Hmax}}\right)$ which varies between NNE-SSW and ENE-WSW, and reflects the regional normal faulting in the Taupo Rift (McNamara et al., 2015, 2016a).

Fractures were identified in the BHTV logs using the Recall ${ }^{T M} 5.3$ software following the methods discussed by Massiot et al. (2015). Borehole deviations of up to $30^{\circ}$ from vertical were accounted for during the log processing, with the orientations presented here referenced to geographic north and horizontal. In total, 1218 fractures were identified over $2350 \mathrm{~m}$ of BHTV logs (Figure 3.1); they are described in terms of their location along the borehole, orientation, thickness, and descriptive characteristics (e.g., high or low acoustic amplitude, and cross-cutting relationships with nearby fractures). The apparent fracture thickness measured at the borehole wall has been converted to 


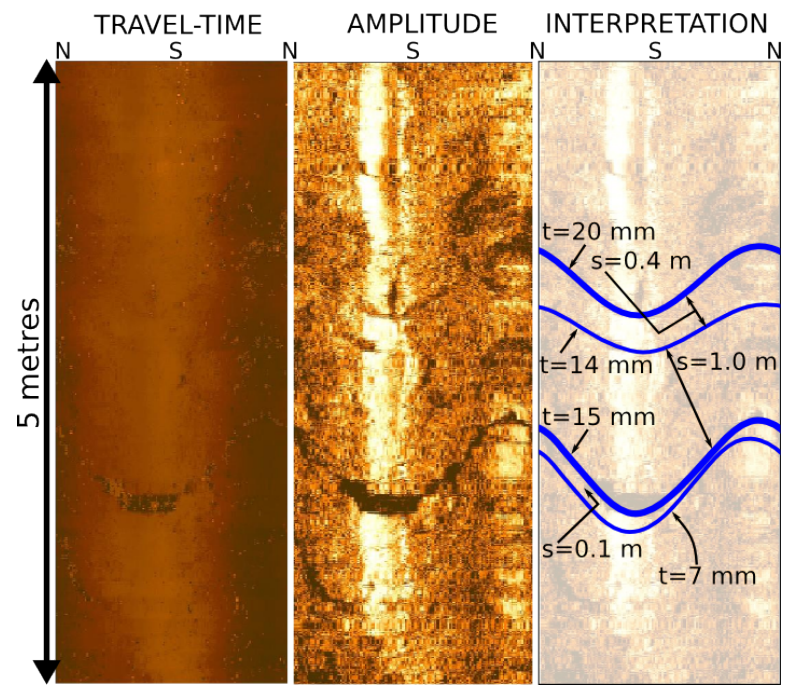

Figure 3.1: Example of a BHTV log with unwrapped travel-time and amplitude oriented images showing various fracture thickness ("t") and spacing ("s") values.

the true thickness that would be measured perpendicular to the fracture walls (Barton and Zoback, 1992; Massiot et al., 2015). The apparent spacing between neighbouring fractures of the same set measured along the borehole trajectory has been converted to the fracture-normal spacing (Priest, 1993$)$.

The BHTV logs acquired in the Rotokawa reservoir commonly have only partial coverage of the borehole and contain a number of logging- and drilling-induced artefacts that decrease the image quality (Lofts and Bourke, 1999; McNamara et al., 2015). Image quality has been qualitatively assigned using a five-class ranking scheme (Massiot et al. , 2015). Fracture detection can be hindered in zones of low- or very poor-quality images, which may adversely affect the statistical analysis of fracture attributes, particularly the spacing. Orientation analysis has been performed for each borehole individually and the data combined, after correction for orientation sampling bias Terzaghi, 1965; Massiot et al. 2015). Other biases associated with incomplete detection of small attribute values, and the low probability of occurrence of high-attribute values, will be specifically addressed in the chapter.

\subsection{Fracture Set Orientation Clustering}

\subsubsection{Cluster Analysis Methods}

Fracture sets are commonly identified on stereonets using Fisher or Kamb contours, but discrimination between fracture sets is not always clear where high-density zones overlap. Clustering algorithms divide a dataset into groups (clusters) of observations that are similar to each other. In such algorithms, a "dissimilarity" metric must be selected to describe how far apart data points are from each other. The metric summarises 
all relevant aspects of similarity into a single measure. Here, we use the acute angle between the normals of each pair of fractures as the dissimilarity metric for fracture orientations. Two sub-parallel, steeply dipping $\left(>80^{\circ}\right)$ fractures may have different dip directions $\left( \pm 180^{\circ}\right)$ but small acute angle between their normals, and may thus belong to the same set. Two types of cluster analysis are used in this work: partitional, based on a prior specification of the desired number of clusters $(\mathrm{K})$; and hierarchical, which constructs a hierarchy between one and $n$ nested clusters, $n$ being the number of observations.

K-means clustering is a partitional method that assigns each data point to one of $K$ clusters (James et al. 2013$)$. The assignment is an iterative process that minimizes a sum of dissimilarities between the centroid of the cluster and each data point within the cluster. Data points are re-allocated between clusters until an optimal configuration is reached. This process is computationally efficient, but issues result from the initialisation of the initial centroid locations, which can lead to the non-unique selection of a local minimum instead of the global minimum of the sum of dissimilarities, and the possible generation of empty clusters (James et al. 2013$)$. Comparing clustering results using different initial centroids mitigates these risks. The K-means method is most successful when applied to globular clusters of similar sizes and densities, without too many outliers, and to find sub-clusters. Defining the optimal number of clusters for a dataset is non-trivial. Good clusterings have a low cohesion, where objects are close to each other within their cluster; and a high separation, where clusters are distinct from each other (Figure $3.2 \mathrm{~b}$ ). The silhouette value combines the cohesion and separation values (Reynolds et al., 2006). For each data point $i$, the silhouette value is calculated as:

$$
s(i)=\frac{b(i)-a(i)}{\max (a(i), b(i))}
$$

where a(i) is the average dissimilarity of the data point $i$ with all other data within the same cluster (cohesion), and $b(i)$ is the lowest average dissimilarity of $i$ to any cluster of which the $i^{\text {th }}$ datum is not a member (separation). A value of $s(i)$ close to 1 indicates that the datum is appropriately clustered. When testing several input numbers of clusters, the optimal cluster number is found when the average s(i) for the entire dataset is maximised (Figure 3.2 a).

The fuzzy K-means approach allows the data points to belong to several clusters at once, with varying degrees of membership to each cluster, which is appropriate when clusters are overlapping or not compact (Zadeh, 1965). For example, a data point can belong $20 \%$ to one cluster and $80 \%$ to a second cluster; overall, the data point belongs predominantly to the latter cluster (Figure $3.2 \mathrm{~d}$ ). A clustering result that maximises the membership level for each data point to their dominant cluster is thus desirable, and is achieved here by calculating the median of each data point's highest membership level ( $80 \%$ for the data point in this example). A high median membership indicates a 
a)

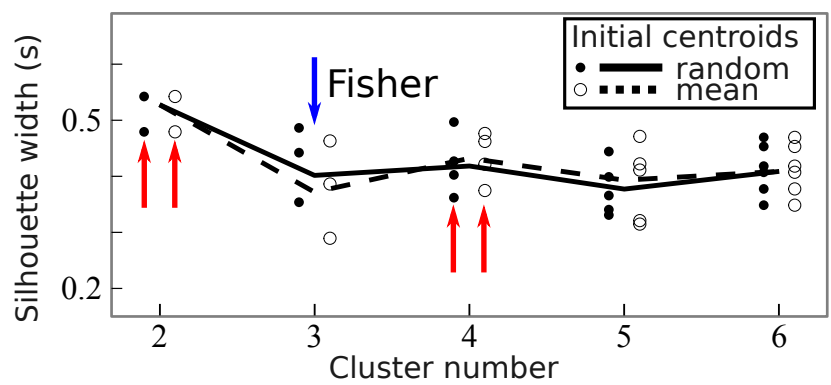

c)

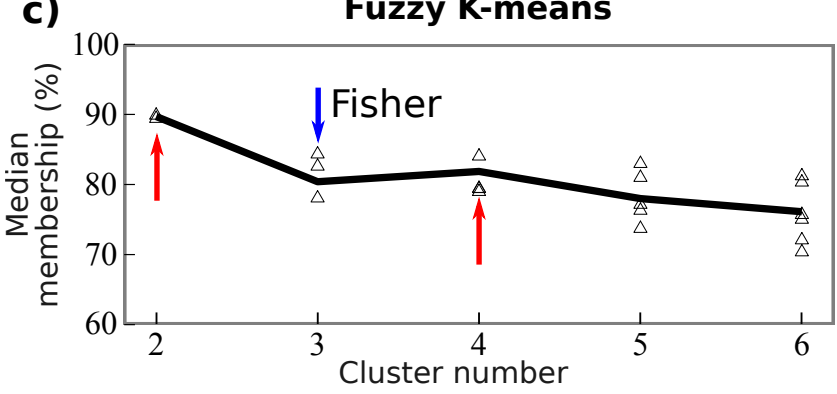

e)

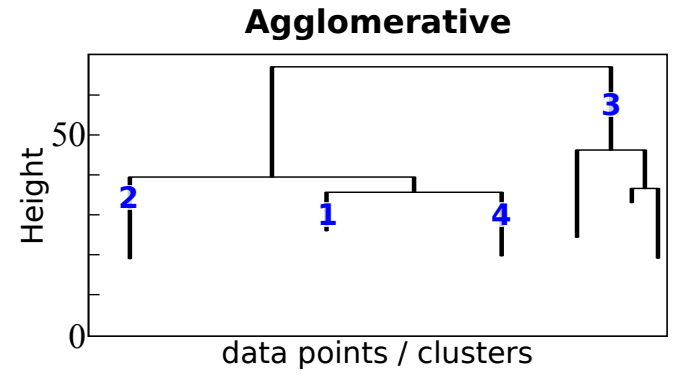

b)

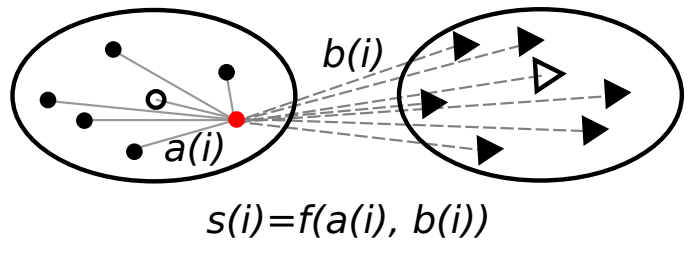

d)

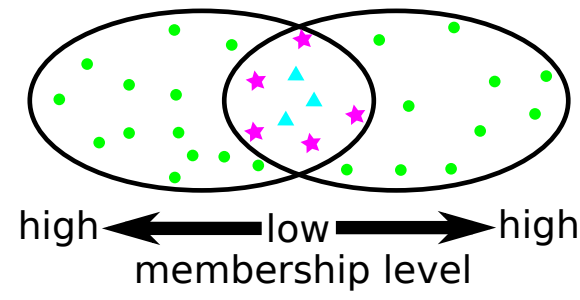

f)

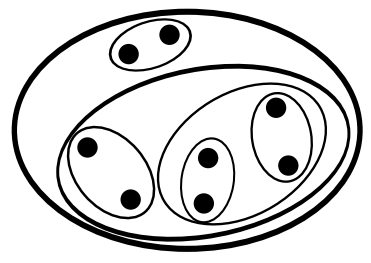

Figure 3.2: Evaluation of the optimal number of clusters for borehole RK30L1. The best clusterings are indicated by the underlying arrows, and the configuration interpreted from Fisher contours by overlying arrows. a) K-means: average silhouette width for each cluster (dots), and average of the average silhouette widths (line) for each number of clusters, with either random initial centroids, or located at the mean orientation of the fracture set. b) Synthetic K-means clustering with silhouette $s(i)$ calculation as a function of cohesion $a(i)$ and separation $b(i)$; the centroids of each clusters are open symbols. c) Fuzzy K-means: median membership level for each cluster (triangles), and overall median membership level (line) for each number of clusters. d) Synthetic fuzzy K-means of overlapping clusters with varying membership levels. e) Dendogram of the agglomerative clustering, with the levels at which each of the four main fracture sets emerge. f) Synthetic representation of successive agglomeration of data points or clusters. Evaluation plots for other boreholes and combined is in Appendix 3.A. 
well-separated clustering result (Figure 3.2 c).

The agglomerative hierarchical clustering method, also called bottom-up, starts with each observation constituting a cluster, and successively merges the two nearest clusters until only one large cluster containing all the observations remains James et al., 2013, Figure 3.2 $\mathrm{f}$ ). Thus, each step $j$ of the agglomerative process contains $(n-j+1)$ clusters. In contrast with the K-means clustering, agglomerative hierarchical clustering is not affected by the initialisation step and local minima. However, the merges are definitive and the process can produce unstable clusters when data points are allocated early to a cluster that they would not belong to if they had been allocated later. Agglomerative clustering is also more computationally expensive than the K-means process. The sequence of agglomerative clustering can be visualised on a dendogram, a graphical representation of the nested clusters (Figure 3.2 ). It represents the successive grouping of sub-clusters until the entire dataset belongs to a single cluster: observations that are grouped early, near the bottom of the dendogram, are similar to each other, whereas observations that fuse later (close to the top) are more distinct. A single dendogram can be used to obtain the partitioning of the dataset in any desired number of clusters.

\subsubsection{Fracture Set Delineation Example}

To estimate the number of clusters best characterising the datasets, and to assign each data point to a cluster, the fracture orientations are first analysed using Fisher density contours on a stereonet (presented for borehole RK30L1 in Figure 3.3a). The loci of poles to under-sampled fracture planes sub-parallel to the borehole are plotted as a shaded series of great circles. The fracture orientations are then clustered using Kmeans, fuzzy K-means and agglomerative methods, for 2 to 6 clusters using the pam, fanny and agnes functions in $R$, respectively $(R$ Core Team, 2015). Outliers, defined as having $<1 \%$ Fisher density, were removed from the dataset prior to the cluster analysis. As suggested by K-means theory (James et al., 2013$)$, tests run with outliers did not provide satisfying results. In such cases, both gently $\left(<40^{\circ}\right)$ and steeply $\left(>70^{\circ}\right)$ dipping fractures of similar dip direction were grouped in the same cluster, a result inconsistent with both Fisher contours analysis and the normal faulting regime at Rotokawa, where steeply dipping fractures are associated with normal faults.

Each K-means clustering is made with two types of initial centroids: (1) imposed initial centroids located at the mean orientation of the fracture sets defined from Fisher contours (Figure 3.3b); and (2) randomly allocated (Figure 3.3p). Fuzzy K-means clustering analysis highlights which fractures tend to lie between clusters (Figure $3.3 \mathrm{~d}$ ). The dendogram of the agglomerative clustering is presented in Figure 3.2 for the six highest-level clusters, i.e. corresponding to between 2 and 6 clusters. The lower part of the diagram, which includes all input data points, has been removed for clarity. 
a) Fisher
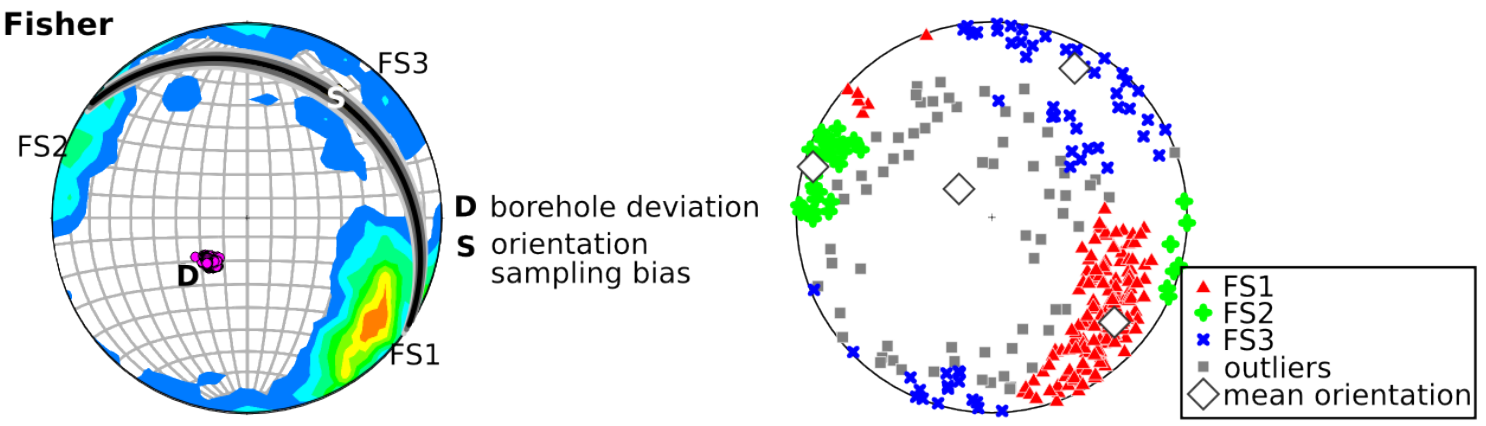

b) K-means $\quad K=2$

$\mathrm{K}=3$
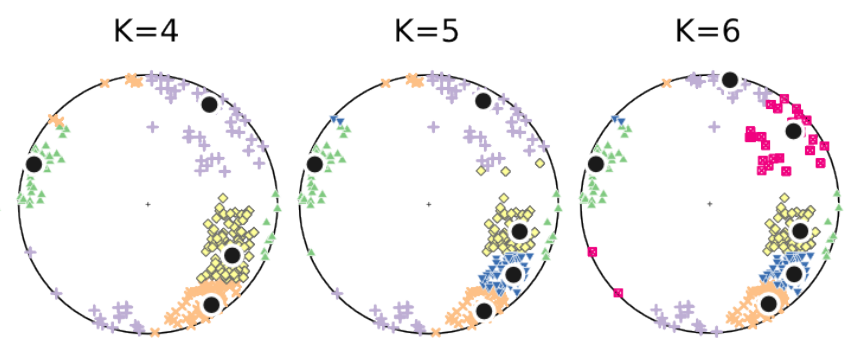

c)
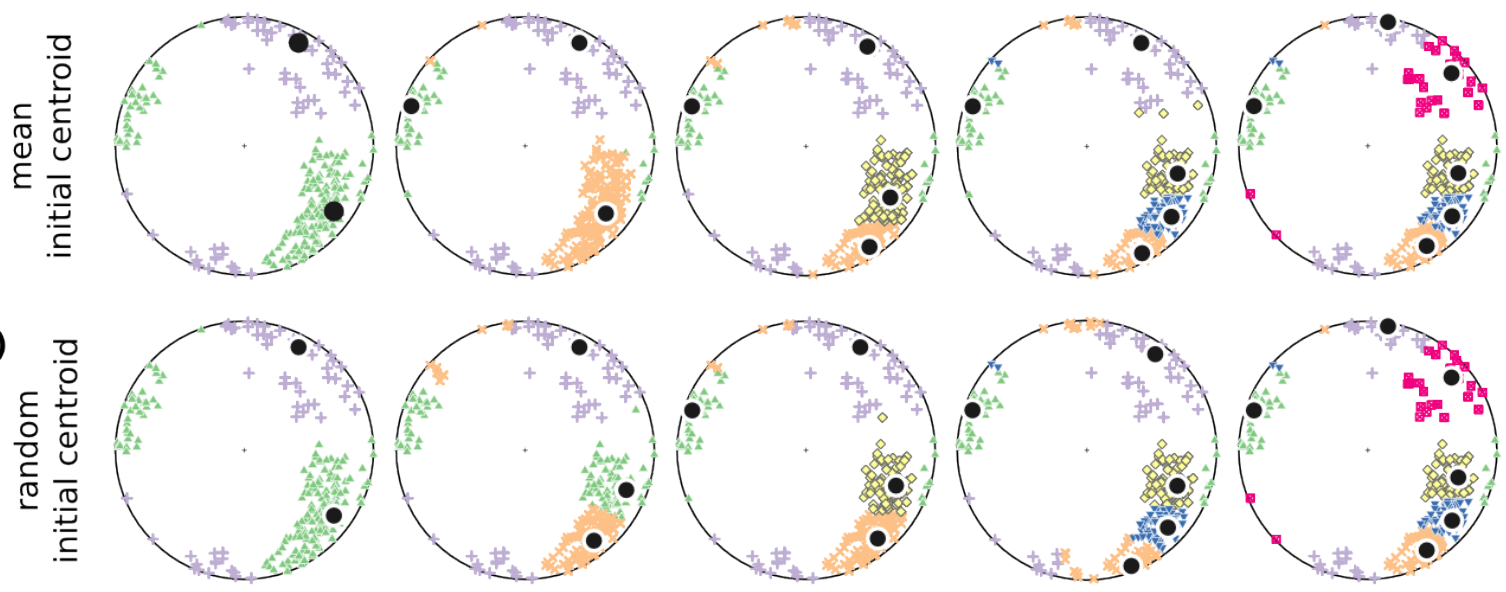

\section{d) Fuzzy K-means}

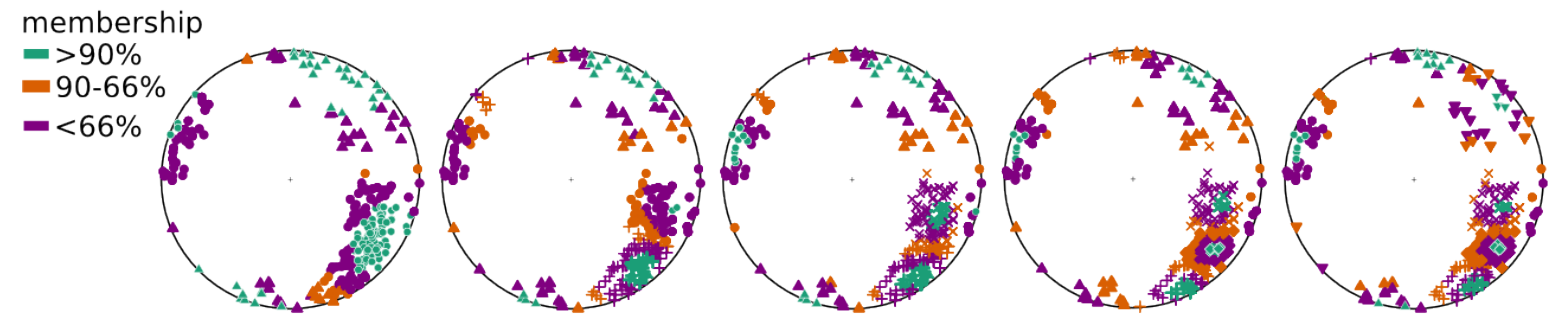

\section{e) Agglomerative}

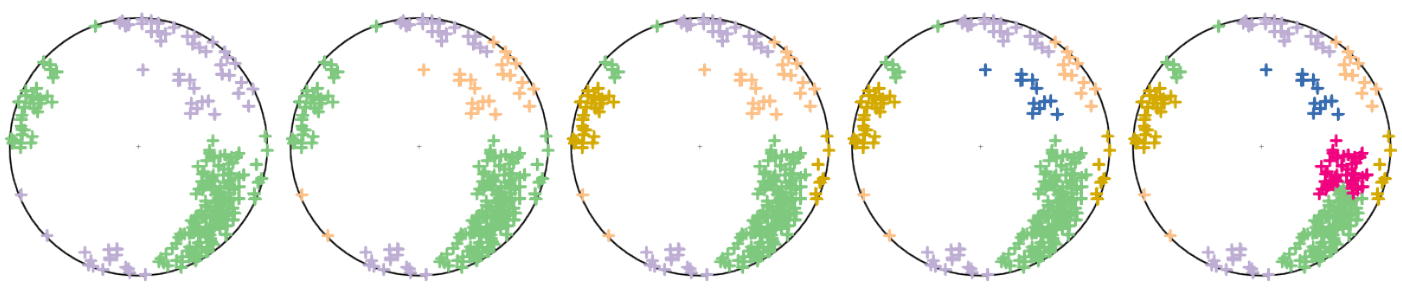

Figure 3.3: Example of fracture orientation analysis for borehole RK30L1. a) Fisher contour, and pole to planes with the resulting delineation comprising three fracture sets and outliers. b) K-means clusters with the initial centroid specified at the mean orientation of the fracture sets, with 2 to 6 clusters $(\mathrm{K})$. The centroid of each cluster is noted as a black dot. c) K-means clusters with random initial centroids. d) Fuzzy Kmeans clustering; colours indicate the membership level (input membership component is 1.5). e) Agglomerative clustering. The colours in b), c) and e) are arbitrarily affected to each cluster. 
For configurations with two clusters, the results of K-means and agglomerative clusterings are similar to the sets defined from Fisher contours (see Figures 3.2 and 3.3 for example in borehole RK30L1; results for other boreholes are in Appendix 3.A. With more than two clusters, the K-means and agglomerative clusterings yield different results. The optimal number of clusters for each dataset is selected based on the highest average silhouette width of the K-means clustering, and highest median membership levels of the fuzzy K-means clustering (Figures $3.2 \mathrm{a}$, c). For borehole RK30L1, the configurations with two and four clusters are the best clusterings, contrary to the three-cluster allocation interpreted from Fisher contours. The decrease in median membership for configurations with more than four clusters suggests that configurations with more clusters are unlikely. In addition, the statistical analysis of fracture attributes requires a sufficient number of fractures per set (see Section 3.5.5), which is not the case for configurations with more than four clusters and thus not desirable.

The choice of initial centroids of the K-means algorithm influences the clustering results for individual boreholes, but not for the combined dataset containing more data points (Appendix 3.A. Figure 3.A.1). Tests performed with several sets of randomly selected initial centroids yielded similar results. Random initial centroids are preferred to the imposed initial centroids, because they produce similar or higher mean silhouette widths than when the initial centroids are imposed at the mean orientation of fracture sets.

The dendogram of the agglomerative clustering provides additional insights into how well the clusters are separated, and potential sub-clusters within the four fracture sets identified in this study. In borehole RK30L1, fracture set 3 is distinct from the combination of fracture sets 1,2 , and 4 , as they are the last groups to be agglomerated; fracture sets 1 and 4, agglomerated early, are quite similar and may form sub-clusters (Figure 3.2 ).

\subsection{Probability Distribution Analysis of Fracture Attributes}

\subsubsection{Sampling Biases}

As in the case with 1-D scanline outcrop measurements, fracture data interpreted from BHTV logs are subject to resolution limits and sampling biases Laslett, 1982; Barton and Zoback, 1992; Pickering et al., 1995; Nicol et al., 1996). Small fracture thickness and spacing values are only partially recorded close to the resolution limit of the dataset ("left-hand truncation"); conversely, high values may not be recorded because they are rare and may exceed the capacity of the measuring technique used to record 

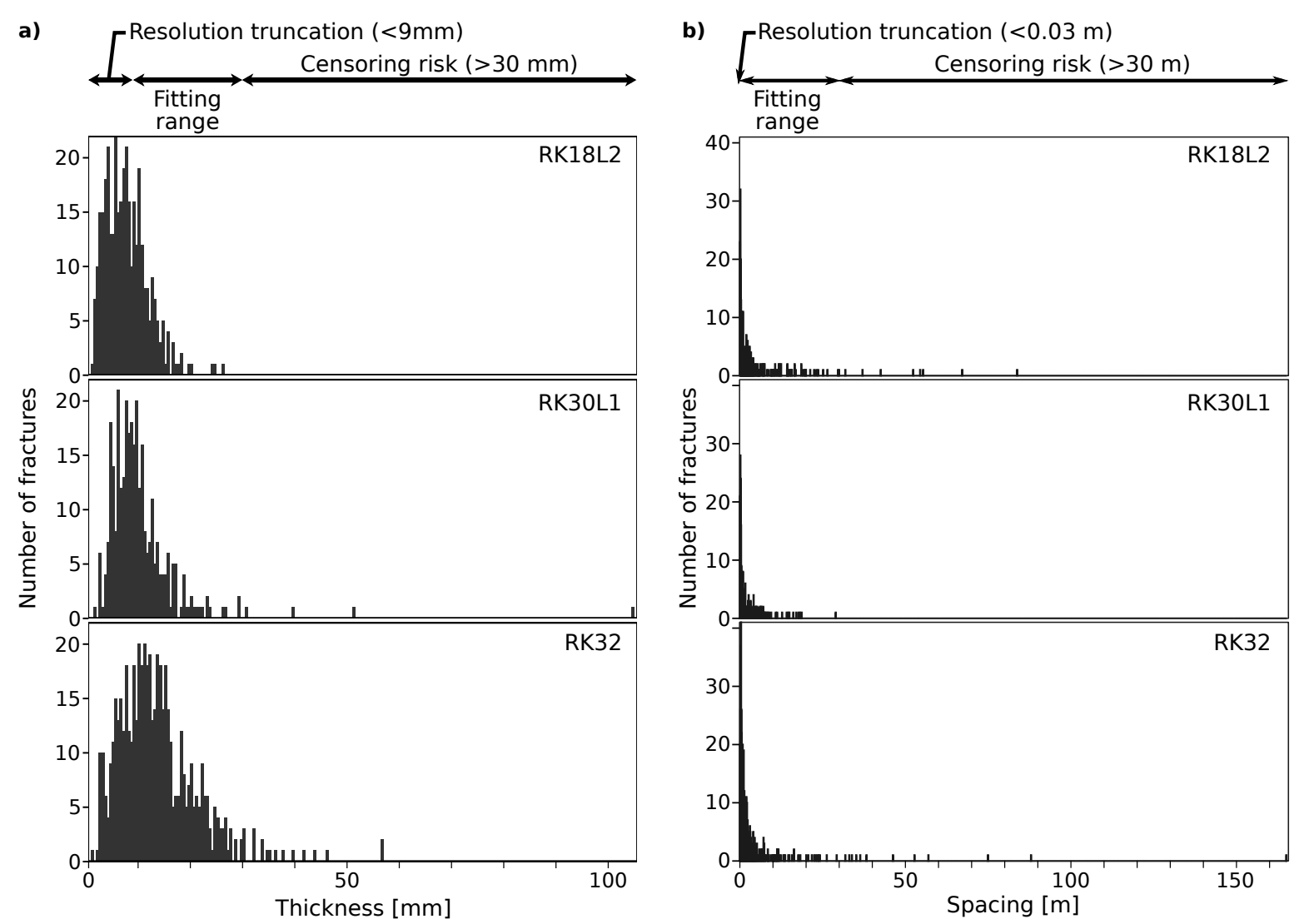

Figure 3.4: Histogram and sampling bias limits for fracture thickness (a) and spacing (b) for wells RK18L2, RK30L1 and RK32. Fracture spacing values from each fracture sets are superimposed in b).

them ("right-hand truncation"). The probability distribution of fracture attributes measured on the BHTV logs is thus estimated by fitting truncated distributions between truncation limits (scale range) within which all fractures are deemed to have been sampled. Truncated distributions are conditional distributions that result from restricting the domain of validity of a probability density function. The resulting distribution form and parameters fitted to a truncated dataset can then be extrapolated to estimate the distribution of values beyond truncation limits.

In the Rotokawa reservoir, fractures thinner than the BHTV log resolution of $\sim 5 \mathrm{~mm}$ cannot be detected, except in rare cases where there is a large acoustic impedance contrast between the fracture and the host lithology. Histograms of fracture thickness indicate a paucity of fractures less than $8 \mathrm{~mm}$-thick in boreholes RK18L2 and RK30L1 and less than $9 \mathrm{~mm}$ in borehole RK32 (Figure 3.4a). Therefore, $9 \mathrm{~mm}$ is used as the left-hand truncation limit for fracture thickness. The right-hand truncation limit in BHTV logs is difficult to determine because it arises from a paucity of thick fractures rather than their incomplete detection by the tool. In addition, it is possible that rare fractures exceeding $30 \mathrm{~mm}$ thickness comprise several closely-spaced fractures not resolved individually on the BHTV $\log$ ("censoring"). Zuquim and Rowland (2013) reported that extensional fractures in a paleo-hydrothermal system hosted in lava flows 
in the Coromandel area, north of the Taupo Volcanic Zone, were typically up to $30 \mathrm{~mm}$ thick, with some fractures up to a few hundreds of millimetres thick. Similarly, analysis of 3000 fractures in continuous cores from the Soultz geothermal reservoir found the thickest fracture to be $250 \mathrm{~mm}$ thick (a quartz vein), but that fractures exceeding $10 \mathrm{~mm}$ in thickness were rare (Genter and Traineau, 1996). Overall, we consider the 9-30 $\mathrm{mm}$ range of fracture thickness to be fully sampled in the BHTV logs acquired in the Rotokawa reservoir; this range is analogous to that used in a similar study at Cajon Pass, U.S.A. (Barton and Zoback, 1992).

The left-hand truncation limit for the fracture spacing analysis is $30 \mathrm{~mm}$, based on the odds of closely-spaced fractures over intervals $<30 \mathrm{~mm}$ not being resolved by the BHTV log, and the right-hand limit is set at $30 \mathrm{~m}$ due to the scarcity of fractures with larger spacings (Figure $3.4 \mathrm{~b}$ ).

\subsubsection{Effects of BHTV Log Quality}

We consider the BHTV logs to sample most fractures in zones of quality ranking $\geq 3$. Indeed, the small number of fractures in the two lowest-quality ranking zones $(1,2)$ is interpreted to be due to incomplete sampling of fractures, and analysis of fracture spacing within these zones is thus inappropriate. The fracture detection is best in zones of high quality rankings $(4,5)$, but these zones are mostly $<20 \mathrm{~m}$ long, preventing the evaluation of large spacings (i.e., $>10 \mathrm{~m}$ ). In addition, there can be too few data points in zones of high quality ranking (4-5) for statistical analysis (14 points in zones of ranking 4-5, compared to 118 in zones of ranking 3-5 for fracture set 2 in borehole RK18L2; Figure 3.5b). The cumulative density functions (CDFs) of fracture spacing in zones of high quality rankings (4-5) are similar to those of moderate quality ranking quality (3-5; Figure 3.5). As a result, statistical analyses of fracture spacing in zones of moderate quality will provide similar results to those made in zones of high quality, but with higher confidence in the results as there are more data points.

In the Rotokawa Geothermal Field, where $50 \%$ of the BHTV logs are of intermediate quality (rank 3), the statistical analysis of fracture spacing in zones of ranks $3-5$ thus represents a good compromise between (1) a sufficient log quality allowing the sampling of most of the fractures that can be detected by the BHTV log and (2) sufficient length of continuous sections of log to sample large spacings.

\subsubsection{Maximum Likelihood Estimation Using Truncated Dis- tributions}

Five probability distributions are commonly used to describe fracture attributes such as thickness and spacing: exponential, gamma, log-normal, power-law and powerexponential (Table 3.B.1; Gillespie et al. (1993); Bonnet et al. (2001) and references 

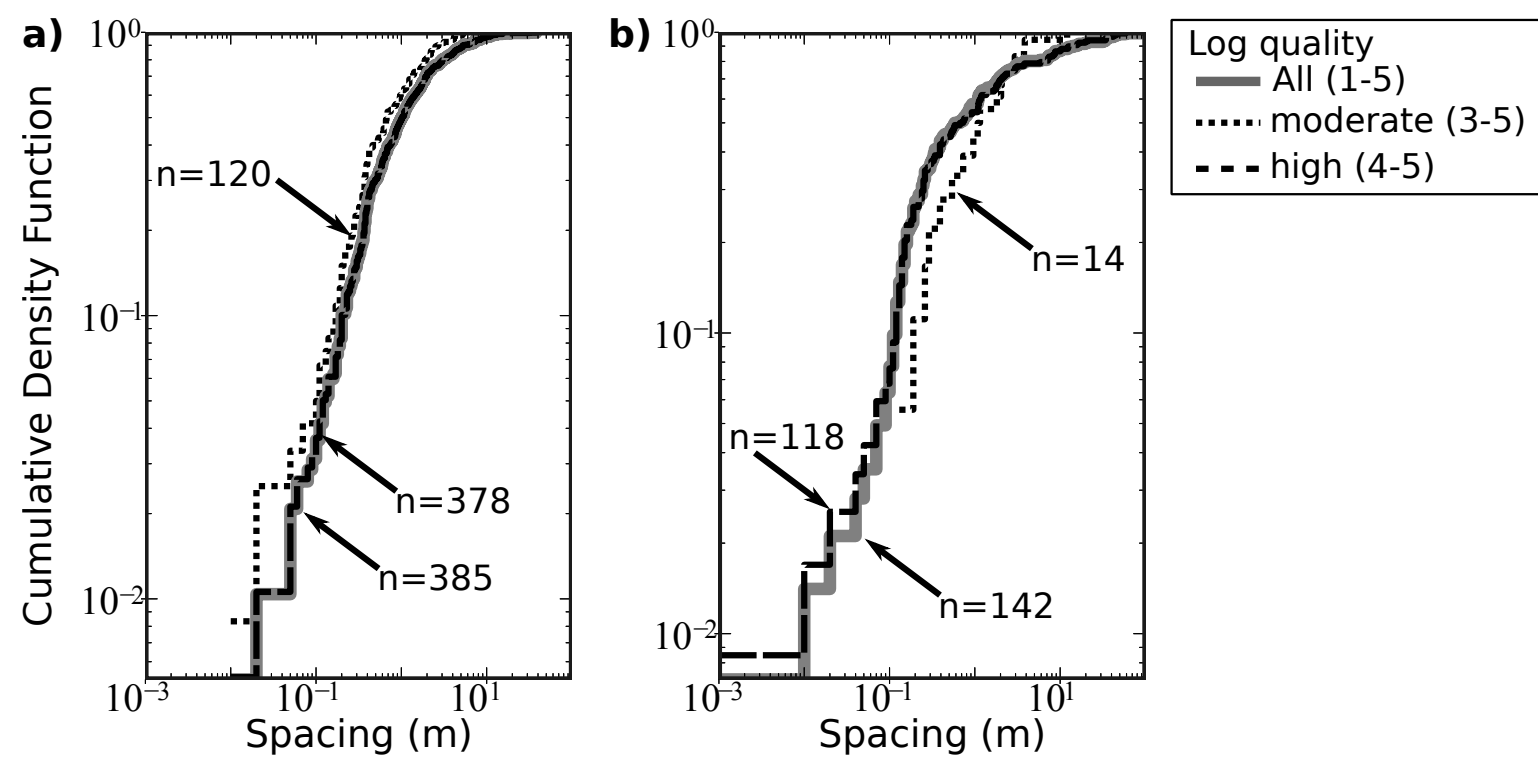

Figure 3.5: Cumulative density functions of fracture spacing with fractures in all BHTV log quality zones (rank 1-5), in moderate quality zones (rank 3-5), and in high BHTV log quality zones only (rank 4-5). a) Fracture set 1 in borehole RK32 (the curves for all and moderate quality ranks plot on top of each other). b) Fracture set 2 in borehole RK18L2. $n$ : number of data points in each subset.

therein). All these distributions have characteristic scales, except for the power-law which is scale-invariant.

Probability distributions are fitted to the fracture thickness and spacing data interpreted from BHTV logs using the Generalized Additive Models for Location Scale and Shape library (GAMLSS; Stasinopoulos and Rigby, 2007, Table 3.B.1). This statistical modelling framework implemented in $R$ uses the maximum likelihood estimation (MLE) method, which provides robust estimators by maximizing the likelihood function of the considered distribution (Newman, 2005; Guerriero et al., 2011). GAMLSS can be used to evaluate numerous distributions, which can all be truncated. The powerlaw distribution is evaluated in GAMLSS with a pareto type II distribution. Contrary to a power-law distribution, the pareto distribution does not appear as a straight line on log-log plots, but its form and coefficients are directly related to a power-law under certain conditions (satisfied for thickness and spacing in the Rotokawa reservoir; Table 3.B.1). The power-law exponent is also fitted by MLE independently from GAMLSS Clauset et al. 2009, and references therein; Table 3.B.1). For reference, the power-law exponent is also evaluated with a least-square regression on log-log plots, although this is not mathematically robust (Pickering et al., 1995; Newman, 2005).

Given a set of distributions fitted to a dataset, the best-fitting distribution amongst the set of distributions investigated is the one with the lowest Akaike Information Criterion (AIC; Akaike, 1974), or Schwarz Bayesian Criterion (SBC; Schwarz, 1978). The AIC and SBC values are derived from the generalised Akaike information criterion 
(GAIC) for each tested distribution:

$$
G A I C=-2 \hat{\ell}+p \cdot d_{f}
$$

where $\hat{\ell}$ is the maximised value of the log-likelihood function, $p$ is a penalty factor $(p=2$ for AIC and $p=\ln (n)$ for SBC, where $n$ is the number of observations), and $d_{f}$ is the number of degrees of freedom (number of free parameters of the studied distribution). The AIC and SBC are thus measures of the trade-off between closeness of fitting (higher $\hat{\ell}$ ) and complexity (larger number of parameters). Distribution selection with the SBC, which has a more severe penalty for increasing the degrees of freedom, results in a simpler model (i.e. fewer parameters) than that selected by AIC. The use of the SBC for selecting models prevents model overfitting, i.e. when the number of parameters is too large relative to number of observation. At equal maximised log-likelihood $\hat{\ell}$ amongst the five tested distributions, the exponential distribution is thus favoured by the AIC (and even more by the SBC) criterion as it has only one parameter, and the power-exponential which has three parameters is the most penalised (also even more penalised by the SBC). The difference $\left(\Delta\left(A I C_{k}\right)=A I C_{k}-A I C_{\text {min }}\right)$ between the lowest $\mathrm{AIC}\left(\mathrm{AIC}_{\text {min }}\right)$ and the $\mathrm{AIC}_{k}$ values of an alternative distribution $k$ increases as the goodness of fit of the distribution $k$ decreases. We considered that models with $\Delta\left(A I C_{k}\right)<5$ have substantial support (Burnham and Anderson, 2002).

The histogram $h^{n}(x \mid \Theta)$ of $n$ values having a probability distribution $f(x \mid \Theta)$ is: $h^{n}(x \mid \Theta)=n \cdot f(x \mid \Theta)$. For distributions evaluated within a truncated range, the extrapolation of the histogram out of truncation bounds is calculated with:

$$
h^{N_{t o t}}(x \mid \hat{\Theta})=\frac{N_{t r}}{F\left(x_{2} \mid \hat{\Theta}\right)-F\left(x_{1} \mid \hat{\Theta}\right)} f_{t r}(x \mid \hat{\Theta})=N_{t o t} f_{t r}(x \mid \hat{\Theta})
$$

where $f_{t r}(x \mid \hat{\Theta})$ is the probability density function estimated within truncated range with a series of estimated parameters $\hat{\Theta} ; N_{t o t}$ is the total number of fractures assuming there were no truncations, calculated from the number of samples occurring within the truncated range $\left(N_{t r}\right)$ and the cumulative density function $(\mathrm{CDF})$ of the non-truncated distribution $F(x \mid \hat{\Theta})$ evaluated on the lower $\left(x_{1}\right)$ and upper $\left(x_{2}\right)$ truncation limits. The functions $f$ and $f_{t r}$ have the same form and parameters.

\subsubsection{Distribution Fitting Example}

Figure 3.6a shows an example of distribution fitting to the spacing of the dominant fracture set ("1") in all three boreholes combined. In this case, the log-normal distribution fits the data best out of the five distributions considered, and there are no other close contenders $\left(\Delta\left(A I C_{i}\right)\right.$ and $\Delta\left(S B C_{i}\right)>5$ for all other distributions $)$. Visually, the exponential and gamma distributions do not fit well, but it would not be possible to 

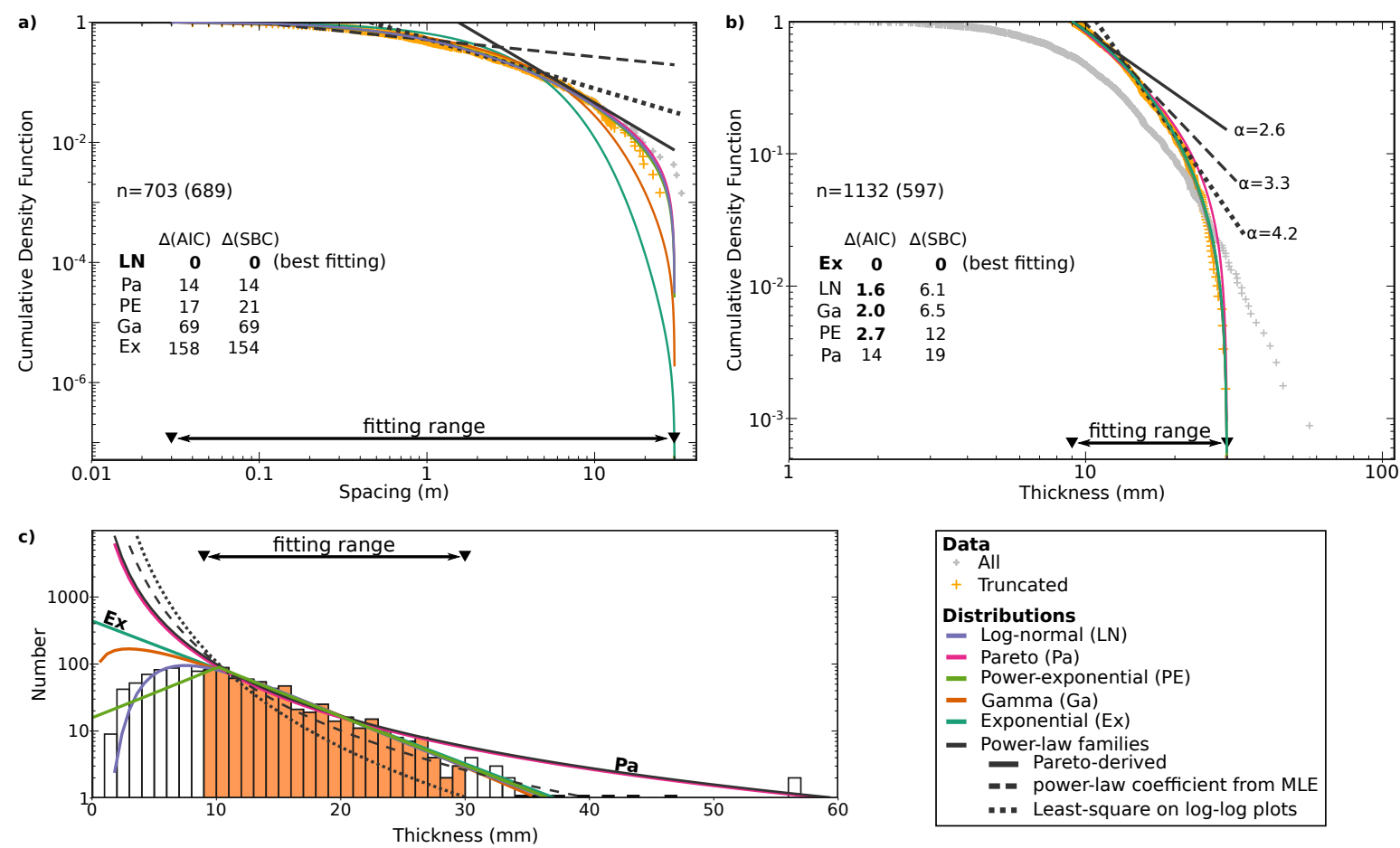

Figure 3.6: Estimations of the probability distribution of fracture attributes. a) CDF of the spacing data of fracture set 1 in all three boreholes combined, and fitted distributions. b) CDF of fracture thickness data for all fractures, and fitted distributions. $n$ : total number of fractures in the dataset, truncated number within brackets. $\Delta(\mathrm{AIC})$ and $\Delta(\mathrm{SBC})$ are indicated for all distributions, with those distributions satisfying $\Delta(\mathrm{AIC})$ or $\Delta(\mathrm{SBC})<5$ in bold. c) Histogram of all measured fracture thickness in log-linear scale, with the probability density functions of distributions fitted over the $9-30 \mathrm{~mm}$ scale range, extrapolated over the entire range of observation. In this coordinate system, the exponential distribution plots as a straight line.

rule out the pareto and power-exponential distributions. None of the power-law-type distributions fit the data well, and provide coefficients of 1.3 (MLE estimation of powerlaw coefficient), 1.8 (least-square regression in log-log plot) and 2.6 (derived from the pareto distribution), which is a wide range that encompasses most values found in geological systems (1 to 3 ; Bonnet et al., 2001).

In some cases, as with the fitting of the fracture thickness, several distributions yield similarly good fits (Figure 3.6b): the exponential distribution has the best fit $\left(A I C=A I C_{\min }\right)$ but all other distributions with the exception of pareto are just as good according to the AIC criterion with $\Delta\left(A I C_{i}\right)$ ranging from 1.6 to 2.7. The SBC condition selects only the exponential distribution, which has only one parameter. The thickness dataset spans only half an order of magnitude and is therefore not as well constrained as the spacing dataset which spans three orders of magnitude. Because of the limited scale range, a conservative interpretation with the SBC is preferred to the results suggested by the AIC.

An example of the distributions fitted over a truncated range and extrapolated beyond the truncation bounds is plotted as a histogram of fracture thickness in Figure 


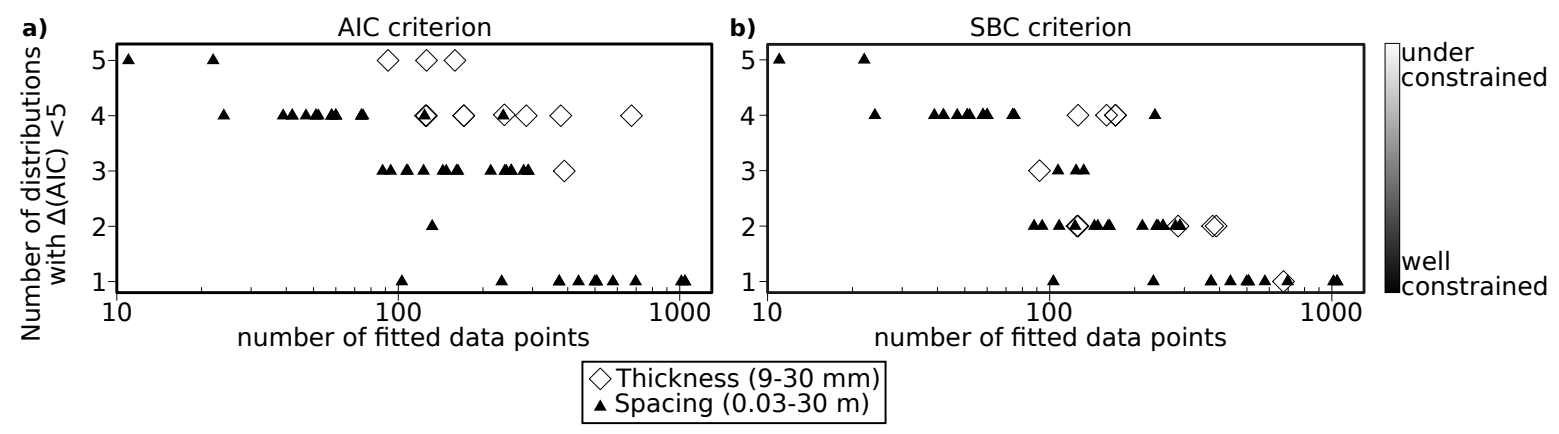

Figure 3.7: Number of distributions satisfying the AIC and SBC conditions as a function of the input number of data points, for the fracture thickness (fitted between 9-30 $\mathrm{mm}$ ) and the fracture spacing (fitted between $0.03-30 \mathrm{~m}$ ). a) AIC condition. b) SBC condition.

3.6c. All distributions fitted with GAMLSS exhibit a good visual fit to the histogram between the truncation bounds, but have different trends outside. The power-law distributions estimated by MLE (via pareto and from MLE estimation of the powerlaw coefficient) have a good visual fit to the histogram, but the power-law with the exponent estimated by least-square on log-log plot does not.

\subsubsection{How Many Fractures Are Enough?}

The number of distributions satisfying the $\mathrm{AIC}$ and $\mathrm{SBC}$ condition $(\triangle(A I C)$ or $\Delta(A I C)$ $<5$ ) depends on both the scale range (interval within truncation limits) and size (number of input data points) of the input dataset. To quantify this dependency, the statistical analysis is made on a series of subsets of different sizes (for different fracture sets) and scale ranges of attribute values (thickness and spacing), resulting in a varying number distributions with $\Delta(A I C)<5$ (Figure 3.7a) or $\Delta(S B C)<5$ (Figure 3.7b). The number of such distributions decreases with increasing number of data points. For the spacing analysis evaluated over three orders of magnitudes $(0.03-30 \mathrm{~m})$, models with fewer than 80 fractures have either four or five models satisfying $\Delta(A I C)<5$ and are under-constrained, whereas datasets with $>300$ fractures are better constrained with only one distribution with $\Delta(A I C)<5$ (Figure 3.7 a). The number of distributions satisfying the AIC condition varies from one to four for 80 to 300 fractures, which relates to how well the measurements follow a theoretical probability distribution.

The spacing dataset spanning a large scale range (three orders of magnitude) has fewer models satisfying the AIC and SBC condition than the thickness dataset which spans only half on order of magnitude. The AIC condition is satisfied for three or four distributions even for thickness datasets containing more than 300 fractures. The SBC condition is satisfied by fewer distributions than the AIC because the SBC further penalises distributions with several parameters.

Moreover, the location of the range of observations considered to be fully sampled affects the number of distributions satisfying the AIC or SBC condition. Tests were 
a) Log-normal best distribution $(\Delta(\mathrm{AIC})=0)$
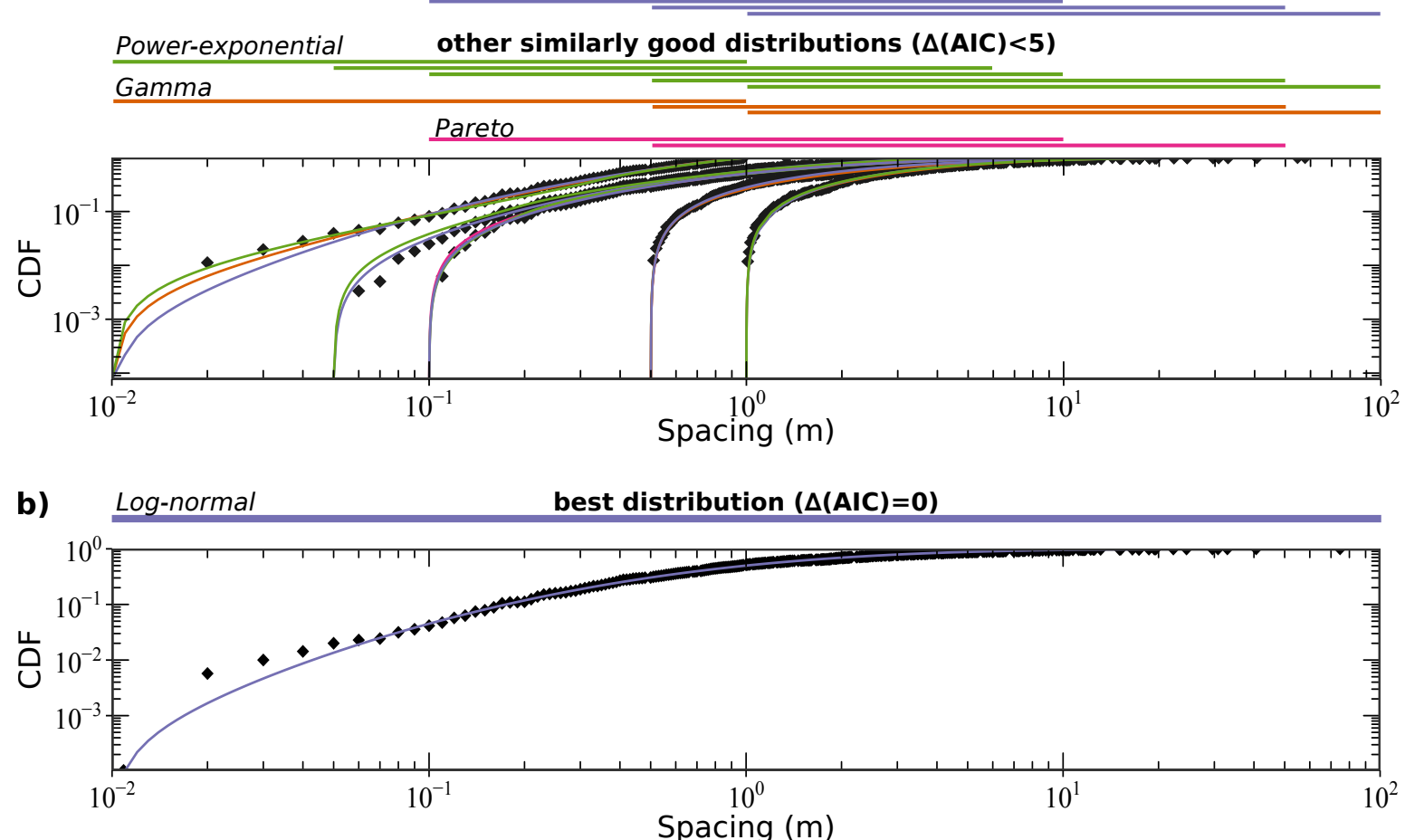

Figure 3.8: Effects of the location of truncating limits on the number and form of distributions satisfying the AIC condition $(\Delta(\mathrm{AIC})<5)$ for the fracture spacing of fracture set 1 in all boreholes. a) CDF of fracture spacing for different truncation intervals (scale range) each covering two orders of magnitude. b) CDF of fracture spacing for the whole range of measured values. The distributions with $\Delta($ AIC $)<5$ are displayed on each corresponding CDF of the data, and as bars at the top of each plot.

performed on a series of fracture spacing datasets for a single fracture set spanning two orders of magnitude, with different truncation limits $(0.01-1 \mathrm{~m} ; 0.05-5 \mathrm{~m} ; 0.1-$ $10 \mathrm{~m} ; 0.5-50 \mathrm{~m} ; 1-100 \mathrm{~m})$. While the best-fitting distribution amongst the five tested is always log-normal, other distributions (power-exponential, gamma and pareto) satisfy the AIC condition for some of the subsets (Figure 3.8 a). In addition, although the data is truncated in reality, we fit a distribution to the whole dataset without limitations on the model or the data. In this case, only the log-normal distribution satisfies the AIC condition (Figure 3.8b), which reflects the higher number of input data. In addition, the log-normal distribution approximates well the under-sampled lower tail of the measurements. Defining the truncation limits of a dataset prior to fitting is thus particularly important, as a log-normal (or gamma) distribution may reflect not simply the distribution of the geological data, but also sampling effects and truncations. 


\subsection{Discussion}

\subsubsection{Sampling Effects Considerations for the Statistical Analysis of Fractures Interpreted in BHTV Logs}

BHTV logs are similar to a scanline sampling in that they measure the orientation and morphological characteristics of fractures intersecting a line, and standard analysis methods are applicable (e.g., Priest, 1993). However, a number of additional precautions must be taken.

The BHTV log quality is affected by a number of logging and drilling artefacts, which can affect the statistical analysis of fracture attributes. In the Rotokawa reservoir, the log quality is affected by the type of logging tool used in these high-temperature boreholes (ABI-85), which had a lower resolution $(5 \times 5 \mathrm{~mm})$ than other standard tools at the times the logs were acquired. In addition, some signal was commonly lost in parts of the borehole due to spalling from the borehole wall on the high side of the deviated boreholes, and in possible intensely fractured areas. Finally, the andesitic rocks imaged by the BHTV logs in the Rotokawa reservoir are likely to have a high roughness at the borehole wall, which decreases the amplitude of the returned signal and thus the ability to detect signal related to fractures within the noise. As the statistical analysis of fracture thickness and spacing is highly dependent on the scale range of observations, where the data is fully observed (within truncation limits), it is important to evaluate image quality effects in each BHTV log.

Some types of fractures may exhibit a similar acoustic amplitude response to that of the host formation, depending on their mineralisation, and are thus difficult to detect on BHTV logs. While such detection issues were not deemed to be serious in the Rotokawa BHTV logs, they could have an effect on the fracture detection and subsequent statistical analysis in other situations.

The Terzaghi correction for orientation sampling bias improves the delineation of fracture sets, but does not rectify the lack of fractures sub-parallel to the borehole $\left(<10^{\circ}\right.$; Figure 3.3). As with measurements of fractures along scanlines of different orientations spanning the same outcrop, a combination of boreholes drilled in different directions allows the sampling of all fracture set directions. For example, the clustering analysis conducted here highlighted the possible presence of a fourth fracture set that had not been identified previously. Fracture set "4", which appears to be linked to an orientation sampling bias in boreholes RK18L2 and RK32, forms a separate cluster in RK30L1 in a direction not affected by the orientation bias (Figures 3.3, 3.A.1). The orientation of this fracture set is consistent with the presence of normal faults of Andersonian orientations (dipping $60^{\circ}$ and striking parallel to $\mathrm{S}_{\mathrm{Hmax}}$ ), which are to be expected in the Rotokawa geological setting. However, the assumption that 
fracture orientations are similar in each borehole is not always applicable, due for example to the presence of active faults that may locally change the in-situ stress and the resulting fracture orientations (McNamara et al., 2015), or to different structural compartments within which fracture orientations vary laterally or with depth. For example, fracture set " 3 " is observed in one borehole (RK30L1) but very rarely in the two others available in this study, which suggests that fractures of this orientation are not present homogeneously throughout the reservoir.

\subsubsection{Uncertainties, and Multiple "Best" Solutions}

The clustering of fracture orientation and the statistical analyses of fracture thickness and spacing both reveal that several "best-fits" often occur. There are at least two good clustering configurations of fracture orientations for each borehole and the combined dataset (Appendix 3.A). Comparisons between several fracture set delineation techniques (Fisher contours, K-means, fuzzy K-means and agglomerative clustering) highlight different possible solutions for a single dataset. The agreement between different techniques, in the context of relevant geological setting, provides the best delineation of fracture sets.

The maximum likelihood estimation method, combined with the AIC and SBC, is able to identify the distribution best approximating a dataset amongst a given set of distributions, as well as other distributions that also have similarly good fits to the data. The limited range of observations for fracture thickness limits the ability to identify a single best-fit distribution. Even for the spacing analysis which has a wider range of observations, a minimum of 100 data points is necessary to identify a single distribution fitting the dataset significantly better than all other tested distributions. In the Rotokawa reservoir, 1218 fractures were identified in the BHTV logs, but the sampling biases and variable BHTV log quality reduce the number of fractures usable for statistical analysis. In addition, the requirements of studying each fracture set individually divides the dataset into subsets, sometimes too small to identify the type of distribution they follow.

The ranking of the likelihood of a distribution using the AIC and SBC is relative, and does not guarantee that the data follows one of the models, so it is important to test different distributions relevant to the type of studied data. Goodness-of-fit tests such as Chi-squared or Kolmogorov-Smirnov tests may be used (James et al., 2013), particularly if fitting only one or two distributions. In this study, however, the objective of the statistical analysis is to evaluate which of the probability distributions commonly found in geological settings best fit the data for further geological interpretation and fracture modelling. As such, a relative ranking of these most common distributions encountered in geological settings is sufficient. However, we have also ensured that the distributions with $\Delta(A I C)<5$ have good fits to the data (i.e. absolute rather than 

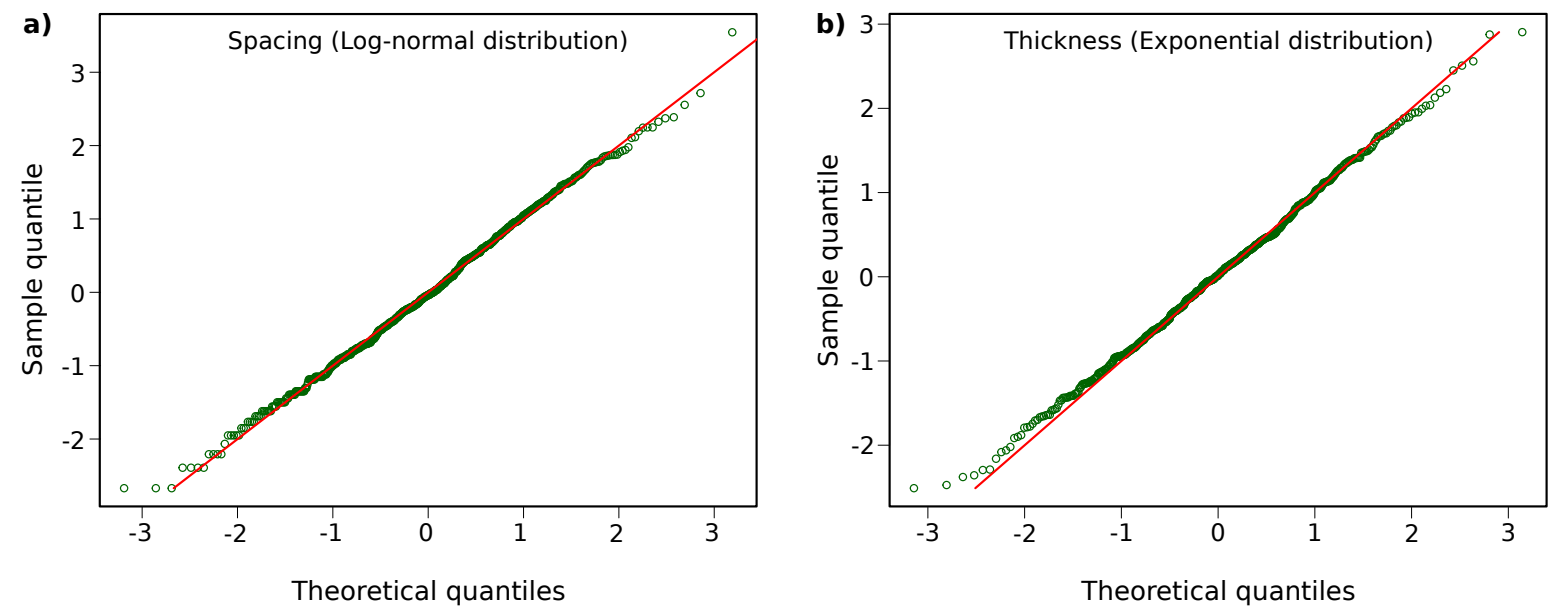

Figure 3.9: Examples of Q-Q plots of fitted distributions; a good alignment between the sample and theoretical quantiles on the line $y=x$ (red line) indicates a good fit. a) Log-normal distribution of spacing in fracture set 1 in all wells (Figure 3.6a). b) Exponential distribution of thickness in all wells (Figure $3.6 \mathrm{~b}$ ).

relative best fit) by inspecting Q-Q plots (Figure 3.9p. When several distributions are satisfying the AIC and SBC conditions, additional datasets covering different scales are required to decide which distribution best approximates the attribute (McCaffrey et al., 2003; Torabi and Berg, 2011). For example, combining BHTV log thickness data with measurements of millimetre-thick (or smaller) fractures in drill-cores would help constrain which of the exponential, log-normal, or even power-law distributions best represent the fracture thickness population over its entire range.

Given the uncertainties associated with the statistical analysis of fracture thickness and spacing, it is desirable to consider several scenarios which each fit the data in order to describe the fracture system, rather than a single configuration.

\subsubsection{Multivariate Analysis}

Maximum likelihood estimation of the distribution of fracture attributes using truncated distributions, presented here for thickness and spacing, is applicable to other fracture attributes such as length. In this work, the analyses of orientation, thickness and spacing are made independently. However, the delineation of fracture sets using a combination of fracture attributes measured on scanlines (e.g., orientation, length, spacing and roughness) can better characterise the fracture system than when using orientation alone as shown by, for example, Tokhmechi et al. (2011) and Hofrichter and Winkler (2006) in engineering and fractured reservoirs applications, respectively. Such multivariate classifications may be applicable to BHTV log measurements in hightemperature geothermal reservoirs, as correlations were found between permeability and cross-cutting fractures interpreted from BHTV logs at the Kawerau geothermal field, Taupo Volcanic Zone (Wallis et al., 2012a). Classifications of fracture attributes measured on scanlines have previously been made using K-means Tokhmechi et al., 
2011) and fuzzy K-means clusterings (Hammah and Curran, 1998), neural networks (Sirat and Talbot, 2001) and Parzen classifiers (Tokhmechi et al., 2011), the latter two using training and testing subsets of the whole datasets. BHTV logs do not provide as detailed information of fracture attributes as scanlines (e.g., length, roughness, infilling). However, other types of wireline logs can be jointly interpreted with fracture dataset interpreted in BHTV logs, using principal component analysis to identify lithologies (Townend et al., 2013) or fuzzy logic to identify permeable zones in oil boreholes (Masoudi et al., 2012). In these cases, the distance measure ("dissimilarity") has to be compatible between orientation data, continuous data (e.g., thickness; Hammah and Curran, 1999), categorical data (e.g., cross-cutting relationships), and other wireline log data sampled at different resolutions.

\subsection{Conclusion}

BHTV logs provide near-continuous records of fracture geometrical characteristics at depth. The application of clustering algorithms extends the fracture orientation analysis made by analysts using stereonets, by providing transparent classifications of fracture orientations into distinct groups, and metrics with which to choose the most appropriate number of clusters. Maximum likelihood estimation (MLE) methods applied to truncated distributions are suitable for evaluating the distribution of fracture attributes (presented here for spacing and thickness) affected by sampling biases. The AIC and SBC are simple tools with which to select distributions best representing the data over the truncated range, amongst a set of distribution forms. Distributions evaluated within truncation ranges where they are deemed fully sampled can then be extrapolated over the entire range of values of the studied attribute. A suitable level of BHTV log quality also needs to be assessed to limit the effects of sampling bias on fracture attribute analysis. Depending on the number of input fracture data, and the range of values they cover (in both size and location), AIC and SBC may rank more than one distribution as similarly good approximations of the data. This statistical approach to fracture attribute analysis, presented here for orientation, thickness and spacing, provides a series of realistic approximations which can be used to constrain reservoir-scale fracture models.

\section{Acknowledgments}

We are grateful to the Rotokawa Joint Venture (Mercury New Zealand and Tauhara North No.2 Trust) for allowing publication of this work. 


\section{Appendix}

\section{A Evaluation of the Number of Orientation Clus- ters for Each Borehole}

Figure 3.A.1 presents the metrics used to choose the best clustering of fracture orientation interpreted in the three boreholes, and the combined dataset comprising data from all three boreholes.
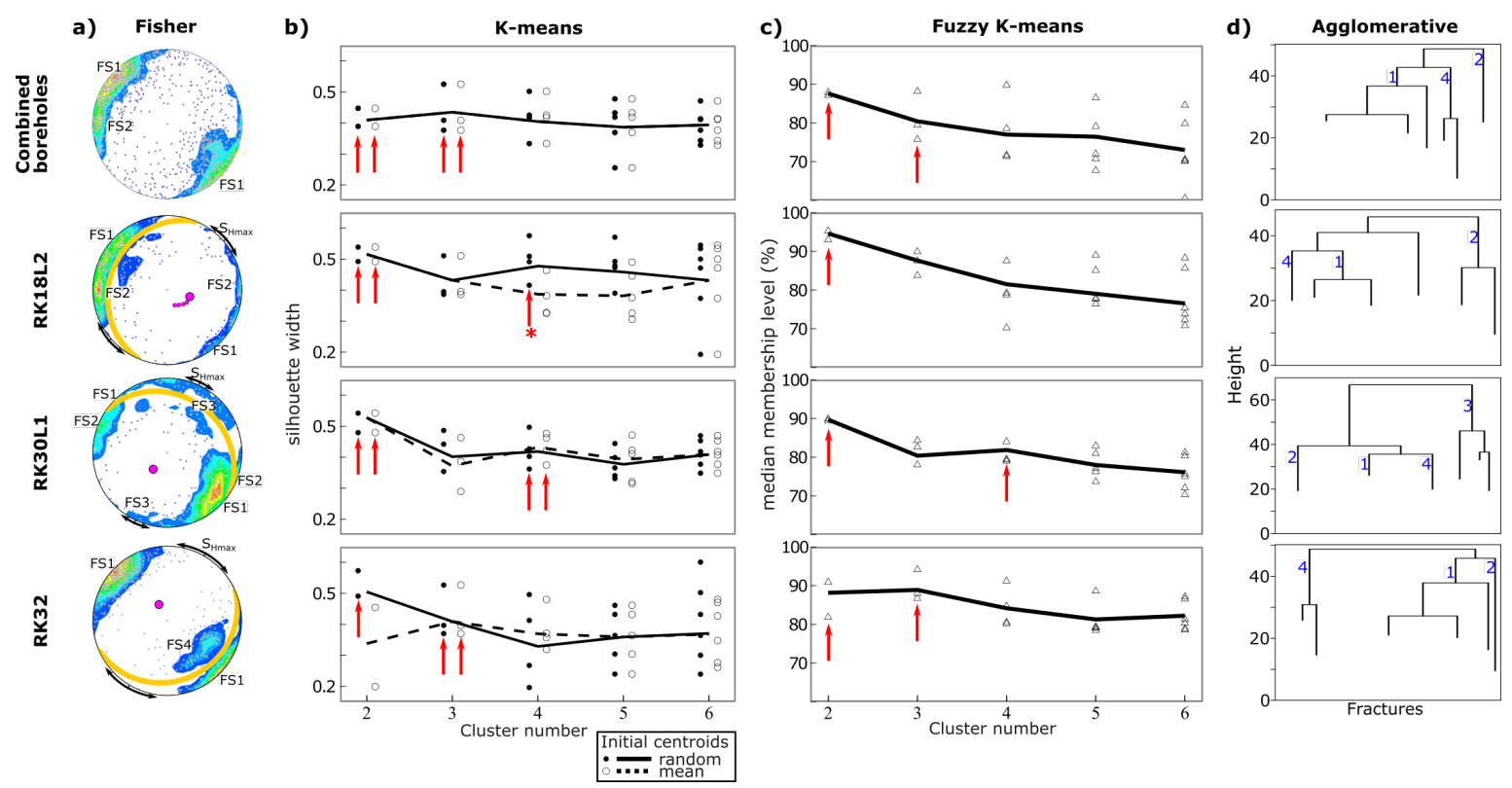

Figure 3.A.1: Evaluation of the optimal number of cluster for each of the three boreholes and combined. a) Fisher contouring (percentages) of poles to fracture planes with resulting fracture sets (FS), $\mathrm{S}_{\text {Hmax }}$ orientation (mean value and one standard deviation; McNamara et al. 2015), and great circle with the orientation of poles to planes of fractures not sampled because they are parallel to the borehole (the pole of this great circle is the borehole trajectory). Stereonets are displayed as lower hemisphere Schmidt projection. (b) K-means: average silhouette width for each cluster (dots), and average of the average silhouette widths (line) for each number of clusters; clusterings are made with random initial centroids, and initial centroids located at the mean orientation of the fracture set. c) Fuzzy K-means: median membership level for each cluster (triangle), and median membership level (line) for each number of clusters. d) Dendogram representing the agglomerative clustering, with the level at which each of the four main fracture sets emerge. The best clusterings are indicated by the underlying arrows in b-c. 


\section{B Probability Distribution Formulas}

Table 3.B.1: Probability density function formulas of distributions used in Chapters 3 and 4 (GAMLSS formulations noted ${ }^{*}$ ), and conversions from GAMLSS formulations to other common formulations.

\begin{tabular}{lll}
\hline Distribution & Probability density function formulations & Conversion \\
\hline \multirow{2}{*}{ Exponential } & $f_{y}(y \mid \mu)^{*}=\frac{1}{\mu} \exp \left(-\frac{1}{\mu} y\right)$ & $\lambda=\frac{1}{\mu}$ \\
& $f_{y}(y \mid \lambda)=\lambda \exp (-\lambda y)$ & \\
\hline Gamma & $f_{y}(y \mid \mu, \sigma)^{*}=\frac{y^{\left(\frac{1}{\sigma^{2}}-1\right)} \exp \left[-\frac{y}{\sigma^{2} \mu}\right]}{\left(\sigma^{2} \mu\right)^{\frac{1}{\sigma^{2}}} \Gamma\left(\frac{1}{\sigma^{2}}\right)}$ & $a=\frac{1}{\sigma^{2}} ;$ \\
& $f_{y}(y \mid a, s)=\frac{1}{s^{a} \Gamma(a)} y^{(a-1)} \exp [-(y / s)]$ & $s=\sigma^{2} \mu$ \\
\hline
\end{tabular}

Log-normal $\quad f_{y}(y \mid \mu, \sigma)=\frac{1}{y \sqrt{2 \pi} \sigma} \exp \left[-\frac{1}{2}\left(\frac{\log y-\mu}{\sigma}\right)^{2}\right]$

Pareto*

$f_{y}(y \mid \mu, \sigma)^{*}=\frac{1}{\sigma} \mu^{\frac{1}{\sigma}}(y+\mu)^{-\left(\frac{1}{\sigma}+1\right)}$

$\alpha=\left(\frac{1}{\sigma}+1\right)$

for $\mu \ll y$

Power-law $\quad f_{y}(y \mid \alpha)=C * y^{-\alpha}$ for $\alpha>1$

$\alpha$ estimated by least-square regressions in log-log

plots, and by MLE following Clauset et al. (2009):

$\alpha=1+n\left[\sum_{i=1}^{n} \frac{y_{i}}{y_{\text {min }}}\right]^{-1}, y_{i}>y_{\min }$

Power-

$f_{y}(y \mid \mu, \sigma, \nu)^{*}=\frac{\frac{1}{\sigma}\left(\nu \exp \left[-0.5|z / c|^{\nu}\right]\right)}{c \cdot 2^{1+\frac{1}{\nu}} \cdot \Gamma\left(\frac{1}{\nu}\right)}$ where

exponential

$$
c=\left[\frac{2^{-\frac{2}{\nu}} \Gamma\left(\frac{1}{\nu}\right)}{\Gamma\left(\frac{3}{\nu}\right)}\right]^{0.5}
$$

$f_{y}\left(y \mid \beta, \lambda, y_{\text {min }}\right)=\beta \lambda \exp \left[\lambda y_{\text {min }}^{\beta}\right] y^{\beta-1} \exp \left[-\lambda y^{\beta}\right]$

The GAMLSS log-normal distribution formulation is the common formulation. All variable $(y)$ and parameters $(\mu, \sigma$ and $\nu)$ are strictly positive, except for $\mu$ of the log-normal and power-exponential distributions. 


\section{Tectonic, Lithologic and Thermal Controls on Fracture System Geometries in an Andesitic High-Temperature Geothermal Field}

\subsection{Abstract}

Analysis of fracture orientation, spacing and thickness from acoustic borehole televiewer (BHTV) logs and cores in the andesite-hosted Rotokawa geothermal reservoir (New Zealand), constrains potential controls on permeability. Cluster analysis of fracture orientations identifies four fracture sets. Probability distributions of fracture spacing and thickness measured on BHTV logs are estimated using maximum likelihood estimations applied to truncated size distributions to account for sampling bias. Fracture spacing is dominantly log-normal, though two subordinate fracture sets have powerlaw spacing. This difference in spacing distributions may reflect the influence of the andesitic sequence stratification (log-normal) and tectonic faults (power-law). Fracture thicknesses of 9-30 mm observed in BHTV logs, and 1-3 mm in cores, are interpreted to follow a power-law distribution. Fractures in thin-sections $(\sim 5 \mu \mathrm{m}$-thick) do not fit this power-law distribution, which indicate a change of controls on fracture thickness from internal (such as thermal) controls at thin-section scale to external (lithological or tectonic) at core and BHTV scales of observation. However, the $\sim 5 \%$ volumetric percentage of fractures within the rock at all three scales suggests a self-similar behaviour in 3-D. Power-law thickness distributions potentially associated with power-law fluid flow rates, and increased connectivity where fracture sets intersect, may contribute to the large permeability variations that occur at hundred-meter scales in the reservoir. The described fracture geometries can be incorporated into fracture and flow models to explore the roles of stress, connectivity, and mineral precipitation/dissolution on permeability in such geothermal systems. 


\subsection{Introduction}

Non-sedimentary rocks are increasingly recognised as fluid reservoirs. Volcanic formations host geothermal Arnórsson, 1995, Nemčok et al., 2004, Wilson and Rowland, 2016), groundwater Lachassagne et al., 2014), hydrocarbon (Feng, 2008), and mineral Spörli and Cargill, 2011) resources, while also being capable of storing unwanted $\mathrm{CO}_{2}$ (Pollyea et al., 2014; Matter et al., 2016) and radioactive waste (Chen et al., 2001), and capable of supporting surface engineering projects Alemdag, 2015). The geometry of vesicles and fractures in volcanic units has been extensively studied in outcrops (e.g., DeGraff and Aydin, 1987; Gudmundsson, 2000; Conway et al., 2015) but can be difficult to quantify in reservoirs due to sparse cores and wireline logging measurements. In lava-hosted geothermal reservoirs, permeability is strongly influenced by faults and fractures (e.g., Nemčok et al., 2007; Davatzes and Hickman, 2010; McNamara et al., 2015), but is often not sufficiently well constrained to make accurate fluid flow predictions (Aprilina et al., 2015; Hernandez et al., 2015; Kissling et al., 2015b).

Description of the three-dimensional (3-D) geometry of a fracture system is necessary to develop reservoir-scale models which can assist resource management Bonnet et al., 2001; Berkowitz, 2002; Chilès, 2005), and constrain the origin and evolution of the fracture systems (Ackermann et al., 2001; McCaffrey et al., 2003). Fracture systems can be described in terms of the orientation of different fracture sets, and the probability distribution of fracture attributes: aperture (for open fractures), thickness (for veins), spacing, density and length (Priest, 1993). The probability distribution of fracture attributes varies as a function of the processes that control fracture generation and propagation (Bonnet et al., 2001; André-Mayer and Sausse, 2007). In layered systems, joint spacing is usually best approximated by regular, log-normal, gamma, or exponential distributions, with characteristic sizes linked to the layer thickness and ratio of the layer tensile to interface shear strength Gillespie et al. 1993 ; Rives et al., 1992 Schöpfer et al., 2011). Fluids tend to flow along these joints and contacts between layers (Figure 4.13). In contrast, the spacing of fractures close to faults are commonly clustered according to power-law distributions which are scale-independent (Figure 4.1b, see Bonnet et al. (2001) for review; McCaffrey et al. (2003); Johri et al. (2014)).

In reservoirs, the scaling of fracture attributes is evaluated from typically sparse cores and borehole image logs such as acoustic borehole televiewer (BHTV) logs, which provide an oriented image of the inside of a borehole (Hansen and Buczak, 2010). In this study, we analyse fracture orientation, thickness, spacing, and volumetric densities at BHTV log and core scales, complemented by previously reported measurements on thin-sections, from the Rotokawa Geothermal Field. This high-temperature, faulted geothermal reservoir is located in the Taupo Volcanic Zone (New Zealand), 
a)

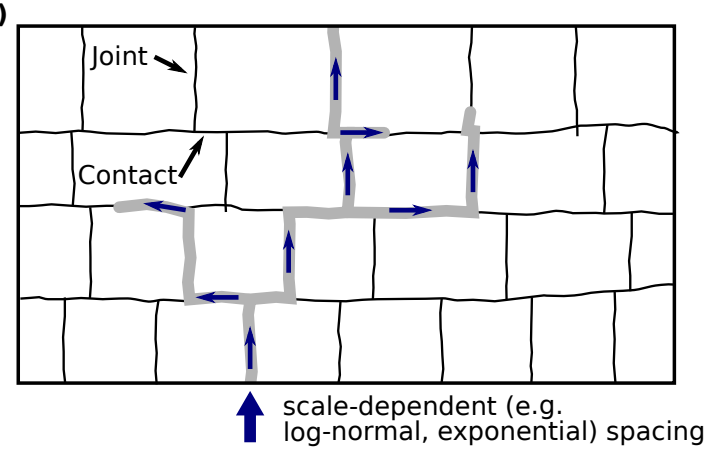

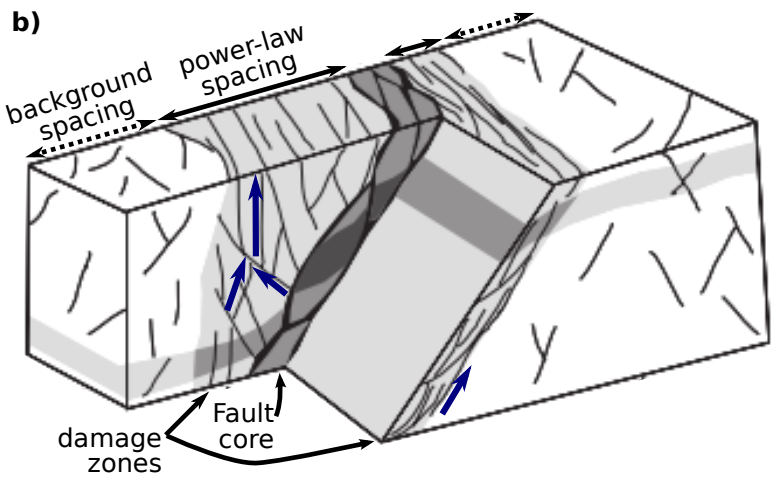

Figure 4.1: Schematic fracture and fluid flow models. a) In layered jointed rocks, flow can occur along joints and contacts between layers; the layer thickness and ratio of layer tensile to interface shear strength (Schöpfer et al. 2011$)$ are characteristic scales that affect the joint spacing (after Gudmundsson et al., 2002). b) In fault damage zones, fracture spacing is clustered near faults (commonly power-law), and a background spacing (power-law or scale-dependent) prevails away from the fault zone (after Manzocchi et al., 2010).

and hosted in andesitic lavas and breccias (McNamara et al., 2016a, and references therein). Within the reservoir, fractures are thought to be the major control on the flow of hot fluids (McNamara et al., 2015). However, little has been conducted to date on the fracture geometries and densities, or how they control permeability. Here, we infer mechanisms controlling fracture distributions in the reservoir from statistical analysis of fracture attributes, with general applications to constrain geometric fracture networks in andesite-hosted geothermal reservoirs.

\subsection{Geological Settings}

The Rotokawa Geothermal Field is located $\sim 10 \mathrm{~km}$ north of Taupo, New Zealand, on the eastern side of the Taupo Volcanic Zone (TVZ; Figure 4.2). The TVZ is the active, southern portion of the Lau-Havre-Taupo extensional intra-arc basin, that formed as a result of the oblique subduction of the Pacific plate beneath the Australian plate $B e g g$ and Mouslopoulou, 2010; Rowland and Simmons, 2012; Wilson and Rowland, 2016).

The TVZ is $\sim 350 \mathrm{~km}$ long and up to $\sim 60 \mathrm{~km}$ wide, extending from the Ruapehu volcano to the Bay of Plenty and beyond to White Island (Wilson et al., 1995$)$. Clockwise vertical-axis rotation of the eastern North Island has accompanied the formation of NE-SW-striking normal faults defining the Taupo Rift, resulting in a contemporary NW-SE-directed extension rate of the order of $1 \mathrm{~cm} /$ year Wallace et al., 2004, Nicol et al. 2006). These normal faults are laterally discontinuous and typically dip 60-80 at the ground surface (Rowland and Sibson, 2001; Villamor and Berryman, 2001, 2006; Rowland et al., 2010; Seebeck et al., 2010).

The Rotokawa Geothermal Field is an operating geothermal field with a total in- 


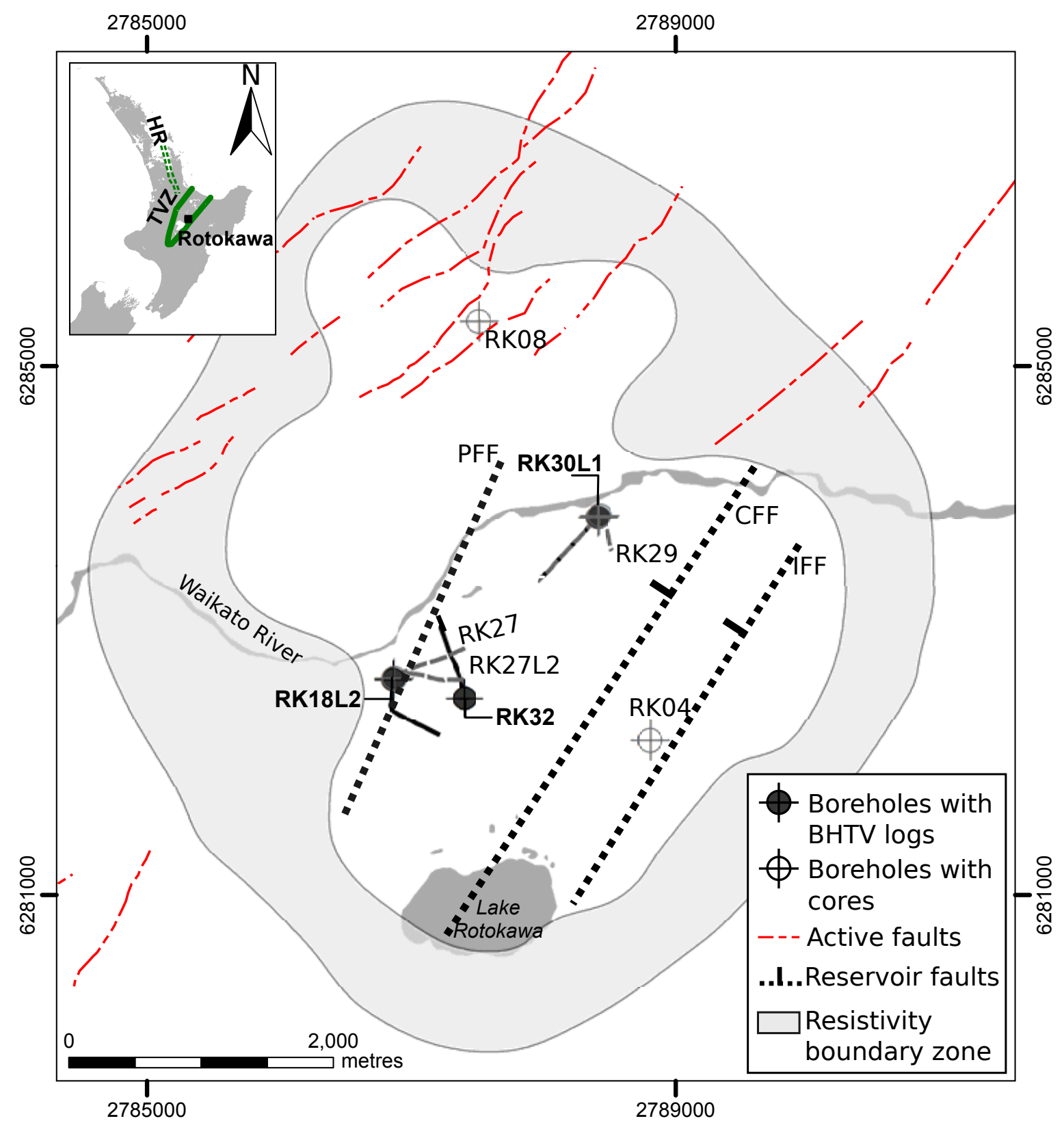

Figure 4.2: Location map of the Rotokawa Geothermal Field including the approximate resistivity boundary zone at about $500 \mathrm{~m}$ depth (after Risk, 2000), the mapped active faults (GNS Science active fault database, http://data.gns.cri.nz/af/; Litchfield et al. (2014)), the inferred faults projected from the top of the Rotokawa Andesite (Wallis et al., 2013), and the location of the boreholes where the cores and three BHTV logs used in this study have been collected. PFF: principal field fault; CFF: central field fault; IFF: injection field fault. Insert displays the location of the Rotokawa Geothermal Field, Taupo Volcanic Zone (TVZ), and the Hauraki Rift (HR). 
stalled electricity production capacity of $174 \mathrm{MW}$ (Hernandez et al., 2015). The geology of the field comprises volcano-sedimentary and andesitic formations $\leq 1.9 \mathrm{Ma}$ Chambefort et al. 2014), which overlie older faulted Rotokawa Andesite units and the Mesozoic Greywacke basement (McNamara et al. 2016a). The Rotokawa Andesite is an 800$2100 \mathrm{~m}$-thick unit of andesite lavas and breccias and is inferred to form a large buried andesitic volcano which is also partly located under the nearby Ngatamariki Geothermal Field (Browne et al., 1992; Chambefort et al., 2014). The deep, hot $\left(320^{\circ} \mathrm{C}\right)$ aquifer is hosted in these low matrix porosity (4-15\%) andesites Siratovich et al., 2014; Hernandez et al., 2015), in which fluid flow is mostly controlled by fractures and faults (McNamara et al., 2015). Three large (0-400 m throw) NE-SW-striking normal faults have been inferred within the geothermal field based on borehole stratigraphy, reservoir temperatures, and microseismicity (Figure 4.2, Wallis et al., 2013; Sherburn et al., 2015). The faults generate offsets observed in the Greywacke basement and Rotokawa andesites, but are thought not to propagate into the overlying units where stratigraphic offsets between wells are not observed. These faults are consistent with a normal faulting regime (Davidson et al., 2012) and an azimuth of maximum horizontal compression stress $\left(\mathrm{S}_{\mathrm{Hmax}}\right)$ oriented approximately NE-SW, although variations in $\mathrm{S}_{\mathrm{Hmax}}$ occur between and within wells (McNamara et al., 2015). The reservoir has a heterogeneous permeability distribution, for example with one of the three main faults inferred to form a barrier to flow (Hernandez et al., 2015; Sherburn et al., 2015).

\subsection{Data}

\subsubsection{Cores}

Cores in the Rotokawa Geothermal Field display a variety in their fracture attributes (density, orientation, infilling minerals), volcanic lithologies, and hydrothermal alteration (Figure 4.3). Volcanic rocks sampled by the cores have been subject to pervasive alteration consisting of a propylitic-style hydrothermal assemblage (quartz, wairakite, epidote, clinozoisite, illite, chlorite, adularia, albite and calcite), with an intensity varying from weak to strong (McNamara et al., 2016a, and references therein). Average clast size of breccias (Figure $4.3 \mathrm{c}$ ) is $26 \pm 22 \mathrm{~mm}$ (one standard deviation). Although most cores comprise only one lithology (Table 4.1), alteration intensity can vary at decimetre scale, and both andesite lava and breccia can occur over tens of centimetres (Figure 4.3 e).

In this study, fractures were systematically described in 58 non-oriented cores from 19 boreholes. Each fracture is described in terms of its location along the core, dimensions (height, length), orientation relative to the core axis, termination (intersection with other fractures or thinning), mineralisation, thickness (between vein walls), and 


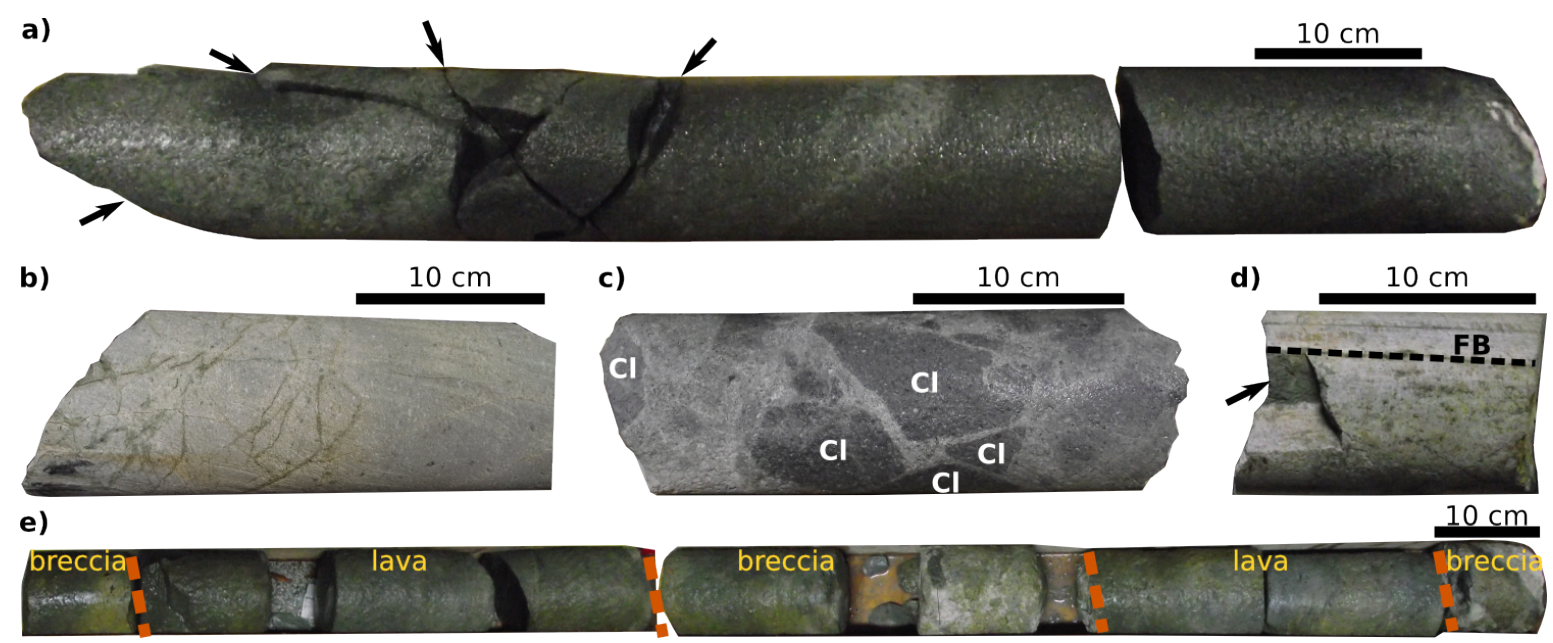

Figure 4.3: Lithologies, alteration and fracturation observed in cores from the Rotokawa Geothermal Field. a) Moderately altered andesite lava with low fracture density (arrows indicate natural fractures); RK27L2 \#1. b) Strongly altered andesite lava with high (on the left) to low (on the right) epidote-filled fracture density; RK29 \#1. c) Moderately altered andesite breccia with large clasts ("Cl") and low fracture density. Thin white calcite and quartz veins surround the clasts; RK27 \#1. d) Flow-banded rhyolite lava with fracture (arrow) aligned with flow-banding ("FB"); RK4 \#3. e) Intercalation of andesite lava and breccia (dashed lines between lithology changes). The core names refer to Table 4.1 .

aperture (open space between fracture walls). Measurements were made on the outside of cylindrical cores, except in the case of RK27L2 where the core was slabbed. Fracture density measured on the slabbed core can be compared to non-slabbed cores. Most cores were fragmented, due to either pre-drilling fractures or to drilling-induced fractures. In addition, numerous cores have degraded since their collection up to 30 years ago. The measure of fracture density is strongly dependent on the state of the core, and quantitative analysis was only performed on core pieces exceeding $10 \mathrm{~cm}$ in length, in cores deemed to be sufficiently preserved. Fractures induced by drilling and handling of the core were identified by their non-planarity and lack of mineralisation, and are not included in the dataset (Kulander et al. 1990$)$. In total, 181 natural fractures were sampled from $14.8 \mathrm{~m}$ of core in 5 boreholes which penetrated andesite lavas, rhyolite lava (Haparangi rhyolite) and breccias (Table 4.1). In addition, six fractures were sampled from two cores of partially-welded to welded tuff of the Wairakei Ignimbrite. Fractures are partially to fully infilled with calcite, epidote, quartz, chlorite, other clays, Fe-oxide, and pyrite (Figure 4.3). Some fractures show slickenslides consistent with shear displacement.

\subsubsection{BHTV Logs}

The location, orientation, thickness, and acoustic amplitude is measured in BHTV logs for each fracture intersecting the borehole which has a acoustic sufficient contrast with 


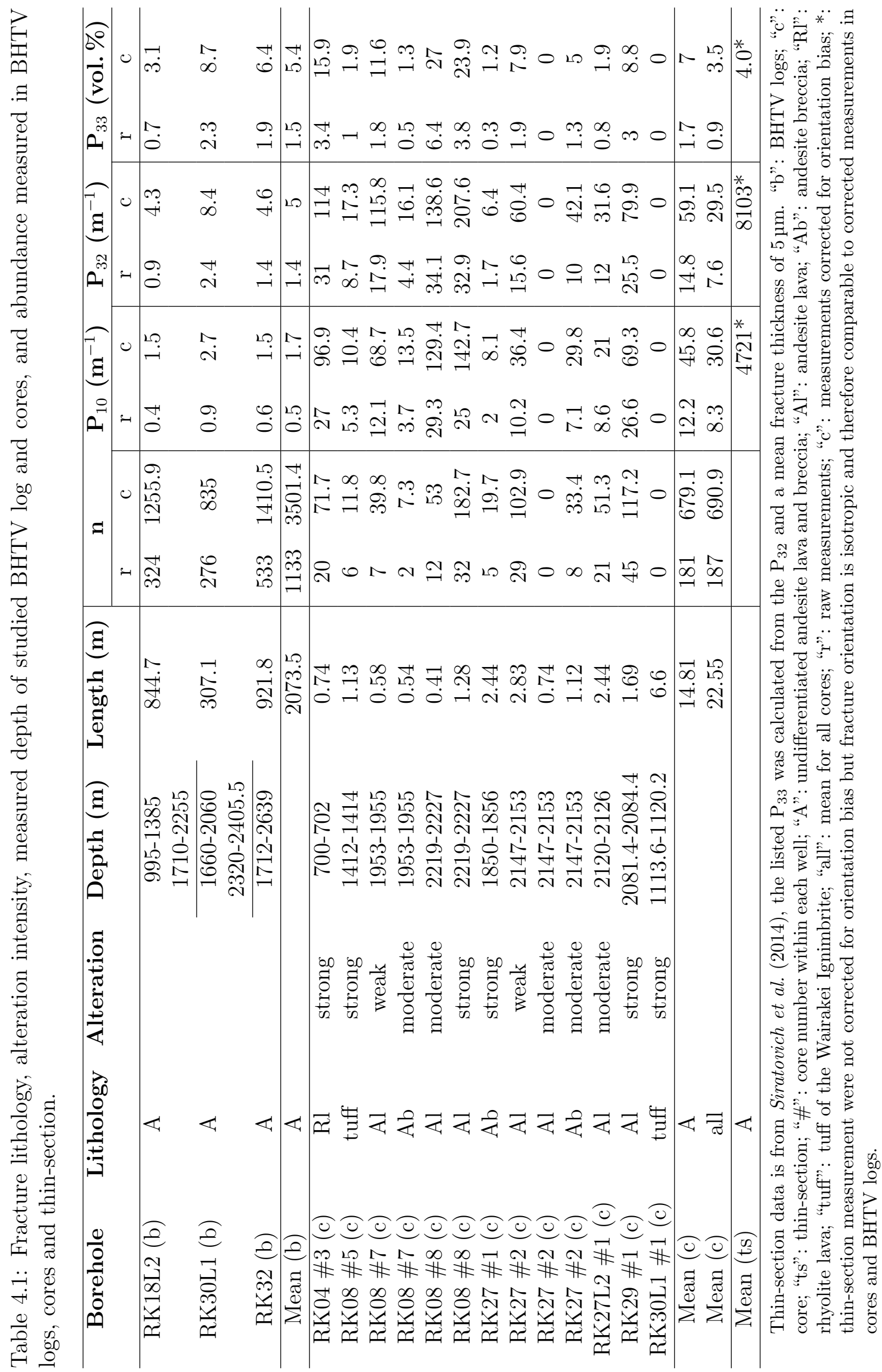



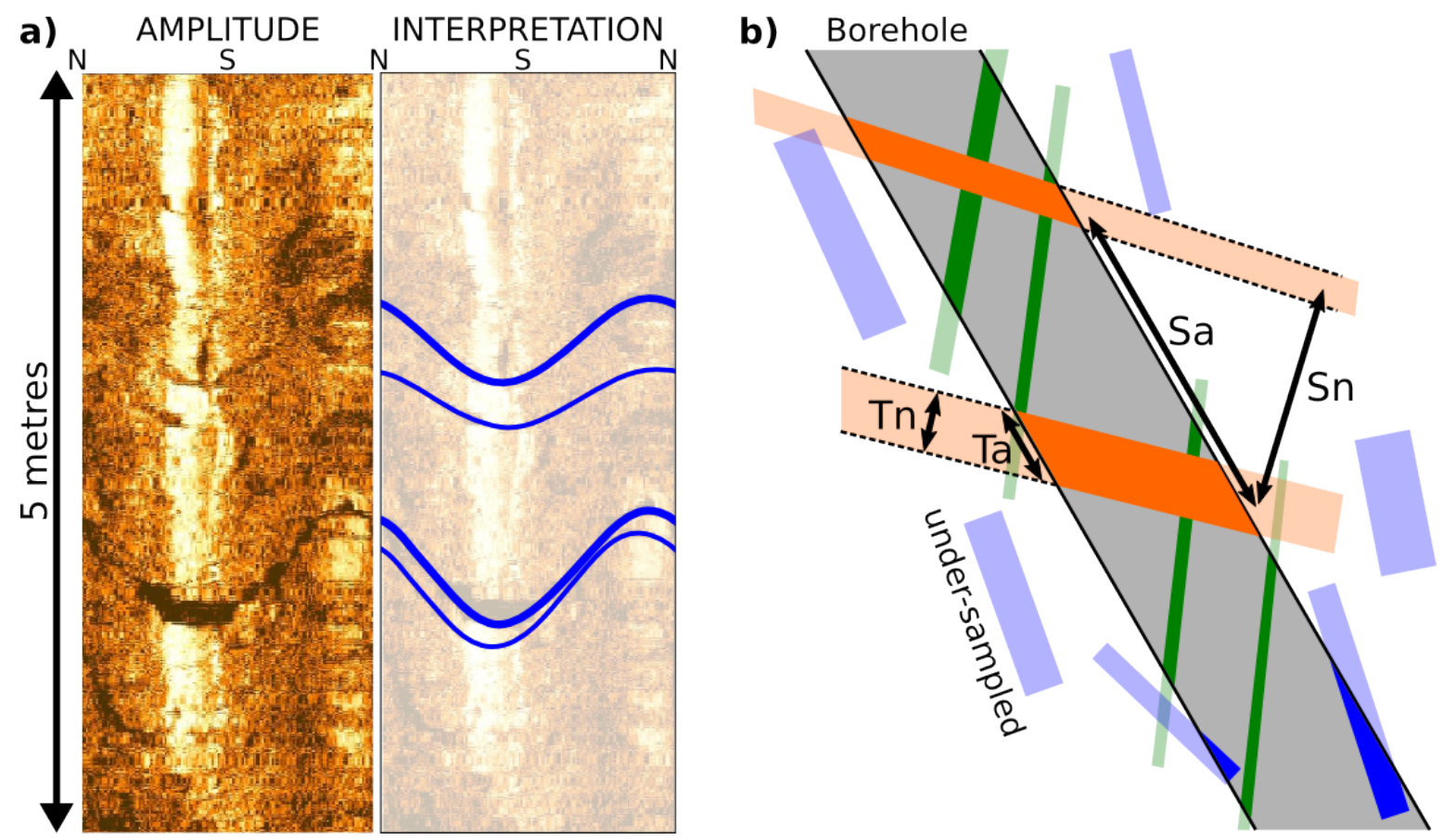

Figure 4.4: Example of a BHTV log and fracture measurements. a) Unwrapped acoustic amplitude image oriented to north, and interpretation of planar fractures appearing as sinusoids. b) Schematic of an inclined borehole with three fracture sets (orange, green and blue), showing measurements of apparent thickness (Ta) and spacing ( $\mathrm{Sa}$ ). Statistical analysis is performed on the fracture-normal thickness ( $\mathrm{Tn}$ ) and spacing (Sn). Fractures parallel to the borehole (blue set) are undersampled.

the background formation (Figure 4.4a; Massiot et al., 2015). BHTV logs alone cannot differentiate between hydraulically conductive and non-conductive fractures, so we use the term "thickness" instead of "aperture" to describe the distance between the two fracture walls. We use the term "fractures" to refer to discontinuities, including open fractures, faults with offsets, and veins.

Three BHTV logs were acquired in the Rotokawa Geothermal Field in boreholes RK18L2, RK30L1 and RK32 (Figure 4.2). The resulting fracture dataset is similar to that presented by McNamara et al. (2015), with more accurate thickness measurements. The fracture analysis presented here focuses on intervals of andesite rocks (lavas and breccias), as defined from cuttings and inferred from the 3-D geological model of the Rotokawa Geothermal Field for intervals where drill-cuttings were not recovered due to total drilling losses (Table 4.1. Wallis et al., 2013). To limit the sampling effects caused by variable BHTV log quality, analysis is only conducted within zones of poor to good quality (i.e. where $\geq 25 \%$ of the circumference of the borehole was observed; Massiot et al., 2015). A total of 1133 fractures were sampled over a cumulative borehole interval of $2074 \mathrm{~m}$ (measured along the length of the deviated boreholes; Table 4.1). 


\subsection{Method}

\subsubsection{Fracture Processing}

Fracture sets of similar orientations are delineated manually using Fisher density contours, and automatically using K-means, fuzzy K-means and agglomerative clusterings (Hammah and Curran, 1998; James et al., 2013, Chapter 3). The distance ("dissimilarity") measure used in the clustering algorithms is the acute angle between each pair of fractures. This dissimilarity measure is not affected by the $180^{\circ}$ difference of dip direction for near-parallel sub-vertical fractures dipping in opposite directions. The best clustering configurations are based on a combination of a high silhouette width for the K-means clustering (Reynolds et al., 2006), a high median membership level for the fuzzy K-means, and visual inspection of agglomerative clustering results (Chapter 3). No fracture-orientation analysis (absolute or relative) was possible in these non-oriented cores, as core sections could only rarely be fitted together.

As is the case with one-dimensional scanline measurements made on outcrops, fractures sub-parallel to the borehole axes and cores are under-sampled (Figure $4.4 \mathrm{~b}$ ). The orientation analysis of the BHTV dataset is made after correction for this orientation bias (Terzaghi, 1965, Massiot et al., 2015). The fracture density of the BHTV and core datasets are reported for both the raw (uncorrected) dataset, and after correction for the orientation bias.

The apparent fracture thickness measured at the borehole wall is converted to the true fracture thickness measured perpendicular to the fracture walls (Figure $4.4 \mathrm{~b} ; B a r$ ton and Zoback, 1992, Massiot et al., 2015). Fracture spacing is measured for each fracture set between adjacent fractures along the borehole. This apparent fracture spacing is converted into the fracture-normal spacing, perpendicular to the mean orientation of the fracture set Priest, 1993). Fracture attributes are generally subject to sampling bias due to their size, with the under-sampling of small values due to the resolution of the dataset, and under-sampling of large values due to their scarcity (Pickering et al., 1995). In the Rotokawa BHTV logs, the fracture thickness population is considered to be fully sampled between 9 and $30 \mathrm{~mm}$, and the fracture spacing between 0.03 and $30 \mathrm{~m}$ (Chapter 3).

\subsubsection{Distribution Fitting}

The probability distribution form and parameters of the fracture thickness and spacing are evaluated following methods described in Chapter 3 . Five probability distributions commonly found in geological systems, namely negative exponential, log-normal, gamma, power-law and power-exponential (Bonnet et al., 2001), are fitted to the fracture thickness or spacing populations within truncation bounds, i.e. where the data 
are inferred to be fully sampled. The fitting is made with maximum likelihood estimations applied to truncated distributions (Stasinopoulos and Rigby, 2007). The power-law distribution is estimated with a pareto distribution. Tests with maximum likelihood estimates of the power-law coefficient (Clauset et al., 2009) provided similar coefficients to the ones derived from the pareto distribution for datasets where the pareto (an hence power-law) distribution was a good fit. Akaike Information Criterion (AIC) and Schwartz Bayesian Criterion (SBC) statistical information criteria rank distributions by how well they approximate the data amongst the five tested distribution Burnham and Anderson, 2002). The difference $\left(\Delta\left(A I C_{k}\right)=A I C_{k}-A I C_{m i n}\right)$ between the lowest $\mathrm{AIC}\left(\mathrm{AIC}_{\text {min }}\right)$ and the $\mathrm{AIC}_{k}$ values of an alternative distribution $k$ decreases as the goodness of fit of the distribution $k$ increases. We considered that models with $\Delta\left(A I C_{k}\right)<5$ (referred to as "the AIC condition") represent the data similarly well (Burnham and Anderson, 2002). The same condition is applied to SBC. Visual inspection of qq-plots ensures that the distributions satisfying the AIC or SBC condition are a good fit to the data in an absolute as well as relative way. AIC and SBC penalise distributions with numerous parameters (such as the power-exponential distribution with three parameters $\hat{\mu}, \hat{\sigma}$ and $\hat{\nu}$ ) compared to those with fewer parameters (gamma, log-normal and pareto distributions have two parameters $\hat{\mu}$ and $\hat{\sigma}$, and the exponential distribution has only one parameter $\hat{\mu}$ ). The penalty term for added parameters is higher for SBC than AIC. We use the AIC for ranking fracture spacing distributions spanning three orders of magnitude, and SBC for fracture thickness distributions spanning only half an order of magnitude, in order to limit over-fitting.

Fracture spacing is determined for each fracture set, and for each orientation clustering configuration (i.e. Fisher density contours, K-means and agglomerative clustering; see Section 4.6.1). Fracture thickness analysis is conducted for each borehole separately and combined, and for each fracture set in the combined dataset. For each case, the distributions satisfying the AIC (or SBC) condition are listed in Tables 4.A.1 and 4.A.2, by decreasing AIC (or SBC) rank. Tables 4.A.1 and 4.A.2 also report the $\hat{\mu}$, $\hat{\sigma}$ and $\hat{\nu}$ parameters of the distribution with the highest AIC rank Stasinopoulos and Rigby, 2007, Chapter 3), and the power-law coefficient derived from the fitted pareto distribution.

\subsubsection{Density Calculations in Cores and BHTV Logs}

Fracture abundance is evaluated here in three ways, corresponding to different dimensionalities of fractures and rock mass (Dershowitz et al. 1999): (1) $\mathrm{P}_{10}$ is the "linear density", defined as the number of fractures $n$ per length of core or BHTV $\log L ;(2)$ $\mathrm{P}_{32}$ is the "areal intensity", the sum of fracture area per volume of rock; and (3) $\mathrm{P}_{33}$ is the "volumetric porosity", the sum of fracture volume per volume of rock. $\mathrm{P}_{33}$, expressed as a percentage, would correspond to the fracture porosity if fractures were 
open. $\mathrm{P}_{32}$ and $\mathrm{P}_{33}$ are often derived from outcrop measurements of the $\mathrm{P}_{21}$ fracture intensity (sum of fracture length per outcrop area) and stereological calculations to extrapolate from 2-D measurements to 3-D parameter (Wang, 2005). Instead, the $\mathrm{P}_{32}$ and $\mathrm{P}_{33}$ values calculated in this work incorporate the weighting coefficients of each fracture used to mitigate the orientation sampling bias correction. Thus, the reported $\mathrm{P}_{32}$ and $\mathrm{P}_{33}$ values corrected for orientation bias are close to being a 3-D parameter.

$\mathrm{P}_{32}$ and $\mathrm{P}_{33}$ parameters are calculated for both the core and BHTV dataset, providing two scales of observation. Fractures are assumed to be planar, and their intersection with the cylindrical cores and boreholes form ellipses. We calculate $\mathrm{P}_{32}$ and $\mathrm{P}_{33}$ as if fractures were confined to the borehole. The areal intensity $\mathrm{P}_{32}$ is expressed as:

$$
P_{32}=\frac{\sum_{i=1}^{n} \pi r a_{i}}{\pi r^{2} L}=\frac{\sum_{i=1}^{n} a_{i}}{r L}
$$

where $a_{i}$ is length of the semi-major axis of the fracture $i$, and $r$ the core or borehole radius, i.e. the length of the semi-minor axis of the ellipse representing the fracture. For the cores, the length of the major axis is measured along the fracture. The BHTV analysis automatically provides the length of the major axis.

$\mathrm{P}_{33}$, the volume of fracture per volume of rock is:

$$
P_{33}=\frac{\sum_{i=1}^{n} \pi r a_{i} t_{i}}{\pi r^{2} L}=\frac{\sum_{i=1}^{n} a_{i} t_{i}}{r L}
$$

where $t_{i}$ is the thickness of fracture $i$. In the following, we describe $\mathrm{P}_{10}$ and $\mathrm{P}_{33} ; \mathrm{P}_{32}$ values presented in Table 4.1 can be used for further fracture modelling.

\subsection{Results}

\subsubsection{Orientation}

The orientations of fractures identified in the available BHTV logs can be separated into four fracture sets (FS) using Fisher contours (Figure 4.5). FS1, FS2 and FS3 are steeply dipping (mean dip magnitudes of $70-90^{\circ}, 80-86^{\circ}$, and $77^{\circ}$ respectively), and strike NESW, N-S and NW-SE, respectively (Tables 4.A.1 and 4.A.2. FS4 strikes NE-SW like FS1, but with a moderate dip $\left(70^{\circ}\right.$ average). FS1 is the dominant orientation, with a strike parallel to the maximum horizontal stress direction $\left(\mathrm{S}_{\mathrm{Hmax}} ;\right.$ McNamara et al. 2015), and is observed in all three boreholes. FS2 is observed in boreholes RK18L2 and RK30L1; FS3 only in borehole RK30L1; and FS4 only in borehole RK32. In the combined dataset which includes all three boreholes, only FS1 and FS2 are defined, as they have much higher Fisher densities than FS3 and FS4. As discussed by McNamara et al. (2015), borehole RK18L2 has more fractures dipping towards the NW than the other two boreholes, but the strike orientations are similar between boreholes. The 


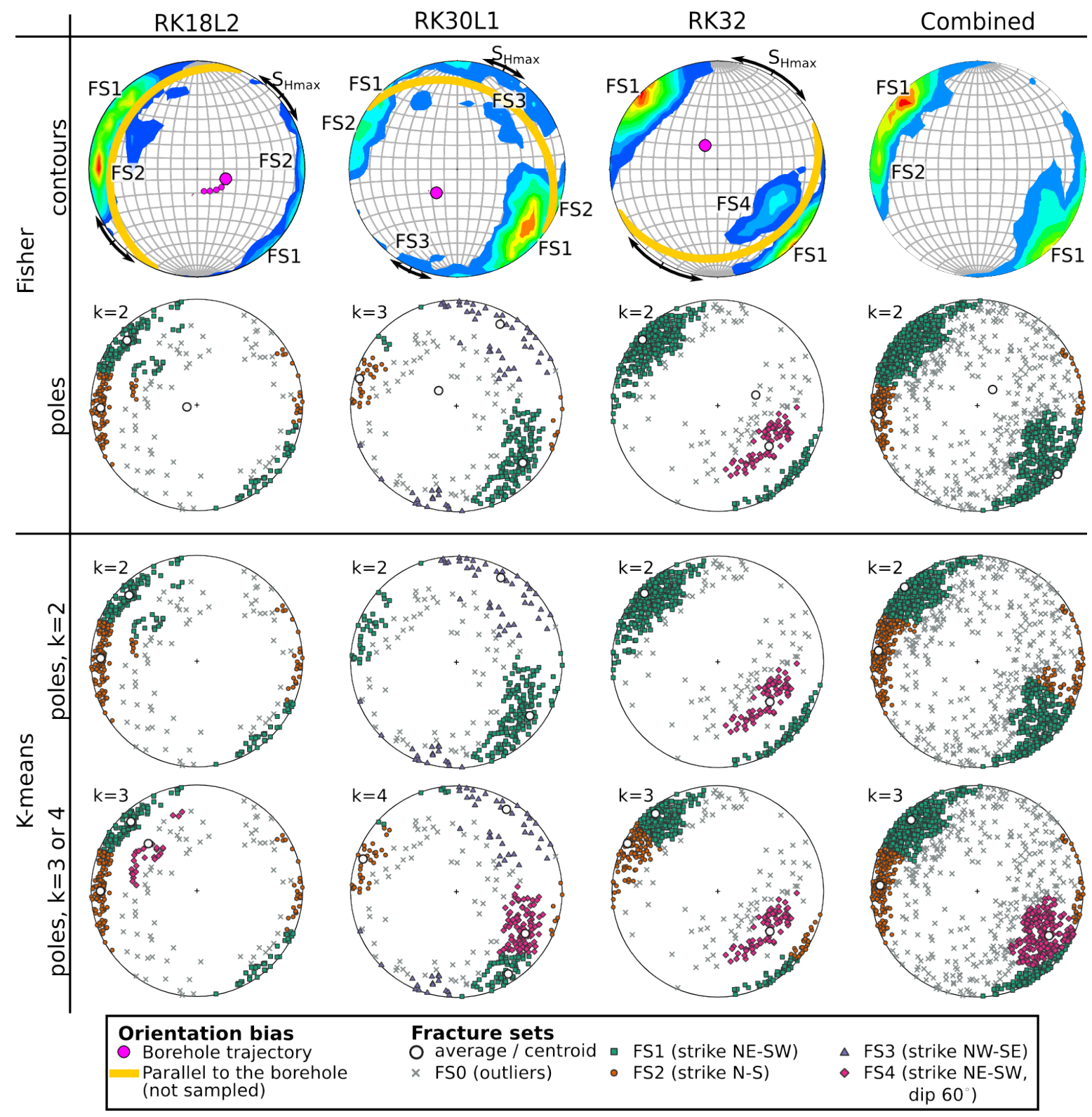

Figure 4.5: Fracture set orientations in the Rotokawa andesites for each of the three BHTV logs and for the combined boreholes. Top: Fisher contouring, $\mathrm{S}_{\text {Hmax }}$ orientation (mean value and one standard deviation; McNamara et al. 2015), and great circle with the orientation of poles to planes of fractures not sampled because they are parallel to the borehole (the pole of this great circle is the borehole trajectory); pole to planes separated into fracture sets. Bottom: pole to planes separated in fracture sets using clustering algorithms. $k$ : number of sets. All stereonets displayed as lower hemisphere Schmidt projection, Fisher contouring colours representing percentages. 
loci of poles to fractures parallel to each borehole not sampled by the BHTV log are presented as great circles on Figure 4.5. FS4 is considered to form a separate set from FS1 rather than stemming from an observation bias, because it contains a large number of fractures dipping $\sim 60^{\circ}$. Each fracture set is well clustered, with high $(\geq 0.9)$ cluster intensity (measured by the resultant of the mean vector which varies from 0 for a non-clustered set, to 1 for a single orientation). The spacing of outliers $(<1 \%$ Fisher density) is not analysed as these fracture do not have a mean orientation.

K-means clustering, supported by fuzzy K-means and agglomerative clustering, indicate that configurations with two clusters, and with either three or four clusters, are possible for each borehole (Figure 4.5). In addition to the sets delineated using Fisher contours, FS2 is separated from FS1 in borehole RK32, and FS4 from FS1 in boreholes RK18L2 and RK30L1. Although the separation of FS1 and FS4 in borehole RK18L2 occurs in a direction most affected by the sampling bias, the delineation of the FS4 set in the two other boreholes supports its existence.

\subsubsection{Spacing}

Fracture spacing varies between 0.01 and $165.03 \mathrm{~m}$ with an average of $3.4 \mathrm{~m}$ and a median of $1.1 \mathrm{~m}$. The highest AIC ranking distribution for fracture spacing within truncation limits $(0.03-30 \mathrm{~m})$ is log-normal for FS1; pareto or power-exponential for FS2; gamma or log-normal for FS3; and either pareto, gamma, or log-normal for FS4 in boreholes RK32, RK30L1, and the combined dataset, respectively (Table 4.A.2). The shapes of the cumulative density function (CDF) of fracture spacing on log-log coordinates reflect the differences evaluated with the AIC: the CDF of FS1 and FS3 are curved (Figure 4.6a, c), whereas the CDF of FS2 and FS4 have straight portions characteristic of power-law distributions (Figure $4.6 \mathrm{~b}, \mathrm{~d}$ ).

Depending on the clustering configuration adopted (i.e., Fisher density contours, or K-means with two or more clusters), the AIC condition is also satisfied by alternate distributions (Table 4.A.2). Subsets with fewer than 50 data points have four or five distributions satisfying the AIC condition because data is under-constrained (Chapter 3), and are thus of low confidence. In addition, nearly all $\hat{\sigma}$ parameters of the powerexponential distributions have large (>1000 units) confidence intervals for subsets of FS2 and FS4 orientations (in each borehole and combined), and are thus not well constrained. For FS2 in RK18L2 with a 4-clusters configuration, where power-exponential is the only distribution satisfying the AIC condition but with a rather large $\hat{\sigma}$ confidence interval (248 units), pareto and log-normal are the next lowest AIC distributions $(\Delta(\mathrm{AIC})=16$ and 17 , respectively), as with the other FS2 subsets. In summary, although less well-fitting than log-normal (for FS1) and pareto (for FS2) distributions, the pareto and power-exponential distributions are also good contenders for FS1 in boreholes RK18L2 and RK30L1, and log-normal for FS2. 

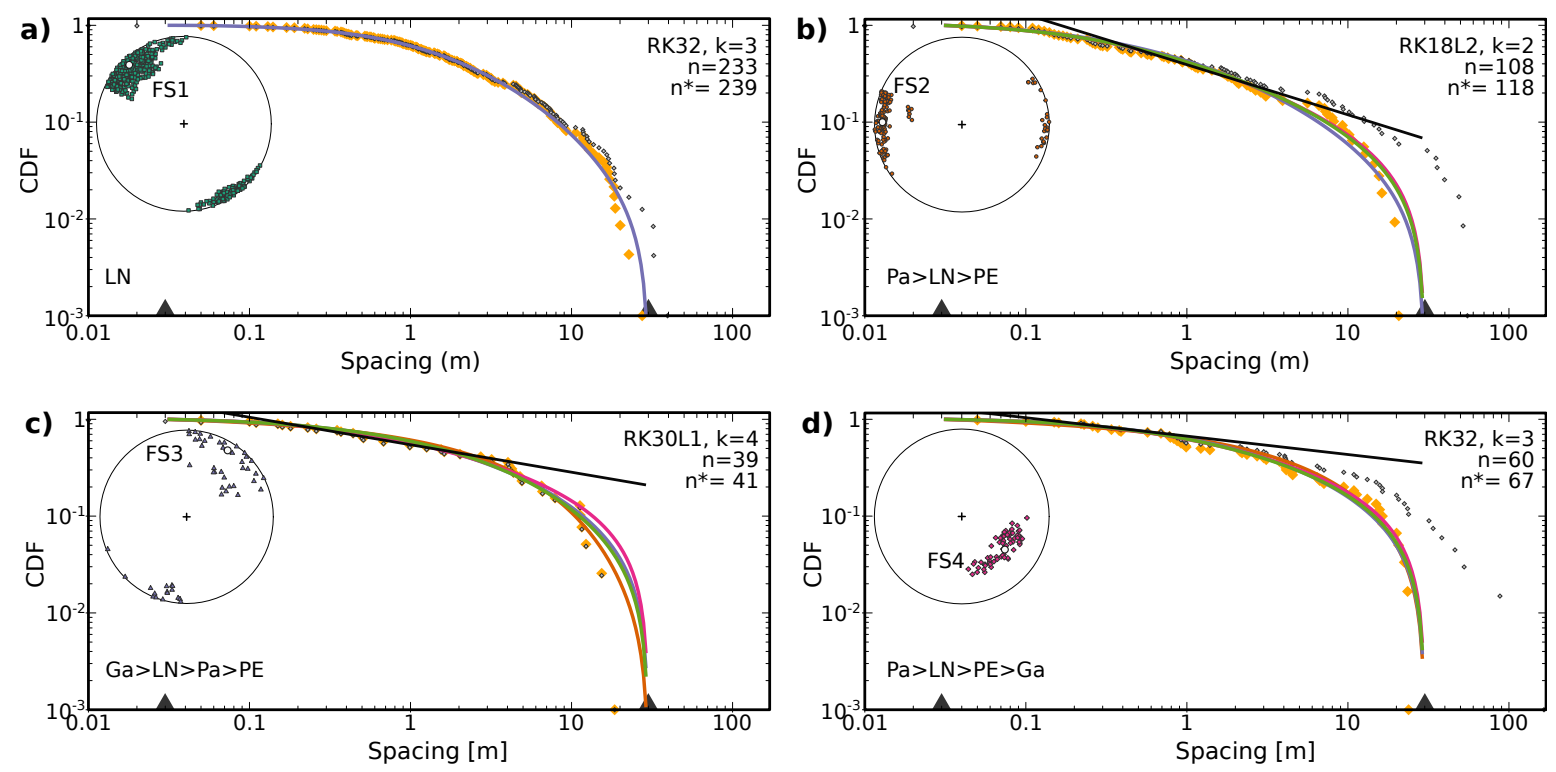

- Truncation limits

Distributions

- Data

- Gamma (Ga)

- Log-normal (LN)

- Power-exponential (PE)

- Pareto (Pa)

- Pareto-derived power-law

Figure 4.6: Examples of cumulative density function (CDF) and probability distributions satisfying the AIC condition for the spacing of each fracture set, listed by decreasing AIC rank. a) FS1 in borehole RK32 with 3 clusters. b) FS2 in borehole RK18L2 with 2 clusters. c) FS3 in borehole RK30L1 with 4 clusters. d) FS4 in borehole RK32 with 3 clusters. The exponential distribution never satisfies the AIC condition and is not displayed. All displayed subsets are delineated with K-means clustering. The parameter values of the highest ranking distribution are listed in Table 4.A.2. $k$ : number of clusters; $n$ : number of fracture within truncation bounds used for the fitting; $n^{*}$ : total number of fractures (without truncation). 
The parameters of the log-normal distribution fitting FS1, where it has the highest ranking, are very similar for each borehole and the combined dataset, with small $95 \%$ confidence intervals (Table 4.A.2). The power-law coefficient $(\hat{\alpha})$ of FS2 in boreholes RK18L2 and the combined dataset, where these distributions have the highest ranking, varies between 1.2 [1.1-1.4] (most likely value and 95\% confidence interval) for the combined dataset and 1.6 [1.4-2.0] for RK18L2. The lack of small spacing for FS2 $(<0.1 \mathrm{~m})$, which favoured an power-exponential distribution with the 4-clusters configuration, suggests a high minimum bound of the power-law exponent (Figure 4.6b). The power-law coefficient is not constrained for borehole RK30L1 ( $<25$ data points). For FS4, the power-law coefficients vary between 1.2 [1.0-2.8] in RK32 to 2.0 [1.4-3.5] for RK30L1, and is not constrained for RK18L2 which has only 11 points.

The coefficient of variation $(\mathrm{Cv})$ can indicate if the dataset is clustered, and is calculated for normal distributions as the ratio of the spacing standard deviation to the mean spacing (Gillespie et al., 1999; McCaffrey et al., 2003). At the Rotokawa Geothermal Field, Cv varies between 1.2 and 1.9 within truncation limits, without clear correlation between fracture sets, which indicates that the fractures are slightly clustered. The $\mathrm{Cv}$ calculated for log-normal distributions (Limpert et al., 2001) is $\geq 2$ for all fracture sets, with a higher mean Cv for FS2 and FS4 (10 and 6.5, respectively) than for FS1 and FS3 (3 and 6, respectively). Although the precise $\mathrm{Cv}$ is dependent on the assumed underlying distribution (i.e., normal or log-normal), both sets of calculations suggest that fracture spacings are clustered.

\subsubsection{Thickness}

Fracture thicknesses in cores vary between 0.5 and $3 \mathrm{~mm}$, with a mean of $1.2 \pm 0.5 \mathrm{~mm}$ (one standard deviation). Overall, $70 \%$ of fractures in cores appear to be fully infilled, while the remaining $30 \%$ have openings of $0.2-2 \mathrm{~mm}$, which usually varies along the fracture plane.

Fracture thickness in the BHTV logs varies between 1 and $57 \mathrm{~mm}$, with an average of $11 \pm 7 \mathrm{~mm}$. Exponential distributions have the highest SBC ranking for fracture thickness evaluated within truncation limits $(9-30 \mathrm{~mm})$ for the combined dataset, each fracture set and each borehole, apart from borehole RK32 which is best approximated by a log-normal distribution (Table 4.A.1). There are no other close contenders for the combined dataset containing 597 data points. Other distributions satisfy the SBC condition for individual fracture sets: log-normal distribution for FS1; log-normal and gamma distributions for FS4; and all distributions for fracture set FS2 which contains the smallest number of data points (74) and is thus under-constrained.

The histogram of fracture thickness measured from BHTV logs appears as a straight line on a log-linear plot, in agreement with the highest SBC ranking of the exponential distribution (Figure 4.7). This histogram is normalised by the length of borehole 


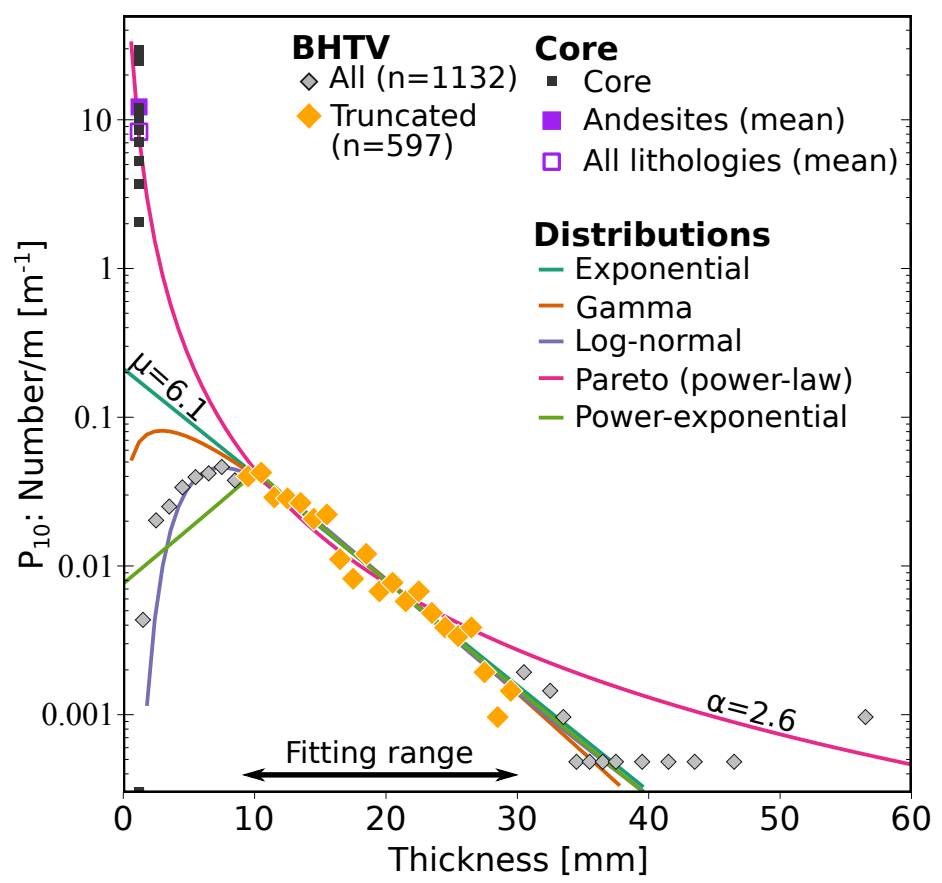

Figure 4.7: Histogram of the fracture thickness in BHTV logs and cores, normalised by the lengths of boreholes and cores, respectively, with probability distributions fitted to fracture thickness in BHTV logs between 9 and $30 \mathrm{~mm}$. The exponential distribution appears as a straight line in this coordinate system.

and shows the fitted distributions extrapolated beyond the truncation limits (Chapter 3). While the statistical analysis shows that the power-law distribution estimated from BHTV data is unlikely compared to the other distribution forms, the power-law distribution appears to fit the BHTV thickness data well between 9-30 mm, and also fits the core dataset.

The exponential coefficient $\hat{\mu}$ fitted to the fracture thickness in BHTV logs varies between 3.2 [2.7-3.9] $\mathrm{mm}$ in borehole RK18L2, and 8.6 [7.4-10.0] $\mathrm{mm}$ in borehole RK32 (for which the exponential distribution is not the most likely), with a median of $6.1 \mathrm{~mm}$ (Table 4.1). The $\hat{\sigma}$ parameter of the gamma distributions is often close to 1 (between 0.5 and 1.5), which makes it similar to an exponential distribution. The power-law coefficients $\hat{\alpha}$ vary between $1.9[1.6-2.2]$ and 4.3 [4.3-4.4] with a median of 2.6.

\subsubsection{Density}

The linear fracture density $\mathrm{P}_{10}$ varies between cores (e.g., low in Figure 4.3 and high in Figure 4.3b; Table 4.1), and over short length scales on the same core $(10-20 \mathrm{~cm}$, Figure $4.3 \mathrm{~b}$ ). There is no correlation between fracture density and alteration intensity (Figure 4.8). Amongst the few cores available, the $\mathrm{P}_{10}$ density in andesite breccias is lower $\left(2\right.$ to $\left.7.1 \mathrm{~m}^{-1}\right)$ than in the andesite lavas $\left(8.6\right.$ to $\left.29.3 \mathrm{~m}^{-1}\right)$, although a $0.7 \mathrm{~m}$-long core of andesite lava contains no discernible fractures. The core of rhyolite lava has a $\mathrm{P}_{10}$ density similar to those of andesite lavas. The two cores of tuff have low $\mathrm{P}_{10}$ of 


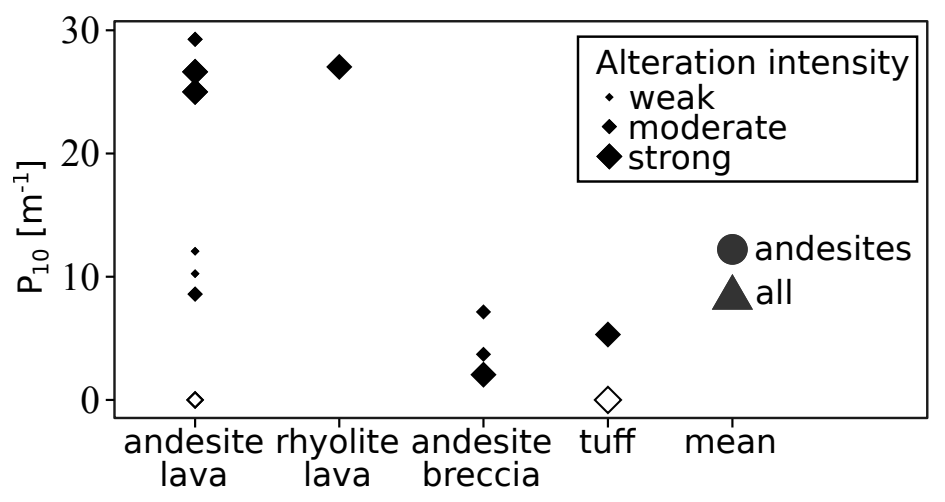

Figure 4.8: Linear fracture density $\left(\mathrm{P}_{10}\right)$ in cores as a function of the lithology and alteration intensity, for the uncorrected dataset. Open symbols are for cores without any fractures. The mean densities are weighted by the length of each core for both the andesitic lithologies and all lithologies together.

$5.3 \mathrm{~m}^{-1}$ in RK08, and no fractures in a $6.6 \mathrm{~m}$-long core in RK30L1. Fractures occupy 0-6.4\% of the rock mass ( $\mathrm{P}_{33}$ porosity), although the upper bound of this range occurs over a short core $\left(0.4 \mathrm{~m}\right.$-long). The average $\mathrm{P}_{33}$ in cores, weighted by the length of each core, is $0.9 \%$ for all lithologies, and $1.7 \%$ for just lavas and breccias. The $0.9-1.7 \%$ $\mathrm{P}_{33}$ values for cores is a minimum, as results corrected for orientation sampling bias suggests that there are more fractures in the rock mass than those observed $\left(7 \% \mathrm{P}_{33}\right.$ in lavas and breccias).

The mean $\mathrm{P}_{10}$ in BHTV logs is $0.5 \mathrm{~m}^{-1}$ and varies slightly between boreholes (Table 4.1). The mean $\mathrm{P}_{33}$ of $1.5 \%$ for BHTV logs (weighted by the length of each borehole) is also larger when corrected for orientation bias (mean of $5.4 \%$ ).

\subsection{Discussion}

\subsubsection{Spacing and Orientation}

The spacings of fracture sets of different orientation are best approximated by different distribution forms. Log-normal spacing of fracture sets FS1 (NE-SW strike) and FS3 (NW-SE strike), with similar fitting parameters (Table 4.A.2), indicates a characteristic scale that may reflect the predominance of internal effects (e.g., thermal, chemical or mechanical) on fracture generation (Bonnet et al. 2001, Figure 4.1 a). By contrast, the highest ranking distribution for spacings of sets FS2 (N-S strike) and FS4 in borehole RK32 (NE-SW strike, dipping $\sim 60^{\circ}$ ) is power-law and thus scale-independent, with coefficients similar to those reported by Gillespie et al. (1993) for faults. Thus, spacing of these subordinate fracture sets is likely controlled by rupture processes driven by external tectonic forces (Bonnet et al., 2001, Figure 4.1b). While only three major subsurface faults have been identified with confidence in the Rotokawa Geothermal Field, the presence of other subsurface active faults has been inferred from localised 
$\mathrm{S}_{\text {Hmax }}$ direction rotations observed in BHTV logs (McNamara et al., 2015), and may cause this power-law spacing distribution locally.

Characteristic scales of log-normal fracture spacing in andesite reservoirs may result from stratification of the volcanic andesitic sequence, the finite dimensions (width, thickness, length) of each lava flow, and the original jointing and flow-banding formed during cooling and emplacement of the lavas. In volcanic outcrops, auto-breccias forming the external envelope of lava flows typically have high porosity and act as a mechanical interface, confining columnar joints within each lava flow (DeGraff and Aydin, 1987; Hetényi et al., 2011, Chapter 2). Together with hydrothermal breccias (Rae, 2007), lava-flow margins may form mechanical interfaces within the Rotokawa reservoir. Although stratification of the Rotokawa andesites is not directly observed, log-normal spacing distributions have also been found for veins in andesite-hosted epithermal gold deposits (at Waihi, located $140 \mathrm{~km}$ north of Rotokawa Brathwaite et al., 2001; Spörli and Cargill, 2011); and Hishikari in Japan (Sasaki, 2006)). Such epithermal deposits can be considered to some extent as fossil equivalents to active geothermal fields Rowland and Simmons, 2012; Simpson et al., 2015). In the Waihi andesites, some veins tend to follow lava flow cooling joints, especially when the lavas are well-jointed (Brathwaite et al., 2001), similarly to thin dykes that partly follow cooling joints in lava flows Gudmundsson, 2011).

Flow-banding in Rotokawa andesites observed in places (McNamara et al., 2016a, and references therein) may control fracture formation, such as in core RK04 where fractures are aligned with the vertical flow-banding (Figure $4.3 \mathrm{~d}$; RK04 borehole is vertical). In New Zealand epithermal deposits, flow-banding influences the emplacement of hydrothermal breccias at Onemana (Zuquim and Rowland, 2013) but has only minor effects on vein emplacement at Waihi where strong silicification has homogenised the rock (Brathwaite et al. 2001$)$. In the Rotokawa andesites, hydrothermal alteration may mechanically homogenise the volcanic sequence in places, but breccias and flowbanding may still produce mechanical interfaces which influence fracture propagation Misra et al. 2015). The Rotokawa andesitic sequence as a whole may thus behave mechanically like sedimentary layered systems, in which non-power-law joint spacing distributions are observed (Figure 4.1 a, Ladeira and Price, 1981, Schöpfer et al., 2011).

Overall, fracture spacing results in the Rotokawa reservoir suggest the superposition of (1) a pervasive network of fractures (FS1 and FS3) influenced by the host rock stratification, and (2) subordinate fracture sets (FS2, FS4) whereby fracture location is controlled by active faulting. A similar combination of exponential fracture spacing for host rock (with also has a characteristic scale), and power-law spacing for fault rock, has been documented in outcropping layered carbonates associated with normal and strike-slip faults (Agosta et al. 2010). The vein distribution at Waihi is also inferred to have been controlled both by the initial jointing of the lava, and by subsequent faulting events, especially close to faults where the lithological controls are overridden 
by tectonic controls (Brathwaite et al., 2001, Spörli and Cargill, 2011).

The general fracture orientations in the Rotokawa reservoir are controlled by tectonic stresses (McNamara et al., 2015). The dominant fracture set FS1, and subordinate set FS4, strike parallel to kilometre-scale normal faults and the contemporary $\mathrm{S}_{\text {Hmax }}$ direction, with high dip magnitudes consistent with the normal faulting regime. A similar dominant tectonic control on vein orientation is observed at Waihi (Brathwaite et al., 2001; Spörli and Cargill, 2011). The subordinate FS3 fracture set, observed only in RK30L1 borehole, may be related to inferred accommodation zones between rift segments (Rowland and Sibson, 2001) or to relays or bends in fault surfaces linking NE-SW-striking fault segments (Childs et al., 2009). Fractures of FS2 orientation may be related to $\mathrm{N}-\mathrm{S}$-striking structures related to the Hauraki Rift, terrane sutures inferred in the Mesozoic greywacke basement, or fault linkage structures Rowland and Sibson, 2004; Seebeck et al., 2014a; McNamara et al., 2015).

\subsubsection{Thickness}

Fracture thickness in BHTV logs is best approximated by an exponential distribution. The highest AIC rank of log-normal distribution in borehole RK32 is interpreted to reflect a sampling effect, with a possible higher left-truncation limit than in other boreholes. Conversely, the core dataset, with fracture thicknesses one order of magnitude smaller than those observed from BHTV logs, matches the power-law distribution obtained from the BHTV data (Figure 4.7). Three scaling relationships can explain these observations: either $(1)$ the fractures at core $(\sim 1 \mathrm{~mm}$-thick $)$ and BHTV $(\sim 10 \mathrm{~mm}$ thick) scales are related, and follow the same power-law distribution; or (2) the BHTV dataset is approximated by an exponential distribution and is not related to the core dataset; or (3) the thickness in BHTV logs and cores follow the same exponential distribution, but the BHTV logs detect only $2 \%$ of the fractures intersecting the borehole.

Case 1 is the most likely, i.e. fracture thicknesses observed on cores and BHTV logs follow the same power-law distribution. Distribution fitting of datasets spanning only half an order of magnitude is not well constrained and explains why the SBC favours the distribution with only one parameter (exponential) rather than those with more parameters (including pareto). In addition, the power-law distribution is the only one able to explain the occurrence (although rare) of fractures $>40 \mathrm{~mm}$-thick on the BHTV logs, above the truncation limit of the data. While some of these apparently thick fractures observed on BHTV logs may contain several thin fractures not resolved individually, $>40 \mathrm{~mm}$-thick fractures have been observed in outcrop of epithermal deposits (Brathwaite et al., 2001), and their presence within the Rotokawa andesites cannot be ruled out.

Case 2 is not supported by outcrop observations at Waihi, where vein thickness correlates positively with proximity to the main fluid source (Brathwaite et al., 2001), 
rather than to a change of thickening processes between different scales. Similarly, measurements of fracture apertures at different and overlapping scales in mudstones and dolomites in the Sorrento Peninsula (Italy) are best modelled by a single powerlaw distribution (Guerriero et al., 2011). Case 3 is also unlikely. Comparison of cores and BHTV logs in the Soultz geothermal field showed that $1 \mathrm{~mm}$-thick sealed fractures abundant in cores were beyond resolution of the BHTV logs, and this detection issue can mostly explain the difference of fracture density between the two datasets Genter et al., 1997). Similar resolution limits of fracture thickness in BHTV logs have been inferred by Barton and Zoback (1992) at Cajon Pass (U.S.A.). These observations do not suggest significant undersampling of 9-30 mm-thick fractures in BHTV logs, although direct comparison of core and BHTV log over the same intervals of the Rotokawa Geothermal Field would be needed to test this assumption.

Power-law coefficients of fracture thickness in Rotokawa BHTV logs (2.6-3.1) are similar to the coefficients derived from BHTV log data for geothermal fields hosted in faulted crystalline formations (Barton and Zoback, 1992, Radilla et al., 2012), and in andesites based on outcrop scanline measurements (McCaffrey et al., 2003). Fault zone thicknesses $(0.01-\sim 110 \mathrm{~m}$-thick) measured in tunnels in the south of the TVZ are also approximated by a power-law distribution, with a coefficient of 1.5 and 1.8 in Miocene sandstone and mudstone, respectively (Seebeck et al., 2014b). While these latter coefficients are lower than those found in the Rotokawa Geothermal Field, they support the interpretation that fracture thickness measurements from core and BHTV logs in the Rotokawa andesites follow a power-law distribution at the measured scales, and may extend to larger fracture sizes.

\subsubsection{1-D and 3-D Fracture Abundances}

The core and BHTV log data sample fracture thicknesses at two orders of magnitude: $1-3 \mathrm{~mm}$ in cores, and 2-57 mm in BHTV logs. Thin-section analysis by Siratovich et al. (2014) provides $\mathrm{P}_{10}$ and $\mathrm{P}_{32}$ measurements of $\sim 5 \mu \mathrm{m}$-thick fractures, and we derived $\mathrm{P}_{33}$ assuming a $5 \mu \mathrm{m}$ mean fracture thickness (Figure $4.9 \mathrm{~b}$ ). The power-law distribution approximating both core and BHTV log fracture thicknesses largely over-estimates the linear density $\left(\mathrm{P}_{10}\right)$ observed for thin-sections (Figure $4.9 \mathrm{a}$; additional tests with raw $\mathrm{P}_{10}$ also yield an overestimation of thin-section data). Fractures at thin-section, core and BHTV log scales occupy a similar percentage of rock volume $\left(\mathrm{P}_{33}\right)$, with an average of $4.1 \%, 7 \%$ and $5 \%$, respectively (Table 4.1 , Figure $4.9 \mathrm{~b}$ ).

The $\mathrm{P}_{10}$ measurements imply that fracture generation processes differ between the thin-section $\left(10^{-6} \mathrm{~m}\right.$-thick) and core $\left(10^{-3} \mathrm{~m}\right.$-thick) scales. A number of core and BHTV log intervals do not display any fractures, in contrast to the pervasive presence of fractures in available thin-sections (most of the studied samples in Siratovich et al. (2014) did not have distinct macrofractures, and include samples from core RK27L2\#1 


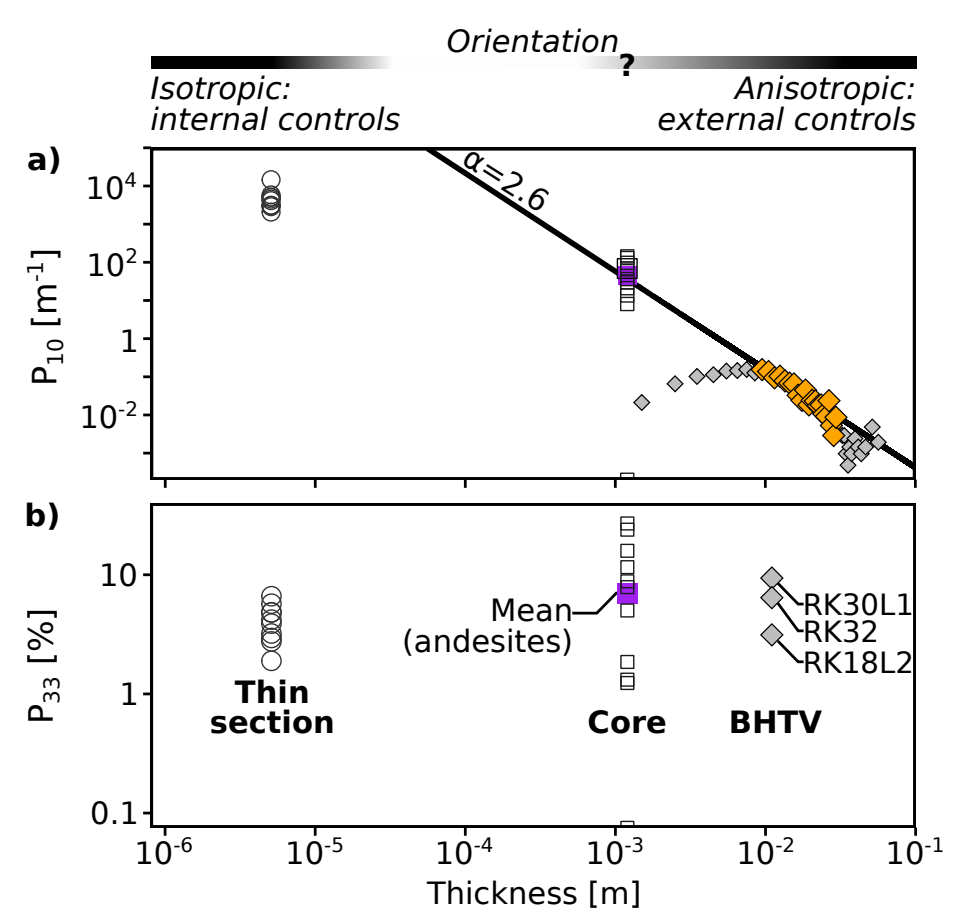

Figure 4.9: Fracture abundance for fractures in thin-sections (Siratovich et al., 2014), cores, and BHTV logs (Terzaghi-corrected datasets). a) Linear density $\mathrm{P}_{10}$. b) Percentage of fracture volume within the rock mass $\left(\mathrm{P}_{33}\right)$. The thin-section values are plotted assuming a fracture thickness of $5 \mu \mathrm{m}$, also assumed to calculate $\mathrm{P}_{33}$ values. The symbology for core and BHTV log data is the same as in Figure 4.7.

which has a low $\mathrm{P}_{10}$ at core scale). Most importantly, fractures observed in thin-sections have an isotropic orientation, and have been interpreted as being controlled by a uniform thermal stress experienced during the initial lava formation, burial, hydrothermal alteration or drilling (Siratovich et al., 2014). While shear displacement of crystals within the lavas has been observed at thin-section scale (e.g., Ramirez and Rae, 2009), it is rare. The isotropic orientations of fractures at thin-section scales contrast with the dominant anisotropic orientation of fractures in BHTV logs and reservoir-scale faults which are strongly controlled by tectonic stresses. Although it is not clear whether fractures in cores have preferential orientations, due to the short length of available core fragments, the presence of slickenslides on some fracture faces suggests that these fractures are, at least in part, controlled by tectonic stresses. Thus, the change between predominantly internal (thermal) and external (tectonic/lithologic) controls on fracture orientation and density, potentially associated with fracture coalescence and interactions, occurs at thickness scales intermediate between $10^{-6}$ and $10^{-3} \mathrm{~m}$. However, the similar $\mathrm{P}_{33}$ at all three scales of observation suggests a self-similar fracture proportion of rock volume in 3-D. 


\subsubsection{Effect of Lithology and Alteration on Fluid Pathways}

The apparent lack of correlation between fracture density and alteration intensity observed in the limited cores available is consistent with results from microstructural measurements and laboratory experiments on samples of Rotokawa andesites (Siratovich et al., 2014). Indeed, the $\mathrm{P}_{32}$ values for fractures in thin-sections appears to be independent of the alteration and mineralogy of the studied specimens. In addition, although the strength of the andesites decreases as the intensity of the alteration increases, brittle behaviour still occurs in altered rocks under the pressure and temperature conditions representative of the Rotokawa reservoir Davidson et al., 2012, Siratovich et al., 2014, 2016).

Some rhyolite breccias are (or are inferred to be) permeable in TVZ geothermal fields (e.g. Ohaaki (Mroczek et al., 2016) and Wairakei (Milloy and Lim, 2012)). In the Rotokawa Geothermal Field, fracture density in cores is higher in lavas than in breccias. In breccias, the numerous veins observed surrounding clasts suggest that fluids can preferentially flow through open spaces along clast boundaries, rather than through newly generated fractures in these high-strength rocks (60 to $211 \mathrm{MPa}$ unconfined compressive strength; Siratovich et al., 2014; Wyering et al., 2014). Therefore, permeability in breccias relies on the inter-connections of void space between clasts, and between clasts and rare fractures. The resulting fluid flow paths along clasts are more tortuous than those along sub-planar fractures, and breccias with plugged pores potentially have lower permeability than fractured lavas as a result. It is unclear, however, how quickly the porosity between clasts becomes plugged by mineral precipitation, which impacts how much, and for how long, breccias can host permeability.

While tuff lithologies are not part of the main reservoir at the Rotokawa Geothermal Field, they affect shallow fluid circulations and connections between surface and reservoir (Rae, 2007). The two cores of tuff studied here have low fracture density, including the RK30L1 core in which no fracturing was observed. This $6.6 \mathrm{~m}$-long core is strongly altered and has a porosity of $25-35 \%$ (Rae et al., 2010), which is significantly higher than the typical porosity of $<10 \%$ reported for andesites Siratovich et al. 2014). In partially welded and strongly altered tuff lithologies, fluid flow is thus likely controlled primarily by matrix porosity rather than by fractures, even when strongly altered, as inferred at the Mokai and Ohaaki Geothermal Fields, TVZ (Bignall et al., 2010; Mroczek et al., 2016).

\subsubsection{Potential Controls on Structural Permeability}

Identifying which fracture characteristics control permeability is not straightforward in lava-hosted reservoirs where the fracture system geometry results from a combination of lithological, thermal, and past and present-day tectonic processes. For example, 
at the Karaha-Telaga Bodas Geothermal Field, Indonesia, permeable fractures are not the widest and are not necessarily located within high fracture density zones, but are roughly perpendicular to $\sigma_{3}$ (minimum principal stress) and prone to slip under the modern stress regime (Nemčok et al., 2004). Similarly, permeable zones in the Rotokawa reservoir contain fractures from each fracture set, some of the thickest fractures, some high-density fracture clusters, and some local in-situ $\mathrm{S}_{\mathrm{Hmax}}$ rotations inferred to be associated with slip on NE-SW and E-W-striking active faults, but no one-to-one correlation between permeability and fracture attribute has been observed (McNamara et al. 2015).

Critically stressed faults of high $(>0.6)$ shear/normal stress ratio are generally considered to be dilatant and hydraulically conductive Barton et al. 1995; Townend and Zoback, 2000; Barton et al., 2013). Preliminary work on the Rotokawa stress field suggests that fractures of FS1 and FS4 orientations, and of FS2 orientation in specific stress ratio configurations, have high shear/normal stress ratio (Davidson et al., 2012). Further work is needed to further explore this correlation, or lack thereof, between high shear/normal stress ratio and permeability.

Fracture connectivity, controlled by fracture density, length Bour and Davy, 1998; Bonnet et al., 2001) and orientation (Kissling et al., 2015b), can cause large variations in reservoir permeability at short scales (smaller than fracture length). In layered carbonate outcrops, Agosta et al. (2010) established a correlation between hydrocarbon flow and fracture connectivity, rather than density. In the Rotokawa reservoir, intersections between the pervasive network of fractures of FS1 orientation, with faultcontrolled fractures of FS4 (and partly FS2) orientations in parts of the reservoir, may thus concentrate fluid flow.

In combination with a power-law distribution for fault thickness, Seebeck et al. (2014b) documented a power-law relationship of fluid flow rates, where few 'golden' thick, connected faults conduct the bulk of the fluids. Such a configuration in the Rotokawa andesites can also explain the observed compartmentalisation, with closelylocated boreholes of high and low fluid productivity (Hernandez et al., 2015) which would intersect, or not, one of these highly conductive faults. Flow models based on geometric fracture models at reservoir scale would help constrain the respective roles of fracture system geometry, stress and water/rock interactions in controlling permeability. While only a subset of the fractures described in this chapter are permeable, a fracture and flow model of the Rotokawa reservoir would have to accommodate the fracture geometries described in thin-section, cores and BHTV logs.

\subsubsection{Modelling Rotokawa Fracture Systems at Reservoir Scale}

Fracture orientation, spacing, thickness and abundance reported in this chapter can be used to generate or constrain geometrical fracture models. The power-law thickness 
distribution evaluated at core- and BHTV log-scales may be extrapolated to predict the potential number of thicker fractures $(>40 \mathrm{~mm})$ and faults not resolved by the available reservoir data. The number of faults recognised in a reservoir typically increases as new wells are drilled in different parts (or depth) of the reservoir (Massiot et al., 2011), so it is likely that additional faults exist between current boreholes in the Rotokawa Geothermal Field. Extrapolating from borehole- to field-scale must, however, be done with caution. Power-law distributions of fault sizes tend to have lower exponents when determined at seismic-scale compared to outcrop-scale, possibly due to a deviation from a power-law relationship, or to different fault growth processes at different scales $\sqrt{\mathrm{Nicol}}$ et al., 1996). For example, large faults may be controlled by the average mechanical properties of the sequence in which they develop rather than by smaller-scale variations.

Fracture length cannot be measured on cores, nor on the available BHTV logs. Probabilistic methods based on the proportion of fractures partially and fully intersecting the borehole $(\ddot{O} z k a y a, 2003)$ require full azimuthal coverage of the borehole on the BHTV log, which is rarely the case for the available logs acquired in the Rotokawa Geothermal Field. Evolving fracture models (Davy et al., 2013) may constrain the fracture length distributions, although lithological controls on fracture growth would need to be included in the modelling. Outcrop-based analysis of fracture length in volcanic sequences (such as Gudmundsson, 2000), and near faults (e.g., Vermilye and Scholz, 1995, Johnston and McCaffrey, 1996) may be used to model fracture sets FS1/FS3 and FS2/FS4, respectively.

The choice of fracture aperture distribution and associated transmissivity is crucial for fluid flow and thermal modelling in geothermal systems. For example, numerical experiments demonstrate that a log-normal fracture aperture distribution channelises heat depletion and hastens thermal breakthrough, while a constant aperture distribution uniformly extracts heat from the system (Doe et al., 2014). In the Rotokawa Geothermal Field, only $30 \%$ of fractures in cores have an opening discernible with the naked eye, and in most cases this opening is not constant along the fracture plane, so the results presented here for fracture thickness cannot be directly related to fracture aperture. In addition, fracture aperture is sensitive to fracture roughness Ishibashi et al., 2014) and confining pressure (Chen et al., 2000; Barton et al., 2013). Given the uncertainty of fracture length and hydraulic aperture, future fracture and flow numerical models should reflect these uncertainties.

\subsection{Conclusion}

The statistical analysis of fracture attributes at several scales of observations constrains the geometry of the Rotokawa fracture system, which is a necessary input into geologically-informed reservoir models and improved understanding of fluid pathways 
in such andesite-hosted geothermal systems. Fracture spacing and density measured at several scales of observation suggest a combination of tectonic, lithological and thermal controls on the geometry of the fracture system. Andesite breccias and flow-banding within lavas affect fracture location, density and tortuosity, and hence the connectivity of the system. The dominant NE-SW-striking fracture population orientation is controlled by recent and contemporary stresses in the Taupo Rift, while inherited basement structures or fault linkage may also influence N-S and NW-SE-striking subordinate fracture orientations. At the BHTV log scale, the dominant fracture populations have a scale-dependent log-normal fracture spacing, interpreted to be related to stratification within the lava sequence, individual lava flow size, or inherited jointing. In contrast, scale-independent power-law spacing of subordinate fracture populations are interpreted to be associated with tectonic faults. Fracture thickness is approximated by a power-law distribution spanning 1.5 orders of magnitude (1-40 mm-thick), which may extend to larger fault sizes (100 m-thick). However, this power-law does not continue to thin-section scale $(\sim 5 \mu \mathrm{m})$ fractures, reinforcing previous interpretation that these pervasive fractures with isotropic orientations could be controlled by thermal rather than lithologic or tectonic stresses. However, fracture volumes of $\sim 5 \%$ of the rock mass are similar at thin-section, core and BHTV log-scales, suggesting a self-similar fracture volume behaviour in $3-\mathrm{D}$.

\section{Acknowledgments}

We would like to thank the Rotokawa Joint Venture (Mercury New Zealand and Tauhara North No.2 Trust) for providing reservoir data and allowing publication of this work. 


\section{Appendix}

\section{A Results of Probability Distribution Analysis}

Tables 4.A.1 and 4.A.2 present the results of the statistical analysis of fracture thickness and spacing. 


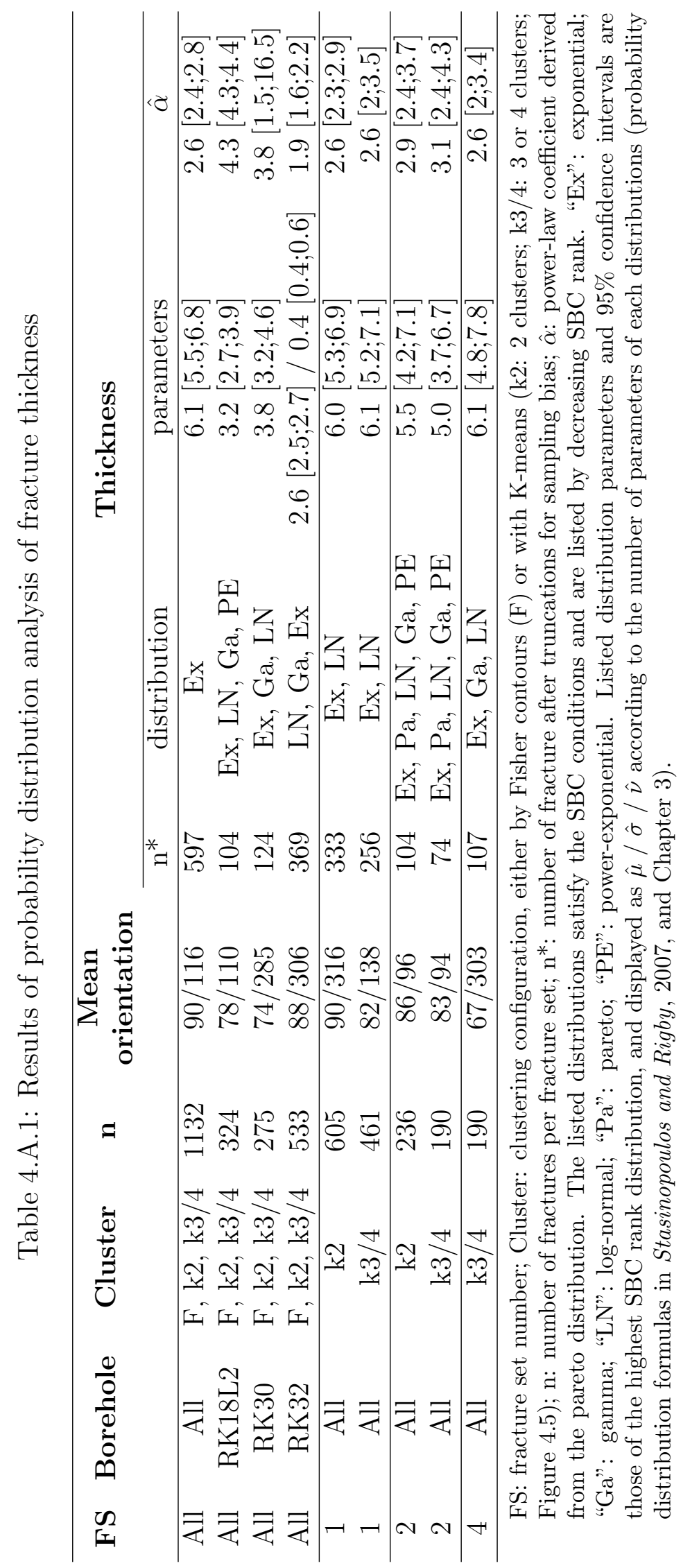




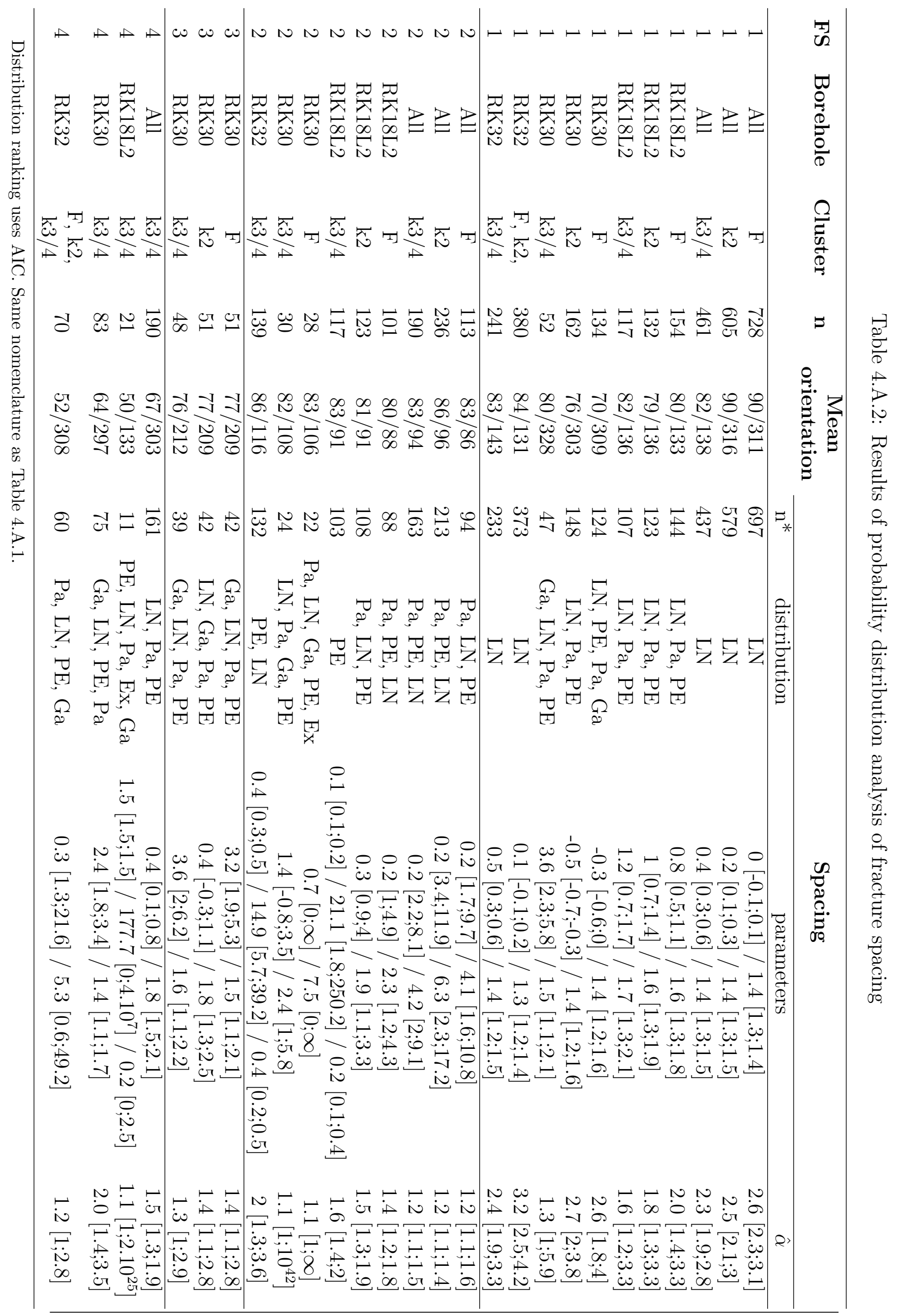




\section{The Alpine Fault Hangingwall Viewed From Within: Structural Analysis of Acoustic Image Logs in the DFDP-2B Borehole, New Zealand ${ }^{1}$}

\subsection{Abstract}

The Alpine Fault is the major dextral transpressive fault forming the Australia-Pacific plate boundary in the South Island of New Zealand. Acoustic borehole televiewer (BHTV) logs acquired in the DFDP-2B borehole late 2014 yield the first continuous, sub-surface description of the transition from schist protolith to mylonite in the hangingwall of the Alpine Fault, to a depth of $814 \mathrm{~m}$ below surface. Three feature sets are delineated. One set, comprised of foliation and foliation-parallel veins and fractures, has a constant orientation, with an average dip direction of $145^{\circ}$ parallel to the regional Alpine Fault trace, and an average dip magnitude of $60^{\circ}$ similar to nearby outcrop observations of metamorphic foliation in the mylonites. We interpret this foliation orientation as possibly being parallel to the Alpine Fault plane at depth $(\sim 1 \mathrm{~km})$ in the Whataroa valley. The other two auxiliary feature sets are interpreted as joints based on their morphology. One set occurs dominantly above $\sim 500 \mathrm{~m}$, comprises sub-vertical fractures with NW-SE $\left(137^{\circ}\right)$ strike, which are interpreted as joints that formed during the exhumation and unloading of the Alpine Fault's hangingwall. The other set is predominantly observed below $\sim 500 \mathrm{~m}$ and contains sub-horizontal fractures, that we interpret as inherited hydrofractures. Subsidiary fault zones, with brecciated textures similar to those observed in the nearby DFDP-1 boreholes, are identified at several depths. Some of the foliation-parallel fractures and the two subsidiary joints sets are likely permeable, individually and where they intersect each other. The change of subsidiary fracture orientation from sub-vertical to sub-horizontal at $\sim 500 \mathrm{~m}$ likely affects fluid flow directions in the hangingwall of the Alpine Fault.

\footnotetext{
${ }^{1}$ This chapter was primarily written by me with assistance and editorial input from my co-authors, in particular Tim A. Little. I aided Mai-Linh Doan, Bernard Célérier, Doug R. Schmitt, and the wireline technicians during data acquisition. I calculated the caliper values with Mai-Linh Doan. I conducted the bulk of the BHTV log processing, aided by David D. McNamara and Bernard Célérier. Interpretation of the data was completed with advice from the co-authors mentioned above and from John Townend, Virginia G. Toy and Rupert Sutherland, principal investigators of the DFDP-2 project.
} 


\section{$5.2 \quad$ Introduction}

Direct sub-surface measurements of structural, petrophysical, and hydrological properties near the Alpine Fault, the major plate boundary between the Pacific and Australian plates in the South Island of New Zealand, have previously been limited to shallow tunnels (Cox et al., 2015) and boreholes (Sutherland et al., 2012). These shallow observations do not necessarily represent conditions at deeper depth due to surface processes such as stress release or weathering. Drilling in the vicinity of active faults remains the only way to evaluate the in-situ conditions in which earthquakes are generated and damage zones develop (Zoback et al., 2007). One of the objectives of the Deep Fault Drilling Project (DFDP) is to study the state of the crust near the Alpine Fault, by drilling boreholes and sampling petrophysical properties (Townend et al., 2009). As part of the second phase of the DFDP project, the DFDP-2B borehole was drilled in late 2014 down to $818 \mathrm{~m}$ below surface in the Whataroa valley, central Southern Alps, in the hangingwall of the Alpine Fault (Figure 5.1).

A series of borehole geophysical logs was acquired in DFDP-2B between 264-893 m (measured along the borehole) in ductily-deformed schists (Sutherland et al., 2015). Wireline logging records petrophysical parameters continuously along the borehole (Rider, 1996), providing an intermediate scale of observation between laboratory and seismic experiments (Jeppson and Tobin, 2015; Boness and Zoback, 2006). Acoustic borehole TeleViewer (BHTV) logs provide oriented images of the inside of the borehole, by measuring the acoustic travel-time between the tool and the borehole wall, and formation acoustic impedance. BHTV log interpretation allows the systematic documentation of planar fabrics and structures intersecting the borehole (bedding, foliation, fractures, veins...) together with their orientation and morphological characteristics, stress-induced features when present, and of the acoustic impedance of the host formation (Zemanek et al. 1970). In the absence of drill cores in DFDP-2B, the interpretation of the BHTV logs thus offers the sole opportunity to describe the foliation planes and fractures intersecting the borehole.

The interpretation of image logs in active fault zones (Pezard and Luthi, 1988; Barton et al., 1995; Brudy and Zoback, 1999; Daniel et al., 2004; Hickman and Zoback, 2004 ; Conin et al., 2014) revealed some of the structural, stress, and permeability conditions at depth, which control the behaviour of fault zones (e.g., Townend et al., 2009; Faulkner et al. 2010). In recent years, drilling near active faults was conducted directly after large earthquakes (Ito and Kiguchi (2005), Nojima Fault, 1995 Kobe earthquake; Hung et al. (2009), Chelungpu Fault, 1999 Chi-chi earthquake; Lin et al. (2013), 2011 Tohoku-Oki earthquake; Nie et al. (2013), 2008 Wenchuan earthquake). Contrary to these drilling projects, the Alpine Fault is late in its interseismic cycle (Berryman et al. 2012), so the DFDP-2B borehole yields information on the conditions 


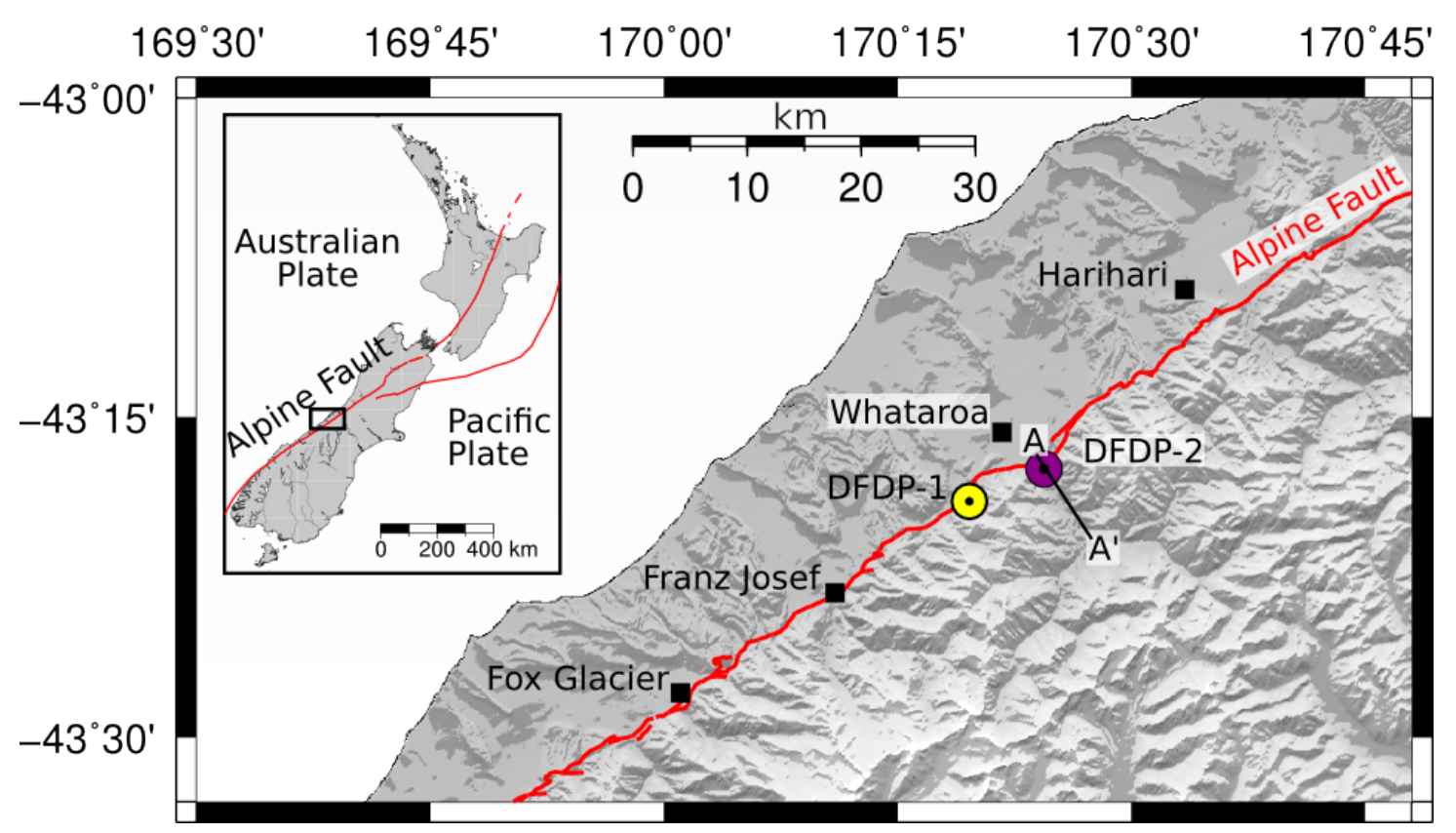

Figure 5.1: Shaded digital elevation model with DFDP-2 and DFDP-1 boreholes, Alpine Fault and nearby townships locations. A-A': cross-section shown in Figure 5.2. The inset shows the map location in New Zealand, and plate boundaries as red lines.

near this major fault before a large earthquake occurs. Characterising the structures in the hangingwall of a major active fault as in DFDP-2B, which represent the cumulative effect of successive deformation events, reveals eventual subsidiary faults (Chester et al., 1993; Lin et al., 2007) and provides a step forwards the characterisation of its style of deformation (Caine et al., 1996, Faulkner et al., 2010).

Schist lithologies constituting the protolith of the Alpine Fault's hangingwall are rarely described using image logs (see Burns, 1987; Barton and Zoback, 2002). Foliated gneiss lithologies have been more commonly studied, but often rely on resistivity image logs, or on drill core descriptions (Paillet and Kapucu, 1989, Barton and Zoback, 2002; Williams and Johnson, 2004, Blake and Davatzes, 2012). It was thus necessary to develop a specific BHTV log analysis method for this work, based on a descriptive classification and integration with outcrop studies.

In this chapter, we study the foliation and structures observed in the hangingwall schist protolith along a continuous sequence where deformation increases as the Alpine Fault is approached, using the high-resolution BHTV logs acquired in the DFDP-2B borehole. First, we present the geological settings of the DFDP-2B borehole. Secondly, we detail the BHTV logs acquisition, processing and analysis methods. Thirdly, we present the results of the analysis of planar features (foliation planes and fractures), and the delineation of acoustic image facies. Finally, we interpret these acoustic image facies and planar features in the context of existing outcrop studies of the Alpine Fault's hangingwall, and discuss the implications on permeability for the fracture system present in the hangingwall of the Alpine Fault. 


\subsection{Geological settings}

The Alpine Fault is a $\sim 850 \mathrm{~km}$-long dextral transpressive fault which forms part of the plate boundary between the Pacific and Australian plates in the South Island of New Zealand (Sibson et al., 1981). The central Southern Alps section of the Alpine Fault has an average strike of $\sim 055^{\circ}$ and dip of $45-60^{\circ}$ SE (see e.g. Norris and Cooper, 2007; Stern et al., 2007) but is segmented in the subsurface into a series of oblique-reverse (SE-dipping) and strike-slip (vertical) sections (Norris and Cooper, 2007). Rapid exhumation along the fault plane brings to the surface rocks that experienced mid-crustal conditions only 1-2 Myr ago (Little et al., 2005, Norris et al., 1990). Ductily deformed schist-derived lithologies (protomylonites, mylonites and ultramylonites) reflect an increasing degree of deformation with proximity to the principal slip zone Norris and Cooper, 2007; Toy et al., 2012). Protomylonites have L-S fabrics, millimeter- to centimetre-spaced foliations of quartz-feldspar and mica \pm amphiboles, and retain early fold structures (Little et al., 2002; Norris and Cooper, 2007; Toy et al., 2012). In outcrop, the mylonitic foliation dips $\sim 25-65^{\circ} \mathrm{SE}$, sub-parallel to the Alpine Fault, and is cross-cut by joints related to the exhumation of the hangingwall (e.g. Holm et al., 1989, Little et al., 2002; Toy et al., 2012; Gillam et al., 2013). Ductily deformed quartzofeldspathic vein segregations are arranged sub-parallel to this foliation.

The two boreholes drilled during the first phase of the DFDP programme (DFDP-1A, $100 \mathrm{~m}$ depth; DFDP-1B, $152 \mathrm{~m}$ depth) sampled fault rocks in the central Alpine Fault at Gaunt Creek (Figure 5.1; Sutherland et al., 2012). Lithological (Toy et al., 2015. Schleicher et al., 2015), structural (Williams et al., 2016), physical (Boulton et al., 2012, Ikari et al., 2014, 2015), and hydraulic (Carpenter et al., 2014) properties were obtained from drill cores, while wireline logging analysis and hydraulic measurements provided details of the structure and petrophysical properties of a geochemically distinct alteration zone (Sutherland et al., 2012; Townend et al., 2013). BHTV log interpretation in DFDP-1B indicates that centimetre-thick-scale fractures with orientations similar to that of the Alpine Fault predominate throughout the logged interval, with a subordinate set of north-dipping fractures (Figure 5.5d; Townend et al., 2013).

The second phase of DFDP ("DFDP-2"), was designed to intersect the Alpine Fault at $\sim 1 \mathrm{~km}$ depth so that topographic effects on stress are diminished. DFDP-2B was drilled in 2014 in the Whataroa Valley to a depth of $893 \mathrm{~m}$ measured along the borehole ( $818 \mathrm{~m}$ vertical depth), but did not reach its target depth due to technical difficulties (Sutherland et al. (2015); all depths reported in this chapter are measured along the borehole; Figures 5.1 and 5.2 . . The borehole deviates steadily with depth from vertical at the ground surface to reach a stable inclination of $\sim 46^{\circ}$ and an azimuth of $\sim 340^{\circ}$. Cuttings and wireline logs, run between the bottom of the casing set in the basement rocks $(264 \mathrm{~m})$ and the bottom depth $(893 \mathrm{~m})$, sampled the hangingwall of 


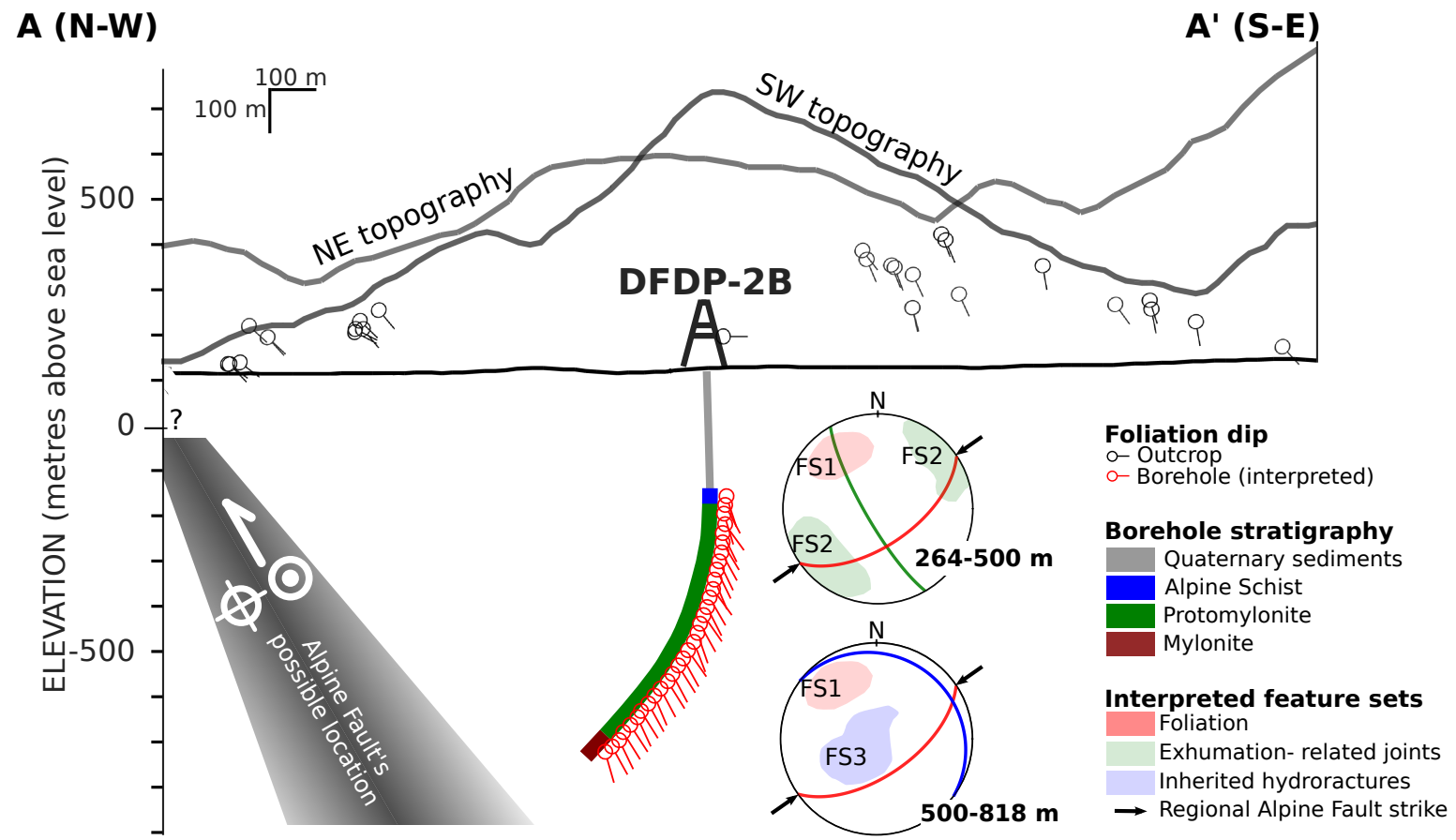

Figure 5.2: Schematic cross-section along the Whataroa Valley (line A-A' in Figure 5.1) of foliation orientations measured in outcrop and interpreted in DFDP-2B; and summary stereonet of the three main feature sets (density contours of poles to planar structures and great circles of mean orientations). The possible locations of the Alpine Fault are shaded in grey, assuming a $50-70^{\circ}$ dip; the location at surface, near-surface geometry and location at depth are not well constrained.

the Alpine Fault in detail (Sutherland et al., 2015). The stratigraphy defined from cuttings analysis comprises Quaternary sedimentary formations from the surface to $260 \mathrm{~m}$, then a succession of schists with an increasing degree of deformation with depth: Alpine Schist to $280 \mathrm{~m}$, protomylonites to $852 \mathrm{~m}$ and mylonites to $893.2 \mathrm{~m}$ (Figure 5.2 . Sutherland et al., 2015). A total of $19.14 \mathrm{~km}$ of wireline logs were acquired during 23 sessions in the $264-889 \mathrm{~m}$ section of DFDP-2B, including $4.8 \mathrm{~km}$ of BHTV logs (Sutherland et al. 2015). From 1 to 13 months after the end of drilling operations (latest measurement to date), a major change in static temperature gradient was measured at $730 \mathrm{~m}$ via a distributed temperature sensing fiber optics installed around the casing (Sutherland et al., 2015, submitted).

\subsection{Borehole Televiewer (BHTV) Log Processing and Analysis Methods}

\subsubsection{BHTV Logging and Processing}

BHTV tools emit and receive ultrasonic pulses using an acoustic transducer located in a $360^{\circ}$ rotating head, to generate two oriented acoustic maps of the inside of the bore- 
hole, one with travel-time and the other with amplitude (Zemanek et al., 1970). The two-ways travel-time of the ultrasonic wave measures the distance between the borehole wall and the tool, and hence estimates the borehole's shape and diameter. The ultrasonic wave amplitude depends on the acoustic impedance of the borehole wall, which depends primarily on the borehole wall roughness, and hence on mineralogy, texture and fracturing (e.g., Davatzes and Hickman, 2010). Log acquisition and processing are described in Appendix 5.A. All BHTV images and interpreted feature orientations presented in this chapter are with reference to geographic north, and borehole deviation has been taken into account during log processing.

An estimation of the borehole diameter ("caliper") is required for the calculation of the orientation of planar features detected in BHTV logs (Zemanek et al., 1970). Discrete increases in borehole diameter are good indicators of individual fractures or fractured zones, while larger-scale variations can indicate larger fractured zones or changes between harder and softer rocks (e.g., Massiot et al., 2015). Reliable caliper measurements are also important for the interpretation of other well logs, in particular the resistivity logs (Remaud, 2015). A three-arm mechanical caliper ran in DFDP-2B did not provide reliable measurements. The method to estimate the borehole diameter from the BHTV travel-time, taking into account the variable speed of sound in the drilling mud, is presented in Appendix 5.B.

\subsubsection{BHTV Log Quality}

The BHTV logs were of variable quality due to mud attenuation, and drilling and logging tool effects (see Appendix 5.C, Lofts and Bourke, 1999). Identifying image artefacts and their causes is a critical step in the analysis of acoustic image facies, as well as of feature orientation and density variations with depth. The detection of planar features is reduced in areas where BHTV image data do not cover the full circumference. This study is based on a compilation of the best quality logs for the 264-888 m interval (Appendix 5.A).

Image quality is ranked within three levels primarily defined by borehole azimuthal coverage: bad, poor and good image quality correspond to $<25 \%, 25-50 \%$ and $>50 \%$ coverage respectively. The presence of artefacts further decreases the quality ranking. Overall, the image quality is good with small intervals of bad quality in the 260$558 \mathrm{~m}$ interval (Figure 5.3). In the 558-598 $\mathrm{m}$ interval, image quality is bad with only small portions of the borehole observable. The 598-893 $\mathrm{m}$ interval is characterised by intercalation of thin $(<1 \mathrm{~m})$ zones of bad to poor quality within otherwise zones of good image quality (Figures 5.3 and 5.8). These thin intervals of poorer quality likely reflect spalling from the borehole wall either due to drilling or the presence of intensely fractured zones. Particular attention will be given to the $476-545 \mathrm{~m}$ interval where the image quality is best. 


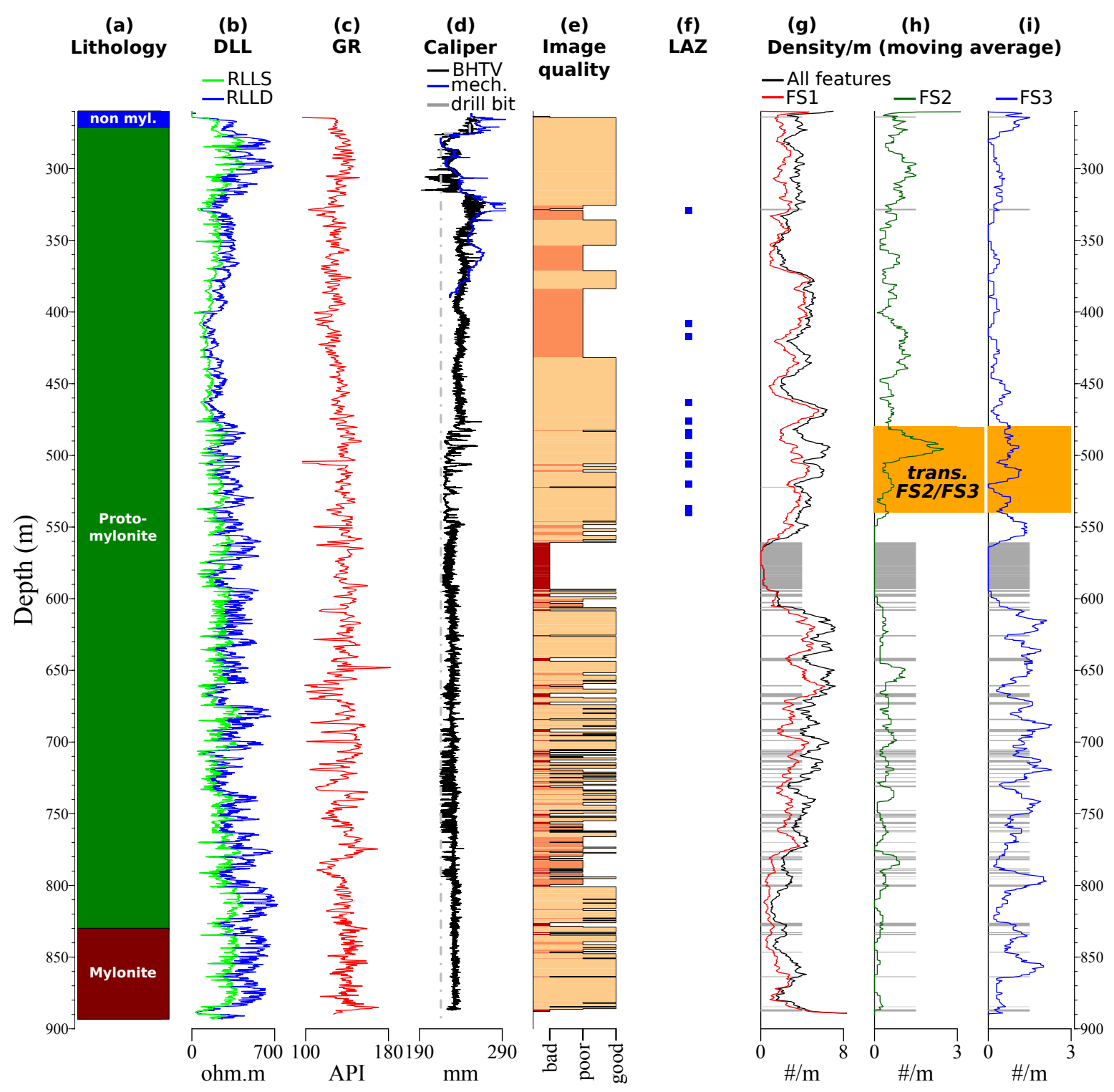

Figure 5.3: Composite of BHTV log interpretation and other wireline logs used in this study. From left to right: a) lithology, b) dual laterolog (shallow resistivity RLLS, deep resistivity RLLD), c) natural gamma-ray, d) caliper: mechanical, computed from BHTV travel-time (Appendix 5.B), and drill-bit-size, e) BHTV log quality, f) occurrence of low acoustic amplitude zones; g-i) planar features density with a $10 \mathrm{~m}$ window moving average: for all features and FS1 features (g), FS2 (h) and FS3 (i). Note the different scale between $\mathrm{g}$ ) and h-i). The 480-540 m transition zone between FS2 and FS3 predominance is highlighted by an orange shaded rectangle. Grey zones in $g$ - $i$ are of bad BHTV log quality. 

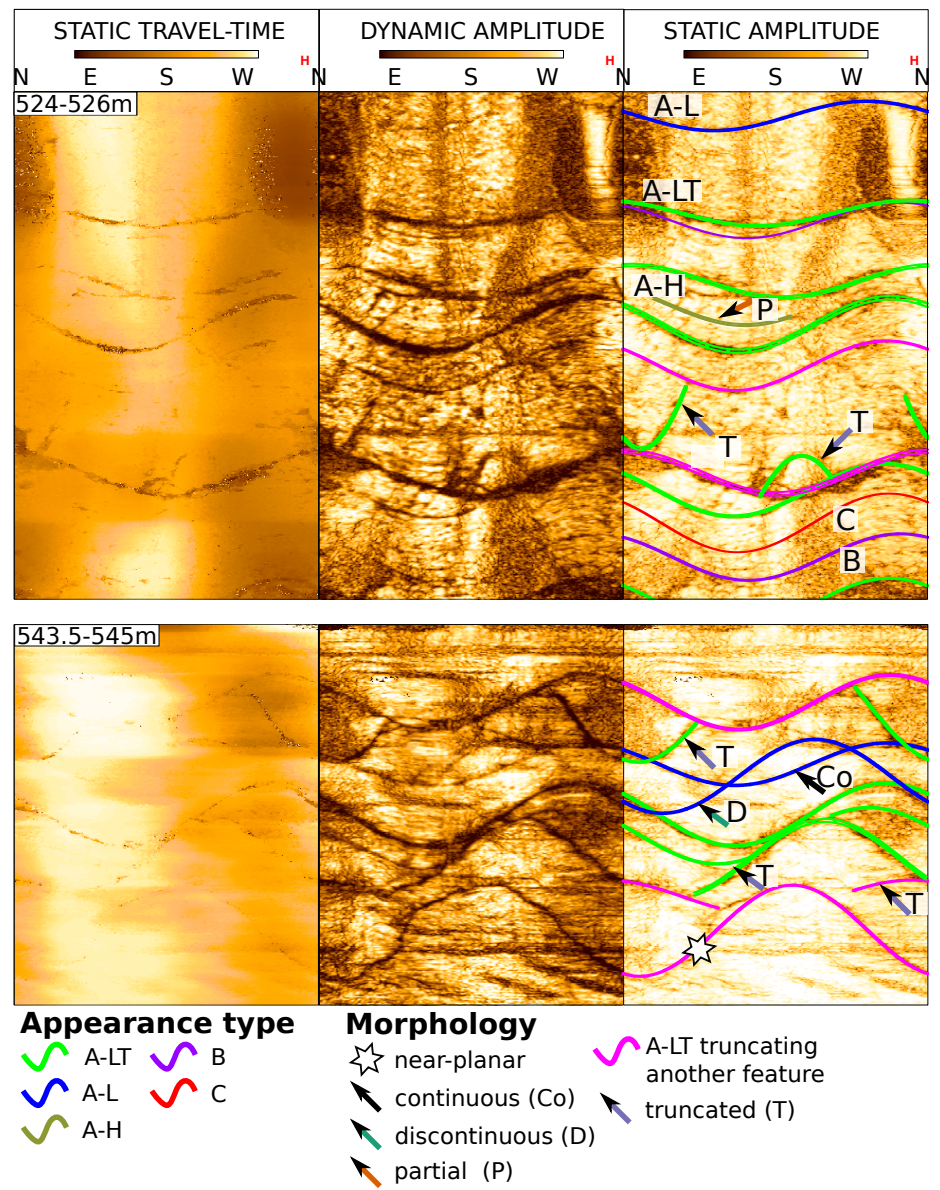

Figure 5.4: Feature morphology and descriptors. A-LT, A-L, A-H, B and C refer to the appearance of features; Co, D, P and T refer to morphological descriptors (see Table 5.1 and 5.2 for more details). Images are displayed unwrapped clockwise and oriented to geographic north.

\subsubsection{Characterisation of Planar Features}

Planar features which have a contrast in acoustic impedance with the surrounding formation appear as a sinusoid on unwrapped BHTV logs (Zemanek et al., 1970). The BHTV log alone cannot differentiate between fractures (used here in a general term, i.e. including faults), veins, and mineral segregations forming part of the foliation (mica and quartz-rich layers). Thus, we use a descriptive classification system of the planar features in terms of contrast, morphology, planarity and thickness (Figure 5.4).

The contrast of acoustic amplitude of planar features with respect to background observed on the amplitude images is classified into three types: A, B and C (Table 5.1. Figure 5.4). Type A features are strongly contrasting. When of low acoustic amplitude, they are labelled A-L, or A-LT in the case where they are also visible on the travel-time image. When of high amplitude, they were never seen on the travel-time image, and labelled A-H. Type B and C are moderately and weakly contrasted, respectively. Type $\mathrm{B}$ and $\mathrm{C}$ features are sampled by a representative sinusoid every $10-30 \mathrm{~cm}$ where they belong to a series of sub-parallel features. 
Table 5.1: Appearance of the planar features.

\begin{tabular}{cccc}
\hline Type & Amplitude contrast & Amplitude value & seen on travel-time image \\
\hline A-LT & High & Low & Yes \\
A-L & High & Low & No \\
A-H & High & High & No \\
B & Moderate* & Low & No \\
C & Low $^{*}$ & Low & No \\
\hline
\end{tabular}

*: Part of a series of sub-parallel, closely spaced $(1-3 \mathrm{~cm})$ sub-planar features.

Table 5.2: Morphology of the planar features.

\begin{tabular}{ll}
\hline Continuity & Definition \\
\hline Continuous (C) & $\begin{array}{l}\text { Full sinusoid observed }>270^{\circ} \text { around the borehole } \\
\text { Discontinuous (D) }\end{array}$ \\
$\begin{array}{l}\text { Sinusoid observed in parts }>270^{\circ} \text { cumulated around the } \\
\text { borehole }\end{array}$ \\
$\begin{array}{l}\text { Partial sinusoid (i.e. observed } 90-270^{\circ} \text { around the borehole) } \\
\text { with at least one end observed }\end{array}$ \\
$\begin{array}{l}\text { Partial sinusoid which stops on another feature } \\
\text { Obscured (O) }\end{array}$ & Partial sinusoid with both ends masked by bad image quality \\
\hline
\end{tabular}

The morphology of each feature is described in terms of its continuity around the borehole in 5 categories (Table 5.2): continuous, discontinuous, partial, truncated and obscured, the latter linked to poor image quality. Planarity is classified in two categories: planar and near-planar, according to how closely they fit a sinusoid. The apparent thickness of each type A feature is measured on the image and converted to the true thickness measured orthogonally to the feature (Barton and Zoback, 1992; Massiot et al., 2015). The thickness of type B and C features is too small to be evaluated. While the smallest observed apparent thickness is $2 \mathrm{~mm}$ (the BHTV log resolution), the conversion from apparent to feature-normal thickness reduces the thickness value, which can thus become lower than the BHTV log resolution.

The analysis of feature orientations is made on a weighted dataset which compensates for the under-sampling of features sub-parallel to the borehole (Terzaghi, 1965 ; Yow, 1987; Massiot et al., 2015). Because of the steadily increase in borehole deviation, the orientation of features sub-parallel to the borehole most affected by the undersampling varies with depth, as presented in Figure 5.5.

No stress-induced features (Zoback et al., 2003; Massiot et al., 2015), were observed in DFDP-2B BHTV logs with confidence.

\subsubsection{Acoustic Image Facies Delineation}

Variations in the gross appearance of BHTV logs can be observed on the statically normalised images. The combination of an acoustic amplitude range (low/high), overall appearance (grainy/smooth), and caliper from the travel-time image, defines acoustic 
image facies. Drilling and logging artefacts account for a number of these variations, and have been clearly isolated.

\subsection{Results}

\subsubsection{Planar Feature Orientation}

The 2242 planar features identified on the BHTV log have three dominant orientations, which we here refer to as feature sets (Figure 5.5. Table 5.3). Feature set 1 (FS1), which contains two thirds of the features, has a constant orientation over the entire logged interval, with a mean dip and dip direction of $60^{\circ} / 145^{\circ}$ and is very clustered (resultant vector length of 0.97; Figure 5.5. Table 5.3, Priest, 1993). This set comprises the foliation and will be discussed in Section 5.6.1. The delineation of the two other feature sets is based primarily on their orientation and coincides with their occurrence in different depth intervals (Section 5.5.2 Figure 5.5). Feature set 2 (FS2) contains NW-SE-striking features steeply dipping $\left(>50^{\circ}\right)$ to both SW and NE directions, with a $89^{\circ} / 047^{\circ}$ mean orientation (Figure 5.5a). Feature set 3 (FS3) contains gently dipping $\left(<50^{\circ}\right)$ features of various dip direction, and a $15^{\circ} / 037^{\circ}$ mean orientation (Figure 5.5b). FS2 and FS3 are less clustered than FS1 (resultant vector lengths of 0.84 and 0.87 , respectively).

\subsubsection{Planar Feature Density}

The feature density is analysed using a moving average of feature density (calculated with a $10 \mathrm{~m}$ window size; Figures $5.3 \mathrm{~g}-\mathrm{i}$ ). The BHTV log quality strongly influences the feature density: zones of bad quality have very few recognised features (see grey shaded zones of bad image quality in Figures $5.3 \mathrm{~s}$-i). While poor log quality may hinder the detection of some features, most type A features are still detectable in these zones. The density analysis is reliable within zones of good image quality but is also made with caution in zones of poor image quality. Type B features are mainly located within

Table 5.3: Number and orientation (dip/dip direction) of planar features for each feature set (FS), characterised by their appearance (Table 5.1), morphology (Table 5.2) and planarity (npl.: near-planar; pl.: planar).

\begin{tabular}{|c|c|c|c|c|c|c|c|c|c|c|c|c|c|c|}
\hline \multirow[t]{2}{*}{ FS } & \multirow{2}{*}{$\begin{array}{l}\text { Ori- } \\
\text { enta- } \\
\text { tion }\end{array}$} & \multicolumn{5}{|c|}{ Appearance } & \multicolumn{5}{|c|}{ Morphology } & \multicolumn{2}{|c|}{ Planarity } & \multirow[t]{2}{*}{ Tot. } \\
\hline & & A-LT & A-L & $\mathrm{A}-\mathrm{H}$ & B & $\mathrm{C}$ & $\mathrm{C}$ & $\mathrm{D}$ & $\mathrm{P}$ & $\mathrm{T}$ & $\mathrm{O}$ & npl. & pl. & \\
\hline FS1 & $60 / 145$ & 972 & 151 & 3 & 98 & 294 & 932 & 185 & 15 & 16 & 370 & 5 & 1513 & 1518 \\
\hline FS2 & $89 / 047$ & 259 & 35 & 0 & 2 & 2 & 97 & 38 & 75 & 36 & 52 & 9 & 289 & 298 \\
\hline FS3 & $15 / 037$ & 332 & 83 & 0 & 7 & 4 & 273 & 91 & 11 & 15 & 36 & 5 & 421 & 426 \\
\hline & Total & 1563 & 269 & 3 & 107 & 300 & 1302 & 314 & 101 & 67 & 458 & 19 & 2223 & 2242 \\
\hline
\end{tabular}



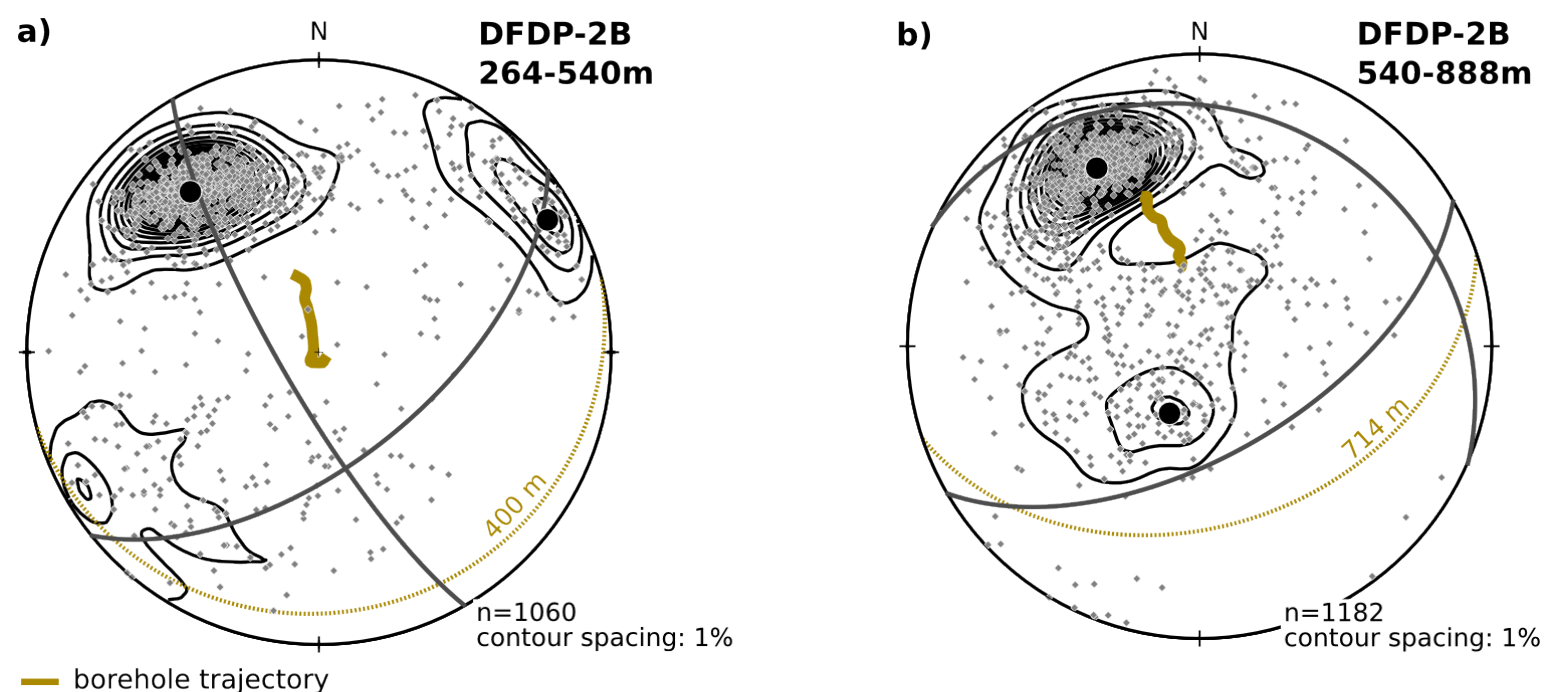

แ"ш! great circle at selected depth containing poles to under-sampled fractures

c)

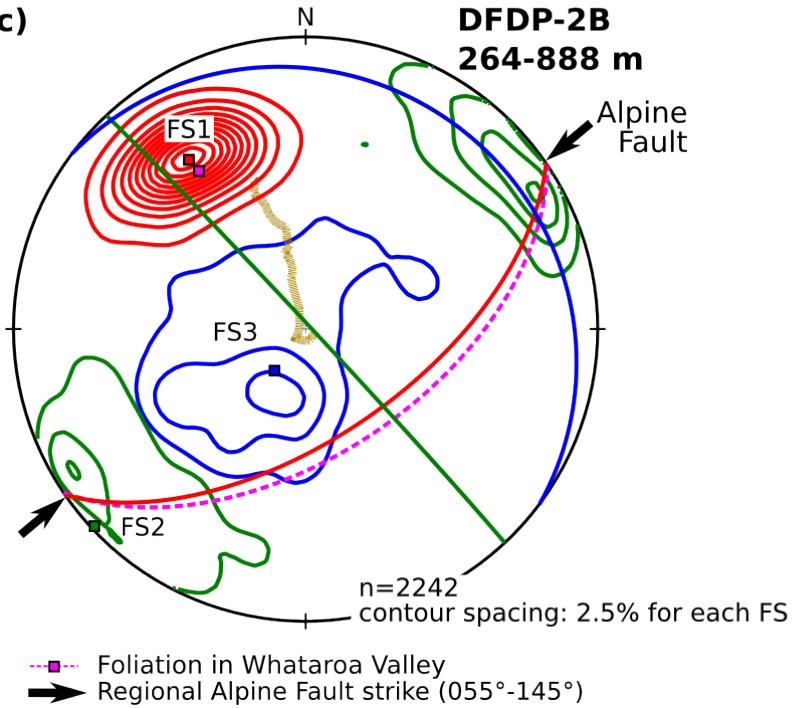

d)

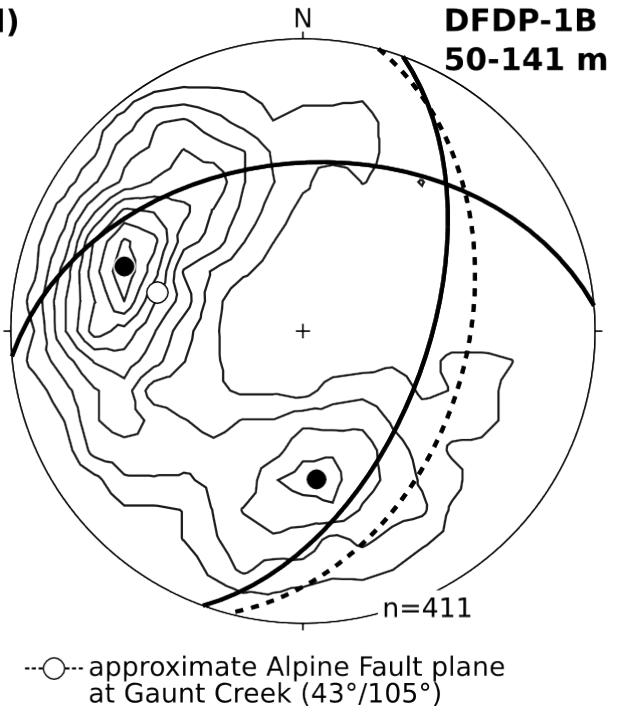

Figure 5.5: Lower-hemisphere, equal-area stereographic representations of features identified in the BHTV logs in DFDP-1B and DFDP-2B boreholes. a) DFDP-2B, 264-540 m interval (number, $\mathrm{N}=1060$ ). b) DFDP-2B, 540-888 m interval $(\mathrm{N}=1182$ ). c) DFDP-2B: Fisher contours and mean orientation of poles to the three interpreted feature sets after being separated $(\mathrm{N}=2242)$; mean foliation orientation in the Whataroa Valley; and regional strike of the Alpine Fault. d) Feature orientation in the DFDP-1B borehole (after Townend et al., 2013) with the approximate Alpine Fault plane orientation at Gaunt Creek as a dashed great circle, white circle representing its pole.

In Figures 5.5a 5.5b, gray symbols are poles to features. Grey lines show Fisher contour intervals at $1 \%$ (Figures 5.5a-5.5b) and 2.5\% (Figure 5.5c) intervals; Kamb contours at intervals of two standard deviations and a significance level of three standard deviations (Figure 5.5d). Large black circles mark the positions of the modal orientations, and solid great circles show the corresponding planes. Yellow dashed great circles on Figures $5.5 a-5.5 b$ represent the poles planes of undersampled features parallel to the borehole at 400 and $714 \mathrm{~m}$, respectively. The orientation of this great circle for each depth is the borehole trajectory (represented as yellow solid line). 
the 490-550 m interval, a zone of good image quality where the acoustic impedance contrast is generally better than in other zones, and are considered to be geologically similar to type $\mathrm{C}$ features.

Overall, the feature density is 3.6 per meter average and up to 7.2 per meter at 620 and $660 \mathrm{~m}$. Figure 5.3 highlights the transition zone between FS2 and FS3 predominance. While FS2 and FS3 features are present throughout the logged interval, FS2 is more frequent than FS3 in the $260-510 \mathrm{~m}$ interval, with a high density peak at 480-500 m, and FS3 is more frequent than FS2 in the 520-888 m interval. The density profiles of the whole dataset and FS1 features alone are very similar, with an average density of 2.4 per meter (Figure $5.3 \mathrm{~g}$ ). FS1 feature density is low $\left(0.3-1.6 \mathrm{~m}^{-1}\right.$ ) at $780-855 \mathrm{~m}$.

\subsubsection{Relationships Between Planar Feature Appearance, Morphology and Orientation}

Type A-LT features have the same distribution of orientations as the overall dataset, which is expected as they represent $70 \%$ of the dataset (Table 5.3). The three type A-H features are of FS1 orientation, and of partial or obscured morphology. Other features of high acoustic amplitude are observed in various locations but are not reported because they span less than $1 / 4^{\text {th }}$ of the borehole perimeter. Nearly all type B and C features are of FS1 orientation.

The majority of features are of continuous morphology (Table 5.3). Partial features are dominantly of FS2 orientation, with a few in the FS1 set. The truncated features are dominantly of FS2 and FS3 orientations and terminate on features of FS1 orientation, although the contrary is also observed. The rare features with near-planar morphologies are found in all three fracture sets.

The detection of near-planar, partial and truncated features requires a good image quality. Poorer image quality below $545 \mathrm{~m}$ likely hinders their detection. It is thus possible that features of these morphologies and FS3 orientation occur more commonly than what is observed on the BHTV log below $545 \mathrm{~m}$.

\subsubsection{Planar Feature Thickness}

The fracture-normal thickness of type A features ranges between $0.5-32 \mathrm{~mm}$ with a mean of $4.2 \mathrm{~mm}$; only $10 \%$ of features have thickness $>7 \mathrm{~mm}$. Type A-LT features are thicker than type A-L's (mean thickness of 4.5 and $2.8 \mathrm{~mm}$, respectively). The three type A-H features are relatively thick $(3.2 ; 6.5$ and $7 \mathrm{~mm})$ compared to other feature types. There is no clear correlation between feature orientation and thickness. The $1 \%$ of features $>15 \mathrm{~mm}$ occur throughout the logged interval, though are more numerous between $476-540 \mathrm{~m}$ and $812-852 \mathrm{~m}$. The thickest feature is located at $520 \mathrm{~m}$, belongs to 
FS1, and is associated with a slight caliper increase and zone of low acoustic amplitude (see Section 5.5.5).

\subsubsection{Low Amplitude Zones and Acoustic Image Facies}

Low acoustic amplitude zones that contain type A features, and where low acoustic amplitude is also observed between the type A features, occur in eleven locations between 260-545 m (Figures 5.3 and 5.6). Low acoustic amplitude zones are 0.5-3 mthick, have brecciated textures, contain numerous type A features, and often coincide with temperature anomalies, low resistivity (deep and shallow), and caliper increases (Figure 5.6). Two configurations of these zones are observed: (1) pervasive low acoustic amplitudes associated with one or more FS1 features, and (2) low acoustic amplitudes confined between two FS1 features. The absence of low acoustic amplitude zones below $545 \mathrm{~m}$ is likely due to the poorer image quality preventing their observation, though some thin $(0.5-3 \mathrm{~m})$ zones of bad image quality may represent low acoustic amplitude zones (Figure 5.8).

The 490-545 m interval is of particularly good image quality with few artefacts. Within this interval, three acoustic image facies (AIF) separated by gradational boundaries are recognised (Figure 5.6):

1. AIF1 has low caliper values $(220-225 \mathrm{~mm}$, drilled with a drill-bit size of $215.9 \mathrm{~mm}$ ), high acoustic amplitude, and smooth borehole walls, as indicated by the uniform travel-time image. AIF1 appears differently when drilled by different drill bits: the amplitude image appears more homogeneous over the intervals drilled with the PDC-type drill-bit than with the tricone IADC-type bit, as seen on overlapping sections of logs (see Appendix 5.C).

2. AIF2 has low caliper values $(220-225 \mathrm{~mm})$ and moderate acoustic amplitude; the amplitude image has a texture that appears thinly foliated.

3. AIF3 has higher caliper values $(>225 \mathrm{~mm})$ and low acoustic amplitude which appears grainy.

\subsection{Discussion}

\subsubsection{Feature Set 1 (FS1): Foliation Planes and Foliation- parallel Fractures}

FS1 features, oriented $60^{\circ} / 145^{\circ}$ on average, are interpreted to be foliation planes, deformed foliation-parallel veins, and/or other foliation-parallel fractures. Surface observations Sibson et al., 1981; Norris and Cooper, 2007; Gillam et al., 2013) and 


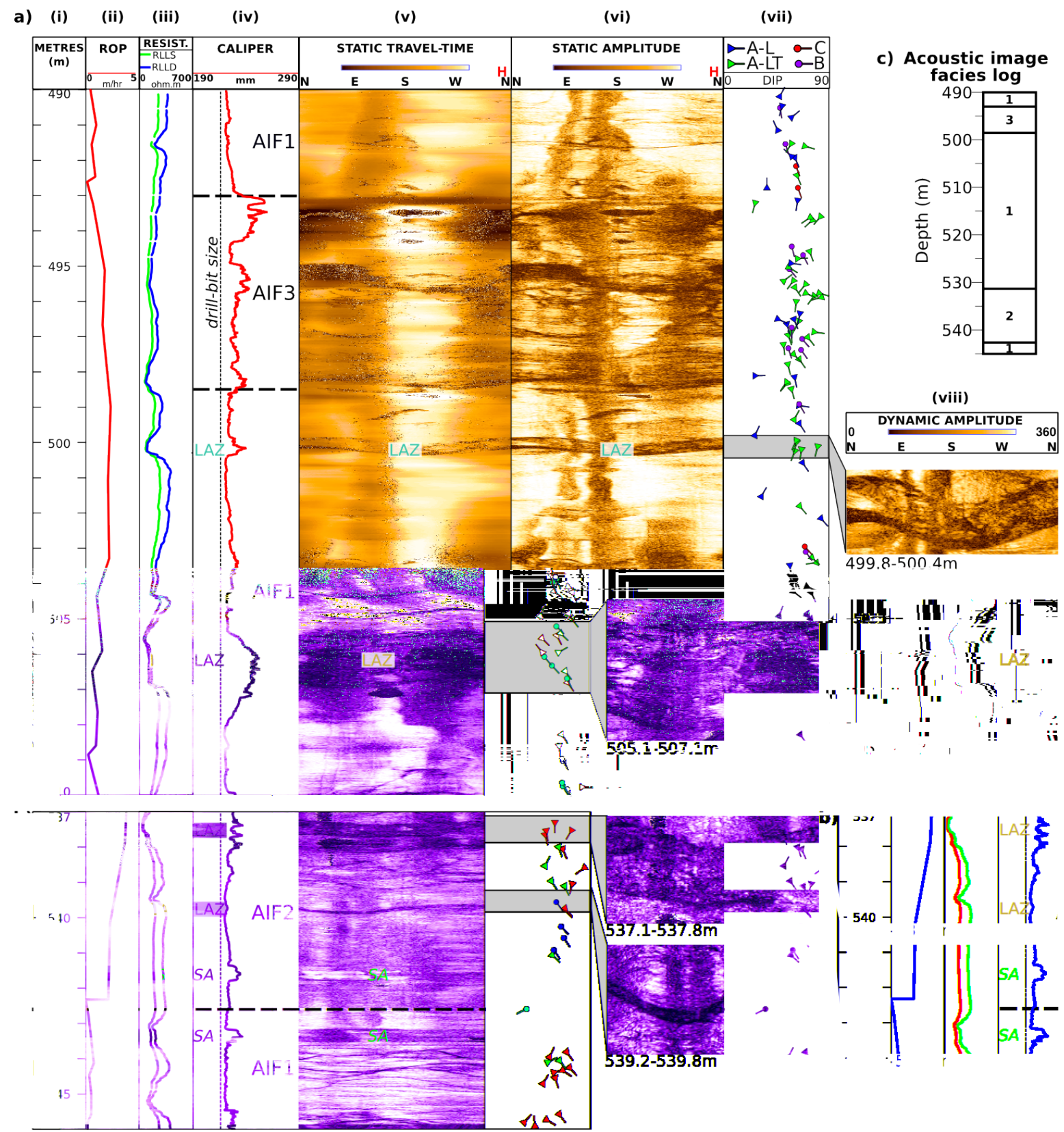

Figure 5.6: Appearance of acoustic image facies (AIF) and low amplitude zones (LAZ). a) $490-510 \mathrm{~m}$. b) $537-546 \mathrm{~m}$. (i) depth; (ii) drilling rate of penetration; (iii) Laterolog (shallow (RLLS) and deep (RLLD)); (iv) caliper calculated from travel-time, and drillbit-size; (v) static travel-time BHTV image; (vi) static amplitude BHTV image; (vii) features orientation (tadpole tail indicate the dip direction; see Table 5.1 for acronyms); (viii) dynamic amplitude BHTV image showing enlargements of LAZ. Note the brecciated texture with centimetric patches of high acoustic amplitude in the low amplitude zones. c) Summary log of the distribution of the three AIF in the 490-545 m interval. "SA": artefact caused by a drill string stabiliser (Appendix 5.C). 
seismic reflection data (Stern et al., 2007; Feenstra et al., 2016) indicate that on average the Alpine fault strikes $\sim 055^{\circ}$ (i.e., a dip direction of $145^{\circ}$ ) and dips $40-60^{\circ} \mathrm{SE}$ at up to $\sim 15 \mathrm{~km}$ depth, before shallowing downwards into a lower crustal detachment at $\sim 35 \mathrm{~km}$ depth. Foliation measured in two well-exposed outcrops of protomylonite and mylonite $\sim 30 \mathrm{~km}$ south of the Whataroa Valley along strike of the Alpine Fault yield average orientations of $63^{\circ} / 143^{\circ}$ at Tatare Stream (Gillam et al., 2013) and $34^{\circ} / 129^{\circ}$ at Stony Creek (Little et al., 2016). In DFDP-2B, FS1 feature orientations are similar to those measured on scarce exposures in the Whataroa valley (Figure 5.2 ; Little et al., 2002 ) and on more extensive ones at Tatare Stream. The progressive deviation of the borehole with depth, to an orientation nearly orthogonal to FS1, is consistent with the effects of drilling through a layered rock mass causing mechanical anisotropy (Misra et al., 2015).

The appearance of the FS1 features supports this geological interpretation. The hangingwall protolith outcropping at Whataroa is strongly foliated (Figure $5.7 \mathrm{a}-\mathrm{b}$ ) with millimetre-to-centimetre-spaced bands of quartz-feldspar and mica \pm amphibole (Little et al. 2002; Toy et al., 2015). Nearly all of type B and C features are also of FS1 feature orientations and have morphological characteristics consistent with foliation planes. Type A features that are part of FS1 are interpreted as foliation-parallel, cmscale mineral segregations (micaceous or quartz-rich layers), and foliation-parallel veins or fractures. The various degrees of acoustic contrast of FS1 features likely relate to changes in mineralogy, or the strength of each layer, which alters the roughness of the borehole wall. The type A-LT features have a high roughness at the borehole wall, and may thus represent slightly excavated mica-rich foliation layers or open fractures. The three type A-H partial features are interpreted to be short quartz veins intercalated with the foliation, as commonly observed in outcrops (Holm et al. 1989 , Little et al., 2002). Although the transition between protomylonite and mylonite is not clear on the BHTV log, the appearance of the foliation on the BHTV image from the lower interval $(>810 \mathrm{~m})$ of DFDP-2B is similar to that in DFDP-1B.

\subsubsection{Foliation Orientation: Implications for the Alpine Fault Geometry}

Structural studies on outcrops reveal that the active Alpine Fault plane bounds a very high-strain mylonite zone characterized by finite shear strains exceeding 120 across most of its width (Norris and Cooper, 2003; Toy et al., 2013). With such high finite strains, the mylonitic foliation becomes parallel to the shear zone boundary (Toy et al. 2013). Previous outcrop studies in the central Southern Alps (including in and near the Whataroa Valley) indicate that the mean attitude of mylonitic foliation within $\sim 1 \mathrm{~km}$ of the Alpine Fault plane is essentially parallel to the Alpine Fault, although in detail such parallelism does not exist everywhere at shorter spatial scales, for example adjacent 

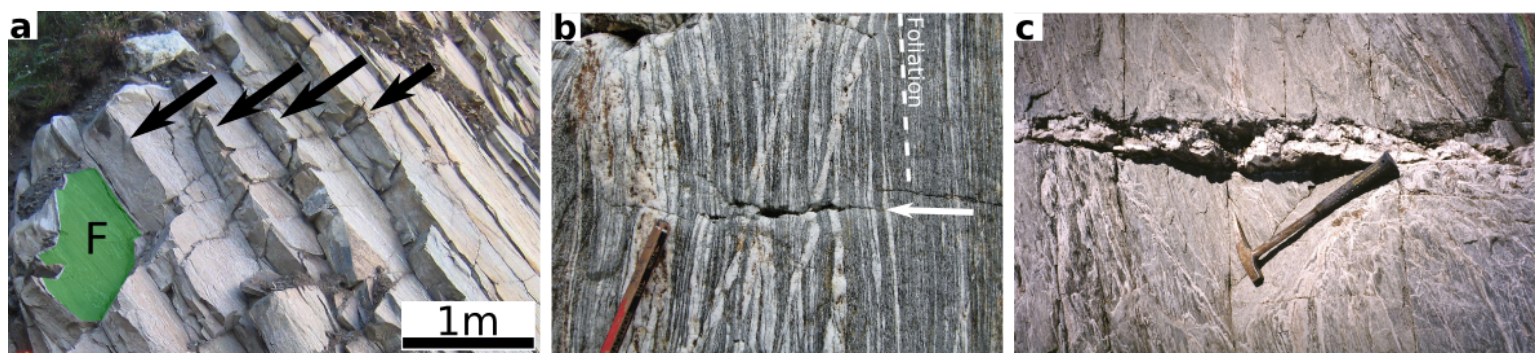

Figure 5.7: Photographs of foliated Alpine Schists, with fractures and veins interpreted to be similar to those in the BHTV log. a) Foliation planes (green shaded plane "F" and other parallel planes), and steeply-dipping foliation-orthogonal joints (arrows) in non-mylonitic Alpine Schist exposed in the Whanganui River quarry, $20 \mathrm{~km}$ north of Whataroa. Such exhumation-related brittle structures are common in outcrops of Alpine Fault hangingwall. b) Open, sub-horizontal extension fracture (white arrow) cutting steeply dipping garnet zone schists at Franz Josef Glacier. c) Sub-horizontal gash vein from the upper Franz Josef Glacier Valley in biotite zone Alpine schists. This open-cavity vein is infilled by quartz+calcite+chlorite+adularia.

to zones of duplexing in the mylonitic zone (Norris and Cooper, 1997). Along-strike of the fault, the mean dip angle of the foliation varies between $\sim 30$ and $60^{\circ}$ within a few kilometres (Little et al., 2016). These changes seem to correlate with alongstrike changes in the near-surface segmentation of the fault, which is characterized by shallower oblique thrust segments; and steeper, linking strike-slip segments, which are inferred to merge at depth into a master fault zone Norris and Cooper, 1995. 1997; Barth et al., 2012). At the $\sim 1-10 \mathrm{~m}$ (outcrop) scale of observation, the local orientation of the mylonitic foliation is more variable as it is commonly complicated by late-stage kinking, thrust duplexing, further brittle deformation, which locally steepens the dip; and gravitational collapse of the scarp, which decreases the dip (e.g., Norris and Cooper, 1997).

Near-surface segmentation and gravitational collapse effects can explain the $\sim 20^{\circ}$ lower dip magnitude and $35^{\circ}$ dip direction difference between the dominant feature orientations observed in borehole DFDP-2B (i.e., FS1) and in DFDP-1B (Figure 5.5). The dominant feature orientation in DFDP-1B is sub-parallel to the approximate orientation of the Alpine Fault plane at Gaunt Creek $\left(43^{\circ} / 105^{\circ}\right.$; Townend et al. 2013$)$, which suggests a possible similar parallelism between foliation and Alpine Fault plane in the Whataroa Valley.

In the DFDP-2B drillhole, we have documented a stable foliation orientation with an average dip of $\sim 60^{\circ}$ toward the SE (dip direction $\sim 145^{\circ}$ ) throughout the protomylonitic to mylonitic hangingwall of the Alpine Fault. This result is significant. About $30 \mathrm{~km}$ SW of the DFDP-2B site, in Tatare Stream, a similarly uniform foliation attitude was observed in protomylonitic to mylonitic outcrops by Gillam et al. (2013). Both Tatare Stream and the DFDP-2B borehole in Whataroa Valley encompass large structural data sets, sample the hangingwall's mylonite zone, and both are located near the SW 
end of an oblique-thrust segment of the Alpine Fault, just east of where the fault transitions westwardly into a strike-slip segment. In the fault segmentation model of Norris and Cooper (1997), such a position is predicted to (uniquely) expose a part of the Alpine Fault that is coplanar with the unsegmented fault plane at depth that lies below the near-surface zone of segmentation. In Tatare Stream, Gillam et al. (2013) interpreted the uniformity of foliation dip as indicating that the mean SE dip of the Alpine Fault at depth in the central Southern Alps (also its ductile shear zone) was $\sim 60^{\circ}$ (see also Little et al., 2005). Based on the assumption that the mean foliation is parallel to the fault plane, a similar interpretation is made here for the DFDP-2B site in the Whataroa Valley on the basis of the BHTV data, though the confirmation of this configuration awaits further drilling.

\subsubsection{FS2 and FS3: Joints Formed During Exhumation, and Inherited Hydrofractures}

FS2 features (sub-vertical and striking NW-SE) are interpreted as joints, probably Neogene, and formed during exhumation. In outcrop, exhumation-related joints are common (Figure 5.7 $\mathrm{a}$; Holm et al. 1989). They often form at $90^{\circ}$ to the foliation, which is the case for FS2 relative to FS1 (Figures 5.5. 5.7 a), and terminate against foliation planes, as observed with truncated FS2 features stopping on FS1 features. In outcrop, these joints are very straight, generally not mineralised, and have lengths of up to tens of metres. This is in agreement with the proportion of partial features which is higher in FS2 than FS1, and suggests that FS1 features are longer than FS2 features Özkaya, 2003).

FS3 features (sub-horizontal) are interpreted as inherited hydrofractures or veins. Such sub-horizontal structures are observed in outcrop in the central Southern Alps (Figure 5.7b-c). Wightman and Little (2007) documented a similar attitude for variably infilled, opening-mode veins in the upper Franz Josef Glacier Valley. These are variably infilled by hydrothermal quartz+calcite+chlorite \pm adularia (see also Craw, 1988, 1997). In Alpine Schists exhumed from mid-crustal depths near Franz Josef Glacier, Holm et al. (1989) documented open, sub-horizontal, extension cracks and also mineralized veins (hydrofractures), that they inferred to record a fluid overpressured stress system associated with a vertical $\sigma_{3}$ direction (minimum principal stress). More recently, Little et al. (2016) used the attitude of pervasive extensional shear bands in the Alpine mylonites to argue that $\sigma_{3}$ was near vertical during late stages of the mylonitic shear deformation - presumably at a site just below the brittle-ductile transition.

The different morphologies of features of FS1 and FS2/FS3 orientations observed in the BHTV log supports the interpretation that the latter structures are joints, i.e.structures propagating in opening mode I susceptible to having terminations on hard, compliant foliation layers. Most truncated features are of FS2/FS3 orientations 
and stop on features of FS1 orientation, while the opposite (features of FS1 orientation stopping either on FS2's or FS3's) is rarely observed (Table 5.3). Situations in which features of the same set terminate on each other also occur, albeit not commonly. While it is possible that the truncated features were formed by separation and slip on the truncating feature (thus indicating a fault), it would have required a large offset $(>0.1 \mathrm{~m})$, which is unlikely for all 67 truncated features. Poor image quality hinders the identification of partial features (true or truncated) in the lower part of the logged interval where FS3 is more common, so the intersection configurations of FS3 cannot be described in as much detail as for FS2.

The occurrence of joints in both FS2 and FS3 orientations is consistent with field observations near the Alpine Fault at Franz Josef Glacier (Holm et al., 1989). In addition, extensional shear bands studies (Little et al., 2016) suggest that a low stress ratio parameter (where $\sigma_{2} \simeq \sigma_{3}$; with $\sigma_{2}$ the intermediate principal stress) allows the switching between $\sigma_{2}$ and $\sigma_{3}$ between the ductile and brittle parts of the crust; this may occur due to post-seismic transient stress rotations, or to stress perturbations close to active faults. The $\sigma_{2}$ and $\sigma_{3}$ directions (either sub-vertical, or sub-horizontal and trending NNE-SSW) obtained by Little et al. (2016) are consistent with the formation of joints orthogonal to $\sigma_{3}$ in FS2 and FS3 orientations.

The depth-dependant change in the proportions of FS2 and FS3 joints that takes place at 480-540 m (Figure 5.3), with FS2 joints more common above this interval and FS3 joints more common below, is unlikely to be an artefact. Indeed, the orientation of FS1 features is constant throughout the logged interval, ruling out a significant error of the caliper log (and hence in the calculation of feature orientations); both FS2 and FS3 are present throughout the logged interval; and the change of proportion of FS2 and FS3 occurs within a zone of moderate to good image quality. The top of this transition zone at 470-480 $\mathrm{m}$ is also conspicuous in other wireline logs (Sutherland et al. 2015 ). Resistivity values are lower, the full-waveform sonic log shows a lower amplitude, and the gamma-ray values are lower between 330-475 $\mathrm{m}$ than between 475-888 $\mathrm{m}$ (Figure 5.3). These log variations reflect lithological or in-situ fluid property changes Rider, 1996, Li et al., 2014a). The cause for the change of proportion between FS2 and FS3 at $\sim 500 \mathrm{~m}$ is not clear; it may relate to local changes of in-situ stresses or pore pressure at depth which would have favoured the formation of hydrofractures in certain zones. Alternatively, joints of FS2 orientation may have a higher acoustic impedance contrast with the background formation in the shallow parts of the borehole, thus favouring their identification, while the converse may happen to FS3. However, if this were the case, a more gradual change of proportion would be expected, and would not occur at the same depth as the other wireline log changes. Future research dedicated to the examination of the relationships between exhumation-related joints and hydrofractures in outcrops may help to explain these observations.

BHTV log interpretation in DFDP-1B borehole reveals the presence of a secondary 
feature set dipping moderately north, sub-perpendicular to the dominant orientation that we suggest represents the foliation (Figure 5.5d; Townend et al., 2013). This configuration is similar to the FS2 joints striking sub-perpendicular to FS1 (foliation) observed in the DFDP-2B borehole. A systematic comparison of BHTV logs and cores in well DFDP-1B, or a future borehole, may provide further information regarding the nature (mineralisation, opening, generation) of FS2 and FS3 features in DFDP-2B.

\subsubsection{Possible Subsidiary Fault Zones in the Alpine Fault Hang- ingwall}

We interpret the low-amplitude zones described in Section 5.5.5 to be damage zones associated with subsidiary faults of the Alpine Fault. Similar patterns have been observed on BHTV logs in DFDP-1B, and are correlated to cataclasite zones in cores (Williams et al. 2015). Cataclastic shears and gouge-filled zones occur throughout the $1 \mathrm{~km}$-wide fault zone (Norris and Cooper, 2007). Daniel et al. (2004) reported analogous bed-contained brecciated layers inferred from resistivity and BHTV image logs, and correlated to cores in the AIG10 borehole, Gulf of Corinth. In DFDP-2B, pervasive hydrothermal clay alteration from fluids circulating along permeable type A fractures may cause the diffuse low acoustic amplitudes, as observed in the Amethyst tunnel near Hari Hari, $15 \mathrm{~km}$ north of DFDP-2B along-strike the Alpine Fault Cox and Sutherland, 2007) and at the Soultz geothermal field (France; Genter and Traineau, 1996).

Pelitic- or mica-rich zones could also appear as diffuse low acoustic amplitudes, but the presence of type A features, the limited depth interval within which they occur, and the brecciated appearance, favours the interpretation of these zones as brecciated fault zones. The low acoustic amplitude zones contained between FS1 features may represent foliation-parallel cataclasite zones along which fluid circulation is contained between two specific fracture planes, while those not contained between specific features may result from the percolation of fluids from one or several fractures. The inference of subsidiary faulting occurring in the hangingwall is consistent with subsidiary faults observed on outcrop to the south-east of the main Alpine Fault plane Hanson et al. 1990; Cox and Sutherland, 2007; Norris and Cooper, 2007) and causing earthquakes Boese et al., 2012; Bourguignon et al., 2015).

No significant feature could be identified on the BHTV log at $730 \mathrm{~m}$ where a sharp decrease of static temperature gradient suggests a change of hydrological regime, associated with drilling mud losses while drilling (Figure 5.8, Sutherland et al., 2015, submitted). Measurements made following drilling of the DFDP-1A and DFDP-1B boreholes demonstrated that a few centimetres of clay gouge is sufficient to create an impermeable barrier to flow, with a difference of three orders of magnitude in permeability measured either side of the principal slip zone of the Alpine Fault Sutherland 


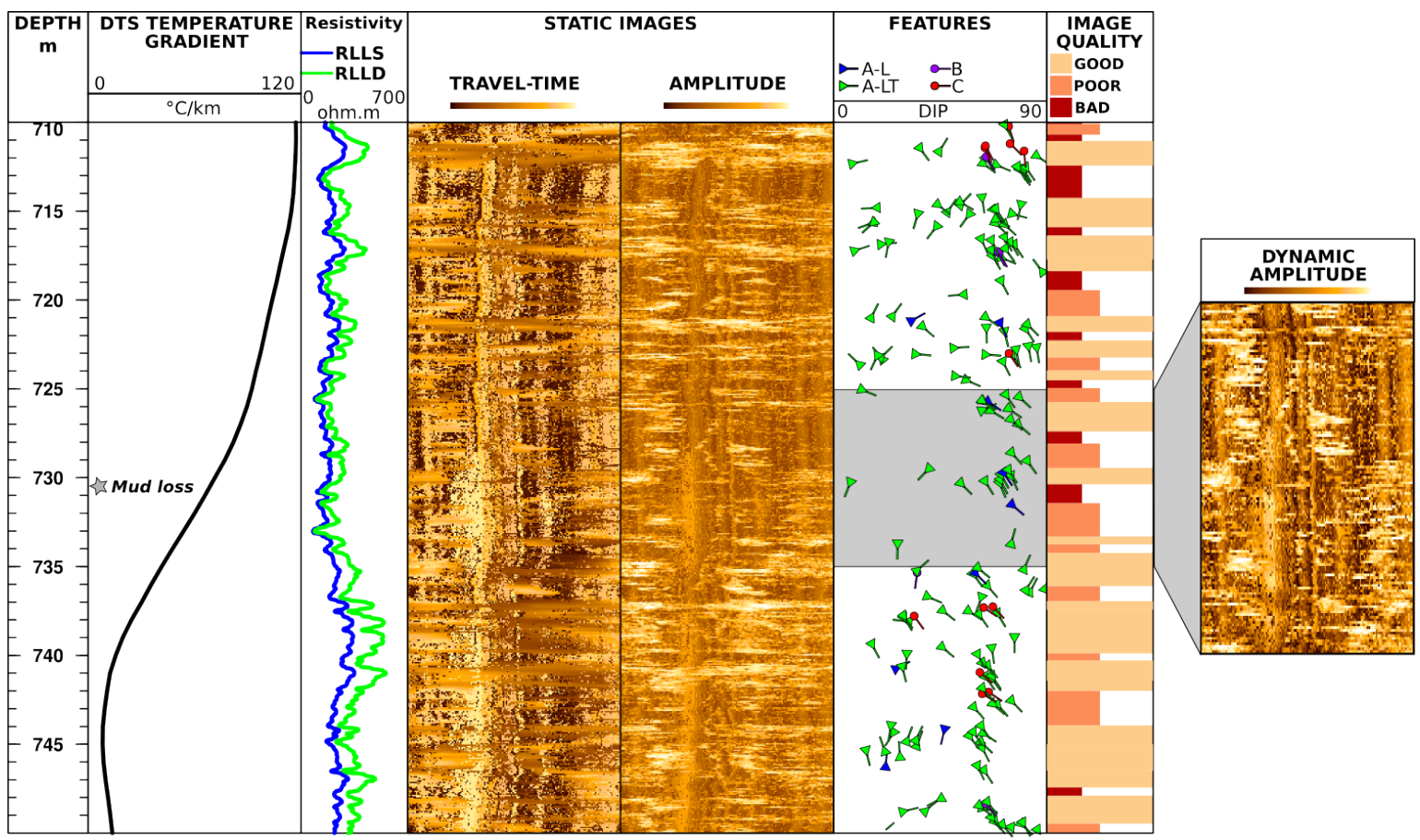

Figure 5.8: BHTV log of the 710-750 m interval surrounding a major static temperature gradient change and mud loss while drilling, showing numerous thin intervals of bad and poor image quality within intervals of good image quality. From left to right: depth, temperature gradient from distributed temperature sensing cable, resistivity, static travel-time image, static amplitude image, tadpoles of interpreted features, BHTV log quality, dynamic amplitude image enlargement of the 725-735 m interval surrounding the sharp decrease in temperature gradient and mud loss zone.

et al., 2012). Similar observations have been made at other fault drilling projects (e.g., Li et al. 2013). It is possible that a centimetre-thick, clay gouge layer occurs at $\sim 730 \mathrm{~m}$ in DFDP-2B but is not resolved on the BHTV logs. Zones of poor BHTV log quality at $\sim 728$ and $732 \mathrm{~m}$ may represent the damage zone of a low-permeability fault responsible for this major temperature gradient change (Figure 5.8). Similarly, the lack of low acoustic amplitude zones below $558 \mathrm{~m}$ is likely caused by the overall poorer BHTV $\log$ quality rather than their absence in the rock.

\subsubsection{Interpretation of the Acoustic Image Facies}

Hard formations, and some minerals including quartz or calcite, tend to have lower caliper and higher acoustic amplitude than softer formations or clay and micas minerals, due to their higher acoustic impedance and lower roughness at the borehole wall (Zemanek et al., 1970; Massiot et al., 2015). In the 490-545 m interval, some interpretation of the three acoustic image facies delineated in Section 5.5.5 is possible.

The acoustic image facies AIF1 and AIF2 are inferred to be hard, quartz-rich formations as indicated by their high acoustic amplitude and low caliper, with AIF2 appearing more thinly foliated or grainy than AIF1. This inference is also supported 
by the presence of hard layers which wore out the drill bits and slowed down the drilling progress in the 519-524 $\mathrm{m}$ interval and at $546.8 \mathrm{~m}$, both within AIF1. The rate of penetration is high between $527-543 \mathrm{~m}$, similar to the interval where AIF2 occurs, suggesting that there are fewer hard quartz veins (Figure 5.3). Thus, the lithology corresponding to AIF1 may be harder and contain more, thicker, or more continuous quartz veins than AIF2. The gamma-ray log does not provide meaningful information at this depth.

A very low gamma-ray and high deep resistivity anomaly is observed on multiple logging runs at 504-505.5 m, which could indicate a low-mica or low-clay zone, and conversely a high-quartz zone. This interval has a high acoustic amplitude and low caliper, similar to the characteristic of AIF1.

AIF3 (493-498.5 m) is a softer formation with higher borehole wall roughness than AIF1/AIF2, and may contain more mica and/or clay minerals as indicated by the lower acoustic amplitude. The AIF3 interval is associated with a low resistivity which may be caused by a clay- or mica-rich formation, and/or by a higher fluid content. The latter is consistent with the high density of type A features observed in AIF3 which may be permeable fractures.

While we were able to describe acoustic image facies in details only on a $55 \mathrm{~m}$-long interval of the logged interval, observations highlight local variations in quartz:mica content or in alteration which cannot be identified from cuttings alone, sampled only every $2 \mathrm{~m}$ and subject to mixing within the borehole. The possible variations of mineralogy, rock hardness or layering style that we have interpreted here may cause 1-10 m-scale variations in formation strength and anisotropy along the borehole. These variations likely occur in other locations in the hangingwall of the Alpine Fault and may also locally impact the fracture distribution.

\subsubsection{Perspectives: Implications for Permeability}

An exhaustive analysis of the relationship between fracture orientation and permeability is beyond the scope of this study, but inferences can be made on potential fluid flow directions in the hangingwall of the Alpine Fault.

Among the various features, type A-LT features are the most likely to be permeable as they locally increase roughness at the borehole wall, though it is not known how far these fractures propagate into the formation. The zones of low acoustic amplitude interpreted to be subsidiary faults with damage zones (Section 5.6.4) are good candidates for contributing to permeability in the hangingwall, although they may be partially clogged with clays. The metre-scale zones of poor BHTV log quality between 545-888 m, which may represent densely fractured zones composed from structures of all feature sets (FS1-3) and induce spalling from the borehole wall, may also be permeable. 


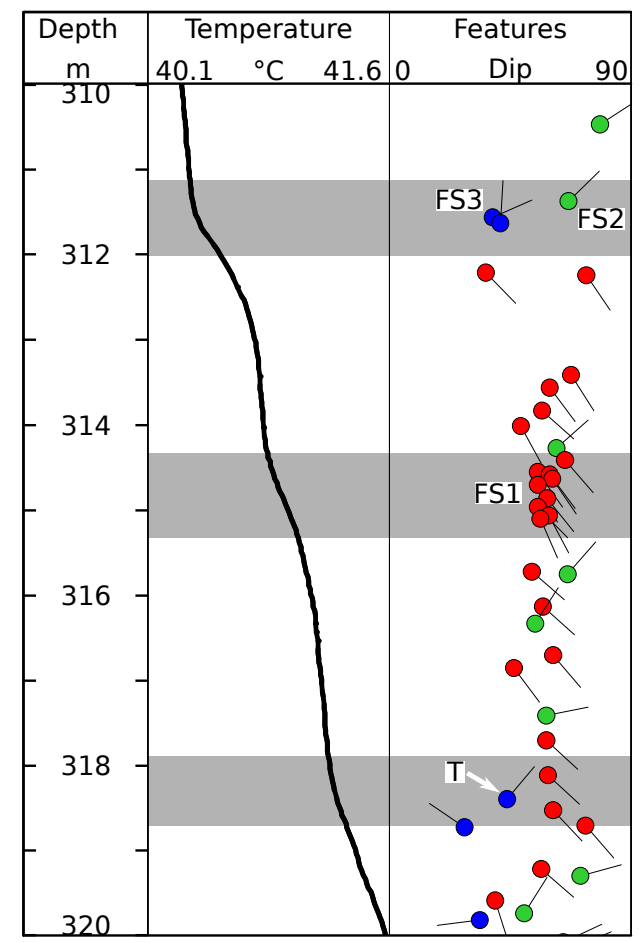

Figure 5.9: Examples of permeable fractures of each orientation set, and at the intersection between a truncated (T) FS3 and a continuous FS1 fracture, identified by temperature changes (shaded areas). FS1, FS2 and FS3 features are represented as red, green and blue tadpoles, respectively.

At least some of the foliation-parallel fractures (FS1), exhumation-related joints (FS2) with minimal mineralisation, and hydrofractures (FS3), are not fully sealed and likely permeable, as seen by temperature gradient changes (Figure 5.9). Foliationparallel fractures alone would not constitute a well-connected fracture system, but their intersection with fractures of FS2 and FS3 orientations, at high angles to the foliation, would provide this connectivity. The truncated fractures of FS2/FS3 orientations stopping on foliation planes are an example of such connections and are in places associated with temperature anomalies (Figure 5.9). FS2 joints, dominant above $500 \mathrm{~m}$, may connect foliation planes vertically up to the quaternary sediments, while FS3 joints, dominant below $500 \mathrm{~m}$, may favour lateral connectivity. The change of proportion of FS2/FS3 at 480-550 m thus likely impacts fluid circulation in the hangingwall of the Alpine Fault in the Whataroa Valley.

Future work dedicated to the integrated interpretation of the BHTV log, including planar feature thickness and density variation with depth, together with the other wireline logs and mud parameters, will help delineate the controls on thermal and fluid flow properties near the DFDP-2B borehole. This may in turn further improve fluid flow and stress modelling near the Alpine Fault Townend et al., 2013, Sutherland et al. submitted). 


\subsection{Conclusion}

The interpretation of BHTV logs acquired in the DFDP-2B borehole yields a detailed description of the foliation and fractures in the hangingwall of the central Alpine Fault. The constant foliation orientation of $60^{\circ} / 145^{\circ}$ observed in the borehole is similar to outcrop measurements in the Whataroa and nearby Tatare valleys, and suggests that at this location, the foliation orientation reflects the orientation of the deeper Alpine Fault plane. Outcrop observations support the interpretation that auxiliary FS2 (sub-vertical striking NW-SE, and mainly above $\sim 500 \mathrm{~m}$ ) and FS3 (sub-horizontal, and mainly below $\sim 500 \mathrm{~m}$ ) fracture sets represent exhumation joints and inherited hydrofractures. The orientation of these joints is consistent with inferred paleo-stress orientations, and magnitudes $\sigma_{2} \simeq \sigma_{3}$. The presence of three acoustic image facies over a $50 \mathrm{~m}$-long interval highlights local variability in lithology and/or alteration. Together with a series of brecciated altered subsidiary faults, foliation-parallel fractures and the two auxiliary fracture sets define the fracture network controlling fluid flow within the Alpine Fault's hangingwall in the Whataroa Valley.

\section{Acknowledgments}

The skills demonstrated by J. Paris, G. Henry and O. Nitsch during logging operations are very valued. We are grateful to M. Lawrence, A. Griffin and W. Kissling for comments on the BHTV analysis and interpretation during and after drilling, A. Pyne for his drilling expertise and S. Keys for his field measurements. The DFDP project is funded by: the International Continental Scientific Drilling Program, (ICDP); the Royal Society of New Zealand Marsden Fund; GNS Science; Victoria University of Wellington; University of Otago; and governments of NZ (MBIE), UK (NERC), and USA (NSF). 


\section{Appendix}

\section{A BHTV Log Acquisition and Processing}

Two BHTV tools developed by Advanced Logic Technology were used to accommodate the high temperatures encountered in DFDP-2B (Sutherland et al., 2015): the ABI40 in the $260-545.86 \mathrm{~m}$ interval and the ABI43 in the $545.86-888 \mathrm{~m}$ interval, able to operate at a temperature up to $70^{\circ} \mathrm{C}$ and $125^{\circ} \mathrm{C}$, respectively (Table 5.A.1). Both tools emit pulses at $1.2 \mathrm{MHz}$. Logs recorded 288 samples per revolution with $2 \mathrm{~mm}$ depth increments, at logging speeds of $0.5-1.2 \mathrm{~m} / \mathrm{min}$. The resulting image pixel size is $\sim 4 \times 2 \mathrm{~mm}$ in the sections drilled with a $241.3 \mathrm{~mm}$ and $215.9 \mathrm{~mm}$ diameter drill bits (264-274.9 and 274.9-893 m intervals, respectively). The ultrasonic wave travel-time and amplitude are converted into images oriented with respect to magnetic north and vertical using an inbuilt three-components accelerometer and magnetometer. These sensors also allow to monitor borehole deviation and azimuth. The dataset is corrected for both the $23.15^{\circ} \mathrm{E}$ magnetic declination and the borehole deviation.

BHTV logs acquired with the ABI40 tool were depth-matched with other wireline logs using the gamma-ray sensors stacked on each logging tool suites Sutherland et al. 2015). The ABI43 tool was not stackable with the available gamma-ray tool, and hence the deepest logs acquired with this tool were adjusted by matching successive overlapping sections.

The raw BHTV logs and acquisition curves were exported from WellCAD and processed in Recall ${ }^{T M}$ 5.4. Two classic image normalisations were used (Rider, 1996): (1) static, which normalises travel-time and amplitude values over the whole log and highlights large-scale variations ( $>1 \mathrm{~m}$ ), and (2) dynamic, which normalises the images over a moving window of given width $(0.5 \mathrm{~m}$ depth in our case) and highlights local variations.

\section{B Calculations of Borehole Diameter (Caliper)}

The first step of the caliper calculation from the BHTV travel-time consists of evaluating the borehole's diameter in the time domain for each depth increment. For each depth increment, a circle was fitted to BHTV travel-time logs acquired with the ABI40 tool. Logs acquired with the ABI43 (546-888 m) had less signal returned, so the circle 


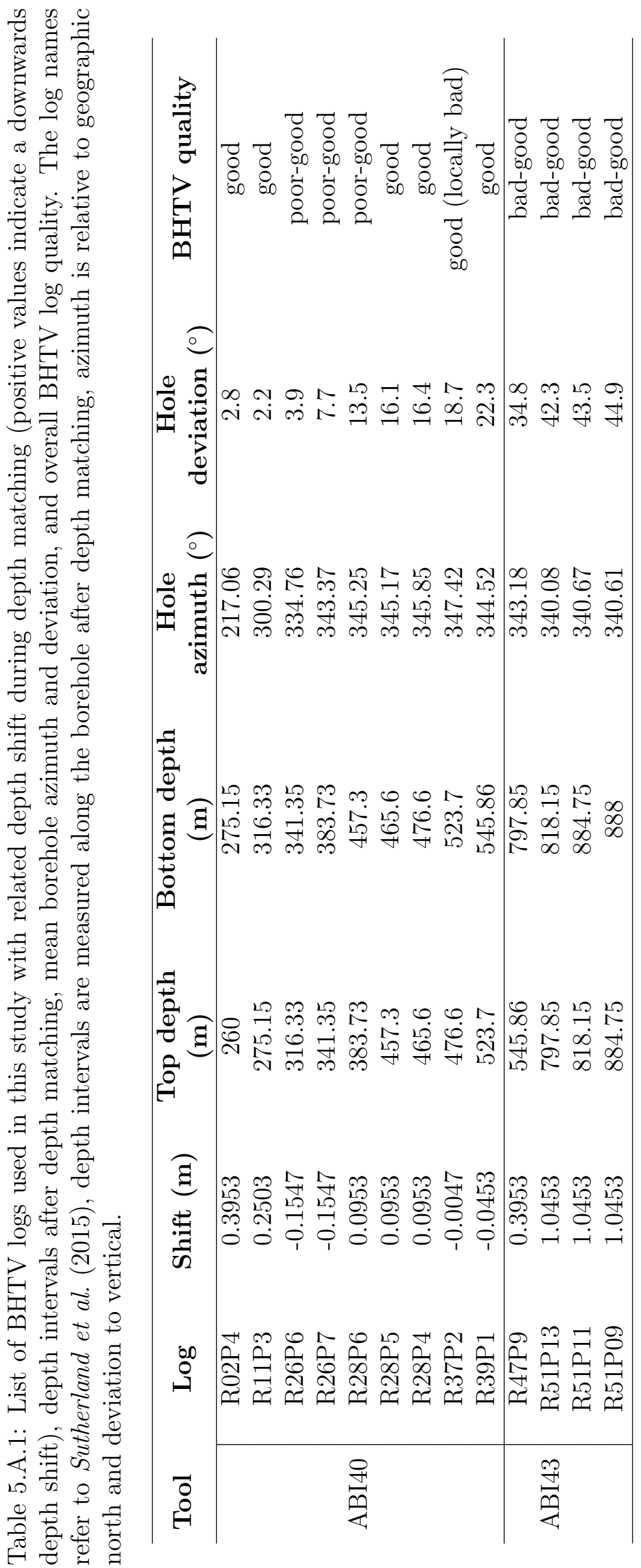




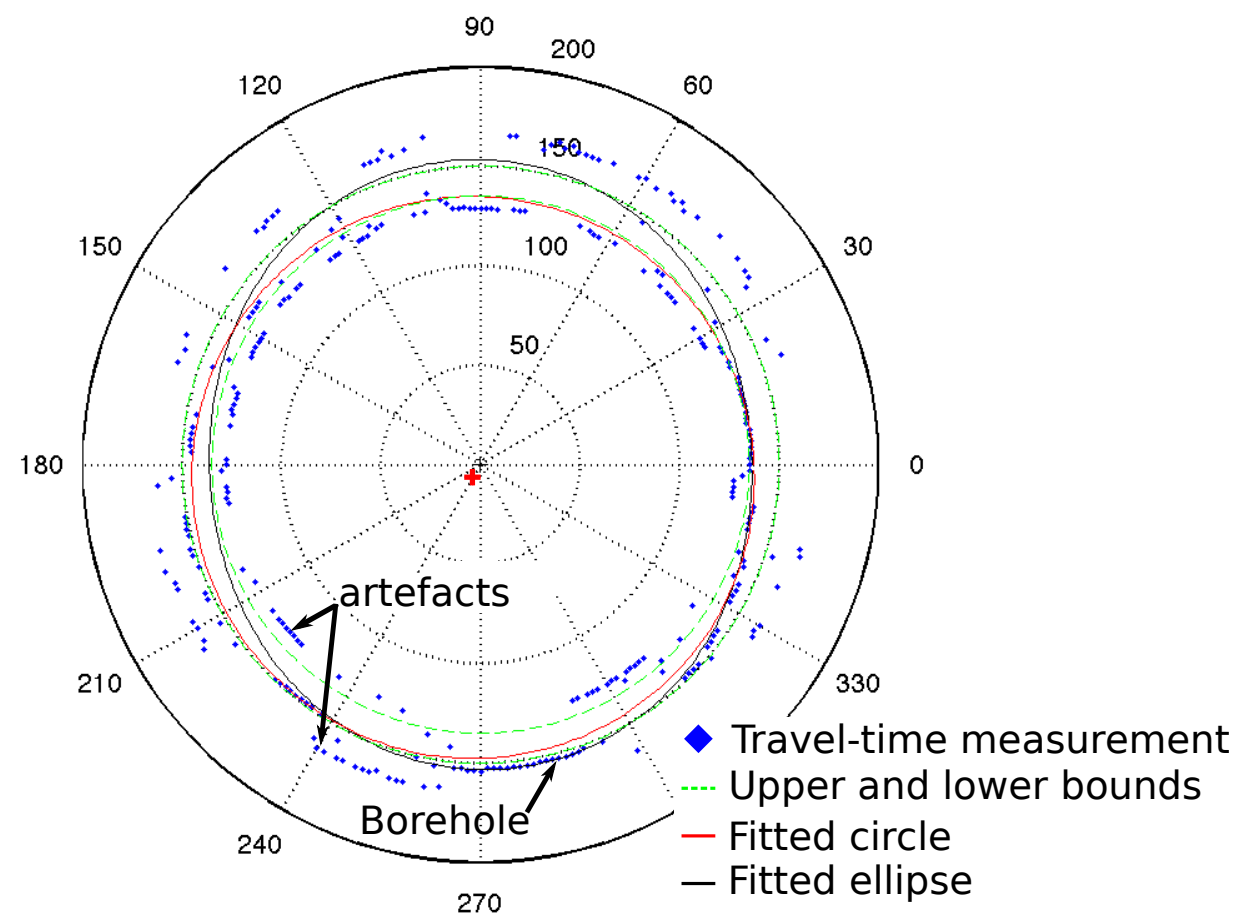

Figure 5.B.1: Example of caliper calculation from BHTV log, with the fitted circle, travel-time measurements around the borehole, and upper and lower bounds of the window time (136 and $157 \mu \mathrm{s}$ in this case). Points within the inner green circle or outside the outer green circle were arbitrarily assigned to the window time bounds during logging and not used for fitting the circle. Circle fitting is made on the raw measurement in micro-seconds (radial coordinates), the circular orientation reference is arbitrary. The fitted circle centre (red cross) indicates here a slight decentralisation.

was fitted on travel-time dataset of five successive measurements (i.e. every centimetre) to increase signal:noise ratio, based on the assumption that the caliper is unlikely to vary significantly over $1 \mathrm{~cm}$ (Figure 5.B.1). To ensure the circle fitted the formation signal and not the internal tool reflections, we imposed minimum and maximum diameters corresponding to the limits of the window time. A fit was deemed good when at least $75 \%$ of the points satisfied this limit. This method limits false detection of tool noise rather than echoes back from the borehole wall. The main limitation of this procedure, however, is its inability to capture diameters larger or smaller than the window time.

The second step of the caliper calculation consists of converting the diameter from time into distance. The speed of sound in the mud was evaluated using thermodynamic equations (Wagner and Kretzschmar, 2007) taking into account temperatures and pressures recorded at each logging depth. As there were no temperature sensors on the ABI tools, temperature logs acquired just prior to each BHTV logging run were used as proxies. Additionally, a coefficient related to the mud density measured at the outflow from the borehole was made to take into account changes in mud properties. The resulting caliper measurements were validated in the casing, in sections of the borehole with high signal to noise return, and with a three-arm mechanical caliper log 
acquired in the upper part of the hole (264-395 m; Figure 5.3). Unfortunately, the mechanical caliper tool was not reliable in deeper sections of the borehole Sutherland et al., 2015).

The quality of the caliper log calculated from the BHTV travel-time relates directly to the BHTV quality: moderate between 260-476 m, very good between 476-545.86 m, variable between 545.86-558 m, bad between 558-598 m and variable between 598$888 \mathrm{~m}$. A bad caliper quality indicates that the caliper may not represent the actual borehole shape. In intervals of bad caliper quality, the caliper log is $\sim 10 \mathrm{~mm}$ bigger than the drill bit size due to the upper limit allowed on the borehole diameter. While this estimation likely underestimates borehole enlargements where return signal is poor, it remains a better proxy for borehole diameter than a constant bit size that would lead to an overestimation of structural dips in enlarged intervals.

\section{C Impact of Drilling and Logging on BHTV Log Quality}

The quality of BHTV logs in the DFDP-2B borehole is predominantly affected by the use of viscous drilling mud (water-based with added bentonite) which attenuates the acoustic signal. In parts of the borehole, the formation signal became lower than that of internal tool reflections and their multiples; the tool may then record the signal from the artefact instead of that from the formation. The ABI tools automatically select the highest amplitude signal returned to the tool within a manually selected time window. To limit false detection of the tool's internal reflections, the time window was adjusted manually in-between conspicuous tool reflections where these reflections had travel-times sufficiently separated from that of formation. This risk of false detection of the tool's internal reflections was amplified by (1) the high temperature gradient along the borehole which modifies the speed of the ultrasonic signal and shifts the arrival time of the internal reflection artefacts while logging; and (2) the eccentralisation in this deviated borehole, and successive reaming of the borehole, which causes the formation signal to arrive at different times around the borehole. These additional issues commonly resulted in parts of the borehole not being imaged. Finally, steel shards stuck in the borehole wall during drilling and fishing operations perturbed magnetic measurements $(+12 \mu \mathrm{T})$; no features were picked in this $30 \mathrm{~cm}$-long interval at $352 \mathrm{~m}$ because the image orientation is unreliable.

The type of drill bit has a strong impact on the appearance of the BHTV logs. The polycrystalline diamond composite (PDC) and tricone IADC 447 bits used for bottomhole-assembly (BHA) 1 to 5 (Sutherland et al., 2015) produced the best images, noting that other parameters related to log acquisition also affect image quality. The tricone IADC tends to increase the apparent aperture of the planar features, resulting in a 


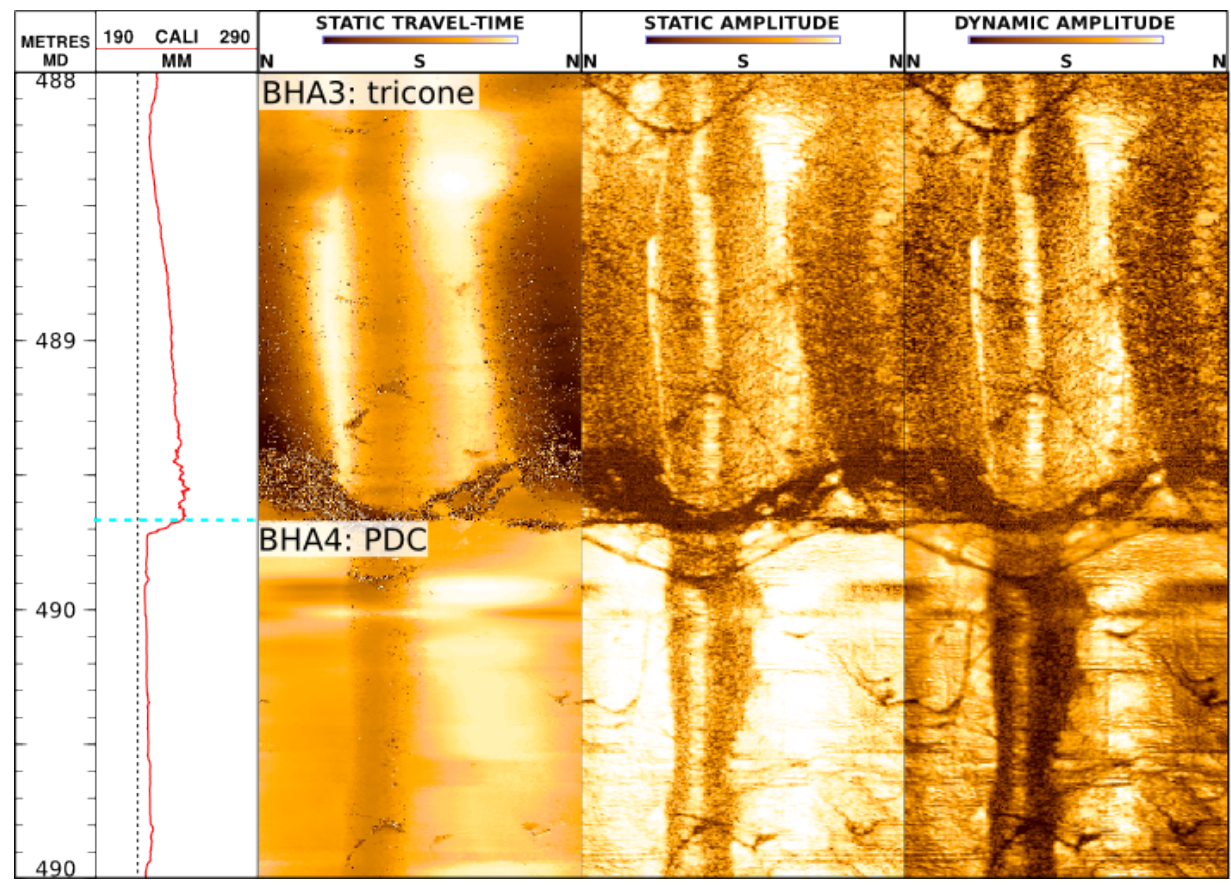

Figure 5.C.1: Impact of drill bit type on BHTV log appearance: detail of the appearance of the BHTV log with a tricone IADC 447 above $489.5 \mathrm{~m}$, and a PDC drill bit below. The boundary appears "flat" because it is perpendicular to the borehole trajectory. From left to right: borehole diameter calculated from travel-time and drill-bit size, statically normalised travel-time and amplitude images, dynamically normalised amplitude image.

grainy aspect. Figure 5.C.1 shows the limit between an interval drilled with a Tricone bit (BHA3) and a PDC bit (BHA4). The interval drilled with a PDC bit appears smoother and more circular than the Tricone-drilled interval, with a lower borehole diameter only $5 \mathrm{~mm}$ larger than the drill-bit size. The dual-rotary drill bit (264-276 m) made a smooth and circular borehole. BHA1 (PDC) yielded a smooth borehole, but rapid wear with depth (to $396.7 \mathrm{~m}$ ) decreased borehole smoothness and BHTV log quality. This interval has also been reamed by successive drill string trips in and out of the hole during fishing operations, which likely caused the excavation on the high side and a scraping on the low side of the borehole in the 316-476 m interval. This caused up to $50 \%$ of the image to be obscured. Rate of penetration of the PDC and tricone IADC 447 drill bits was slow, so hard-rock bits (tricone F7 and J8 bits) were used below $546 \mathrm{~m}$. While hard rock bits drilled significantly faster, the resulting borehole is less circular and smooth than with soft-rock bits, which decreased the BHTV log quality and added drilling-related artefacts (diagonal scratching, and blurry appearance likely caused by higher roughness of the borehole wall). The $558-598 \mathrm{~m}$ interval was logged at two different times with different mud temperatures (hence different window times). The bad BHTV log quality recorded in both cases confirms that the borehole shape and borehole roughness is an important cause for bad BHTV log quality at this depth. The combination of Tricone F7 and J8 with the use of the ABI43 and the smaller allowable 
window time, caused the overall decrease in image quality in the 546-893.2 $\mathrm{m}$ interval. The 797-828 m interval had to be reamed with the new bit of BHA7 because of wear on BHA6 drill bit and appears blurry (reaming actually occurred from $555 \mathrm{~m}$ but does not affect the R47 log, run before reaming).

Other standard artefacts are observed (Lofts and Bourke, 1999). A keyseat occurs intermittently between $470-600 \mathrm{~m}$, and systematically between $600-888 \mathrm{~m}$, on the low side of the borehole. Keyseats appear as a borehole-axial band of low acoustic amplitude and enlargement, located only on the low side of the borehole, contrary to breakouts which occur in pairs and opposed by $180^{\circ}$. An artefact caused by marks made by the BHA stabilizers is observed at various depths where drilling halted temporally. This artefact is characterised by $15 \mathrm{~mm}$ caliper increases over $0.4-1.9 \mathrm{~m}$ depth interval (depending on the type of stabilizer) and an out-of-focus image between two apparently flat borders (Figure 5.6). Logging-related artefacts include high tool rotation around its long axis which produces horizontal marks on the log. The $886-888 \mathrm{~m}$ interval is of bad quality, which is typical of the bottom of a borehole where viscous drilling mud sticks to the acoustic head of the tool and prevents any signal from being recorded. Changes of log run result in an apparently "flat" line (Table 5.A.1). 


\section{Synthesis}

This thesis presents a study of fracture systems in three contrasting geological settings. The following synthesis presents key findings arising from the analyses made in Chapters 25 and revisits the questions introduced in Chapter 1. Each study opened new avenues for research, and directions for future work are also suggested under the relevant sections.

\subsection{Main contributions}

This work studied in detail the fracture system geometries, factors controlling the formation of fractures, and implications of these fracture geometries and processes for permeability in an outcropping andesite lava flow of the Ruapehu volcano (Chapter 2), the andesite-hosted high-temperature Rotokawa Geothermal Field (Chapter 4), and the hangingwall of the Alpine Fault (Chapter 5). We have quantified fracture geometries using a combination of outcrop and Terrestrial Laser Scanner (TLS) datasets (Chapter 2); thin-section, cores and acoustic borehole televiewer (BHTV) logs (Chapter 4); and BHTV logs combined with relevant outcrop observations (Chapter 5). To this end, we have used statistical techniques to delineate fracture sets and evaluate the probability distributions of fracture geometries measured in BHTV logs (Chapter 3). We have also developed a workflow for analysing fracture systems extracted from a TLS point cloud (Chapter 2).

\subsubsection{Key Results of Each Chapter}

\section{Chapter 2; Fractures in an Outcropping Andesite Lava Flow}

- Fracture geometries in a blocky andesite lava flow of the Ruapehu volcano, New Zealand, were automatically extracted from a TLS dataset using a shapedetection algorithm.

- The fracture system comprises column-forming fractures either parallel (subhorizontal) or perpendicular (sub-vertical) to the brecciated lava flow margins. The fracture length distribution is exponential, indicating the existence of a characteristic scale.

- Clustered platy fractures sub-parallel to the lava flow direction arrest or deflect column-forming fractures. 
- Autobreccias are partially connected to column-forming fractures, and can either facilitate lateral fluid flow or form barriers to fluid flow, depending on subsequent hydrothermal alteration history.

- Areal and volumetric fracture intensity analyses suggest $\sim 0.5 \%$ connected fracture porosity, which fosters fluid flow via planarity, connectivity and ubiquity.

- Discrete fracture network models of the blocky interior of the lava flow, generated with fracture geometries obtained from the TLS dataset, yield a highly connected fracture system similar to that measured in detail in the manual outcrop scanline.

\section{Chapter 3: BHTV Fracture Data Analysis}

- Clustering algorithms separated fractures interpreted from BHTV logs acquired in the Rotokawa Geothermal Field by their orientation in a quantitative and transparent manner.

- Maximum likelihood estimates using truncated distributions, and statistical criteria, yield the functional form and corresponding parameters that best represent the distribution of fracture thickness and spacing affected by sampling biases, amongst a selection of relevant probability distribution forms. This methodology is applicable to other fracture geometrical parameters, such as length.

- We evaluated sampling bias of fracture orientation, thickness and spacing measured on BHTV logs. The variable log quality is accommodated by a compromise between sufficient detection and quantity of input data.

- We quantified the effects of location, size and range of the fully-sampled interval of geometrical parameter values on the possibility that several distributions approximate the data similarly well.

\section{Chapter 4: Controls on the Fracture System in the Rotokawa Geothermal} Field

- Fracture orientation, thickness and spacing were analysed in cores and BHTV logs in the andesite-hosted, high-temperature Rotokawa Geothermal Field.

- Fracture spacing of the predominant fracture orientation sets is log-normal, and interpreted to be controlled by mechanical stratification of the andesite reservoir. Two subordinate fracture sets have a power-law spacing interpreted to be controlled by tectonic faults.

- A single power-law distribution approximates fracture thickness in BHTV logs and cores but not in thin-sections. This change with scale is interpreted to reflect changes between isotropic (thermal) stress controls on fracture location and orientation at thin-section scale, and anisotropic (lithological/tectonic) controls at larger scales. 
- Fractures occupy $\sim 5 \%$ of the rock at three different scales, suggesting a selfsimilar behaviour of fracture volume in 3-D.

\section{Chapter 5: Structural Analysis of DFDP-2B BHTV logs}

- Metamorphic lithologies and structures were interpreted from BHTV logs in the Alpine Fault hangingwall, along a $630 \mathrm{~m}$-long section of the DFDP-2B borehole.

- Foliation observed in the BHTV logs has a constant orientation $\left(60^{\circ} / 145^{\circ}\right)$ similar to that measured in nearby outcrops and to the regional strike of the Alpine Fault, and suggests a similar Alpine Fault plane orientation at $\sim 1 \mathrm{~km}$ depth in the Whataroa Valley.

- Sub-vertical fractures striking NW-SE above $\sim 500 \mathrm{~m}$, and sub-horizontal below this depth, are interpreted to be exhumation-related joints and inherited hydrofractures, respectively.

- Brecciated fault zones similar to cataclasite zones observed in DFDP-1 drill cores and BHTV logs are found at multiple depths.

- The fault zones and three fracture sets (foliation-parallel, sub-vertical and subhorizontal) promote high connectivity in the hangingwall of the Alpine Fault, which may allow pervasive fluid flow.

\subsubsection{Key Contributions to the Research Questions}

\section{Fracture Characterisation in Non-sedimentary Layered Systems}

Chapter 2 quantified the fracture orientation, length, and 2-D density of columnforming fractures in an outcropping andesite lava flow. The observed scale-dependent fracture length is consistent with a layered system (Schöpfer et al., 2011). With appropriate assumptions, the 3 -D fracture density is estimated to represent $\sim 0.5 \%$ of the rock mass. Although small, this 3-D fracture density allows for a fully connected system, as demonstrated by discrete fracture network models. Simple calculations of permeability with a 3-D isotropic array of parallel fractures aligned with the flow, and with a parallel plate model (Phillips, 1991), yield a permeability of $\sim 6 \cdot 10^{-12} \mathrm{~m}^{2}$, which allows for convection (Rowland and Sibson, 2004). Such analysis could be extended to more complex geometrical configurations, such as constraining the role of non-flat and steeper paleo-topography, ice-lava contacts (Spörli and Rowland, 2006, Conway et al., 2015), or differences in chemical and mineralogical configuration (Hetényi et al., 2011).

In the Rotokawa geothermal reservoir (Chapter 4), the fracture set delineation study highlighted the presence of a fracture set striking parallel to $\mathrm{S}_{\mathrm{Hmax}}$ and dipping $60^{\circ}$, typical of an Andersonian normal faulting regime, in addition to other fracture sets 
previously interpreted by McNamara et al. (2015). Statistical analysis of fracture spacing suggests the presence of both layered- and fault-controlled fracture sets. While the layers themselves cannot be directly observed from available data, it is consistent with the geological setting of a sequence of andesite lavas and breccias as the one described in Chapter 2. This study also provided the first 1-D and 3-D fracture volume estimation at three scales of observation, which will be very useful to constrain fracture simulations. The 3-D $\sim 5 \%$ fracture volumes at thin-section-, core- and BHTV log-scales suggest a self-similar configuration in 3-D, although the fracture location, and likely length and connectivity are affected by scale-dependent layering. Further numerical models of fracture geometries and associated fluid flow would help reconciling these observations.

In the metamorphic hangingwall of the Alpine Fault (Chapter 5), fractures are dominantly parallel to foliation, with a constant orientation along the $630 \mathrm{~m}$-long section, which suggest that the Alpine Fault plane is oriented 60/145 (dip/dip direction) in the Whataroa Valley at $\sim 1 \mathrm{~km}$ depth. Subsidiary fracture sets (sub-vertical and sub-horizontal) and cataclasite zones provide a well-connected system.

For both studies based on borehole data (Chapters 4 and 5), fracture length distribution evaluations would be beneficial, either via numerical simulation constrained by the estimated fracture density and orientation (e.g., Davy et al., 2006), or by combining borehole- and near-borehole geophysics studies such as vertical seismic profiles (VSP; Place et al., 2011). In addition, spatial variations could be better constrained in each of the three studied settings, via e.g. analysis of new borehole or outcrop data, further analysis of fracture system's spatial variations along the entire length and section of a lava flow (instead of a flow-parallel section of a portion of a lava flow), or a statistical analysis of fractures in the Alpine Fault's hangingwall as described in Chapter 3.

\section{Advances of Fracture Characterisation Methods}

We have presented methods which advance the robust analysis of fracture geometries in non-sedimentary rocks, by modifying known methods to suit the particular cases of outcropping lava flows and borehole image logs. These advances can be applied to other settings.

In Chapter 2, we have presented a complete analysis workflow to extract fracture geometrical parameters from TLS datasets, allowing the generation of discrete fracture network models. Most currently-existing algorithms extracting fracture planes from TLS are not applicable to blocky lava flows which lack fracture sets of specific orientations. To our knowledge, we were the first to use the Facet shape detection algorithm, following the demonstration of the technique by Dewez et al. (2016). The additional constraining of Facet parameters by the Ransac algorithm on a subset of the point cloud, further calibrated by manual scanline measurements in parts of the outcrop, 
and panoramic photographs for the entire outcrop, proves the efficiency of the Facet algorithm and provides reliable estimates of fracture geometries. The fracture data analysis workflow presented in Figure 2.3 is a new advance which allows a comprehensive evaluation of fracture geometries. The fracture length and other key parameters evaluated are those required to generate discrete fracture network models, and more generally to constrain fracture models. Further improvements of this workflow would involve the automatic estimation of areal and volumetric fracture densities.

The statistical methods described in Chapter 3 yielded robust results for the study of fracture orientation, and scaling of thickness and spacing from BHTV logs in Chapter 4. The scaling analysis method is applicable to other attributes sampled either in boreholes or manual scanlines in outcrops, such as length. While the clustering and probability distribution fitting method is commonly used by statisticians, we have detailed here the various precautions and sampling biases to consider for BHTV logs, which is directly applicable to other types of borehole image logs and more generally to linear fracture sampling such as manual scanlines. This chapter is aimed to a geoscientific audience, which will help the uptake of this set of methods that are mathematically more robust than subjective manual delineation of planar feature sets on stereonets and least-square regressions of fracture attributes. As detailed in section 3.6.3 further improvements should involve multivariate analysis, with relevant metrics taking into account the relative importance of the attributes jointly studied, as well as spatial variations.

Following on Massiot et al. (2015), and due to the lack of BHTV log studies in foliated metamorphic rocks, we have designed an in-depth descriptive feature scheme for BHTV log analysis in the DFDP-2B borehole. This has allowed the interpretation of feature sets in terms of their genetic origin after comparison with outcrop analogue. In turn, other authors can use the detailed description scheme to provide alternative interpretations, or to further interpretation with e.g. multivariate analysis Townend et al., 2013), without the need to re-interpret the BHTV logs. The descriptive scheme can easily be tailored to other geological settings in non-foliated rock masses, or strictly applied in routine BHTV log analysis.

\section{Controls on Fracture System Architecture}

Figure 6.1 summarises the main controls on the fracture system architecture for the andesite lava flow studied on the Ruapehu volcano (Chapter 2), the Rotokawa andesitehosted reservoir (Chapter 4) and the epithermal deposit of Waihi, Coromandel Peninsula north of the Taupo Volcanic Zone (Brathwaite et al., 2001).

In Chapter 2, the layered nature of the lava flow is reflected in the measured scaledependent fracture length. Column-forming orientation without mean dip direction (but either parallel- or perpendicular to the lava flow direction) is consistent with the 


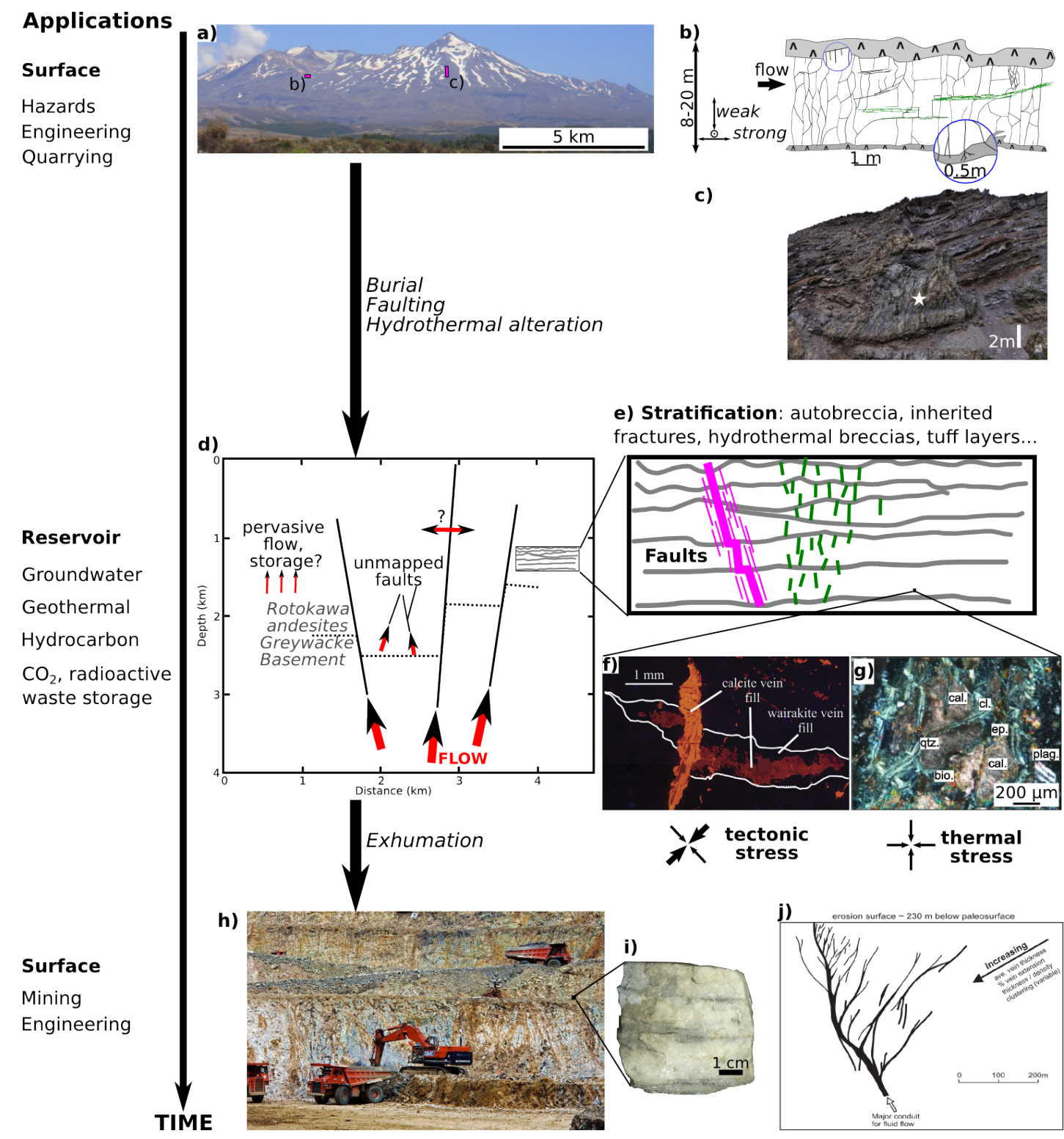

Figure 6.1: Possible evolution of volcanic rocks through depth and time. a) Ruapehu volcano. b) Summary diagram of fractures in the Happy Valley lava flow (Figure 2.12) with mechanical UCS anisotropy (Appendix A). c) Overthickened knuckle of lava (star) within sequence of alternating planar lava flows, autobreccias and debris flows in the Whangaehu Valley, Ruapehu (Conway et al., 2016). d) Summary diagram of the Rotokawa reservoir, with possible flow pathways through kilometre-scale faults and inferred smaller faults, and storage of fluid (or limited flow) in pervasive fracture network. e) Schematic stratification in an andesitic sequence influencing fracture location (green), and fractures associated with segmented faults (purple). f) Cathodoluminescence image of a calcite/wairakite-filled vein cross-cut by a younger calcite-filled vein of the Kawerau Geothermal Field, TVZ (McNamara et al., 2016b). g) Pervasive isotropically oriented fractures in thin section of the Rotokawa Geothermal Field (light blue; Siratovich et al., 2014). h) Waihi gold mine hosted in andesites (photograph by Lloyd Homer, GNS Science visual media library, https://vml.gns.cri.nz). i) Gold fleck in quartz vein sample (photograph by Lloyd Homer, GNS Science visual media library, https://vml.gns.cri.nz). j) Schematic representation of spatial variation of veining with depth and relative to major conduits of fluid flow, based on cross sections in the Martha Hill andesite-hosted deposit (Brathwaite et al., 2001). The location of enlargements in a-d-e-h is indicative of scale only. 
effects of the inwards propagation of the thermal gradient as modelled by Lore et al. (2000, 2001). In addition, platy fractures aligned with vesicular layers cause the arrest or deflection of column-forming fractures. In this simple configuration, the fracture system architecture is thus controlled by a combination of thermal and layering controls (Figure 6.1 a-c). Similar studies of other representative outcrops will undoubtedly open new perspectives on controls on these volcanic fracture systems at the scale of an entire volcano.

At Rotokawa, we have shown a combination of tectonic, lithological and thermal controls at different scales (Chapter 4): tectonic on fracture orientation, thickness, and spacing for some orientations at BHTV log scale; layering on fracture spacing at BHTV scale; and a combination of thermal and tectonic controls on fracture orientation at thin-section scale (Figure 6.1 $\mathrm{d}-\mathrm{g}$ ). While the location and thickness of mechanical layers cannot be directly observed from available data in the Rotokawa reservoir, they are suggested by the host lithology and indirect results of fracture spacing. Layering likely impacts the segmentation of the large faults (Figures $4.2,6.1 \mathrm{k}$ ), but is not resolved by stratigraphic offsets between nearby wells and microseismicity. The change of fracture orientation (mostly isotropic to anisotropic at thin-section and BHTV log scales, respectively) and different thickness scaling at thin-section scale compared to core- and BHTV log-scales, also suggest different processes affecting fractures at different scales. Studies aimed at recognising rock textures in borehole datasets, such as resistivity image logs (Halwa et al., 2013), may allow the delineation of hydrothermal breccias and individual lava flows, or other features causing the mechanical layering.

In the Waihi andesite-hosted epithermal deposit, the location of veins, and thus paleo-conduits for fluid flow, is dominantly associated with faulting (Figure 6.1 h-j). Column-forming fractures and veins such as those described in Chapter 2 are not always evident (Brathwaite et al., 2001). On the contrary, flow-banding and breccias have been observed in other epithermal deposits in Coromandel (Zuquim and Rowland, 2013). These observations are consistent with fluid flow in an andesite-hosted geothermal reservoir such as Rotokawa dominantly controlled by faults and their damage zones, but where lava flow sequences may also be preserved, which both affect the fracture system architecture.

In the hangingwall of the Alpine Fault, foliation is a major control on the location of fractures, as most inferred fractures are parallel to foliation planes. In addition, high paleo-pore pressure, which created the interpreted inherited hydrofractures at greater depth, and exhumation, which brought the inherited hydrofractures to the near-surface and generated the interpreted exhumation joints, affect the fracture system geometry. Finally, subsidiary faults, associated with cataclasite textures and high fracture density, reflect the accommodation of tectonic stresses away from the principal slip zone. The combination of these processes controlled the fracture system geometry and result in a potentially well-connected system. 


\section{Modelling of Fracture System Geometries}

In this thesis, we have used discrete fracture network models (DFNs) as examples of geometrical fracture models. DFNs are straightforward to generate, though large sets of random simulations are necessary to obtain reasonable estimates of model uncertainties (de Dreuzy et al., 2013). In combination with classic DFNs, fault down-scaling modelling (Julio et al., 2015), which automatically sub-divides single-plane faults, may be applicable to the hangingwall of the Alpine Fault where fault zones are observed (Chapter 5), or in the Rotokawa reservoir where such faults are inferred (Chapter 4 McNamara et al., 2015).

However, DFNs do not explicitly model the structures as observed in outcrops. For example, the blocky structure of the Ruapehu lava flow studied in Chapter 2 is not preserved in DFN models, as fracture centres are randomly distributed, which thus modifies the system connectivity. Hex-dominant meshes (Botella et al., 2015) may offer a viable approach to explicitly model the blocky structure of lava flows such as Happy Valley. This technique grids a volume with different primitive shapes, but dominantly hexahedra (elongated cubes). With the facets of each hexahedron representing column-forming fractures, hex-dominant meshes may accommodate the fracture geometries quantified at Happy Valley, including orientation (arising from the primitive blocky shape) and length (by adjusting grid size). However, even in the case of the well-defined Happy Valley lava flow, upscaling fracture systems from a single lava flow to an entire volcano remains non trivial due to the spatial variability of volcanic products (Chapter 2) and the high number of resulting cells in numerical models.

Geostatistical models are more generic as they represent the distribution of petrophysical parameters (such as fracture porosity and permeability) within a gridded volume, rather than fracture planes explicitly. DFN models may be used as a preliminary step in evaluating the connected fracture porosity and permeability, and their variability, within a representative elementary volume (Berkowitz, 2002). Geostatistical models can either be object-based, which preserves the general conceptual model (e.g, Viseur, 2004; Ruiu et al., 2015, for fluvial meandres), or pixel-based as extensively used in hydrocarbon reservoirs and in some geothermal reservoirs (Aprilina et al., 2015). A pixel-based approach loses the explicit link to the alternation of breccia and blocky zones of lava flow-hosted reservoirs, but can maintain the ratio of breccia to blocky volumes and characteristic lava flow scales (Pollyea et al., 2014). Furthermore, these ratios can be calibrated with TLS data analysis (Pollyea and Fairley, 2011). Investigation of the effect of lava flow thickness on fracture densities in outcrops would provide constraints on fracture densities within the representative elementary volumes. Additional modelling strategies may also be applicable, such as neural-network or pipe network models (de Dreuzy et al. 2013, and references therein).

The modelling strategy ultimately adopted depends on the desired outcomes in 
terms of scale, resemblance to the conceptual model, and its intended application (purely geometric, or involving solution of fluid flow or geomechanical equations). Meshing complex fracture geometries is a challenge, and modelling fluid flow in such complex meshes can quickly become too computationally expensive Kissling et al., 2015b). The amount, spatial distribution and accuracy of data used to constrain the models is also decisive in selecting the modelling approach. Pixel-based geostatistical models of the andesite-hosted Salak geothermal reservoir (Indonesia) were facilitated by numerous borehole measurements and core data (Aprilina et al., 2015). New data on fracture system geometries in volcanic and metamorphic rocks provided in this thesis offer new opportunities for modelling fluid flow in these settings. Most importantly, combining volcanological (e.g., Wilson and Charlier, 2016), hydrological (e.g., Custodio, 2007; Lachassagne et al., 2014) and geothermal (e.g., Hernandez et al., 2015) conceptual models can significantly improve the accuracy of the resulting fluid flow realisations.

\subsection{Implications for Future Research}

Here, we expand on and discuss some of the implications of the key findings of this research. Over the course of this study, many other questions and possible lines of further enquiry arose that could be addressed by future work, and relate to three overarching questions:

1. What is the effect of past geological events and associated physical properties (petrophysical, mechanical and thermal) on fracture systems, on different spatial and temporal scales?

2. What are the links between fracture system geometry and permeability in a particular stress field?

3. How can we best model and constrain fracture systems geometries and the resulting permeability at reservoir scales, using limited observations?

\subsubsection{Role of Past Geological History on Current Fracture System Geometry}

Mechanical anisotropy caused by layering at various scales, such as observed at Ruapehu (Chapter 2, Appendix A), inferred in the Rotokawa reservoir (Chapter 4), or inherent to foliated rocks in the hangingwall of the Alpine Fault, increases the complexity of the fracture system by affecting fracture location and propagation (Figure 6.1., e; Schöpfer et al., 2011; Misra et al., 2015). Fault segmentation, which can be promoted by mechanical anisotropy or the coalescence of pre-existing fractures (Crider, 2015), 
localises fluid flow at the reservoir scale. These concentrations of fluids define targets for geothermal drilling, or for blind geothermal field exploration which lack surface evidence of an hydrothermal system (Siler et al., 2016). The relationships between anisotropy of the host lithology and fracture propagation should be further explored in settings of varied mechanical anisotropy, layer thickness, and thermodynamic conditions. Promising recent advances include the quantification of a Hoek-Brown failure criterion for the Rotokawa andesites, better suited than a Mohr-Coulomb criterion to mechanically describe such pervasively fractured rocks (Siratovich et al., 2016).

The contrast between the layering formed by the successive addition of lava flow and other volcaniclastic formations on an active volcano (Chapter 2), and the lack or light remnant of layering in volcanic-hosted epithermal deposits, offers end-member observations to study processes occurring in sub-surface geothermal systems where data is sparse. Refining the links between fracture systems at each stage is important for understanding how geothermal systems evolve temporally, with practical consequences for managing contemporary geothermal resources. We propose a few hypotheses which may contribute to this radical change of architecture, and require further testing by e.g. numerical simulations:

Pervasive alteration processes: Pervasive hydrothermal alteration, such as observed on some cores at Rotokawa (Figure 4.3), may homogenise the mechanical properties of the rock mass. Indeed, rock tests on unaltered samples from the Happy Valley lava flow (Appendix A indicate a strength anisotropy, with samples parallel to the lava flow direction having a higher unconfined compressive strength than those perpendicular to the lava flow direction. On the contrary, altered samples from Rotokawa did not demonstrate clear strength anisotropy Siratovich et al., 2014).

Stress effects: The burial and associated increase in vertical stress, combined with the ongoing rifting and associated faulting, likely preferentially open or close the original column-forming fractures, depending on their orientation within the stress field. While fractures formed during the cooling of lava flow may still be present after burial, they may not be sufficiently open to be detected or to conduct fluid flow. In addition, increased pore pressure can maintain open those fractures perpendicular to $\sigma_{3}$ (minimum principal stress) or induce shearing (Sibson, 1992, Hickman et al., 1995; Barton et al., 1995). Finally, thermal stress affects the rock mass isotropically, which may reinforce the effects of pervasive alteration.

Fracture growth by coalescence of joints: The difference in fracture thickness at Ruapehu ( $\sim 2 \mathrm{~mm}$ ) Rotokawa (up to several centimetre as detected on BHTV $\operatorname{logs}$ ) and veins in epithermal deposits (up to several metres), suggests a continuous thickening process. Fracture growth by coalescence of column-forming frac- 
tures Crider, 2015) could generate vein architecture such as the one described by Brathwaite et al. (2001) displayed in Figure 6.1.].

The temporal and spatial scales over which mechanical anisotropy is diminished from the outcropping lava flows to the volcanic-hosted epithermal deposits (Brathwaite et al., 2001; Custodio, 2007) is important for characterising fracture system geometry and associated fluid flow in geothermal systems. The preferential closing or opening of these column-forming fractures within a state of stress and fluid flow circulations could be tested via numerical simulations (Kissling et al. 2015b). Systematic comparison of cores and borehole image logs (acoustic or resistivity) are rare in volcanic and metamorphic fractured rock masses, although they would provide necessary constraints to interpret fracture type (opening or shearing mode) and mineralogy, as well as possible layering in image logs. The combination of core, borehole- and near-borehole geophysics, in the light of outcrop studies and numerical simulations, would thus help understand how much of the initial volcanic architecture is preserved during burial (or exhumation) and hydrothermal evolution.

Metamorphic rocks observed in the DFDP-2B borehole are also at an intermediate stage of exhumation, and while detailed fracture studies have been conducted on outcrops (e.g., Gillam et al., 2013), observations at depth are limited to the DFDP boreholes (Townend et al., 2013; Williams et al., 2016) and indirect seismological observations (e.g., Boese et al., 2012). The deformation of schists into protomylonite and higher grades of deformation, together with similar stress and pore pressure effects as those described for the Rotokawa reservoir, also need to be further investigated.

\subsubsection{From Observed Geometry to Connectivity, Stress and Fluid Flow}

The density and, in particular, the connectivity of fractures affect the degree of permeability enhancement associated with fractures. Figure 6.2 presents simple examples of fluid flow variations caused by fractures, with matrix and fracture petrophysical properties analogous to the Rotokawa reservoir Kissling et al., 2015b, and references therein). These models are applicable to any length scale, as they only rely on the proportion of fracture volume, fracture distribution and ratio of fracture to matrix permeability. A single fracture aligned parallel to the direction of flow, and spanning the model domain from lower to upper boundary, increases permeability by $\sim 300 \%$. By contrast, increasing the density of unconnected fractures oriented parallel to the main flow path increases permeability only marginally by up to a few tens of percent (Figure 6.2 a, c). Randomly oriented fracture networks create complex permeability variations at short length scales ( $<10 \%$ of fracture length), and the bulk permeability increase of the fracture network can be moderate even when the connected fractures are oriented 
a) Flow-parallel, unconnected network

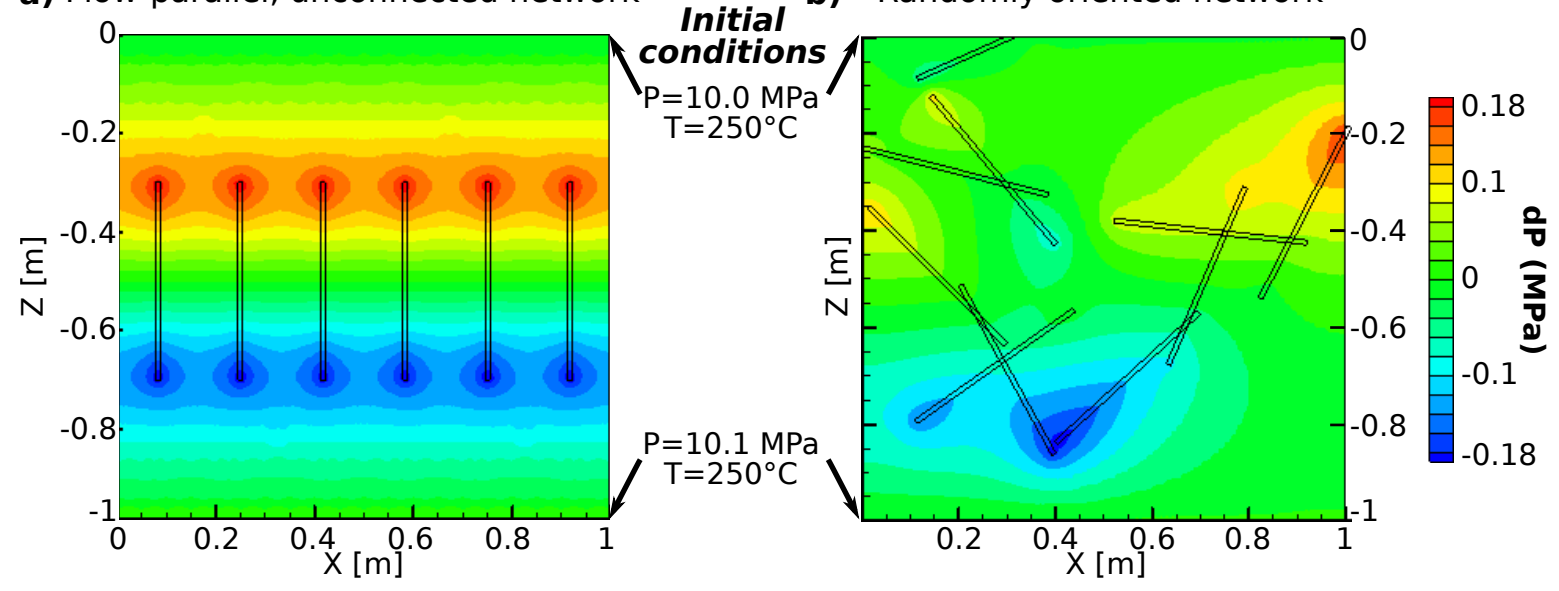

c)

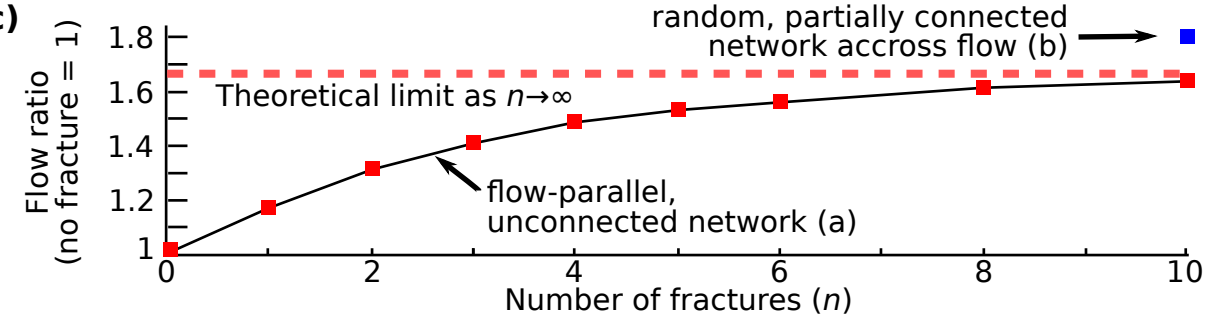

Figure 6.2: Numerical flow models at Rotokawa reservoir conditions with varying fracture density and orientation (modified from Kissling et al., 2015b). a) Fractures are unconnected and parallel to fluid flow. b) Fracture are randomly oriented, resulting here in a partially-connected network predominantly oriented perpendicular to fluid flow. c) Flow ratio (ratio of the mass flow across a fractured model domain to that in the same domain with no fractures) for both cases. In the case of the unconnected parallel fracture network, flow ratio has a limit of $\sim 1.67$ (formula in Kissling et al., 2015b). A single fracture oriented parallel to flow and connecting the whole domain has a flow ratio of $\sim 300$. In these models, matrix permeability $\left(\sim 10^{-17} \mathrm{~m}^{2}\right)$ is much lower than fracture permeability $\left(\sim 10^{-12} \mathrm{~m}^{2}\right)$. 
perpendicular to the main flow directions (Figure 6.2 b-c). Subordinate fracture sets identified in the Rotokawa andesite (Chapter 4), and in the Alpine Fault hangingwall (Chapter 5), may significantly increase the connectivity of the fracture system, and, although representing a small portion of the overall system, may increase and spatially concentrate fluid flow. In contrast, the pervasive fracture system observed in cores and BHTV logs in the Rotokawa reservoir may offer storage in-between zones of measured permeability increases (Figure 6.1d).

As illustrated in Chapter 4, fractures which have different orders of magnitude of thickness can occupy the same volume of rock. However, thin and short fractures such as those observed in thin-section (Figure 6.1 f-g) can only have small contributions to permeability by themselves (Siratovich et al., 2014$)$, and are less likely to be interconnected than longer fractures (Bour and Davy, 1998). In addition, linkage of these short fractures likely yields tortuous fracture paths which may decrease their permeability potential (Heap et al., 2014; Heap and Kennedy, 2016). However, coalescence of microcracks under stress can increase permeability prior to forming macrofractures, which ultimately produces permeable faults (Mitchell and Faulkner, 2008). Conversely, kilometre-scale faults are known to act as both conduits or barriers to fluid flow, and a high fracture volume does not necessarily equate to a high permeability (Figure 6.1d, Caine et al., 1996; Rowland and Simmons, 2012). Quantifying the scaling relationships of fracture geometries provides a means of predicting the locations of fractures away from observations and evaluating possible flow pathways. In addition, techniques such as vertical seismic profiling can extend observations to a few hundreds of metres from a borehole, and help evaluate fracture connectivity (Place et al. 2011$)$.

In this thesis, we have described "static" contemporary fracture geometries, although these geometries likely vary in both space and time. Three aspects should be particularly considered in the settings studied in this thesis. Firstly, the transition from a geometrical model to a fluid flow model for a particular state of stress is a wide domain of research (e.g., Townend and Zoback, 2000, Baghbanan and Jing, 2008, Pollyea et al., 2014). Inherited fractures have arbitrary orientations with regard to the contemporary stress tensor, which affect their present-day propagation and mechanical behaviour, and hence permeability. For example, at the scale of an individual fracture, Figure 6.3 shows a synthetic example of two fractures under extension in conditions similar to those of the Rotokawa reservoir (Kissling et al., 2015b). The fracture initially perpendicular to the extension direction propagates slowly, with little impact on stress and permeability. In contrast, the fracture originally oblique to the extension direction develops a "wing crack" shape, with a large shearing zone, and 3.6 times more variation of pressure than the extension-perpendicular fracture. Secondly, water-rock interactions have fast kinetics in geothermal reservoirs (Sonney and Mountain, 2013), with similar processes likely to occur in the Alpine Fault hangingwall near the fault plane (the Rotokawa reservoir and DFDP-2B have thermal gradients exceeding $100^{\circ} \mathrm{C} / \mathrm{km}$; 


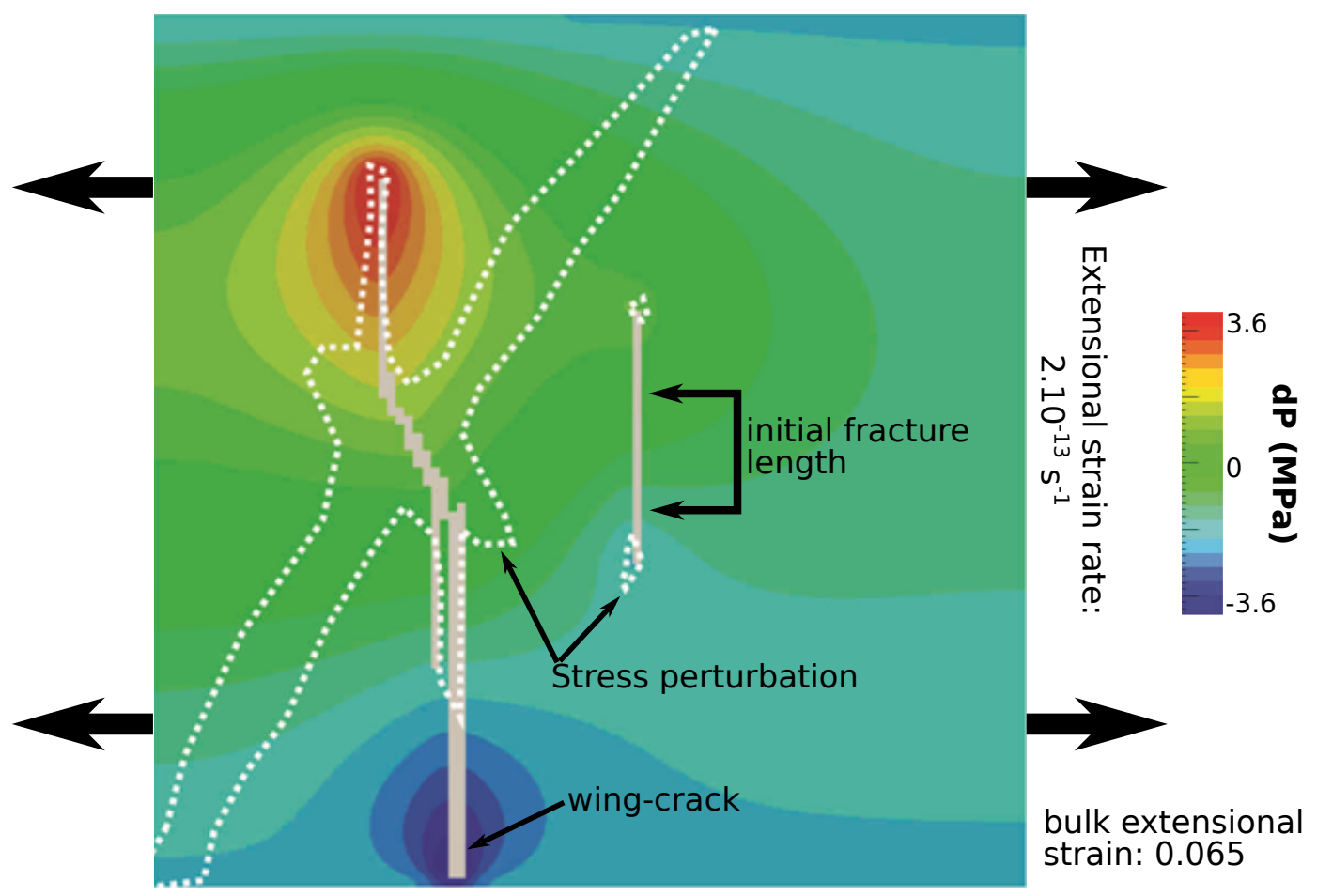

Figure 6.3: Effects of fracture orientation on stress and fluid flow under extension, with fractures initially oriented parallel (on the right) or at a $45^{\circ}$ angle (on the left) to the maximum stress orientation (modified from Kissling et al., 2015b). The fracture initially misoriented to the extension direction undergoes fracture propagation and interaction with a shear zone that nucleates at the left-lower corner of the model, and causes major fluid pressure perturbation. Same initial pressure and temperature conditions as in Figure 6.2.

Sewell et al., 2012; Sutherland et al. 2015). Thirdly, water-rock interactions are further enhanced in chemically reactive volcanic rocks (Custodio, 2007; Crider, 2015). Ongoing research aimed at coupling fracture geometries, mechanical properties, water/rock interactions and fluid flow offers prospects to understand better fluid flow pathways in these reservoirs. Development of this research field is hampered by computational issues arising from the 3-D (Sutherland et al., submitted), multi-scale (Kissling et al., 2015b) properties of the system, and limited available constraints from sparse data acquired in 1- or 2-D.

\subsubsection{Finding Constraints on Permeability}

In general, only a subset of fractures contributes to permeability in a reservoir $(M c N a-$ mara et al., 2015), either due to a lack of connectivity Bour and Davy, 1998; Kissling et al. 2015b) or because their hydraulic aperture is minimal. As an example of the latter, $70 \%$ of fractures in cores from the Rotokawa andesites appear fully infilled (Chapter 4). In boreholes, wireline measurements including pressure, temperature and flow velocity (PTS) logs indicate permeable zones Grant and Bixley, 2011; McNamara 
et al. 2015), although only a subset of fractures located within these 1-100 m-thick zones may significantly contribute to fluid flow (Mclean and McNamara, 2011). Joint interpretation of BHTV and PTS logs with other types of wireline logs (such as presented by Wallis et al., 2012b) can help characterising properties of conductive fractures, in conjunction with multivariate analysis as described in Chapter 3 .

Ongoing work by the DFDP-2 science team aims to locate permeable fractures and their characteristics in the DFDP-2B borehole. This work also has the potential to constrain the mineralisation type and intensity of fractures interpreted from BHTV logs using resistivity and sonic logs. In addition, exploring the possible relationships between identified permeable fractures and high shear:normal stress ratio, such as described by Barton et al. (1995) and Townend and Zoback (2000), may constrain stress conditions within the first 1 to $2 \mathrm{~km}$ below the ground surface. However, connectivity promoted by the subordinate fracture sets, or possible local stress changes near metre-thick fault zones (Chapter 5) may also play a significant role in the permeability distribution.

Constraints on kilometre-scale permeability and heat flow models in the Rotokawa reservoir, typical of geothermal reservoirs, consist of indirect measurements at different spatial scales, which are a challenge to use jointly. These measurements include pressure drawdown histories (Hernandez et al., 2015); tracer tests (Addison et al., 2015); microseismicity (Sherburn et al., 2015); natural state temperature modelling (Sewell et al., 2012); magneto-telluric models (Bertrand et al., 2015); and 3-D seismic velocity and attenuation models (Bannister et al. 2015). Near the Alpine Fault, regional-scale models can be constrained by measured rainfall, hot spring output, and inferred fluid flow from depth (Menzies et al., 2014; Sutherland et al., submitted). Laboratory permeability measurement of rocks from Ruapehu, Rotokawa andesites and Alpine Fault rocks are very valuable (Heap and Kennedy, 2016, Siratovich et al., 2014, Carpenter et al. 2014, respectively), although scaling issues need to be considered. Linking seismic b-values (the proportion of small to large earthquakes) and precise earthquake locations (Hopp et al. 2016) to scaling and location of fractures undergoing slip (and hence potentially permeable) is a promising research field (Barton et al., 2013; Ishibashi et al. 2016). This link may provide in-situ information away from boreholes to complement information provided in this thesis, and at depths unreachable by drilling. 


\subsection{Concluding Statement}

This study has characterised fracture systems and their potential impacts on permeability in an outcropping andesite lava flow, an andesite-hosted high-temperature geothermal reservoir, and the hangingwall of the Alpine Fault. We have used a variety of datasets at different scales, and advanced analytical techniques to characterise these fracture systems, while considering their specific sampling biases. Our work provides a framework for further studies of permeability in similar environments, both in New Zealand and overseas. This study highlights the role of host lithology on fracture system architecture, and forms the basis for continued research into what controls fracture nucleation and propagation in volcanic, metamorphic, geothermal and active fault environments. 
Appendices 


\section{A Physical Properties of Samples from the Happy Valley Andesitic Lava Flow and Meads Wall Dyke, Ruapehu Volcano, New Zealand}

We measured physical properties of two rock samples: one from the Happy Valley outcrop studied in Chapter 2, and another from the nearby Meads Wall dyke, near Iwikau Village on the northwest flank of the Ruapehu volcano. The Happy Valley lava flow was emplaced over a gentle paleo-topography, and sub-horizontal vesicular layers are observed with the naked eye. The Meads Wall dyke was emplaced sub-vertically and does not present any macroscale layering. The collected samples were bounded by natural macrofractures and did not show any macrofractures within them. In order to evaluate possible anisotropy of the measured rock properties core plugs of $\sim 25 \times 50 \mathrm{~mm}$ were drilled within each sample in two directions: parallel and transverse to the original lava flow direction. Therefore, "parallel" samples are sub-horizontal at Happy Valley and sub-vertical at Meads Wall, and vice versa for "transverse".

Density, connected porosity, and compressional and shear wave velocities, were measured on 13 core plugs. Unconfined compressive strength was measured on 8 of these samples. Sample preparation and property measurements were made in similar conditions to Siratovich et al. (2014) at the University of Canterbury (New Zealand) laboratory, which are briefly reiterated here.

\section{A.1 Methods}

\section{A.1.1 Density and Connected Porosity Measurements}

For density and connected porosity measurements, bulk volumes were obtained by caliper measurement (to within $0.01 \mathrm{~mm}$ ) and pore volume by water saturation. Once the samples were cut and ground flat and parallel, they were washed with water to remove any debris. Samples were then immersed in distilled water under a vacuum of about $100 \mathrm{kPa}$ for $24 \mathrm{~h}$, then taken out of the water and weighed after removing any surface water. The samples were then placed into a laboratory oven at $105^{\circ} \mathrm{C}$ and dried until a constant mass was observed. The connected porosity and dry bulk 
density of the samples were calculated following the methods recommended by Ulusay and Hudson (2007).

\section{A.1.2 Characterisation of Elastic Wave Velocities and Dynamic Elastic Moduli}

The compressional wave $(\mathrm{Vp})$ and shear wave $(\mathrm{Vs})$ velocities and dynamic elastic moduli were measured using a GCTS (Geotechnical Consulting and Testing Systems, Tempe, AZ, USA) Computer Aided Ultrasonic Velocity Testing System (CATS ULT-100) apparatus with axial $\mathrm{P}$ - and S-wave piezoelectric transducers.

The resonance frequency of the transducers was $900 \mathrm{kHz}$, pulse acquisition rate was $20 \mathrm{MHz}$, and multiple waveforms were captured for each sample. The velocities were collected under a constant uniaxial stress of $5 \mathrm{MPa}$ to ensure a consistent waveform across the specimens. Using these data, we determined the dynamic Poisson's ratio and Young's modulus using Equations A.1 and A.2, respectively Guéguen and Palciauskas, 1994):

$$
\begin{gathered}
V_{d}=\left(V_{p}^{2}-2 V_{s}^{2}\right) /\left(2\left(V_{p}^{2}-V_{s}^{2}\right)\right) \\
E_{d}=\left(\rho V_{s}^{2}\left(3 V_{p}^{2}-4 V_{s}^{2}\right)\right) /\left(V_{p}^{2}-V_{s}^{2}\right)
\end{gathered}
$$

where $V_{p}$ is the compressional wave velocity in metres per second, $V_{s}$ is the shear wave velocity in metres per second, $E_{d}$ is the dynamic Poisson's ratio, $E_{d}$ is the dynamic Young's modulus in pascal and $\rho$ is the density in kilograms per cubic metre.

\section{A.1.3 Uniaxial Compressive Strength Testing and Static Elas- tic Moduli Calculations}

Uniaxial compressive strength (UCS) was determined using a Technotest 3,000 kN servo-controlled loading frame (Siratovich et al., 2014, and references therein). Two $20 \mathrm{~mm}$-long strain gauges were glued onto each sample. One vertical gauge measures axial strain and one laterally-oriented gauge measures radial strain; care was taken to ensure that the strain gauges were perpendicular to their respective axes of deformation. The specimens were deformed at a constant strain rate of $0.22 \mathrm{~N} . \mathrm{mm}^{-2} \cdot \mathrm{s}^{-1}$ and 0.4 to $0.55 \mathrm{~N} . \mathrm{mm}^{-2} \cdot \mathrm{s}^{-1}$ for Meads Wall and Happy Valley samples, respectively (controlled by linear variable differential transformer, LVDT) at ambient laboratory temperature and humidity conditions. In the absence of pre-existing data for Ruapehu rocks, the applied strain rate was adapted accordingly after the first test of each sample to ensure that failure happened within 5-10 min and thus comply with ISRM requirements.

During experimentation, acoustic emission (AE) output was monitored using Physical Acoustics Corporation MISTRAS AE node acquisition system (Princeton Jct, NJ, 
USA). One physical acoustic WS $\alpha$ AE transducer (100 to $900 \mathrm{kHz}$ operating frequency) was attached to the samples in the middle, and recorded hit counts, waveforms, energy, and amplitude of the received signals during sample deformation. AE monitoring was used during deformation as a proxy for microcracking as AEs are generated by the release of energy from a material during the propagation and nucleation of microcracks (Eberhardt et al., 1998; Diederichs et al., 2004). We utilised arbitrary AE energy units (the area under the received waveform signal) for comparison of AE activity across the datasets. Once stress-strain curves were obtained, we calculated the static elastic moduli for each specimen utilizing Equations A.3 and A.4 with the tangent deformation modulus at $50 \%$ of the maximum peak stress (Ulusay and Hudson, 2007):

$$
\begin{gathered}
E_{s}=\Delta \sigma_{a} / \Delta \epsilon_{a} \\
\nu_{s}=-\left(\Delta \epsilon_{r} / \Delta \epsilon_{a}\right)
\end{gathered}
$$

where $E_{s}$ is the static Young's modulus $(\mathrm{Pa}), \nu_{s}$ is the static Poisson's ratio, $\sigma_{a}$ is the differential axial stress $(\mathrm{Pa}), \epsilon_{a}$ is the axial strain, and $\epsilon_{r}$ is the radial strain.

\section{A.2 Results}

Results of the petrophysical measurements are presented in Table A.1 with key crossplots in Figure A.1. Note the higher UCS values for lava flow-parallel compared to lava flow-transverse samples at Happy Valley (Figure A.1k), which indicates a strength anisotropy. The stress-strain curves and AE are displayed in Figure A.2. All samples displayed brittle failure. The acoustic emission curves of Happy Valley samples are characteristic of brittle behaviour with rapid closure of cracks followed by a plateau and ending with high energy emission at failure (Figure A.2. 
Table A.1: Physical property measurements of samples from Happy Valley lava flow (Hv) and Meads Wall dyke (Mw).

\begin{tabular}{ccccccccccc} 
Site & Dir & $\begin{array}{c}\boldsymbol{\rho} \\
\mathrm{g} / \mathrm{cm}^{3}\end{array}$ & $\begin{array}{c}\mathbf{n} \\
\mathrm{vol} \%\end{array}$ & $\begin{array}{c}\mathbf{V}_{\mathbf{p}} \\
\mathrm{m} / \mathrm{s}\end{array}$ & $\begin{array}{c}\mathbf{V}_{\mathbf{s}} \\
\mathrm{m} / \mathrm{s}\end{array}$ & $\begin{array}{c}\mathrm{UCS} \\
\mathrm{MPa}\end{array}$ & $\begin{array}{c}\mathbf{E}_{\mathbf{s}} \\
\mathrm{GPa}\end{array}$ & $\begin{array}{c}\mathbf{E}_{\mathbf{d}} \\
\mathrm{GPa}\end{array}$ & $\boldsymbol{\nu}_{\boldsymbol{s}}$ & $\boldsymbol{\nu}_{\boldsymbol{d}}$ \\
\hline $\mathrm{Hv}$ & $\mathrm{T}$ & 2.62 & 3.72 & 2,957 & 1,848 & 176.09 & 35.2 & 21.1 & 0.23 & 0.18 \\
$\mathrm{Hv}$ & $\mathrm{T}$ & 2.58 & 4.58 & 2,677 & 1,773 & 126.29 & 29.6 & 18.0 & 0.26 & 0.11 \\
$\mathrm{Hv}$ & $\mathrm{T}$ & 2.59 & 3.91 & 3,198 & 2,001 & & & 24.5 & & 0.18 \\
$\mathrm{Hv}$ & $\mathrm{P}$ & 2.68 & 2.15 & 3,064 & 1,971 & 242.17 & 46.5 & 23.9 & 0.38 & 0.15 \\
$\mathrm{Hv}$ & $\mathrm{P}$ & 2.71 & 2.68 & 3,157 & 2,026 & 236.34 & 43.8 & 25.6 & 0.35 & 0.15 \\
$\mathrm{Mw}$ & $\mathrm{P}$ & 2.21 & 8.50 & 3,544 & 2,170 & 104.23 & 31.0 & 25.0 & 0.27 & 0.20 \\
$\mathrm{Mw}$ & $\mathrm{P}$ & 2.22 & 9.50 & 3,658 & 2,151 & 84.60 & 39.8 & 25.4 & 0.75 & 0.24 \\
$\mathrm{Mw}$ & $\mathrm{P}$ & 2.26 & 8.08 & 3,774 & 2,222 & & & 27.6 & & 0.23 \\
$\mathrm{Mw}$ & $\mathrm{P}$ & 2.28 & 8.23 & 3,557 & 2,183 & & & 26.0 & & 0.20 \\
$\mathrm{Mw}$ & $\mathrm{T}$ & 2.32 & 8.58 & 3,269 & 2,074 & 96.28 & 22.8 & 23.2 & 0.3 & 0.16 \\
$\mathrm{Mw}$ & $\mathrm{T}$ & 2.31 & 7.94 & 3,426 & 2,112 & & & 24.6 & & 0.19 \\
$\mathrm{Mw}$ & $\mathrm{T}$ & 2.31 & 8.05 & 3,431 & 2,194 & 92.49 & 28.1 & 25.7 & 0.41 & 0.15 \\
$\mathrm{Mw}$ & $\mathrm{T}$ & 2.32 & 8.28 & 3,426 & 2,173 & & & 25.5 & & 0.16 \\
\hline
\end{tabular}

Dir: direction of sample, either transverse $(\mathrm{T})$ to lava flow/dyke direction or parallel $(\mathrm{P})$ to lava flow/dyke direction. $\rho$ : bulk dry density; $n$ : connected porosity; $V p$ : compressional wave velocity; $V_{S}$ : shear wave velocity; $E_{s}$ : static Young's modulus; $E_{d}$ : dynamic Young's modulus; $\nu_{s}$ : static Poisson's ratio; $\nu_{s}$ : dynamic Poisson's ratio.
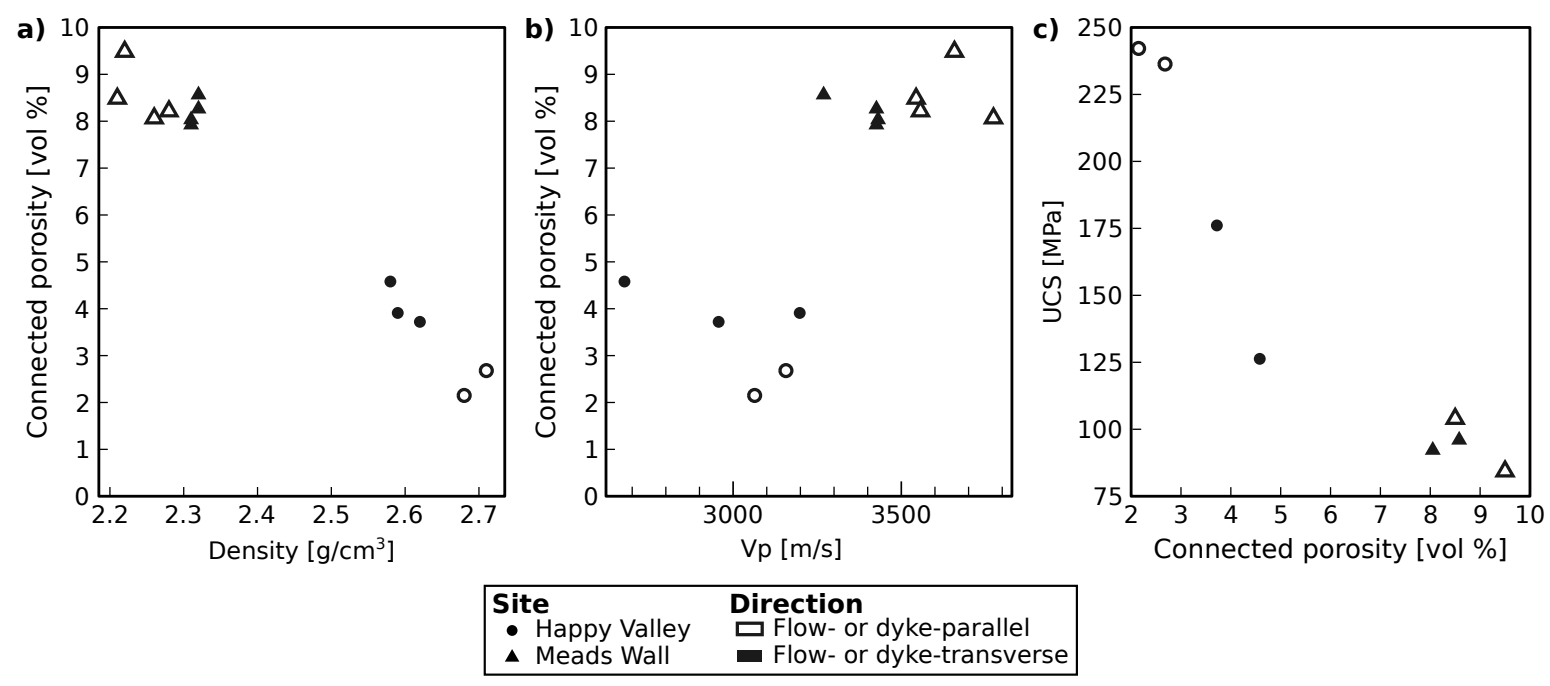

Figure A.1: Key crossplots of physical properties of Happy Valley (circles) and Meads Wall (triangles) samples. Open symbols are for samples parallel to the dyke- or lava flow-direction; filled symbols are for samples transverse to the dyke- or lava flowdirection. a) Density plotted versus connected porosity. b) Connected porosity plotted versus axial compressional wave velocity $(\mathrm{Vp})$. c) UCS plotted versus connected porosity. 


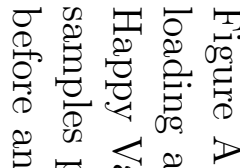
है

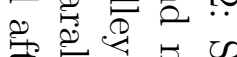
क्ष दड की है

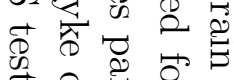
๑

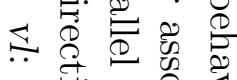
ङ

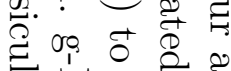

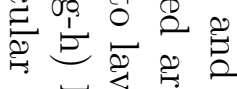

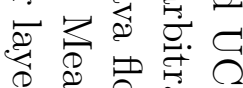
की है की की

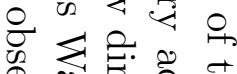

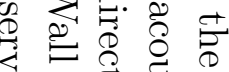
\& के ज्ञ त उ. \& $\frac{0}{8}$

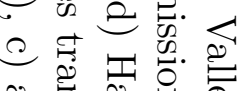

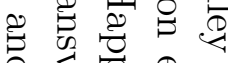

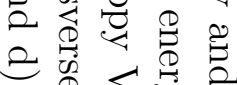
$\Omega$ के $\therefore$ 选龵 उ क्षे 节点

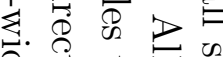
吉. ज़ है ?ํ. 요용 0

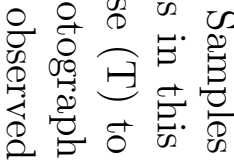
$\circ$ क क्ष 20 के

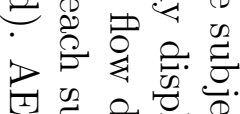
फ. 훙

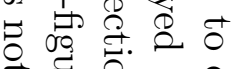
+ 过 0 芦

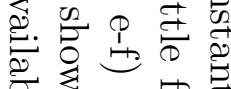
过家.

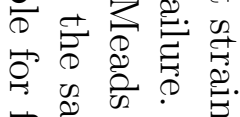
$\oplus \sum_{0}^{\infty} \sum_{0}^{\infty}$

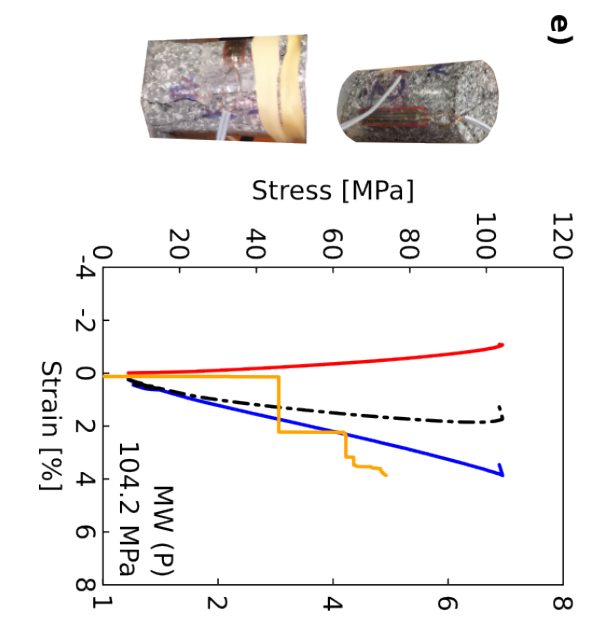
ข
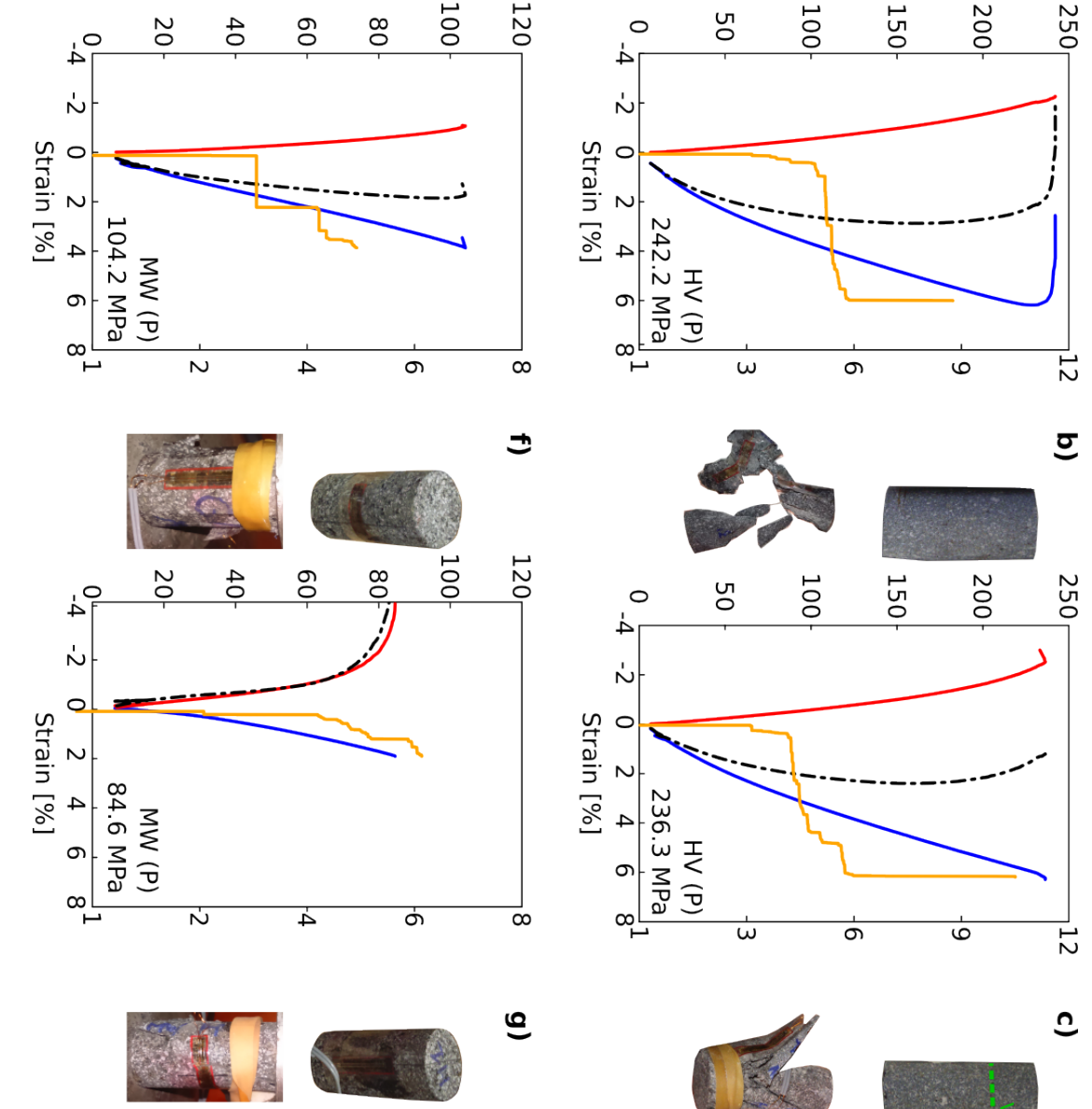

$\varrho$
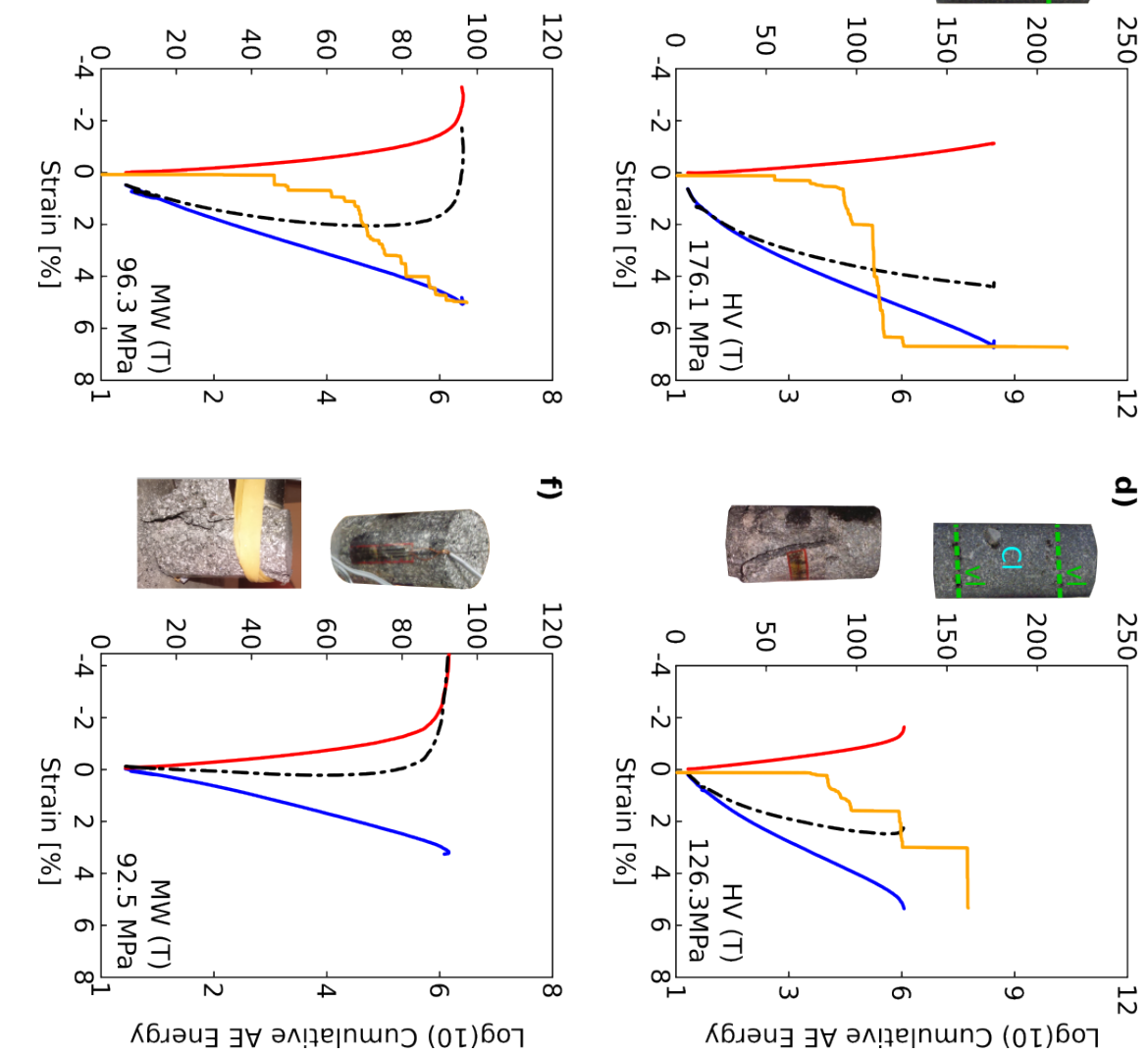

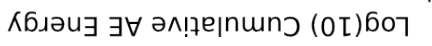




\section{Bibliography}

Abellan, A., M.-H. Derron, and M. Jaboyedoff (2016), "Use of 3d point clouds in geohazards" special issue: Current challenges and future trends, Remote Sensing, 8(2), 130, doi:10.3390/rs8020130.

Ackermann, R. V., R. W. Schlische, and M. O. Withjack (2001), The geometric and statistical evolution of normal fault systems: an experimental study of the effects of mechanical layer thickness on scaling laws, Journal of Structural Geology, 23(11), 1803-1819, doi:10.1016/s0191-8141(01)00028-1.

Addison, S., J. Winick, B. Mountain, and F. Siega (2015), Rotokawa reservoir tracer test history, New Zealand Geothermal Workshop 2015 Proceedings.

Agliardi, F., G. Crosta, F. Meloni, C. Valle, and C. Rivolta (2013), Structurallycontrolled instability, damage and slope failure in a porphyry rock mass, Tectonophysics, 605, 34-47, doi:10.1016/j.tecto.2013.05.033.

Agosta, F., M. Alessandroni, M. Antonellini, E. Tondi, and M. Giorgioni (2010), From fractures to flow: A field-based quantitative analysis of an outcropping carbonate reservoir, Tectonophysics, 490(3-4), 197-213, doi:10.1016/j.tecto.2010.05.005.

Akaike, H. (1974), A new look at the statistical model identification, IEEE Transactions on Automatic Control, 19(6), 716-723, doi:10.1109/tac.1974.1100705.

Alemdag, S. (2015), Assessment of bearing capacity and permeability of foundation rocks at the Gumustas Waste Dam Site (NE Turkey) using empirical and numerical analysis, Arabian Journal of Geosciences, 8(2), 1099-1110, doi:10.1007/s12517-0131236-3.

Aler, J., J. D. Mouza, and M. Arnould (1996), Measurement of the fragmentation efficiency of rock mass blasting and its mining applications, International Journal of Rock Mechanics and Mining Sciences $\&$ Geomechanics Abstracts, 33(2), 125-139, doi:10.1016/0148-9062(95)00054-2.

Andersen, L. (2011), A comparison of buried andesites at Ngatamariki and Rotokawa geothermal fields, Taupo, Msc, The University of Waikato, New Zealand. 
André-Mayer, A.-S., and J. Sausse (2007), Thickness and spatial distribution of veins in a porphyry copper deposit, Rosia Poieni, Romania, Journal of Structural Geology, 29(10), 1695-1708, doi:10.1016/j.jsg.2007.06.010.

Aprilina, N. V., D. Y. Satya, S. Rejeki, G. Golla, and M. Waite (2015), Geologic Modeling Workflow for Volcanic Hosted Geothermal Reservoirs: Case Study from Salak Geothermal Field, Proceedings World Geothermal Congress.

Arnórsson, S. (1995), Geothermal systems in Iceland: Structure and conceptual models-I. High-temperature areas, Geothermics, 24(5-6), 561-602, doi:10.1016/03756505(95)00025-9.

Ásmundsson, R., P. Pezard, B. Sanjuan, J. Henninges, J.-L. Deltombe, N. Halladay, F. Lebert, A. Gadalia, R. Millot, B. Gibert, M. Violay, T. Reinsch, J.-M. Naisse, C. Massiot, P. Azas, D. Mainprice, C. Karytsas, and C. Johnston (2014), High temperature instruments and methods developed for supercritical geothermal reservoir characterisation and exploitation-The HiTI project, Geothermics, 49, 90-98, doi: 10.1016/j.geothermics.2013.07.008.

Aydin, A., and J. M. Degraff (1988), Evolution of Polygonal Fracture Patterns in Lava Flows, Science, 239(4839), 471-476.

Baghbanan, A., and L. Jing (2007), Hydraulic properties of fractured rock masses with correlated fracture length and aperture, International Journal of Rock Mechanics and Mining Sciences, 44(5), 704-719, doi:10.1016/j.ijrmms.2006.11.001.

Baghbanan, A., and L. Jing (2008), Stress effects on permeability in a fractured rock mass with correlated fracture length and aperture, International Journal of Rock Mechanics and Mining Sciences, 45(8), 1320-1334, doi:10.1016/j.ijrmms.2008.01. 015.

Bannister, S., S. Bourguignon, S. Sherburn, and T. Bertrand (2015), 3-D Seismic Velocity and Attenuation in the Central Taupo Volcanic Zone, New Zealand: Imaging the Roots of Geothermal Systems, Proceedings World Geothermal Congress.

Barth, N. C., V. G. Toy, R. M. Langridge, and R. J. Norris (2012), Scale dependence of oblique plate-boundary partitioning: New insights from LiDAR, central Alpine fault, New Zealand, Lithosphere, 4(5), 435-448, doi:10.1130/L201.1.

Barton, C. A., and M. D. Zoback (1992), Self-similar distribution and properties of macroscopic fractures at depth in crystalline rock in the Cajon Pass Scientific Drill Hole, Journal of Geophysical Research, 97(B4), 5181-5200, doi:10.1029/91jb01674. 
Barton, C. A., and M. D. Zoback (2002), Wellbore Imaging Technologies Applied to Reservoir Geomechanics and Environmental Engineering, in AAPG Methods in Exploration No. 13: Geological Applications of Well Logs, edited by M. Lovell and N. Parkinson, The American Association of Petroleum Geologists.

Barton, C. A., M. D. Zoback, and D. Moos (1995), Fluid flow along potentially active faults in crystalline rock, Geology, 23(8), 683-686, doi:10.1130/0091-7613(1995) 023〈0683:FFAPAF $\rangle$ 2.3.CO;2.

Barton, C. A., D. Moos, L. Hartley, S. Baxter, L. Foulquier, H. Holl, and R. Hogarth (2013), Geomechanically coupled simulation of flow in fractured reservoirs, Proceedings of the 38th workshop on geothermal engineering, Stanford.

Begg, J. G., and V. Mouslopoulou (2010), Analysis of late Holocene faulting within an active rift using lidar, Taupo Rift, New Zealand, Journal of Volcanology and Geothermal Research, 190(1-2), 152-167, doi:10.1016/j.jvolgeores.2009.06.001.

Benato, S., S. Hickman, N. C. Davatzes, J. Taron, P. Spielman, D. Elsworth, E. L. Majer, and K. Boyle (2016), Conceptual model and numerical analysis of the Desert Peak EGS project: Reservoir response to the shallow medium flow-rate hydraulic stimulation phase, Geothermics, 63, 139-156, doi:10.1016/j.geothermics.2015.06.008.

Berkowitz, B. (2002), Characterizing flow and transport in fractured geological media: A review, Advances in Water Resources, 25 (8-12), 861-884, doi:10.1016/s03091708(02)00042-8.

Berryman, K. R., U. A. Cochran, K. J. Clark, G. P. Biasi, R. M. Langridge, and P. Villamor (2012), Major Earthquakes Occur Regularly on an Isolated Plate Boundary Fault, Science, 336(6089), 1690-1694, doi:10.1126/science.1218959.

Bertani, R. (2016), Geothermal power generation in the world 2010-2014 update report, Geothermics, 60, 31-43, doi:10.1016/j.geothermics.2015.11.003.

Bertrand, E. A., T. G. Caldwell, S. Bannister, S. Soengkono, S. L. Bennie, G. J. Hill, and W. Heise (2015), Using array MT data to image the crustal resistivity structure of the southeastern Taupo Volcanic Zone, New Zealand, Journal of Volcanology and Geothermal Research, 305, 63-75, doi:10.1016/j.jvolgeores.2015.09.020.

Bibby, H., T. G. Caldwell, F. Davey, and T. Webb (1995), Geophysical evidence on the structure of the Taupo Volcanic Zone and its hydrothermal circulation, Journal of Volcanology and Geothermal Research, 68(1-3), 29-58, doi:10.1016/0377-0273(95) 00007-H.

Bignall, G. (2010), Hotter and Deeper: New Zealand's Research Programme to Harness its Deep Geothermal Resources, Proceedings World Geothermal Congress. 
Bignall, G., A. J. Rae, and M. Rosenberg (2010), Rationale for targeting fault versus formation-hosted permeability in high-temperature geothermal systems of the Taupo Volcanic Zone, New Zealand, Proceedings of the World Geothermal Congress.

Blake, K., and N. C. Davatzes (2012), Borehole image log and statistical analysis of FOH-3D, Fallon Naval Air Station, NV, Proceedings of the Thirty-Seventh Workshop on Geothermal Reservoir Engineering, Stanford.

Boese, C. M., J. Townend, E. Smith, and T. Stern (2012), Microseismicity and stress in the vicinity of the Alpine Fault, central Southern Alps, New Zealand, Journal of Geophysical Research: Solid Earth, 117(B02302), doi:10.1029/2011jb008460.

Boness, N. L., and M. D. Zoback (2006), A multiscale study of the mechanisms controlling shear velocity anisotropy in the San Andreas Fault Observatory at Depth, Geophysics, 71 (5), F131-F146, doi:10.1190/1.2231107.

Bonneau, F., V. Henrion, G. Caumon, P. Renard, and J. Sausse (2013), A methodology for pseudo-genetic stochastic modeling of discrete fracture networks, Computers $\&$ Geosciences, 56, 12-22, doi:10.1016/j.cageo.2013.02.004.

Bonnet, E., O. Bour, N. E. Odling, P. Davy, I. Main, P. Cowie, and B. Berkowitz (2001), Scaling of fracture systems in geological media, Reviews of Geophysics, 39(3), 347383, doi:10.1029/1999rg000074.

Bonnichsen, B., and D. F. Kauffman (1987), Physical features of rhyolite lava flows in the Snake River Plain volcanic province, southwestern Idaho, Geological Society of America Special Papers, 212, 119-145.

Botella, A., B. Lévy, and G. Caumon (2015), Indirect unstructured hex-dominant mesh generation using tetrahedra recombination, Computational Geosciences, 20(3), 437451, doi:10.1007/s10596-015-9484-9.

Boulton, C., B. M. Carpenter, V. Toy, and C. Marone (2012), Physical properties of surface outcrop cataclastic fault rocks, Alpine Fault, New Zealand, Geochemistry, Geophysics, Geosystems, 13(1), doi:10.1029/2011gc003872.

Bour, O., and P. Davy (1997), Connectivity of random fault networks following a power law fault length distribution, Water Resources, 33(7), 1567-1583, doi:10.1029/ 96WR00433.

Bour, O., and P. Davy (1998), On the connectivity of three-dimensional fault networks, Water Resources Research, 34(10), 2611-2622, doi:10.1029/98wr01861.

Bourguignon, S., S. Bannister, C. M. Henderson, J. Townend, and H. Zhang (2015), Structural heterogeneity of the midcrust adjacent to the central Alpine Fault, 
New Zealand: Inferences from seismic tomography and seismicity between Harihari and Ross, Geochemistry, Geophysics, Geosystems, 16(4), 1017-1043, doi: $10.1002 / 2014 \mathrm{gc} 005702$.

Brathwaite, R. L., H. J. Cargill, A. B. Christie, and A. Swain (2001), Lithological and spatial controls on the distribution of quartz veins in andesite- and rhyolite-hosted epithermal Au-Ag deposits of the Hauraki Goldfield, New Zealand, Mineralium Deposita, 36(1), 1-12, doi:10.1007/s001260050282.

Breede, K., K. Dzebisashvili, X. Liu, and G. Falcone (2013), A systematic review of enhanced (or engineered) geothermal systems: past, present and future, Geothermal Energy, 1(1), 4, doi:10.1186/2195-9706-1-4.

Browne, P., I. Graham, R. Parker, and C. Wood (1992), Subsurface andesite lavas and plutonic rocks in the Rotokawa and Ngatamariki geothermal systems, Taupo Volcanic Zone, New Zealand, Journal of Volcanology and Geothermal Research, 51(3), 199215, doi:10.1016/0377-0273(92)90123-u.

Brudy, M., and M. Zoback (1999), Drilling-induced tensile wall-fractures: implications for determination of in-situ stress orientation and magnitude, International Journal of Rock Mechanics and Mining Sciences, 36(2), 191-215, doi:10.1016/s0148-9062(98) 00182-x.

Buckley, S. J., J. Howell, H. Enge, and T. Kurz (2008), Terrestrial laser scanning in geology: data acquisition, processing and accuracy considerations, Journal of the Geological Society, 165(3), 625-638, doi:10.1144/0016-76492007-100.

Burnham, K. P., and D. R. Anderson (2002), Model selection and multimodel inference: a practical information-theoretic approach, 2nd ed., Springer, New York.

Burns, K. L. (1987), Geological Structures from Televiewer Logs of GT-2, Fenton Hill, New Mexico, Tech. rep., Los Alamos National Laboratory.

Caine, J., J. Evans, and C. Forster (1996), Fault zone architecture and permeability structure, Geology, 24(11), 1025-1028, doi:10.1130/0091-7613(1996)024<1025: FZAAPS $>2.3 . \mathrm{CO} ; 2$.

Carbotte, S. M., and K. C. Macdonald (1994), Comparison of seafloor tectonic fabric at intermediate, fast, and super fast spreading ridges: Influence of spreading rate, plate motions, and ridge segmentation on fault patterns, Journal of Geophysical Research: Solid Earth, 99 (B7), 13,609-13,631, doi:10.1029/93jb02971.

Carpenter, B., H. Kitajima, R. Sutherland, J. Townend, V. Toy, and D. Saffer (2014), Hydraulic and acoustic properties of the active Alpine Fault, New Zealand: Labora- 
tory measurements on DFDP-1 drill core, Earth and Planetary Science Letters, 390, 45-51, doi:10.1016/j.epsl.2013.12.023.

Chambefort, I., B. Lewis, C. Wilson, A. Rae, C. Coutts, G. Bignall, and T. Ireland (2014), Stratigraphy and structure of the Ngatamariki geothermal system from new zircon U-Pb geochronology: Implications for Taupo Volcanic Zone evolution, Journal of Volcanology and Geothermal Research, 274, 51-70, doi:10.1016/j.jvolgeores.2014. 01.015 .

Chen, R.-H., C.-H. Lee, and C.-S. Chen (2001), Evaluation of transport of radioactive contaminant in fractured rock, Environmental Geology, 41(3-4), 440-450, doi:10. $1007 / \mathrm{s} 002540100410$.

Chen, Z., S. Narayan, Z. Yang, and S. Rahman (2000), An experimental investigation of hydraulic behaviour of fractures and joints in granitic rock, International Journal of Rock Mechanics and Mining Sciences, 37(7), 1061-1071, doi:10.1016/s1365-1609(00) 00039-3.

Chester, F. M., J. P. Evans, and R. L. Biegel (1993), Internal structure and weakening mechanisms of the San Andreas Fault, Journal of Geophysical Research: Solid Earth, 98(B1), 771-786, doi:10.1029/92jb01866.

Childs, C., T. Manzocchi, J. J. Walsh, C. G. Bonson, A. Nicol, and M. P. Schpfer (2009), A geometric model of fault zone and fault rock thickness variations, Journal of Structural Geology, 31(2), 117-127, doi:10.1016/j.jsg.2008.08.009.

Chilès, J. P. (2005), Stochastic modeling of natural fractured media: a review, in Geostatistics Banff 2004, pp. 285-294, Springer.

Christie, A. B., M. P. Simpson, R. L. Brathwaite, J. L. Mauk, and S. F. Simmons (2007), Epithermal Au-Ag and Related Deposits of the Hauraki Goldfield, Coromandel Volcanic Zone, New Zealand, Economic Geology, 102(5), 785-816, doi: 10.2113/gsecongeo.102.5.785.

Clauset, A., C. R. Shalizi, and M. E. J. Newman (2009), Power-Law Distributions in Empirical Data, SIAM Rev., 51(4), 661-703, doi:10.1137/070710111.

CloudCompare (2016), version 2.6.2 (GPL software), http://cloudcompare.org.

Conin, M., S. Bourlange, P. Henry, A. Boiselet, and P. Gaillot (2014), Distribution of resistive and conductive structures in Nankai accretionary wedge reveals contrasting stress paths, Tectonophysics, 611, 181-191, doi:10.1016/j.tecto.2013.11.025.

Conway, C. E., D. B. Townsend, G. S. Leonard, C. J. N. Wilson, A. T. Calvert, and J. A. Gamble (2015), Lava-ice interaction on a large composite volcano: a case study 
from Ruapehu, New Zealand, Bulletin of Volcanology, 77(3), doi:10.1007/s00445015-0906-2.

Conway, C. E., G. S. Leonard, D. B. Townsend, A. T. Calvert, C. J. Wilson, J. A. Gamble, and S. R. Eaves (2016), A high-resolution 40Ar/39Ar lava chronology and edifice construction history for Ruapehu volcano, New Zealand, Journal of Volcanology and Geothermal Research, doi:10.1016/j.jvolgeores.2016.07.006.

Cox, S. C., and R. Sutherland (2007), Regional Geological Framework of South Island, New Zealand, and its Significance for Understanding the Active Plate Boundary, in A Continental Plate Boundary: Tectonics at South Island, New Zealand, edited by D. Okaya, T. Stern, and F. Davey, pp. 19-46, Wiley Online Library, doi:10.1029/ 175 GM03.

Cox, S. C., H. K. Rutter, A. Sims, M. Manga, J. J. Weir, T. Ezzy, P. A. White, T. W. Horton, and D. Scott (2012), Hydrological effects of the Mw 7.1 Darfield (Canterbury) earthquake, 4 September 2010, New Zealand, New Zealand Journal of Geology and Geophysics, 8306(55), doi:10.1080/00288306.2012.680474.

Cox, S. C., C. D. Menzies, R. Sutherland, P. H. Denys, C. Chamberlain, and D. A. H. Teagle (2015), Changes in hot spring temperature and hydrogeology of the Alpine Fault hanging wall, New Zealand, induced by distal South Island earthquakes, Geofluids, 15(1-2), 216-239, doi:10.1111/gfl.12093.

Cox, S. F. (1995), Faulting processes at high fluid pressures: an example of fault valve behavior from the Wattle Gully Fault, Victoria, Australia, Journal of Geophysical Research, 100(B7), 12,841-12,859, doi:10.1029/95JB00915.

Cox, S. F. (1999), Deformational controls on the dynamics of fluid flow in mesothermal gold systems, Geological Society, London, Special Publications, 155, 123-140, doi: 10.1144/GSL.SP.1999.155.01.10.

Cox, S. F. (2016), Injection-driven swarm seismicity and permeability enhancement: Implications for the dynamics of hydrothermal ore systems in high fluid-flux, overpressured faulting regimes - An invited paper, Economic Geology, 111 (3), 559-587, doi:10.2113/econgeo.111.3.559.

Craw, D. (1988), Shallow-level metamorphic fluids in a high uplift rat metamorphic belt; Alpine Schist, New Zealand, Journal of Metamorphic Geology, 6(1), 1-16, doi: 10.1111/j.1525-1314.1988.tb00405.x.

Craw, D. (1997), Fluid inclusion evidence for geothermal structure beneath the Southern Alps, New Zealand, New Zealand Journal of Geology and Geophysics, 40(1), 43-52, doi:10.1080/00288306.1997.9514739. 
Crider, J. G. (2015), The initiation of brittle faults in crystalline rock, Journal of Structural Geology, 77, 159-174, doi:10.1016/j.jsg.2015.05.001.

Cruden, D. (1977), Describing the size of discontinuities, International Journal of Rock Mechanics and Mining Sciences \& Geomechanics Abstracts, 14(3), 133-137, doi: 10.1016/0148-9062(77)90004-3.

Custodio, E. (2007), Groundwater in volcanic hard rocks, Groundwater in fractured rocks: IAH Selected Paper Series, 9, 95-108.

Daniel, J.-M., I. Moretti, L. Micarelli, S. E. Chuyne, and C. D. Piane (2004), Macroscopic structural analysis of AG10 well (Gulf of Corinth, Greece), Comptes Rendus Geoscience, 336(4-5), 435-444, doi:10.1016/j.crte.2004.01.001.

Darby, D. J., K. M. Hodgkinson, and G. H. Blick (2000), Geodetic measurement of deformation in the Taupo Volcanic Zone, New Zealand: The north Taupo network revisited, New Zealand Journal of Geology and Geophysics, 43(2), 157-170, doi: 10.1080/00288306.2000.9514878.

Davatzes, N. C., and S. H. Hickman (2010), Stress, fracture, and fluid-flow analysis using acoustic and electrical image logs in hot fractured granites of the Coso Geothermal Field, California, U.S.A., in Dipmeter and borehole image log technology: AAPG Memoir 92, edited by M. Poppelreiter, C. Garcia-Carballido, and M. Kraaijveld, pp. 259-293, AAPG Special Volumes, doi:10.1306/13181288M923134.

Davidson, J., and S. De Silva (2000), Composite volcanoes, in Encyclopedia of Volcanoes, edited by H. Sigurdsson, B. F. Houghton, H. Rymer, J. Stix, and S. McNutts, pp. 663-681, Academic Press.

Davidson, J., P. A. Siratovich, I. C. Wallis, D. M. Gravley, and D. D. McNamara (2012), Quantifying the stress distribution at the Rotokawa Geothermal Field, New Zealand, Proceedings of the 34th New Zealand Geothermal Workshop.

Davy, P., O. Bour, J. R. De Dreuzy, and C. Darcel (2006), Flow in multiscale fractal fracture networks, Geological Society, London, Special Publications, 261, 31-45, doi: 10.1144/GSL.SP.2006.261.01.03.

Davy, P., R. L. Goc, and C. Darcel (2013), A model of fracture nucleation, growth and arrest, and consequences for fracture density and scaling, Journal of Geophysical Research: Solid Earth, 118(4), 1393-1407, doi:10.1002/jgrb.50120.

de Dreuzy, J.-R., G. Pichot, B. Poirriez, and J. Erhel (2013), Synthetic benchmark for modeling flow in 3D fractured media, Computers $\&$ Geosciences, 50, 59-71, doi: 10.1016/j.cageo.2012.07.025. 
DeGraff, J. M., and A. Aydin (1987), Surface morphology of columnar joints and its significance to mechanics and direction of joint growth, Geological Society of America

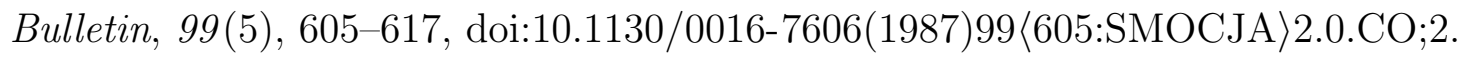

DeGraff, J. M., and A. Aydin (1993), Effect of thermal regime on growth increment and spacing of contraction joints in basaltic lava, Journal of Geophysical Research: Solid Earth, 98(B4), 6411-6430, doi:10.1029/92jb01709.

DeGraff, J. M., P. E. Long, and A. Aydin (1989), Use of joint-growth directions and rock textures to infer thermal regimes during solidification of basaltic lava flows, Journal of Volcanology and Geothermal Research, 38(3-4), 309-324, doi:10.1016/ 0377-0273(89)90045-0.

DeMets, C., R. G. Gordon, D. F. Argus, and S. Stein (1994), Effect of recent revisions to the geomagnetic reversal time scale on estimates of current plate motions, Geophysical research letters, 21 (20), 2191-2194, doi:10.1029/94GL02118.

Deng, B.-w., C.-h. Lee, and J.-l. Chang (1995), Characterization and interpretation of variability of fracture pattern in jointed andesites, Lan-Yu, Taiwan, Journal of the Chinese Institute of Engineers, 18(6), 787-799, doi:10.1080/02533839.1995.9677747.

Deparis, J., B. Fricout, D. Jongmans, T. Villemin, L. Effendiantz, and A. Mathy (2008), Combined use of geophysical methods and remote techniques for characterizing the fracture network of a potentially unstable cliff site (the 'Roche du Midi', Vercors massif, France), Journal of Geophysics and Engineering, 5(2), 147-157, doi:10.1088/ $1742-2132 / 5 / 2 / 002$.

Dershowitz, B., T. Eiben, S. Follin, and J. Andersson (1999), SR 97-Alternative models project. Discrete fracture network modelling for performance assessment of Aberg, Tech. rep., Swedish Nuclear Fuel and Waste Management Co.

Dershowitz, W. S., and H. H. Einstein (1988), Characterizing rock joint geometry with joint system models, Rock Mechanics and Rock Engineering, 21(21-51), 1988, doi:10.1007/BF01019674.

Dershowitz, W. S., and A. H. Herda (1992), Interpretation of fracture spacing and intensity, 33th U.S. Symposium on Rock Mechanics (USRMS).

Dewez, T. B., D. Girardeau-Montaut, C. Allanic, and J. Rohmer (2016), FACETS: a Cloudcompare plugin to extract geological planes from unstructured 3D point clouds, Int. Arch. Photogramm. Remote Sens. Spatial Inf. Sci., XLI-B5, 799-804, doi:10.5194/isprsarchives-xli-b5-799-2016. 
Dezayes, C., C. Lerouge, B. Sanjuan, C. Ramboz, and M. Brach (2015), Toward a Better Understanding of the Fluid Circulation in the Rhine Graben for a Better Geothermal Exploration of the Deep Basins, World Geothermal Congress 2015, p. 13.

Diederichs, M. S., P. K. Kaiser, and E. Eberhardt (2004), Damage initiation and propagation in hard rock during tunnelling and the influence of near-face stress rotation, International Journal of Rock Mechanics 85 Mining Sciences, 41(5), 785-812, doi: 10.1016/j.ijrmms.2004.02.003.

Doe, T., R. Mclaren, and W. S. Dershowitz (2014), Discrete Fracture Network Simulations of Enhanced Geothermal Systems, Proceedings of the Thirty-Ninth Workshop on Geothermal Reservoir Engineering.

Doyuran, V., C. Ayday, and N. Karahanoglu (1993), Statistical analyses of discontinuity parameters of Gölbaši (Ankara) Andesites, Süpren (Eskišehir) marble, and Porsuk Dam (Eskišehir) peridotite in Turkey, Bulletin of the International Association of Engineering Geology, 48(1), 15-31, doi:10.1007/bf02594973.

Eastwood, A. A., D. M. Gravley, C. J. N. Wilson, I. Chambefort, C. Oze, J. W. Cole, and T. R. Ireland (2013), U-PB dating of subsurface pyroclastic deposits (Tahorakuri Formation) at Ngatamariki and Rotokawa Geothermal Fields, Proceedings of the 35th New Zealand Geothermal Workshop, pp. 1-8.

Eberhardt, E., D. Stead, B. Stimpson, and R. S. Read (1998), Identifying crack initiation and propagation thresholds in brittle rock, Canadian Geotechnical Journal, 35(2), 222-233, doi:10.1139/t97-091.

Ellsworth, W. L. (2013), Injection-Induced Earthquakes, Science, 341 (6142), 1225,9421225,942, doi:10.1126/science.1225942.

Enge, H. D., S. J. Buckley, A. Rotevatn, and J. A. Howell (2007), From outcrop to reservoir simulation model: Workflow and procedures, Geosphere, 3(6), 469-490, doi:10.1130/ges00099.1.

Evans, K. F. (2005), Permeability creation and damage due to massive fluid injections into granite at $3.5 \mathrm{~km}$ at Soultz: 2. Critical stress and fracture strength, Journal of Geophysical Research, 110(B4), doi:10.1029/2004JB003169.

Evans, K. F., A. Genter, and J. Sausse (2005), Permeability creation and damage due to massive fluid injections into granite at $3.5 \mathrm{~km}$ at Soultz: 1 . Borehole observations, Journal of Geophysical Research: Solid Earth, 110(B4), doi:10.1029/2004jb003168.

Fabuel-Perez, I., D. Hodgetts, and J. Redfern (2010), Integration of digital outcrop models (DOMs) and high resolution sedimentology - workflow and implications for 
geological modelling: Oukaimeden Sandstone Formation, High Atlas (Morocco), Petroleum Geoscience, 16(2), 133-154, doi:10.1144/1354-079309-820.

Faulkner, D., C. Jackson, R. Lunn, R. Schlische, Z. Shipton, C. Wibberley, and M. Withjack (2010), A review of recent developments concerning the structure, mechanics and fluid flow properties of fault zones, Journal of Structural Geology, 32(11), 1557-1575, doi:10.1016/j.jsg.2010.06.009.

Feenstra, J., C. Thurber, J. Townend, S. Roecker, S. Bannister, C. Boese, N. Lord, S. Bourguignon, and D. Eberhart-Phillips (2016), Microseismicity and P-wave tomography of the central Alpine Fault, New Zealand, New Zealand Journal of Geology and Geophysics, pp. 1-13, doi:10.1080/00288306.2016.1182561.

Feng, Z.-Q. (2008), Volcanic rocks as prolific gas reservoir: A case study from the Qingshen gas field in the Songliao Basin, NE China, Marine and Petroleum Geology, 25 (4-5), 416-432, doi:10.1016/j.marpetgeo.2008.01.008.

Ferrero, A. M., G. Forlani, R. Roncella, and H. I. Voyat (2009), Advanced Geostructural Survey Methods Applied to Rock Mass Characterization, Rock Mechanics and Rock Engineering, 42(4), 631-665, doi:10.1007/s00603-008-0010-4.

Forbes, A., S. Blake, H. Tuffen, and A. Wilson (2014), Fractures in a trachyandesitic lava at Öræfajökull, Iceland, used to infer subglacial emplacement in 1727-8 eruption, Journal of Volcanology and Geothermal Research, 288, 8-18, doi:10.1016/j. jvolgeores.2014.10.004.

Fournier, R. (1998), Hydrothermal Processes Related to Movement of Fluid From Plastic into Brittle Rock in the Magmatic-Epithermal Environment, Economic Geology, 94 (8), 1193-1211, doi:10.2113/gsecongeo.94.8.1193.

Gamble, J. A., R. C. Price, I. E. M. Smith, W. C. Mcintosh, and N. W. Dunbar (2003), 40Ar/39Ar geochronology of magmatic activity, magma flux and hazards at Ruapehu volcano, Taupo Volcanic Zone, New Zealand, Journal of Volcanology and Geothermal Research, 120(3-4), 271-287, doi:10.1016/S0377-0273(02)00407-9.

García-Sellés, D., O. Falivene, P. Arbués, O. Gratacos, S. Tavani, and J. Muñoz (2011), Supervised identification and reconstruction of near-planar geological surfaces from terrestrial laser scanning, Computers \& Geosciences, 37(10), 1584-1594, doi:10.1016/j.cageo.2011.03.007.

Genter, A., and H. Traineau (1996), Analysis of macroscopic fractures in granite in the HDR geothermal well EPS-1, Soultz-sous-Forêts, France, Journal of Volcanology and Geothermal Research, 72(1-2), 121-141, doi:10.1016/0377-0273(95)00070-4. 
Genter, A., C. Castaing, C. Dezayes, H. Tenzer, H. Traineau, and T. Villemin (1997), Comparative analysis of direct (core) and indirect (borehole imaging tools) collection of fracture data in the Hot Dry Rock Soultz reservoir (France), Journal of Geophysical Research, 102(B7), 15,419-15,431, doi:10.1029/97JB00626.

Geyer, A., D. García-Sellés, D. Pedrazzi, S. Barde-Cabusson, J. Marti, and J. Muñoz (2015), Studying monogenetic volcanoes with a terrestrial laser scanner: case study at Croscat volcano (Garrotxa Volcanic Field, Spain), Bulletin of Volcanology, r7(22), doi:10.1007/s00445-015-0909-z.

Gigapan (2016), Gigapan, http://www.gigapan.com.

Gigli, G., and N. Casagli (2011), Semi-automatic extraction of rock mass structural data from high resolution LIDAR point clouds, International Journal of Rock Mechanics and Mining Sciences, 48(2), 187-198, doi:10.1016/j.ijrmms.2010.11.009.

Gillam, B. G., T. A. Little, E. Smith, and V. G. Toy (2013), Extensional shear band development on the outer margin of the Alpine mylonite zone, Tatare Stream, Southern Alps, New Zealand, Journal of Structural Geology, 54, 1-20, doi:10.1016/j.jsg. 2013.06.010.

Gillespie, P., C. Howard, J. Walsh, and J. Watterson (1993), Measurement and characterisation of spatial distributions of fractures, Tectonophysics, 226(1-4), 113-141, doi:10.1016/0040-1951(93)90114-y.

Gillespie, P. A., J. D. Johnston, M. A. Loriga, K. J. W. McCaffrey, J. J. Walsh, and J. Watterson (1999), Influence of layering on vein systematics in line samples, Geological Society, London, Special Publications, 155(1), 35-56, doi:10.1144/gsl.sp. 1999.155.01.05.

Grant, M. A. (2016), Physical performance indicators for HDR/EGS projects, Geothermics, 63, 2-4, doi:10.1016/j.geothermics.2015.01.004.

Grant, M. A., and P. F. Bixley (2011), Geothermal Reservoir Engineering, 2nd ed., 378 pp., Academic Press, Burlington, USA, doi:10.1016/B978-0-12-383880-3.10022-8.

Grossenbacher, K. A., and S. M. McDuffie (1995), Conductive cooling of lava: columnar joint diameter and stria width as functions of cooling rate and thermal gradient, Journal of Volcanology and Geothermal Research, 69 (1-2), 95-103, doi:10.1016/03770273(95)00032-1.

Gudmundsson, A. (2000), Fracture dimensions, displacements and fluid transport, Journal of Structural Geology, 22(9), 1221-1231, doi:10.1016/S0191-8141(00)000523. 
Gudmundsson, A. (2011), Deflection of dykes into sills at discontinuities and magmachamber formation, Tectonophysics, 500(1-4), 50-64, doi:10.1016/j.tecto.2009.10. 015 .

Gudmundsson, A., S. S. Berg, K. B. Lyslo, and E. Skurtveit (2001), Fracture networks and fluid transport in active fault zones, Journal of Structural Geology, 23(2-3), 343-353, doi:10.1016/s0191-8141(00)00100-0.

Gudmundsson, A., I. Fjeldskaar, and S. L. Brenner (2002), Propagation pathways and fluid transport of hydrofractures in jointed and layered rocks in geothermal fields, Journal of Volcanology and Geothermal Research, 116(3-4), 257-278, doi: 10.1016/S0377-0273(02)00225-1.

Guéguen, Y., and V. Palciauskas (1994), Introduction to the physics of rocks, Princeton University Press.

Guerriero, V., S. Vitale, S. Ciarcia, and S. Mazzoli (2011), Improved statistical multiscale analysis of fractured reservoir analogues, Tectonophysics, 504 (1-4), 14-24, doi: 10.1016/j.tecto.2011.01.003.

Hackett, W. R., and B. F. Houghton (1989), A facies model for a quaternary andesitic composite volcano: Ruapehu, New Zealand, Bulletin of Volcanology, 51(1), 51-68, doi:10.1007/bf01086761.

Halwa, L., I. C. Wallis, and G. T. Lozada (2013), Geological analysis of the volcanic subsurface using borehole reistibity images in the Ngatamariki Geothermal Field, New Zealand, 35th New Zealand Geothermal Workshop.

Hammah, R., and J. Curran (1998), Fuzzy cluster algorithm for the automatic identification of joint sets, International Journal of Rock Mechanics and Mining Sciences, 35(7), 889-905, doi:10.1016/s0148-9062(98)00011-4.

Hammah, R. E., and J. H. Curran (1999), On Distance Measures for the Fuzzy K-means Algorithm for Joint Data, Rock Mechanics and Rock Engineering, 32(1), 1-27, doi: $10.1007 / \mathrm{s} 006030050041$.

Hansen, B., and J. Buczak (2010), Making interpretable images from image logs, in Dipmeter and borehole image log technology: AAPG Memoir 92, edited by M. Poppelreiter, C. Garcia-Carballido, and M. Kraaijveld, pp. 51-66, AAPG, doi: 10.1306/13181277M923405.

Hanson, C. R., R. J. Norris, and A. F. Cooper (1990), Regional fracture patterns east of the Alpine Fault between the Fox and Franz Josef Glaciers, Westland, New Zealand, New Zealand Journal of Geology and Geophysics, 33(4), 617-622, doi:10. 1080/00288306.1990.10421379. 
Hardebol, N., and G. Bertotti (2013), DigiFract: A software and data model implementation for flexible acquisition and processing of fracture data from outcrops, Computers \& Geosciences, 54, 326-336, doi:10.1016/j.cageo.2012.10.021.

Heap, M., Y. Lavallée, L. Petrakova, P. Baud, T. Reuschle, N. Varley, and D. B. Dingwell (2014), Microstructural controls on the physical and mechanical properties of edifice-forming andesites at Volcán de Colima, Mexico, Journal of Geophysical Research: Solid Earth, 119(4), 2925-2963, doi:10.1002/2013JB010521.

Heap, M. J., and B. M. Kennedy (2016), Exploring the scale-dependent permeability of fractured andesite, Earth and Planetary Science Letters, 447, 139-150, doi:10.1016/ j.epsl.2016.05.004.

Hernandez, D., J. Clearwater, J. Burnell, P. Franz, L. Azwar, and A. Marsh (2015), Update on the Modeling of the Rotokawa Geothermal System: 2010-2014, Proceedings of the World Geothermal Congress.

Hetényi, G., B. Taisne, F. Garel, É. Médard, S. Bosshard, and H. B. Mattsson (2011), Scales of columnar jointing in igneous rocks: field measurements and controlling factors, Bulletin of Volcanology, 74 (2), 457-482, doi:10.1007/s00445-011-0534-4.

Hickman, S., and M. Zoback (2004), Stress orientations and magnitudes in the SAFOD pilot hole, Geophysical Research Letters, 31(15), doi:10.1029/2004gl020043.

Hickman, S., R. Sibson, and R. Bruhn (1995), Introduction to special section: Mechanical involvement of fluids in faulting, Journal of Geophysical Research, 100 (B7), 12,831-12,840, doi:10.1029/95JB01121.

Hobden, B. J., B. F. Houghton, M. A. Lanphere, and I. A. Nairn (1996), Growth of the Tongariro volcanic complex: New evidence from K-Ar age determinations, New Zealand Journal of Geology and Geophysics, 39(1), 151-154, doi:10.1080/00288306. 1996.9514701.

Hodgetts, D. (2013), Laser scanning and digital outcrop geology in the petroleum industry: A review, Marine and Petroleum Geology, 46, 335-354, doi:10.1016/j. marpetgeo.2013.02.014.

Hofrichter, J., and G. Winkler (2006), Statistical analysis for the hydrogeological evaluation of the fracture networks in hard rocks, Environmental Geology, 49(6), 821-827, doi:10.1007/s00254-006-0177-5.

Holm, D., R. Norris, and D. Craw (1989), Brittle and ductile deformation in a zone of rapid uplift: Central Southern Alps, New Zealand, Tectonics, 8(2), 153-168, doi: 10.1029/TC008i002p00153. 
Hopp, C., M. Savage, J. Townend, S. Sewell, and S. Sherburn (2016), Matched filter earthquake detection and double-difference relocation at Rotokawa and Ngatamariki geothermal areas: January to November, 2015, Proceedings 38th New Zealand Geothermal Workshop.

Houghton, B. F., J. H. Latter, and W. R. Hackett (1987), Volcanic hazard assessment for Ruapehu composite volcano, Taupo Volcanic Zone, New Zealand, Bulletin of Volcanology, 49, 737-751, doi:10.1007/BF01079825.

Hudson, J., and S. Priest (1979), Discontinuities and rock mass geometry, International Journal of Rock Mechanics and Mining Sciences $\&$ Geomechanics Abstracts, 16(6), 339-362, doi:10.1016/0148-9062(79)90001-9.

Hudson, J., and S. Priest (1983), Discontinuity frequency in rock masses, International Journal of Rock Mechanics and Mining Sciences 86 Geomechanics Abstracts, 20(2), 73-89, doi:10.1016/0148-9062(83)90329-7.

Hung, J.-H., K.-F. Ma, C.-Y. Wang, H. Ito, W. Lin, and E.-C. Yeh (2009), Subsurface structure, physical properties, fault-zone characteristics and stress state in scientific drill holes of Taiwan Chelungpu Fault Drilling Project, Tectonophysics, 466(3-4), 307-321, doi:10.1016/j.tecto.2007.11.014.

Ikari, M. J., B. M. Carpenter, A. J. Kopf, and C. Marone (2014), Frictional strength, rate-dependence, and healing in DFDP-1 borehole samples from the Alpine Fault, New Zealand, Tectonophysics, 630(C), 1-8, doi:10.1016/j.tecto.2014.05.005.

Ikari, M. J., S. Trütner, B. M. Carpenter, and A. J. Kopf (2015), Shear behavior of DFDP-1 borehole samples from the Alpine Fault, New Zealand, under a wide range of experimental conditions, International Journal of Earth Sciences, 104(6), 1523-1535, doi:10.1007/s00531-014-1115-5.

Ishibashi, T., N. Watanabe, N. Hirano, A. Okamoto, and N. Tsuchiya (2014), Beyondlaboratory-scale prediction for channeling flows through subsurface rock fractures with heterogeneous aperture distributions revealed by laboratory evaluation, Journal of Geophysical Research: Solid Earth, 120(1), 106-124, doi:10.1002/2014JB011555.

Ishibashi, T., N. Watanabe, H. Asanuma, and N. Tsuchiya (2016), Linking microearthquakes to fracture permeability change: The role of surface roughness, Geophysical Research Letters, 43(14), 7486-7493, doi:10.1002/2016gl069478.

Ito, H., and T. Kiguchi (2005), Distribution and properties of fractures in and around the Nojima Fault in the Hirabayashi GSJ borehole, in Petrophysical Properties of Crystalline Rocks, vol. 240, pp. 61-74, Geological Society, London, Special Publications, doi:10.1144/GSL.SP.2005.240.01.06. 
James, G., D. Witten, T. Hastie, and R. Tibshirani (2013), An Introduction to Statistical Learning, Springer New York, doi:10.1007/978-1-4614-7138-7.

James, M. R., H. Pinkerton, and L. J. Applegarth (2009), Detecting the development of active lava flow fields with a very-long-range terrestrial laser scanner and thermal imagery, Geophysical Research Letters, 36(22), doi:10.1029/2009gl040701.

Jeppson, T. N., and H. J. Tobin (2015), San Andreas fault zone velocity structure at SAFOD at core, log, and seismic scales, Journal of Geophysical Research: Solid Earth, 120(7), 4983-4997, doi:10.1002/2015jb012043.

Johnston, J. D., and K. J. W. McCaffrey (1996), Fractal geometries of vein systems and the variation of scaling relationships with mechanism, Journal of Structual Geology, 18(2-3), doi:10.1016/S0191-8141(96)80055-1.

Johri, M., E. M. Dunham, M. D. Zoback, and Z. Fang (2014), Predicting fault damage zones by modeling dynamic rupture propagation and comparison with field observations, Journal of Geophysical Research: Solid Earth, 119(2), 1251-1272, doi: 10.1002/2013jb010335.

Jones, R. R., K. J. W. Mccaffrey, J. Imber, R. Wightman, S. A. F. Smith, R. E. Holdsworth, P. Clegg, N. D. Paola, D. Healy, and R. W. Wilson (2008), Calibration and validation of reservoir models: the importance of high resolution, quantitative outcrop analogues, Geological Society, London, Special Publications, 309(1), 87-98, doi:10.1144/sp309.7.

Julio, C., G. Caumon, and M. Ford (2015), Sampling the uncertainty associated with segmented normal fault interpretation using a stochastic downscaling method, Tectonophysics, 639, 56-67, doi:10.1016/j.tecto.2014.11.013.

Kamb, W. B. (1959), Ice Petrofabric Observations from Blue Glacier, Washington, in Relation to Theory and Experiment, Journal of Geophysical Research, 64(11), doi:10.1029/JZ064i011p01891.

Karpuz, C., and A. Paşamehmetoğlu (1997), Field characterisation of weathered Ankara andesites, Engineering Geology, 46(1), 1-17, doi:10.1016/s0013-7952(96) 00002-6.

Kattenhorn, S. A., and C. J. Schaefer (2008), Thermal-mechanical modeling of cooling history and fracture development in inflationary basalt lava flows, Journal of Volcanology and Geothermal Research, 170(3-4), 181-197, doi:10.1016/j.jvolgeores. 2007.10.002. 
Kissling, W., A. Rae, P. Villamor, and S. Ellis (2015a), Hydrothermal Fluid Flow in a Structurally-controlled Basin, Ngakuru Graben, Taupo Rift, New Zealand, World Geothermal Congress 2015, p. 11.

Kissling, W. M., S. E. Ellis, D. D. McNamara, and C. Massiot (2015b), Modelling Fluid Flow Through Fractured Rock: Examples Using TVZ Geothermal Reservoirs, Proceedings 37th New Zealand Geothermal Workshop.

Koca, M. Y., C. Kincal, A. T. Arslan, and H. R. Yilmaz (2011), Anchor application in Karatepe andesite rock slope, Izmir-Türkiye, International Journal of Rock Mechanics and Mining Sciences, 48(2), 245-258, doi:10.1016/j.ijrmms.2010.11.006.

Krupp, R. E., and T. M. Seward (1987), The Rotokawa geothermal system, New Zealand: an active epithermal gold-depositing environment, Economic Geology, 82(5), 1109-1129, doi:10.2113/gsecongeo.82.5.1109.

Kulander, B. R., S. L. Dean, and B. J. Ward (1990), Fractured core analysis: Interpretation, logging, and use of natural and induced fractures in core, in AAPG Methods in Exploration Series 8: Tulsa, Oklahoma, p. 88, AAPG.

Kulatilake, P., D. Wathugala, and O. Stephansson (1993), Joint network modelling with a validation exercise in Stripa mine, Sweden, International Journal of Rock Mechanics and Mining Sciences $\&$ Geomechanics Abstracts, 30(5), 503-526, doi: 10.1016/0148-9062(93)92217-e.

Lachassagne, P., B. Aunay, N. Frissant, M. Guilbert, and A. Malard (2014), Highresolution conceptual hydrogeological model of complex basaltic volcanic islands: a Mayotte, Comoros, case study, Terra Nova, 26(4), 307-321, doi:10.1111/ter.12102.

Ladeira, F., and N. Price (1981), Relationship between fracture spacing and bed thickness, Journal of Structural Geology, 3(2), 179-183, doi:10.1016/0191-8141(81)900134 .

Lahsen, A., J. Rojas, D. Morata, and D. Aravena (2015), Geothermal Exploration in Chile: Country Update, World Geothermal Congress 2015.

Laslett, G. M. (1982), Censoring and edge effects in areal and line transect sampling of rock joint traces, Journal of the International Association for Mathematical Geology, 14 (2), 125-140, doi:10.1007/bf01083948.

Lato, M. J., M. S. Diederichs, and D. J. Hutchinson (2010), Bias Correction for Viewlimited Lidar Scanning of Rock Outcrops for Structural Characterization, Rock Mechanics and Rock Engineering, 43(5), 615-628, doi:10.1007/s00603-010-0086-5. 
Laubach, S. E., J. E. Olson, and M. R. Gross (2009), Mechanical and fracture stratigraphy, AAPG Bulletin, 93(11), 1413-1426, doi:10.1306/07270909094.

Lee, C.-C., C.-H. Lee, H.-F. Yeh, and H.-I. Lin (2010), Modeling spatial fracture intensity as a control on flow in fractured rock, Environmental Earth Sciences, 63(6), 1199-1211, doi:10.1007/s12665-010-0794-x.

Lee, S. H., M. F. Lough, and C. L. Jensen (2001), Hierarchical modeling of flow in naturally fractured formations with multiple length scales, Water Resources Research, 37(3), 443-455, doi:10.1029/2000WR900340.

Lescinsky, T., and H. Fink (2000), Lava and ice interaction at stratovolcanoes: Use of characteristic features to determine past glacial extents and future volcanic hazards, Journal of Geophysical Research: Solid Earth, 105(B10), 23,711-23,726, doi:10.1029/ 2000JB900214.

Li, H., H. Wang, Z. Xu, J. Si, J. Pei, T. Li, Y. Huang, S.-R. Song, L.-W. Kuo, Z. Sun, M.-L. Chevalier, and D. Liu (2013), Characteristics of the fault-related rocks, fault zones and the principal slip zone in the Wenchuan Earthquake Fault Scientific Drilling Project Hole-1 (WFSD-1), Tectonophysics, 584, 23-42, doi:10.1016/j.tecto. 2012.08.021.

Li, H., Z. Xu, Y. Niu, G. Kong, Y. Huang, H. Wang, J. Si, Z. Sun, J. Pei, Z. Gong, M.L. Chevalier, and D. Liu (2014a), Structural and physical property characterization in the Wenchuan earthquake Fault Scientific Drilling project - hole 1 (WFSD-1), Tectonophysics, 619-620, 86-100, doi:10.1016/j.tecto.2013.08.022.

Li, Y., Q. Wang, J. Chen, L. Xu, and S. Song (2014b), K-means Algorithm Based on Particle Swarm Optimization for the Identification of Rock Discontinuity Sets, Rock Mechanics and Rock Engineering, 48, 375-385, doi:10.1007/s00603-014-0569-x.

Limpert, E., W. A. Stahel, and M. Abbt (2001), Log-normal Distributions across the Sciences: Keys and Clues, BioScience, 51 (5), 341-352, doi:10.1641/0006-3568(2001) 051[0341:Indats] 2.0.co;2.

Lin, A., T. Maruyama, and K. Kobayashi (2007), Tectonic implications of damage zone-related fault-fracture networks revealed in drill core through the Nojima fault, Japan, Tectonophysics, 443(3-4), 161-173, doi:10.1016/j.tecto.2007.01.011.

Lin, W., M. Conin, J. C. Moore, F. M. Chester, Y. Nakamura, J. J. Mori, L. Anderson, E. E. Brodsky, N. Eguchi, B. Cook, T. Jeppson, M. Wolfson-Schwehr, Y. Sanada, S. Saito, Y. Kido, T. Hirose, J. H. Behrmann, M. Ikari, K. Ujiie, C. Rowe, J. Kirkpatrick, S. Bose, C. Regalla, F. Remitti, V. Toy, P. Fulton, T. Mishima, T. Yang, T. Sun, T. Ishikawa, J. Sample, K. Takai, J. Kameda, S. Toczko, L. Maeda, 
S. Kodaira, R. Hino, and D. Saffer (2013), Stress State in the Largest Displacement Area of the 2011 Tohoku-Oki Earthquake, Science, 339(6120), 687-690, doi: 10.1126/science.1229379.

Litchfield, N., R. V. Dissen, R. Sutherland, P. Barnes, S. Cox, R. Norris, R. Beavan, R. Langridge, P. Villamor, K. Berryman, M. Stirling, A. Nicol, S. Nodder, G. Lamarche, D. Barrell, J. Pettinga, T. Little, N. Pondard, J. Mountjoy, and K. Clark (2014), A model of active faulting in New Zealand, New Zealand Journal of Geology and Geophysics, 57(1), 32-56, doi:10.1080/00288306.2013.854256.

Little, T., R. Holcombe, and B. Ilg (2002), Ductile fabrics in the zone of active oblique convergence near the Alpine Fault, New Zealand: identifying the neotectonic overprint, Journal of Structural Geology, 24(1), 193-217, doi:10.1016/s01918141(01)00059-1.

Little, T. A., S. Cox, J. K. Vry, and G. Batt (2005), Variations in exhumation level and uplift rate along the obliqu-slip Alpine fault, central Southern Alps, New Zealand, Geological Society of America Bulletin, 117(5-6), 707-723, doi:10.1130/b25500.1.

Little, T. A., D. J. Prior, and V. G. Toy (2016), Are quartz LPOs predictably oriented with respect to the shear zone boundary?: A test from the Alpine Fault mylonites, New Zealand, Geochemistry, Geophysics, Geosystems, 17(3), 981-999, doi:10.1002/ $2015 \mathrm{gc006145.}$

Lofts, J. C., and L. T. Bourke (1999), The recognition of artefacts from acoustic and resistivity borehole imaging devices, Geological Society, London, Special Publications, 159, 59-76, doi:10.1144/gsl.sp.1999.159.01.03.

Lore, J., H. Gao, and A. Aydin (2000), Viscoelastic thermal stress in cooling basalt flows, Journal of Geophysical Research: Solid Earth, 105 (B10), 23,695-23,709, doi: 10.1029/2000JB900226.

Lore, J., A. Aydin, and K. Goodson (2001), A deterministic methodology for prediction of fracture distribution in basaltic multiflows, Journal of Geophysical Research: Solid Earth, 106(B4), 6447-6459, doi:10.1029/2000JB900407.

Lund, J. W., and T. L. Boyd (2016), Direct utilization of geothermal energy 2015 worldwide review, Geothermics, 60, 66-93, doi:10.1016/j.geothermics.2015.11.004.

Manzocchi, T., C. Childs, and J. J. Walsh (2010), Faults and fault properties in hydrocarbon flow models, Geofluids, 10(1-2), 94-113, doi:10.1111/j.1468-8123.2010.00283. $\mathrm{x}$. 
Masoudi, P., B. Tokhmechi, M. Jafari, and B. Moshiri (2012), Application of fuzzy classifier fusion in determining productive zones in oil wells, Energy, Exploration \&6 Exploitation, 30(3), 403-415, doi:10.1260/0144-5987.30.3.403.

Massey, C. I., V. Manville, G. H. Hancox, H. J. Keys, C. Lawrence, and M. McSaveney (2010), Out-burst flood (lahar) triggered by retrogressive landsliding, 18 March 2007 at Mt Ruapehu, New Zealand - a successful early warning, Landslides, 7(3), 303-315, doi:10.1007/s10346-009-0180-5.

Massiot, C., G. Bignall, S. Alcaraz, A. J. Rae, F. Sepulveda, and H. van Moerkerk (2011), Testing the Effectiveness of Leapfrog Geothermal 3D Integrated Geological Modelling as a Geothermal Resource Exploration and Management Tool, GRC transactions, 35, 905-909.

Massiot, C., D. McNamara, and B. Lewis (2015), Processing and analysis of high temperature geothermal acoustic borehole image logs in the Taupo Volcanic Zone, New Zealand, Geothermics, 53, 190-201, doi:10.1016/j.geothermics.2014.05.010.

Matter, J. M., M. Stute, S. O. Snæbjörnsdottir, E. H. Oelkers, S. R. Gislason, E. Aradottir, B. Sigfusson, I. Gunnarsson, H. Sigurdardottir, E. Gunnlaugsson, G. Axelsson, H. A. Alfredsson, D. Wolff-Boenisch, K. Mesfin, D. Fernandez de la Reguera Taya, J. Hall, K. Dideriksen, and W. S. Broecker (2016), Rapid carbon mineralization for permanent disposal of anthropogenic carbon dioxide emissions, Science, 352(6291), 1312-1314, doi:10.1126/science.aad8132.

Mauldon, M., W. Dunne, and M. Rohrbaugh (2001), Circular scanlines and circular windows: new tools for characterizing the geometry of fracture traces, Journal of Structural Geology, 23(2-3), 247-258, doi:10.1016/s0191-8141(00)00094-8.

Mazzarini, F., M. Favalli, I. Isola, M. Neri, and M. T. Pareschi (2008), Surface roughness of pyroclastic deposits at Mt. Etna by 3D laser scanning, Annals of Geophysics, 51(5-6), doi:10.4401/ag-4461.

McCaffrey, K. J. W., J. M. Sleight, S. Pugliese, and R. E. Holdsworth (2003), Fracture formation and evolution in crystalline rocks: Insights from attribute analysis, Geological Society, London, Special Publications, 214(1), 109-124, doi: 10.1144/gsl.sp.2003.214.01.07.

Mclean, K., and D. D. McNamara (2011), Fractures interpreted from acoustic formation imaging technology: correlation to permeability, Proceedings of the Thirty-Sixth Workshop on Geothermal Reservoir Engineering.

McNamara, D. D., C. Massiot, B. Lewis, and I. C. Wallis (2015), Heterogeneity of structure and stress in the Rotokawa Geothermal Field, New Zealand, Journal of Geophysical Research: Solid Earth, 120(2), 1243-1262, doi:10.1002/2014JB011480. 
McNamara, D. D., S. Sewell, E. Buscarlet, and I. C. Wallis (2016a), A review of the Rotokawa Geothermal Field, New Zealand, Geothermics, 59(B), 281-293, doi: 10.1016/j.geothermics.2015.07.007.

McNamara, D. D., A. Lister, and D. J. Prior (2016b), Calcite sealing in a fractured geothermal reservoir: Insights from combined EBSD and chemistry mapping, Journal of Volcanology and Geothermal Research, 323, 38-52, doi:10.1016/j.jvolgeores.2016. 04.042 .

Menzies, C. D., D. A. H. Teagle, D. Craw, S. Cox, A. J. Boyce, C. D. Barrie, and S. Roberts (2014), Incursion of meteoric waters into the ductile regime in an active orogen, Earth and Planetary Science Letters, 399, 1-13, doi:10.1016/j.epsl.2014.04. 046.

Milicich, S. D., J. Clark, C. Wong, and M. Askari (2016), A review of the Kawerau Geothermal Field, New Zealand, Geothermics, 59(B), 252-265, doi:10.1016/j. geothermics.2015.06.012.

Milloy, S. F., and Y. W. Lim (2012), Wairakei-Tauhara pressure regime update, Proceedings of the New Zealand Geothermal Workshop.

Milloy, S. F., K. Mclean, and D. D. McNamara (2015), Comparing Borehole Televiewer Logs with Continuous Core: An Example from New Zealand, World Geothermal Congress Proceedings.

Ministry of Business Innovation and Employment (2016), Energy in New Zealand, 58 pp.

Misra, S., S. Ellis, and N. Mandal (2015), Fault damage zones in mechanically layered rocks: The effects of planar anisotropy, Journal of Geophysical Research: Solid Earth, 120(8), 5432-5452, doi:10.1002/2014jb011780.

Mitchell, T. M., and D. R. Faulkner (2008), Experimental measurements of permeability evolution during triaxial compression of initially intact crystalline rocks and implications for fluid flow in fault zones, Journal of Geophysical Research: Solid Earth, 113(B11412), 1-16, doi:10.1029/2008JB005588.

Mongillo, M. A., and L. Clelland (1984), Concise listing of information on the thermal areas and thermal springs of New Zealand, Tech. rep., Geothermal Coordination Group, DSIR, Wellington, New Zealand.

Mroczek, E. K., S. D. Milicich, P. F. Bixley, F. Sepulveda, E. A. Bertrand, S. Soengkono, and A. J. Rae (2016), Ohaaki geothermal system: Refinement of a conceptual reservoir model, Geothermics, 59(B), 311-324, doi:10.1016/j.geothermics.2015. 09.002 . 
Nelson, C. E., D. A. Jerram, R. W. Hobbs, R. Terrington, and H. Kessler (2011), Reconstructing flood basalt lava flows in three dimensions using terrestrial laser scanning, Geosphere, 7(1), 87-96, doi:10.1130/ges00582.1.

Nelson, C. E., R. W. Hobbs, and R. Rusch (2015), On the Use of Fractal Surfaces to Understand Seismic Wave Propagation in Layered Basalt Sequences, Pure and Applied Geophysics, 172(7), 1879-1892, doi:10.1007/s00024-014-0986-5.

Nemčok, M., J. N. Moore, R. Allis, and J. McCulloch (2004), Fracture development within a stratovolcano: the Karaha-Telaga Bodas geothermal field, Java volcanic arc, Geological Society, London, Special Publications, 231(1), 223-242, doi:10.1144/ gsl.sp.2004.231.01.13.

Nemčok, M., J. N. Moore, C. Christensen, R. Allis, T. Powell, B. Murray, and G. Nash (2007), Controls on the Karaha-Telaga Bodas geothermal reservoir, Indonesia, Geothermics, 36(1), 9-46, doi:10.1016/j.geothermics.2006.09.005.

Neuman, S. P. (2005), Trends, prospects and challenges in quantifying flow and transport through fractured rocks, Hydrogeology Journal, 13(1), 124-147, doi: 10.1007/s10040-004-0397-2.

Newman, M. (2005), Power laws, Pareto distributions and Zipf's law, Contemporary Physics, 46(5), 323-351, doi:10.1080/00107510500052444.

Ni, N., N. Chen, J. Chen, and M. Liu (2016), Integrating WorldView-2 imagery and terrestrial LiDAR point clouds to extract dyke swarm geometry: Implications for magma emplacement mechanisms, Journal of Volcanology and Geothermal Research, 310, 1-11, doi:10.1016/j.jvolgeores.2015.11.020.

Nicol, A., J. Walsh, J. Watterson, and P. Gillespie (1996), Fault size distributions are they really power-law?, Journal of Structural Geology, 18(2-3), 191-197, doi: 10.1016/s0191-8141(96)80044-7.

Nicol, A., J. Walsh, K. Berryman, and P. Villamor (2006), Interdependence of fault displacement rates and paleoearthquakes in an active rift, Geology, 34(10), 865, doi:10.1130/g22335.1.

Nicol, A., C. Mazengarb, F. Chanier, G. Rait, C. Uruski, and L. Wallace (2007), Tectonic evolution of the active Hikurangi subduction margin, New Zealand, since the Oligocene, Tectonics, 26(4), doi:10.1029/2006TC002090.

Nicol, A., R. Robinson, R. V. Dissen, and A. Harvison (2016), Variability of recurrence interval and single-event slip for surface-rupturing earthquakes in New Zealand, New Zealand Journal of Geology and Geophysics, 59(1), 97-116, doi:10.1080/00288306. 2015.1127822 . 
Nie, X., C. Zou, L. Pan, Z. Huang, and D. Liu (2013), Fracture analysis and determination of in-situ stress direction from resistivity and acoustic image logs and core data in the Wenchuan Earthquake Fault Scientific Drilling Borehole-2 (50-1370m), Tectonophysics, 593, 161-171, doi:10.1016/j.tecto.2013.03.005.

Norris, R., P. Koons, and A. Cooper (1990), The obliquely-convergent plate boundary in the South Island of New Zealand: implications for ancient collision zones, Journal of Structural Geology, 12(5-6), 715-725, doi:10.1016/0191-8141(90)90084-c.

Norris, R. J., and A. F. Cooper (1995), Origin of small-scale segmentation and transpressional thrusting along the Alpine fault, New Zealand, GSA Bulletin, 107(2), 231-240, doi:10.1130/0016-7606(1995)107〈0231:OOSSSA〉2.3.CO;2.

Norris, R. J., and A. F. Cooper (1997), Erosional control on the structural evolution of a transpressional thrust complex on the Alpine fault, New Zealand, Journal of Structural Geology, 19(10), 1323-1342, doi:10.1016/s0191-8141(97)00036-9.

Norris, R. J., and A. F. Cooper (2001), Late Quaternary slip rates and slip partitioning on the Alpine Fault, New Zealand, Journal of Structural Geology, 23(2-3), 507-520, doi:10.1016/S0191-8141(00)00122-X.

Norris, R. J., and A. F. Cooper (2003), Very high strains recorded in mylonites along the Alpine Fault, New Zealand: implications for the deep structure of plate boundary faults, Journal of Structural Geology, 25(12), 2141-2157, doi:10.1016/s0191-8141(03) 00045-2.

Norris, R. J., and A. F. Cooper (2007), The Alpine Fault, New Zealand: Surface Geology and Field Relationships, in A Continental Plate Boundary: Tectonics at South Island, New Zealand, edited by T. S. D. Okaya and F. Davey, pp. 157-175, American Geophysical Union, Washington, D. C., doi:10.1029/175GM09.

Odling, N. E., P. Gillespie, B. Bourgine, C. Castaing, J. P. Chiles, N. P. Christensen, E. Fillion, A. Genter, C. Olsen, L. Thrane, R. Trice, E. Aarseth, J. J. Walsh, and J. Watterson (1999), Variations in fracture system geometry and their implications for fluid flow in fractures hydrocarbon reservoirs, Petroleum Geoscience, 5(4), 373384, doi:10.1144/petgeo.5.4.373.

Olariu, M. I., J. F. Ferguson, C. L. Aiken, and X. Xu (2008), Outcrop fracture characterization using terrestrial laser scanners: Deep-water Jackfork sandstone at Big Rock Quarry, Arkansas, Geosphere, 4(1), 247, doi:10.1130/ges00139.1.

Özkaya, S. I. (2003), Fracture Length Estimation from Borehole Image Logs, Mathematical Geology, 35(6), 737-753, doi:10.1023/b:matg.0000002987.69549.ba. 
Paillet, F., and K. Kapucu (1989), Fracture characterization and fracture-permeability estimates from geophysical logs in the Mirror Lake watershed, New Hampshire, Tech. rep., U.S.G.S., Report 89-4058.

Pearce, M. A., R. R. Jones, S. A. F. Smith, and K. J. W. McCaffrey (2011), Quantification of fold curvature and fracturing using terrestrial laser scanning, AAPG Bulletin, 95(5), 771-794, doi:10.1306/11051010026.

Pezard, P. A., and S. M. Luthi (1988), Borehole electrical images in the basement of the Cajon Pass Scientific Drillhole, California; Fracture identification and tectonic implications, Geophysical Research Letters, 15(9), 1017-1020, doi: 10.1029/GL015i009p01017.

Pfeifer, N., and C. Briese (2007), Laser scanning - principles and applications, 3rd International Exhibition $\mathscr{G}$ Scientific Congress on Geodesy, Mapping, Geology, Geophysics, Novosibirsk, Russia.

Phillips, O. M. (1991), Flow and reactions in permeable rocks, Cambridge University Press.

Pickering, G., J. Bull, and D. Sanderson (1995), Sampling power-law distributions, Tectonophysics, 248(1-2), 1-20, doi:10.1016/0040-1951(95)00030-q.

Place, J., J. Sausse, J.-M. Marthelot, M. Diraison, Y. Géraud, and C. Naville (2011), 3$\mathrm{D}$ mapping of permeable structures affecting a deep granite basement using isotropic 3C VSP data, Geophysical Journal International, 186(1), 245-263, doi:10.1111/j. 1365-246x.2011.05012.x.

Pollard, D., and A. Aydin (1988), Progress in understanding jointing over the past century, Geological Society of America Bulletin, 100(8), 1181-1204, doi:10.1130/ 0016-7606(1988)100<1181:PIUJOT $\rangle 2.3 . \mathrm{CO} ; 2$.

Pollyea, R. M., and J. P. Fairley (2011), Estimating surface roughness of terrestrial laser scan data using orthogonal distance regression, Geology, 39(7), 623-626, doi: 10.1130/g32078.1.

Pollyea, R. M., and J. P. Fairley (2012), Implications of spatial reservoir uncertainty for $\mathrm{CO}_{2}$ sequestration in the east Snake River Plain, Idaho (USA), Hydrogeology Journal, 20(4), 689-699, doi:10.1007/s10040-012-0847-1.

Pollyea, R. M., J. P. Fairley, R. K. Podgorney, and T. L. Mcling (2014), Physical constraints on geologic $\mathrm{CO}_{2}$ sequestration in low-volume basalt formations, Geological Society of America Bulletin, 126(3-4), 344-351, doi:10.1130/b30874.1. 
Poppelreiter, M., C. Garcia-Carballido, and M. Kraaijveld (Eds.) (2010), Dipmeter and Borehole Image Log Technology: AAPG Memoir 1992, 1st ed., 357 pp., AAPG.

Price, R. C., J. A. Gamble, I. E. M. Smith, R. Maas, T. Waight, R. B. Stewart, and J. Woodhead (2012), The Anatomy of an Andesite Volcano: a Time-Stratigraphic Study of Andesite Petrogenesis and Crustal Evolution at Ruapehu Volcano, New Zealand, Journal of Petrology, 53(10), 2139-2189, doi:10.1093/petrology/egs050.

Priest, S., and J. Hudson (1981), Estimation of discontinuity spacing and trace length using scanline surveys, International Journal of Rock Mechanics and Mining Sciences Ef Geomechanics Abstracts, 18(3), 183-197, doi:10.1016/0148-9062(81)90973-6.

Priest, S. D. (1993), Discontinuity Analysis for Rock Engineering, Springer Netherlands, doi:10.1007/978-94-011-1498-1.

Priest, S. D. (2004), Determination of Discontinuity Size Distributions from Scanline Data, Rock Mechanics and Rock Engineering, 37(5), 347-368, doi:10.1007/s00603004-0035-2.

Pruess, K. (1992), Brief guide to the MINC-Method for modeling flow and transport in fractured media, Tech. rep.

R Core Team (2015), R: A Language and Environment for Statistical Computing, R Foundation for Statistical Computing, Vienna, Austria.

Radilla, G., J. Sausse, B. Sanjuan, and M. Fourar (2012), Interpreting tracer tests in the enhanced geothermal system (EGS) of Soultz-sous-Forêts using the equivalent stratified medium approach, Geothermics, 44, 43-51, doi:10.1016/j.geothermics.2012. 07.001 .

Rae, A. J. (2007), Rotokawa Geology and Geophysics, Tech. rep., GNS Science Consultancy Report 2007/83.

Rae, A. J., A. McCoy-West, L. Ramirez, and D. D. McNamara (2010), Geology of Production Wells RK30L1 and RK30L2, Rotokawa Geothermal Field, Tech. rep., GNS Science Consultancy Report 2010/02.

Ramirez, L. E., and A. J. Rae (2009), Geology of Injection Well RK24/RK24 ST1 Rotokawa Geothermal Field, Tech. rep., GNS Science Consultancy Report 2009/90.

Remaud, L. (2015), Study of the damage zone around the Alpine Fault, New-Zealand, from borehole data of the Deep Fault Drilling Project (stage 2), Msc, Université Joseph Fourier, Grenoble, France.

Renshaw, C., and J. Park (1997), Effect of mechanical interactions on the scaling of fracture length and aperture, Nature, 386, 482-484, doi:10.1038/386482a0. 
Reyes, A., B. Christenson, and K. Faure (2010), Sources of solutes and heat in low-enthalpy mineral waters and their relation to tectonic setting, New Zealand, Journal of Volcanology and Geothermal Research, 192(3-4), 117-141, doi:10.1016/j. jvolgeores.2010.02.015.

Reyners, M., D. Eberhart-Phillips, G. Stuart, and Y. Nishimura (2006), Imaging subduction from the trench to $300 \mathrm{~km}$ depth beneath the central North Island, New Zealand, with Vp and Vp/Vs, Geophysical Journal International, 165(2), 565-583, doi:10.1111/j.1365-246x.2006.02897.x.

Reynolds, A. P., G. Richards, B. de la Iglesia, and V. J. Rayward-Smith (2006), Clustering Rules: A Comparison of Partitioning and Hierarchical Clustering Algorithms, Journal of Mathematical Modelling and Algorithms, 5(4), 475-504, doi: 10.1007/s10852-005-9022-1.

Rider, M. (1996), The geological interpretation of well logs - Second Edition, 280 pp., Whittles Publishing.

Riquelme, A. J., A. Abellán, R. Tomás, and M. Jaboyedoff (2014), A new approach for semi-automatic rock mass joints recognition from 3D point clouds, Computers 8 Geosciences, 68, 38-52, doi:10.1016/j.cageo.2014.03.014.

Risk, G. F. (2000), Electrical resistivity surveys of the Rotokawa Geothermal Field, New Zealand, Proceedings of the 22nd New Zealand Geothermal Workshop, pp. 121126.

Rives, T., M. Razack, J.-P. Petit, and K. Rawnsley (1992), Joint spacing: analogue and numerical simulations, Journal of Structural Geology, 14(8-9), 925-937, doi: 10.1016/0191-8141(92)90024-q.

Rotevatn, A., S. J. Buckley, J. A. Howell, and H. Fossen (2009), Overlapping faults and their effect on fluid flow in different reservoir types: A LIDAR-based outcrop modeling and flow simulation study, AAPG Bulletin, 93(3), 407-427, doi:10.1306/ 09300807092.

Rowland, J. V., and R. H. Sibson (2001), Extensional fault kinematics within the Taupo Volcanic Zone, New Zealand: Soft-linked segmentation of a continental rift system, New Zealand Journal of Geology and Geophysics, 44(2), 271-283, doi:10. 1080/00288306.2001.9514938.

Rowland, J. V., and R. H. Sibson (2004), Structural controls on hydrothermal flow in a segmented rift system, Taupo Volcanic Zone, New Zealand, Geofluids, 4(4), 259-283, doi:10.1111/j.1468-8123.2004.00091.x. 
Rowland, J. V., and S. F. Simmons (2012), Hydrologic, Magmatic, and Tectonic Controls on Hydrothermal Flow, Taupo Volcanic Zone, New Zealand: Implications for the Formation of Epithermal Vein Deposits, Economic Geology, 107(3), 427-457, doi:10.2113/econgeo.107.3.427.

Rowland, J. V., C. J. Wilson, and D. M. Gravley (2010), Spatial and temporal variations in magma-assisted rifting, Taupo Volcanic Zone, New Zealand, Journal of Volcanology and Geothermal Research, 190(1-2), 89-108, doi:10.1016/j.jvolgeores. 2009.05.004.

Ruiu, J., G. Caumon, and S. Viseur (2015), Semiautomatic interpretation of 3D sedimentological structures on geologic images: An object-based approach, Interpretation, 3(3), SX63-SX74, doi:10.1190/int-2015-0004.1.

Sari, M., C. Karpuz, and C. Ayday (2010), Estimating rock mass properties using Monte Carlo simulation: Ankara andesites, Computers \& Geosciences, 36(7), 959969, doi:10.1016/j.cageo.2010.02.001.

Sasaki, M. (2006), Statistical Features of Vein Systems in the Hishikari Epithermal Gold Deposit, Japan, Resource Geology, 56(1), 27-36, doi:10.1111/j.1751-3928.2006. tb00265.x.

Schaefer, C. J., and S. A. Kattenhorn (2004), Characterization and evolution of fractures in low-volume pahoehoe lava flows, eastern Snake River Plain, Idaho, Geological Society of America Bulletin, 116(3-4), 322-336, doi:10.1130/b25335.1.

Schleicher, A. M., R. Sutherland, J. Townend, V. G. Toy, and B. van der Pluijm (2015), Clay mineral formation and fabric development in the DFDP-1B borehole, central Alpine Fault, New Zealand, New Zealand Journal of Geology and Geophysics, 58(1), 13-21, doi:10.1080/00288306.2014.979841.

Schnabel, R., R. Wahl, and R. Klein (2007), Efficient RANSAC for Point-Cloud Shape Detection, Computer Graphics Forum, 26(2), 214-226, doi:10.1111/j.14678659.2007.01016.x.

Schöpfer, M. P., A. Arslan, J. J. Walsh, and C. Childs (2011), Reconciliation of contrasting theories for fracture spacing in layered rocks, Journal of Structural Geology, $33(4), 551-565$, doi:10.1016/j.jsg.2011.01.008.

Schwarz, G. (1978), Estimating the dimension of a model, The Annals of Statistics, 6(2), 461-464, doi:10.1214/aos/1176344136.

Seebeck, H., A. Nicol, T. Stern, H. Bibby, and V. Stagpoole (2010), Fault controls on the geometry and location of the Okataina Caldera, Taupo Volcanic Zone, New 
Zealand, Journal of Volcanology and Geothermal Research, 190(1-2), 136-151, doi: 10.1016/j.jvolgeores.2009.04.011.

Seebeck, H., A. Nicol, P. Villamor, J. Ristau, and J. Pettinga (2014a), Structure and kinematics of the Taupo Rift, New Zealand, Tectonics, 33(6), 1178-1199, doi:10. 1002/2014tc003569.

Seebeck, H., A. Nicol, J. Walsh, C. Childs, R. Beetham, and J. Pettinga (2014b), Fluid flow in fault zones from an active rift, Journal of Structural Geology, 62, 52-64, doi:10.1016/j.jsg.2014.01.008.

Sewell, S., W. Cumming, L. Azwar, and C. Bardsley (2012), Integrated MT and natural state temperature interpretation for a conceptual model supporting reservoir numerical modelling and well targeting at the Rotokawa Geothermal Field, New Zealand, Proceedings of the Thirty-Seventh Workshop on Geothermal Reservoir Engineering.

Shanley, R. J., and M. A. Mahtab (1976), Delineation and analysis of clusters in orientation data, Journal of the International Association for Mathematical Geology, 8(1), 9-23, doi:10.1007/bf01039681.

Sherburn, S., S. Sewell, S. Bourguignon, W. Cumming, S. Bannister, C. Bardsley, J. Winick, J. Quinao, and I. Wallis (2015), Microseismicity at Rotokawa geothermal field, New Zealand, 2008-2012, Geothermics, 54, 23-34, doi:10.1016/j.geothermics. 2014.11.001.

Sibson, R. (1996), Structural permeability of fluid-driven fault-fracture meshes, Journal of Structural Geology, 18(8), 1031-1042, doi:10.1016/0191-8141(96)00032-6.

Sibson, R., S. White, and B. Atkinson (1981), Structure and distribution of fault rocks in the Alpine Fault Zone, New Zealand, Geological Society, London, Special Publication, 9, 197-210, doi:10.1144/GSL.SP.1981.009.01.18.

Sibson, R. H. (1992), Implications of fault-valve behaviour for rupture nucleation and recurrence, Tectonophysics, 211(1-4), 283-293, doi:10.1016/0040-1951(92)90065-E.

Siler, D. L., J. E. Faulds, B. Mayhew, and D. D. McNamara (2016), Analysis of the favorability for geothermal fluid flow in 3D: Astor Pass geothermal prospect, Great Basin, northwestern Nevada, USA, Geothermics, 60, 1-12, doi:10.1016/j. geothermics.2015.11.002.

Simmons, S. F., K. L. Brown, P. R. Browne, and J. V. Rowland (2016), Gold and silver resources in Taupo Volcanic Zone geothermal systems, Geothermics, 59, 205214, doi:10.1016/j.geothermics.2015.07.009. 
Simpson, M. P., S. S. Palinkas, M. Nieuwoudt, R. J. Bodnar, and J. L. Mauk (2015), Geothermal systems and epithermal deposits of New Zealand: A fluid chemistry comparison, Proceedings of the 37th New Zealand Geothermal Workshop.

Sirat, M., and C. J. Talbot (2001), Application of artificial neural networks to fracture analysis at the Äspö HRL, Sweden: Fracture sets classification, International Journal of Rock Mechanics and Mining Sciences, 38(5), 621-639, doi:10.1016/S13651609(01)00030-2.

Siratovich, P. A., M. J. Heap, M. C. Villeneuve, J. W. Cole, and T. Reuschlé (2014), Physical property relationships of the Rotokawa Andesite, a significant geothermal reservoir rock in the Taupo Volcanic Zone, New Zealand, Geothermal Energy, 2(10), doi:10.1186/s40517-014-0010-4.

Siratovich, P. A., F. von Aulock, Y. Lavallée, J. Cole, B. Kennedy, and M. Villeneuve (2015), Thermoelastic properties of the Rotokawa Andesite: A geothermal reservoir constraint, Journal of Volcanology and Geothermal Research, 301, 1-13, doi:10.1016/ j.jvolgeores.2015.05.003.

Siratovich, P. A., M. J. Heap, M. C. Villeneuve, J. W. Cole, B. M. Kennedy, J. Davidson, and T. Reuschlé (2016), Mechanical behaviour of the Rotokawa Andesites (New Zealand): Insight into permeability evolution and stress-induced behaviour in an actively utilised geothermal reservoir, Geothermics, 64, 163-179, doi: 10.1016/j.geothermics.2016.05.005.

Slatcher, N., M. James, S. Calvari, G. Ganci, and J. Browning (2015), Quantifying Effusion Rates at Active Volcanoes through Integrated Time-Lapse Laser Scanning and Photography, Remote Sensing, 7(11), 14,967-14,987, doi:10.3390/rs71114967.

Sonney, R., and B. Mountain (2013), Experimental simulation of greywacke-fluid interaction under geothermal conditions, Geothermics, 47, 27-39, doi:10.1016/j. geothermics.2012.11.003.

Spörli, K., and J. Rowland (2006), 'Column on column' structures as indicators of lava/ice interaction, Ruapehu andesite volcano, New Zealand, Journal of Volcanology and Geothermal Research, 157(4), 294-310, doi:10.1016/j.jvolgeores.2006.04.004.

Spörli, K. B., and H. Cargill (2011), Structural Evolution of a World-Class Epithermal Orebody: The Martha Hill Deposit, Waihi, New Zealand, Economic Geology, 106 (6), 975-998, doi:10.2113/econgeo.106.6.975.

Stasinopoulos, D. M., and R. A. Rigby (2007), Generalized Additive Models for Location Scale and Shape (GAMLSS) in R, Journal of Statistical Software, 23(7), 1-46, doi:10.18637/jss.v023.i07. 
Stern, T., D. Okaya, S. Kleffmann, M. Scherwath, S. Henrys, and F. Davey (2007), Geophysical exploration and dynamics of the Alpine Fault Zone, in A Continental Plate Boundary: Tectonics at South Island, New Zealand, edited by D. Okaya, T. Stern, and F. Davey, pp. 207-233, American Geophysical Union, Washington, D. C., doi:10.1029/175gm11.

Sturzenegger, M., and D. Stead (2009a), Close-range terrestrial digital photogrammetry and terrestrial laser scanning for discontinuity characterization on rock cuts, Engineering Geology, 106(3-4), 163-182, doi:10.1016/j.enggeo.2009.03.004.

Sturzenegger, M., and D. Stead (2009b), Quantifying discontinuity orientation and persistence on high mountain rock slopes and large landslides using terrestrial remote sensing techniques, Natural Hazards and Earth System Sciences, 9(2), 267-287, doi: 10.5194/nhess-9-267-2009.

Sutherland, R., D. Eberhart-Phillips, R. A. Harris, T. Stern, J. Beavan, S. Ellis, S. Henrys, S. Cox, R. J. Norris, K. R. Berryman, J. Townend, S. Bannister, J. Pettinga, B. Leitner, L. Wallace, T. A. Little, A. F. Cooper, M. Yetton, and M. Stirling (2007), Do Great Earthquakes Occur on the Alpine Fault in Central South Island, New Zealand?, in A Continental Plate Boundary: Tectonics at South Island, New Zealand, edited by D. Okaya, T. Stern, and F. Davey, pp. 235-251, American Geophysical Union, Washington, D. C., doi:10.1029/175GM12.

Sutherland, R., V. G. Toy, J. Townend, S. C. Cox, J. D. Eccles, D. R. Faulkner, D. J. Prior, R. J. Norris, E. Mariani, C. Boulton, B. M. Carpenter, C. D. Menzies, T. A. Little, M. Hasting, G. P. D. Pascale, R. M. Langridge, H. R. Scott, Z. R. Lindroos, B. Fleming, and A. J. Kopf (2012), Drilling reveals fluid control on architecture and rupture of the Alpine fault, New Zealand, Geology, 40(12), 1143-1146, doi: 10.1130/g33614.1.

Sutherland, R., J. Townend, V. G. Toy, M. Allen, L. Baratin, N. Barth, Beacroft., A. Benson, C. Boese, A. Boles, C. Boulton, L. Capova, B. M. Carpenter, B. Célérier, C. Chamberlain, R. Conze, A. Cooper, J. Coussens, A. Coutts, S. Cox, L. Craw, M. Doan, J. Eccles, D. R. Faulkner, A. Grieve, J. Grochowski, A. Gulley, G. Henry, J. Howarth, K. Jacobs, T. Jeppson, N. Kato, S. Keys, M. Kirilova, Y. Kometani, A. Kovacs, R. M. Langridge, W. Lin, T. Little, D. Mallyon, B. Mariani, R. Marx, C. Massiot, L. Mathewson, B. Melosh, C. Menzies, J. Moore, L. Morales, C. Morgan, H. Mori, A. Niemeijera, O. Nishikawa, O. Nitsch, J. Paris Cavailhès, B. Pooley, D. J. Prior, A. Pyne, K. Sauer, M. Savage, A. Schleicher, D. R. Schmitt, N. Shigematsu, S. Taylor-Offord, H. Tobin, P. Upton, R. Valdez, K. Weaver, T. Wiersberg, W. J., S. Yeo, and M. Zimmer (2015), Deep Fault Driling Project (DFDP), Alpine Fault 
Boreholes DFDP-2A and DFDP-2B Technical Completion Report, Tech. rep., GNS Science Report 2015/50.

Sutherland, R., J. Townend, V. G. Toy, P. Upton, and the DFDP-2B Science Team (submitted), Extreme hydrothermal conditions near an active plate-bounding fault, Nature.

Terzaghi, R. D. (1965), Sources of Error in Joint Surveys, Géotechnique, 15(3), 287304, doi:10.1680/geot.1965.15.3.287.

Tokhmechi, B., H. Memarian, B. Moshiri, V. Rasouli, and H. A. Noubari (2011), Investigating the validity of conventional joint set clustering methods, Engineering Geology, 118(3-4), 75-81, doi:10.1016/j.enggeo.2011.01.002.

Torabi, A., and S. S. Berg (2011), Scaling of fault attributes: A review, Marine and Petroleum Geology, 28(8), 1444-1460, doi:10.1016/j.marpetgeo.2011.04.003.

Tost, M., and S. J. Cronin (2015), Linking distal volcaniclastic sedimentation and stratigraphy with the development of Ruapehu volcano, New Zealand, Bulletin of Volcanology, 77 (11), doi:10.1007/s00445-015-0977-0.

Townend, J., and M. D. Zoback (2000), How faulting keeps the crust strong, Geology, 28 (5), 399-402, doi:10.1130/0091-7613(2000)28〈399:HFKTCS $\rangle 2.0 . C O ; 2$.

Townend, J., R. Sutherland, and V. Toy (2009), Deep Fault Drilling Project - Alpine Fault, New Zealand, Scientific Drilling, 8, doi:10.2204/iodp.sd.8.12.2009.

Townend, J., R. Sutherland, V. G. Toy, J. D. Eccles, C. Boulton, S. C. Cox, and D. McNamara (2013), Late-interseismic state of a continental plate-bounding fault: Petrophysical results from DFDP-1 wireline logging and core analysis, Alpine Fault, New Zealand, Geochemistry, Geophysics, Geosystems, 14(9), 3801-3820, doi: 10.1002/ggge.20236.

Toy, V. G., D. J. Prior, R. J. Norris, A. F. Cooper, and M. Walrond (2012), Relationships between kinematic indicators and strain during syn-deformational exhumation of an oblique slip, transpressive, plate boundary shear zone: The Alpine Fault, New Zealand, Earth and Planetary Science Letters, 333-334, 282-292, doi: 10.1016/j.epsl.2012.04.037.

Toy, V. G., R. Norris, D. Prior, M. Walrond, and A. Cooper (2013), How do lineations reflect the strain history of transpressive shear zones? The example of the active Alpine Fault zone, New Zealand, Journal of Structural Geology, 50, 187-198, doi: 10.1016/j.jsg.2012.06.006. 
Toy, V. G., C. J. Boulton, R. Sutherland, J. Townend, R. J. Norris, T. A. Little, D. J. Prior, E. Mariani, D. Faulkner, C. D. Menzies, H. Scott, and B. M. Carpenter (2015), Fault rock lithologies and architecture of the central Alpine fault, New Zealand, revealed by DFDP-1 drilling, Lithosphere, 7(2), 155-173, doi:10.1130/1395.1.

Ulusay, R., and J. A. Hudson (Eds.) (2007), The complete ISRM suggested methods for rock characterization, testing and monitoring: 1974-2006, International Soc. for Rock Mechanics, Commission on Testing Methods.

Umili, G., A. Ferrero, and H. Einstein (2013), A new method for automatic discontinuity traces sampling on rock mass 3D model, Computers $\&$ Geosciences, 51, 182-192, doi:10.1016/j.cageo.2012.07.026.

Upton, P., and D. Craw (2014), Modelling of structural and lithological controls on mobility of fluids and gold in orogenic belts, New Zealand, Geological Society, London, Special Publications, 402(1), 231-253, doi:10.1144/sp402.1.

U.S. Energy Information Administration (2014), Annual Energy Outlook 2014, Tech. rep., doi:10.1007/s13398-014-0173-7.2.

Vermilye, J. M., and C. H. Scholz (1995), Relation between vein length and aperture, Journal of Structural Geology, 17(3), 423-434, doi:10.1016/0191-8141(94)00058-8.

Villaescusa, E., and E. T. Brown (1992), Maximum Likelihood Estimation of Joint Size from Trace Length Measurements, Rock Mechanics and Rock Engineering, 25(2), 6787, doi:10.1007/BF01040513.

Villamor, P., and K. R. Berryman (2001), A Late Quaternary extension rate in the Taupo Volcanic Zone, New Zealand, derived from fault slip data, New Zealand Journal of Geology and Geophysics, 44(2), 243-269.

Villamor, P., and K. R. Berryman (2006), Evolution of the southern termination of the Taupo Rift, New Zealand, New Zealand Journal of Geology and Geophysics, 49(1), 23-37, doi:10.1080/00288306.2006.9515145.

Viseur, S. (2004), Turbidite reservoir characterization: Object-based stochastic simulation meandering channels, Bulletin de la Societé Géologique de France, 175(1), 11-20, doi:10.2113/175.1.11.

Voight, B. (2000), Structural stability of andesite volcanoes and lava domes, Philosophical Transactions of the Royal Society A: Mathematical, Physical and Engineering Sciences, 358(1770), 1663-1703, doi:10.1098/rsta.2000.0609. 
Vollmer, F. W. (1995), C program for automatic contouring of spherical orientation data using a modified Kamb method, Computers and Geosciences, 21(1), 31-49, doi:10.1016/0098-3004(94)00058-3.

Vye-Brown, C., S. Self, and T. L. Barry (2013), Architecture and emplacement of flood basalt flow fields: case studies from the Columbia River Basalt Group, NW USA, Bulletin of Volcanology, 75 (3), doi:10.1007/s00445-013-0697-2.

Wagner, W., and H. Kretzschmar (2007), International Steam Tables - Properties of Water and Steam based on the Industrial Formulation IAPWS-IF97, 2nd ed., 350 pp., Springer Science \& Business Media, doi:10.1007/978-3-540-74234-0.

Wallace, L. M., J. Beavan, R. McCaffrey, and D. Darby (2004), Subduction zone coupling and tectonic block rotations in the North Island, New Zealand, Journal of Geophysical Research: Solid Earth, 109(B12), doi:10.1029/2004jb003241.

Wallis, I. C., D. McNamara, J. V. Rowland, and C. Massiot (2012a), The nature of fracture permeability in the basement greywacke at Kawerau Geothermal Field, New Zealand, Proceedings of the 37th Workshop on Geothermal Reservoir Engineering.

Wallis, I. C., S. McCormick, S. Sewell, and C. Boseley (2012b), Formation Assessment in Geothermal Using Wireline Tools Application and Early Results from the Ngatamariki Geothermal Field, New Zealand, Proceedings of the New Zealand Geothermal Workshop.

Wallis, I. C., C. Bardsley, T. Powell, J. V. Rowland, and J. M. O'Brien (2013), A structural model for the Rotokawa Geothermal Field, New Zealand, Proceedings of the New Zealand Geothermal Workshop.

Wang, X. (2005), Stereological interpretation of rock fracture traces on borehole walls and other cylindrical surfaces, Ph.d., Virginia Polytechnic Institute and State University.

Wightman, R. H., and T. A. Little (2007), Deformation of the Pacific Plate above the Alpine fault ramp and its relationship to expulsion of metamorphic fluids: An array of backshears, in A Continental Plate Boundary: Tectonics at South Island, New Zealand, edited by D. Okaya, T. Stern, and F. Davey, pp. 177-205, Wiley-Blackwell, doi:10.1029/175gm10.

Williams, J., V. G. Toy, C. Massiot, D. D. McNamara, and T. Wang (2015), Quantitative Characterisation of Fracturing Around the Damage Zone Surrounding New Zealand's Alpine Fault Using X-ray CT Scans of DFDP-1 Core, AGU Fall meeting. 
Williams, J., V. G. Toy, C. Massiot, D. D. McNamara, and T. Wang (2016), Damaged beyond repair? Characterising the damage zone of a fault late in its interseismic cycle, the Alpine Fault, New Zealand, Journal of Structural Geology, 90, 76-94, doi:10.1016/j.jsg.2016.07.006.

Williams, J. H., and C. D. Johnson (2004), Acoustic and optical borehole-wall imaging for fractured-rock aquifer studies, Journal of Applied Geophysics, 55(1-2), 151-159, doi:10.1016/j.jappgeo.2003.06.009.

Wilson, C., B. Houghton, M. McWilliams, M. Lanphere, S. Weaver, and R. Briggs (1995), Volcanic and structural evolution of Taupo Volcanic Zone, New Zealand: a review, Journal of Volcanology and Geothermal Research, 68(1-3), 1-28, doi:10. 1016/0377-0273(95)00006-g.

Wilson, C. E., A. Aydin, M. Karimi-Fard, L. J. Durlofsky, A. Sagy, E. E. Brodsky, O. Kreylos, and L. H. Kellogg (2011), From outcrop to flow simulation: Constructing discrete fracture models from a LIDAR survey, AAPG Bulletin, 95(11), 1883-1905, doi:10.1306/03241108148.

Wilson, C. J., and B. L. Charlier (2016), The life and times of silicic volcanic systems, Elements, 12(2), 103-108, doi:10.2113/gselements.12.2.103.

Wilson, C. J. N., and J. V. Rowland (2016), The volcanic, magmatic and tectonic setting of the Taupo Volcanic Zone, New Zealand, reviewed from a geothermal perspective, Geothermics, 59(B), 168-187, doi:10.1016/j.geothermics.2015.06.013.

Wyering, L. D., M. C. Villeneuve, I. C. Wallis, P. A. Siratovich, B. M. Kennedy, D. Gravley, and J. L. Cant (2014), Mechanical and physical properties of hydrothermally altered rocks, Taupo Volcanic Zone, New Zealand, Journal of Volcanology and Geothermal Research, 288, 76-93, doi:10.1016/j.jvolgeores.2014.10.008.

Wysoczanski, R. (1989), Volcanology and pretology of the Iwikau member, Whakapapa Formation, Mt Ruapehu, Tongariro Volcanic Centre, New Zealand, B.sc. (Hns), Victoria University of Wellington, New Zealand.

Yow, J. (1987), Blind zones in the acquisition of discontinuity orientation data, International Journal of Rock Mechanics and Mining Sciences $\&$ Geomechanics Abstracts, 24(5), 317-318, doi:10.1016/0148-9062(87)90868-0.

Zadeh, L. (1965), Fuzzy sets, Information and Control, 8(3), 338-353, doi:10.1016/ s0019-9958(65)90241-x.

Zemanek, J., E. E. Glenn, L. J. Norton, and R. L. Caldwell (1970), Formation evaluation by inspection with the borehole televiewer, Geophysics, 35 (2), 254-269, doi: 10.1190/1.1440089. 
Zhang, L., H. H. Einstein, and W. S. Dershowitz (2002), Stereological relationship between trace length and size distribution of elliptical discontinuities, Géotechnique, 52(6), 419-433, doi:10.1680/geot.2002.52.6.419.

Zhou, W., and N. H. Maerz (2002), Implementation of multivariate clustering methods for characterizing discontinuities data from scanlines and oriented boreholes, Computers \& Geosciences, 28(7), 827-839, doi:10.1016/s0098-3004(01)00111-x.

Zoback, M., C. Barton, M. Brudy, D. Castillo, T. Finkbeiner, B. Grollimund, D. Moos, P. Peska, C. Ward, and D. Wiprut (2003), Determination of stress orientation and magnitude in deep wells, International Journal of Rock Mechanics and Mining Sciences, 40(7-8), 1049-1076, doi:10.1016/j.ijrmms.2003.07.001.

Zoback, M., S. Hickman, and W. Ellsworth (2007), The Role of Fault Zone Drilling, in Treatise on Geophysics, edited by G. Schubert, pp. 649-674, Elsevier BV, doi: 10.1016/b978-044452748-6.00084-5.

Zoback, M. D. (2007), Reservoir geomechanics, 449 pp., Cambridge University Press, doi:10.1017/CBO9780511586477.

Zuquim, M., and J. V. Rowland (2013), Structural controls on fluid flow at the Onemana area, Coromandel Peninsula, New Zealand, Proceedings of the 35th NZ Geothermal Workshop. 